\title{
CAN WE IMPROVE MYOCARDIAL PROTECTION DURING ISCHEMIC INJURY?
}

\author{
R. Ferrari \\ Department of Cardiology and LTTA Centre, University Hospital of Ferrara, \\ Ferrara and Maria Cecilia Hospital, GVM Care \& Research, E.S. Health Science Foundation, \\ Cotignola, Italy
}

Cardiac protection is a broad term that refers to all strategies aimed at the attenuation of the damage caused by myocardial ischaemia (MI) and reperfusion. The term is used both in the experimental and clinical field, embracing a very wide (too wide in fact!) range of conditions from reduction of coronary atherosclerosis progression to an immediate reduction of the myocardial infarct size due to acute ischaemia and/or reperfusion. There is no doubt that cardiac protection is a story of broad success but there are also some failures. Indeed, the progression of coronary atherosclerosis can be reduced with angiotensin II inhibitors and statins with a consequential improvement of outcome. Equally, after the demonstration that coronary thrombosis is the cause and not the result of MI, timely restoration of blood flow (reperfusion) has become the standard treatment for these patients, with a corresponding limitation of infarct size, long-term improvement of MI and, more importantly, a reduction in mortality. Despite this success, however, the process of late restoration of blood flow to the ischaemic myocardium can paradoxically induce injury, a phenomenon known as reperfusion injury (RI). Therefore, the major challenge of cardiac protection today is to reduce RI. The problem is that $\mathrm{RI}$ is a complex phenomenon, involving many unclearly defined players, all contributing to the final damage that is inflicted on the heart. RI was originally described as a cell swelling, hyper-contracture or disruption of the intra-cellular structure and, in time, has progressed to at least four other types of cardiac dysfunction such as: myocardial stunning, the no-reflow phenomenon, reperfusion arrhythmias and finally lethal reperfusion injury described as an independent mediator of cardiomyocyte death (either necrosis, apoptosis, autophagy or necroptosis), different from ischaemic injury. Several approaches to reduce RI were tried with antioxidants, aderosine, beta and CA2+ blockers, statins, glucose with or without insulin and $\mathrm{K}$, Trimetazidine, cyclosporine and other inhibitors of the $\mathrm{Ca2}$ + pore - all without success. Recently, non-pharmacological interventions have also been proposed to reduce $\mathrm{RI}$, mainly pre and post conditioning. The ideas come from the experimental laboratory, showing that brief cycles of ischaemia and reperfusion performed before a prolonged coronary occlusion or after reperfusion reduced infarct size in dogs. However, when applied to the clinic (mainly post-conditioning as pre-conditioning is difficult) protocols produced good results in small, proof of concept trials but not in a large randomised clinical trial.

No conflict of interest 


\title{
9
}

\section{INTERVENTIONAL CARDIOLOGY: TOPICAL ISSUES}

\section{CULPRIT-ONLY VERSUS COMPLETE REVASCULARIZATION STRATEGIES TO TREAT MULTIVESSEL DISEASE AFTER PRIMARY PCI FOR STEMI}

\author{
G. Di Pasquale, E. Filippini, P. Camillo Pavesi, G. Franco Tortorici, G. Casellai, P. Sangiorgio \\ Department of Cardiology, Maggiore Hospital, Bologna, Italy \\ In $30 \%$ to $60 \%$ of patients presenting with STEMI significant stenoses are present in one or more \\ non infarct-related arteries (IRA). The 2014 ESC/EACTS guidelines give a class Ilb (level B) \\ recommendation for immediate revascularization of non-culprit artery in selected patients.
}

Since the STEMI guidelines publication, three randomized clinical trials have shown a significant benefit of complete revascularization when compared with culprit-only PCI. In the PRAMI trial $(\mathrm{n}=$ 465) complete revascularization at the time of culprit artery PCI was associated with significant reduction in cardiovascular outcomes when compared with culprit-only PCI. Similarly, in the CVLPRIT trial $(n=296)$, complete revascularization at index hospitalization was associated with a significant reduction of composite primary endpoint compared with treating only the IRA. In the DANAMI 3 - PRIMULTI ( $n=627)$ FFR-guided complete revascularization before discharge significantly reduces the composite primary end-point of mortality, non-fatal re-infarction, and ischemia-driven revascularization compared with only culprit lesion $\mathrm{PCl}$.

Four meta-analyses recently published in 2014 and 2015, including randomized and nonrandomized clinical trials, have shown concordant results in terms of better outcome in favour of complete revascularization. However, there is firm evidence only for reduction of MACE with complete revascularization largely driven by reduction in repeat revascularization, without firm evidence for the reduction in death or myocardial infarction when compared with culprit-only revascularization.

In conclusion, the current available evidence from the randomized clinical trials, with a total sample size of a bit more than 1700 patients, is not robust enough to firmly recommend complete revascularization in STEMI patients. This uncertainty lend support to the continuation of the COMPLETE trial. This ongoing trial is anticipated to enroll 3900 patients with STEMI from across the world and will be powered for hard outcomes of death and myocardial infarction. Until the results of the COMPLETE trial are reported, physicians need to individualize care regarding the opportunity and the timing of the non-IRA PCI.

No conflict of interest 
10

INTERVENTIONAL CARDIOLOGY: TOPICAL ISSUES

SIGNIFICANCE OF INCOMPLETE REVASCULARIZATION IN STABLE AND UNSTABLE CAD

\section{S.J. Brener}

Cardiac Catheterization Laboratory, NY Methodist Hospital, New York, NY, USA

Aims: To describe the impact of complete revscularization, anatomical or functional, on clinical outcomes in patients with stable or unstable coronary artery disease undergoing percutaneous or surgical revascularization.

Method: Review of data from clinical trials and national registries spanning two decades.

Results: In the Medicine, Angioplasty or Surgery (MAAS II) study, 611 patients with multivessel coronary artery disease were randomized to one of the above three arms. In a retrospective analysis, complete revascularization was achieved in $64 \%$ of the CABG group and $36 \%$ of the $\mathrm{PCl}$ cohort. At 10 years, the rate of cardiovascular death in patients with complete

revascularization was significantly lower than in those with incomplete revascularization, $9.3 \%$ vs. $15.7 \%, P=0.04$.

In patients with non-ST-segment elevation myocardial infarction and multivessel coronary artery disease, more complete revascularization is also associated with better outcomes. In a metaanalysis of 8 trials encompassing over 8,000 patients, multivessel $\mathrm{PCl}$ was an independent predictor of lower rate of major adverse cardiac events - HR=0.74 (95\% CI 0.57 to 0.97$), \mathrm{P}<0.05$. In a similar analysis from 4 studies of ST-segment elevation myocardial infarction with nearly 1,000 subjects, there was a significant reduction in all-cause mortality - RR $=0.57,95 \%$ confidence interval $[\mathrm{Cl}] 0.36$ to $0.92, \mathrm{p}=0.02$ ) and in cardiac death (RR $0.38,95 \% \mathrm{Cl} 0.20$ to $0.73, p=0.004$ ) favoring more complete revscularization.

Conclusion: Thus, complete revascularization seems to provide superior outcomes compared with incomplete revscularization, particularly in patients with acute coronary syndromes and those with chronic total occlusions.

No conflict of interest 
13

INTERVENTIONAL CARDIOLOGY: TOPICAL ISSUES

IMPORTANCE OF DIRECT CORONARY IMAGING TO GUIDE OPTIMAL PCI

\author{
J.A. Goldstein \\ Department of Cardiovascular Medicine, Beaumont Health System, Royal Oak, MI USA
}

The ideal invasive coronary imaging tool should provide a complete roadmap of atherosclerotic burden throughout the coronary tree, delineate the architectural and compositional nature of each plaque, facilitate determination of coronary blood flow, and guide optimal PCI (delineation of optimal length of vessel stented, complete stent expansion and apposition, and detection of stent edge dissections and thrombosis). Unfortunately, coronary angiography underestimates the magnitude of atherosclerotic burden and has significant limitations guiding optimal PCl.

Employing angiography alone, stent complications (dissection, early or late stent thrombosis, or edge restenosis) may result from a geographical miss in stent placement (stent ends in an area with high plaque burden) or from incomplete stent expansion or malapposition. There is a growing body of evidence demonstrating that compared to angiographic guidance alone, $\mathrm{PCl}$ performed employing direct coronary imaging (IVUS, NIRS-IVUS, OCT) achieves superior outcomes by assuring complete lesion coverage, full stent expansion and apposition and to detect stent complications (e.g edge dissection and thrombosis). A recent meta-analysis (Zhang et al Eurointervention 2012;8:855-65) encompassing 11 studies in 19,619 documented the superiority of direct coronary imaging to facilitate optimal performance of $\mathrm{PCl}$; compared with angiographyguidance, IVUS-guided DES implantation was associated with a reduced incidence of Death (HR: $0.59,95 \% \mathrm{Cl}: 0.48-0.73$, p75\% cases, resulting in longer, appropriately sized stents. Most important, IVUS guidance improved clinical outcomes, with a 33\% reduction in $\mathrm{MI}, 50 \%$ reduction in stent thrombosis and 38\% reduction TVR. OCT achieves the highest spatial resolution of existing imaging modalities (10 microns vs 100 microns for IVUS) and is an excellent method to prove optimal stent expansion and apposition and detect edge dissections. In aggregate, these observations emphasize the value of performing $\mathrm{PCI}$ guided by direct coronary imaging.

No conflict of interest 
14

INTERVENTIONAL CARDIOLOGY: TOPICAL ISSUES

\section{VERY, VERY LATE DRUG-ELUTING STENT THROMBOSIS}

M.P. Savage

Cardiac Catheterization Laboratory, Thomas Jefferson University Hospital

Philadelphia, PA, USA

Aims: A serious long-term complication of drug-eluting stents (DES) is very late stent thrombosis (VLST) beyond 1 year after implantation. While VLST has been reported as late as 5 years following the procedure, it remains unknown whether DES thrombosis is a finite phenomenon that abates over time or is a risk that persists indefinitely. This study sought to describe a series of patients with "very", very late stent thrombosis (VVLST) defined as occurring more than 5 years after DES placement.

Method: A detailed review of medical records was performed to identify patients with ACS due to VVLST.

Results: The study group consisted of 8 patients (7 men, 1 woman), ages $32-70$ years, who had angiographically confirmed VVLST. Seven patients were active smokers and 4 were diabetic. The interval between stent implantation and VVLST ranged from 5.6 to 7.2 years. The DES was sirolimus-eluting in 5 and paclitaxel-eluting in 3 patients. None of the patients were taking clopidogrel and only 2 patients were taking aspirin at the time of VVLST. Therefore, 6 of the 8 patients were not on any antiplatelet therapy before VVLST. The clinical presentation was an acute $\mathrm{MI}$ in all patients (7 with STEMI). Seven patients were treated successfully by emergent repeat $\mathrm{PCl}$ and one patient postoperative from neurosurgery was managed medically.

Conclusion: The risk of stent thrombosis continues to persist well beyond 5 years after DES implantation. These sobering findings underscore the importance of long-term clinical vigilance and reinforce current $\mathrm{PCl}$ guidelines which recommend continuing at least aspirin indefinitely after DES.

No conflict of interest 
19

ACUTE MYOCARDIAL INFARCTION

DIAGNOSTIC AND THERAPEUTIC IMPLICATIONS OF TYPE 2 MYOCARDIAL INFARCTION

J.S. Alpert

Sarver Heart Center, University of Arizona College of Medicine, Tucson, AZ, USA

In 2007, the Task Force for the Universal Definition of Myocardial Infarction simultaneously published an international consensus document in the Journal of the American College of Cardiology, the European Heart Journal, and Circulation. The 2007 document was an updated revision of the original document from this group that had first been published in 2000. In this second communication, the task force defined 5 subtypes of myocardial infarction (MI) which were retained in the 2012 revision ${ }^{3}$.

Type 1 infarction is usually the result of atherosclerotic coronary artery disease (CAD) with thrombotic coronary arterial obstruction secondary to atherosclerotic plaque rupture or fissuring, although an occasional patient may demonstrate normal luminal coronary anatomy at catheterization despite the clinical syndrome of a type $1 \mathrm{Ml}$. In contrast patients with type $2 \mathrm{Ml}$ do not have atherosclerotic plaque rupture but myocardial necrosis because of an increase in myocardial oxygen demand and/or a decrease in myocardial blood flow. Type $3 \mathrm{Ml}$ is the result of coronary arterial thrombosis with early demise, and types 4 and $5 \mathrm{MI}$ are related to complications of percutaneous coronary intervention and coronary bypass surgery.

Type $2 \mathrm{MI}$ has been the subject of considerable clinical discussion and confusion. There has been continuing concern and debate concerning the criteria for diagnosing this latter entity and distinguishing it from type $1 \mathrm{Ml}$ and from myonecrosis secondary to a variety of entities other than myocardial ischemia.

\section{Type 1 vs. Type $2 \mathrm{MI}$}

Distinguishing patients with type $2 \mathrm{Ml}$ from those with type $1 \mathrm{Ml}$ is usually can at times be straightforward and at other times challenging. The clinical presentation is often definitive here. Patients with type $1 \mathrm{Ml}$ usually present with spontaneous symptoms such as chest discomfort with or without associated ischemic ECG changes and in the absence of a cause for increased myocardial oxygen demand, e.g., a marked tachycardia , or decreased myocardial blood flow, e.g., hypotension secondary to marked bradycardia. Patients with type $1 \mathrm{MI}$ often demonstrate suggestive ECG changes such as ST elevation or depression. During coronary angiography, type $1 \mathrm{Mls}$ are often found to have new or presumptive new coronary arterial occlusion and/or the presence of plaque rupture, fissure, or thrombus within a coronary artery.

\section{The Essence of a Type $2 \mathrm{MI}$}

In the most recent publications from the task force, type $2 \mathrm{MI}$ was categorized as a myocardial infarction secondary to an ischemic imbalance between blood supply and myocardial oxygen demand ${ }^{2,3}$. Patients may or may not have atherosclerotic coronary artery disease. 
Instances of myocardial injury with necrosis where an imbalance between myocardial oxygen supply and/or demand occur include coronary endothelial dysfunction, coronary artery spasm, coronary embolism, tachy-/brady-arrhythmias, anemia, respiratory failure, hypotension, and hypertension with or without left ventricular hypertrophy. New data concerning the differences between type 1 and type $2 \mathrm{MI}$ will be presented and discussed.

No conflict of interest 


\title{
ACUTE MYOCARDIAL INFARCTION
}

\section{REGIONAL SYSTEMS OF CARE FOR STEMI: THE US PERSPECTIVE}

\author{
H.L. Dauerman \\ University of Vermont Medical Center, Burlington, VT, USA
}

\begin{abstract}
Aims: Current ACC/AHA guidelines recommend transfer and primary PCI for STEMI patients within the time limit of first contact to device $\leq 120$ minutes. An overview of contemporary success with transfer for Primary PCl versus pharmaconvasive therapy in the U.S is warranted.
\end{abstract}

Method: Primary PCI transfer programs in the U.S and Europe are surveyed for feasability and guideline implementation success. For example, a nationwide database demonstrates the overall progress of primary $\mathrm{PCI}$ transfer in the U.S: 14,518 patients were transferred from non-PCI capable hospitals for primary PCI to 398 AHA Mission: Lifeline ${ }^{\circledR}$ hospitals between July 2008 and December 2012, with estimated transfer times $<60$ minutes.

Results: In this U.S national database, the median estimated transfer distance was 26.5 miles. First door to device $\leq 120$ minutes was achieved in $65 \%$ of patients $(\mathrm{N}=9,380)$. In addition to known predictors of delay (cardiogenic shock, cardiac arrest and prolonged door in door out time), STEMI referral hospitals' rural location and longer estimated transfer time were identified as predictors of delay. Lower PCI Hospital annual STEMI volume was a potent predictor of delay and these criteria may be used to delineate between pharmacoinvasive vs primary PCI regional transfer systems in the U.S.

Conclusion: More than one-third of U.S STEMI patients transferred for Primary PCI fail to achieve first door to device time $\leq 120$ minutes, despite estimated transfer times $<60$ minutes. Quality improvement initiatives include the Mission Lifeline STEMI Accelerator Program and regional commitments to pharmacoinvasive therapy, emergency department bypass and enhanced ambulance based transfer systems.

No conflict of interest 


\title{
21
}

\section{ACUTE MYOCARDIAL INFARCTION}

\section{IN-HOSPITAL STEMI - PREDICTORS, TREATMENT AND OUTCOMES}

\author{
R. Stouffer \\ UNC School of Medicine, Chappel Hill, North Carolina, USA
}

ST elevation myocardial infarction (STEMI) is most commonly caused by an acute thrombotic occlusion of a coronary artery. For patients who have the onset of STEMI outside of the hospital, early reperfusion therapy with either fibrinolysis or primary percutaneous coronary intervention $(\mathrm{PCl})$ reduces complications and improves survival. STEMI systems of care have been successful at reducing door to balloon times and mortality rates for STEMIs that with onset outside of the hospital (outpatient STEMI). In contrast, much less is known about STEMI that occurs in hospitalized patients. Recent studies have shown that in-hospital mortality of inpatient STEMI is $2-10$ fold higher than outpatient STEMI. Patients who develop STEMI while in the hospital are older, more often female, and have a higher prevalence of hypertension, diabetes with chronic complications, congestive heart failure, chronic kidney disease, known coronary artery disease, peripheral vascular disease, coagulopathy, metastatic cancer, and valvular diseases than outpatient STEMI. Inpatient STEMI are much less likely to undergo coronary angiography and/or PCI than outpatient STEMI. There are frequent system delays in diagnosis and infrequent use of reperfusion therapy for inpatient STEMI which likely contribute to unfavorable outcomes.

No conflict of interest 


\title{
ACUTE MYOCARDIAL INFARCTION
}

\section{NEW SIGHTS INTO THE PATHOPHYSIOLOGY OF ACUTE CORONARY SYNDROMES DERIVED FROM INTRAVASCULAR IMAGING}

\author{
R. Wilensky \\ Perelman School of Medicine, University of Pennsylvania, Philadephia, PA, USA
}

The traditional view of the pathophysiologic substrate of acute coronary syndromes was of nonhemodynamically significant lesions possessing thin fibrous caps in which cap rupture results in thrombus formation and an acute coronary syndrome. The thin cap fibroatheroma with an increased propensity towards rupture consists of a large necrotic core containing inflammatory cells and a thin fibrous cap, $<65 \mu \mathrm{m}$. Over the last decade the development and use of intravascular imaging has resulted in a more nuanced view of lesions causing a myocardial infarction, unstable angina or ischemic cardiac death, leading to additional questions concerning the natural history of "vulnerable plaques." On the basis of multiple invasive imaging studies it has been shown that lesions associated with future instability have a greater plaque burden, a higher remodeling index, and increased intramural lipid compared to stable lesions. While thinner fibrous caps are more common in lesions causing ACS than those in the setting of stable angina, several studies have demonstrated that cap thickness in the setting of ACS often exceeds $70 \mu \mathrm{m}$, occasionally as thick at $150 \mu \mathrm{m}$. Also, plaque erosion and subsequent thrombus formation causes ACS in over $1 / 3$ of patients and calcified nodules in an additional $10 \%$. There has been conflicting data regarding the changes in plaque morphology over time.

No conflict of interest 
ACUTE MYOCARDIAL INFARCTION

\title{
HEART RATE SLOWING WITH IVABRADINE IN PATIENTS WITH HEART FAILURE WHO ALSO HAVE ANGINA PECTORIS
}

\author{
J.S. Borer, K. Swedberg, M. Komajda, M. Boehm, L. Tavazzi, L. Ford \\ State University of New York Downstate Medical Center, New York, NY, USA
}

Background: Resting heart rate $(\mathrm{HR})$ is a modifiable risk factor for adverse outcomes in chronic systolic heart failure (CHF). Ivabradine reduces HR by selectively inhibiting the sinoatrial node $f-$ current (If). In the SHIFT study, HR reduction with ivabradine significantly reduced the composite endpoint of cardiovascular (CV) death or first hospitalization for worsening HF (WHF) in patients with CHF (LV ejection fraction [EF] $\leq 35 \%$ ) and in sinus rhythm with HR $\geq 70 \mathrm{bpm}$. In the SIGNIFY trial,HR lowering did not reduce CV death or nonfatal myocardial infarction (MI) in patients with coronary artery disease without LV dysfunction or clinical HF and slightly increased these events in the subgroup with angina. To assess the efficacy of ivabradine in CHF patients who have angina, we analyzed data for this subgroup in SHIFT.

Methods: Of the 6505 evaluable SHIFT patients, $68 \%$ had ischemic etiology; 2220 (34\%) had angina (ANG) at baseline. The effect of ivabradine was determined on the incidence of CV death or first WHF hospitalization, and on CV death or nonfatal MI, both in all SHIFT patients and in the ANG subgroup.

Results: Ivabradine reduced CV death or hospitalization for WHF by $18 \%$ compared with placebo in all SHIFT (hazard ratio $[\mathrm{HR}]=0.82 ; 95 \%$ confidence interval $[\mathrm{Cl}]=0.75,0.90 ; p<0.0001$ ), and by $15 \%$ in the $A N G$ subgroup $(H R=0.85 ; 95 \% \mathrm{Cl}=0.73,1.00 ; p=0.0553)$. Ivabradine impact on components of CV death and hospitalization for WHF were similar in all SHIFT and ANG.

Ivabradine reduced CV death or nonfatal MI by $11 \%$ versus placebo in SHIFT (HR $=0.89 ; 95 \%$ $\mathrm{Cl}=0.79,1.01 ; \mathrm{p}=0.0660)$, and by $8 \%$ in $\mathrm{ANG}(\mathrm{HR}=0.92 ; 95 \% \mathrm{Cl}=0.75,1.12 ; \mathrm{p}=0.3797)$. All SHIFT and ANG had directionally similar results for nonfatal MI, HRSHIFT $=0.93(95 \% \mathrm{Cl}=0.67$, 1.30) and $\mathrm{HRANG}=0.86(95 \% \mathrm{Cl}=0.53,1.40)$.

Conclusion: Ivabradine consistently reduced CV death or WHF hospitalization in SHIFT and in SHIFTANG; no ivabradine-related increase in nonfatal MI was apparent.

No conflict of interest 


\title{
BASIC RESEARCH, MOLECULAR BIOLOGY
}

\section{ATF3-DEPENDENT CROSS-TALK BETWEEN CARDIOMYOCYTES AND MACROPHAGES PROMOTES CARDIAC MALADAPTIVE REMODELING}

\author{
A. Aronheim, L. Koren \\ The B. Rappaport Faculty of Medicine- Technion-Israel Institute of Technology, \\ Department of Cell Biology and Cancer Science, Haifa, Israel
}

\begin{abstract}
Rationale: Pressure overload induces adaptive remodeling processes in the heart. However, when pressure overload persists, adaptive changes turn into maladaptive alterations leading to cardiac hypertrophy and heart failure. ATF3 is a stress inducible transcription factor that is transiently expressed following neuroendocrine stimulation. However, its role in chronic pressure overload dependent cardiac hypertrophy is currently unknown.
\end{abstract}

Objective: To study the role of ATF3 in chronic pressure overload dependent cardiac remodeling processes.

Methods and Results: Pressure overload was induced by phenylephrine mini-osmotic pumps in various mice models of whole body, cardiac specific, bone marrow specific and macrophage specific ATF3 ablation. We show that ATF3-KO mice exhibit a significantly reduced expression of cardiac remodeling markers following chronic pressure overload. Consistently, the lack of ATF3 specifically in either cardiomyocytes or bone marrow derived cells blunts the hypertrophic response to phenylephrine infusion. A unique cross-talk between cardiomyocytes and macrophages was identified. Transient ATF3 expression in cardiomyocytes is responsible for the induction of an inflammatory response leading to macrophage recruitment to the heart. Adoptive transfer of Wild type macrophages, but not ATF3-KO derived macrophages, into wild type mice induces maladaptive response to phenylephrie infusion.

Conclusions: Collectively, this study places ATF3 as a key regulator in promoting pressure overload induced cardiac hypertrophy through a cross-talk between cardiomyocytes and macrophages. Inhibiting this cross-talk may serve as a useful approach to blunt maladaptive remodeling processes in the heart.

No conflict of interest 


\title{
A NOVEL HYBRID MECHANICAL-TEXTURE BASED FEATURE FOR DETECTION OF HIGH- RISK ATHEROSCLEROTIC PLAQUES IN SEQUENTIAL INTRAVASCULAR ULTRASOUND FRAMES
}

\author{
A. Kermani ${ }^{1}$, A. Taki ${ }^{2}$, A. Ayatollahi ${ }^{1}$ \\ ${ }^{1}$ Iran University of science and technology IUST, electrical Engineering, Tehran, Iran \\ ${ }^{2}$ Technical University of Munich, Computer Aided Medical Procedures Group, Munich, Germany
}

Aims: The purpose of this study was to define new texture based feature to quantify one of major mechanical properties in sequential intravascular ultrasound frames (IVUS) without any extra Module. The changing of stress during the cardiac phase causes different deformation of plaque structure. As a result, a plaque appears different in two consecutive IVUS frames because of different stresses.

Method: The start and the end frames of a specific plaque are determined by an expert in an IVUS set. Then, coronary rigid motion is extracted. Next, the cardiac phase is calculated by means of spatial-intensity information during the frame interval. The elasticity of a plaque is quantified by measuring the change of normalized local intensity variation of sequential frames (Fig.1).

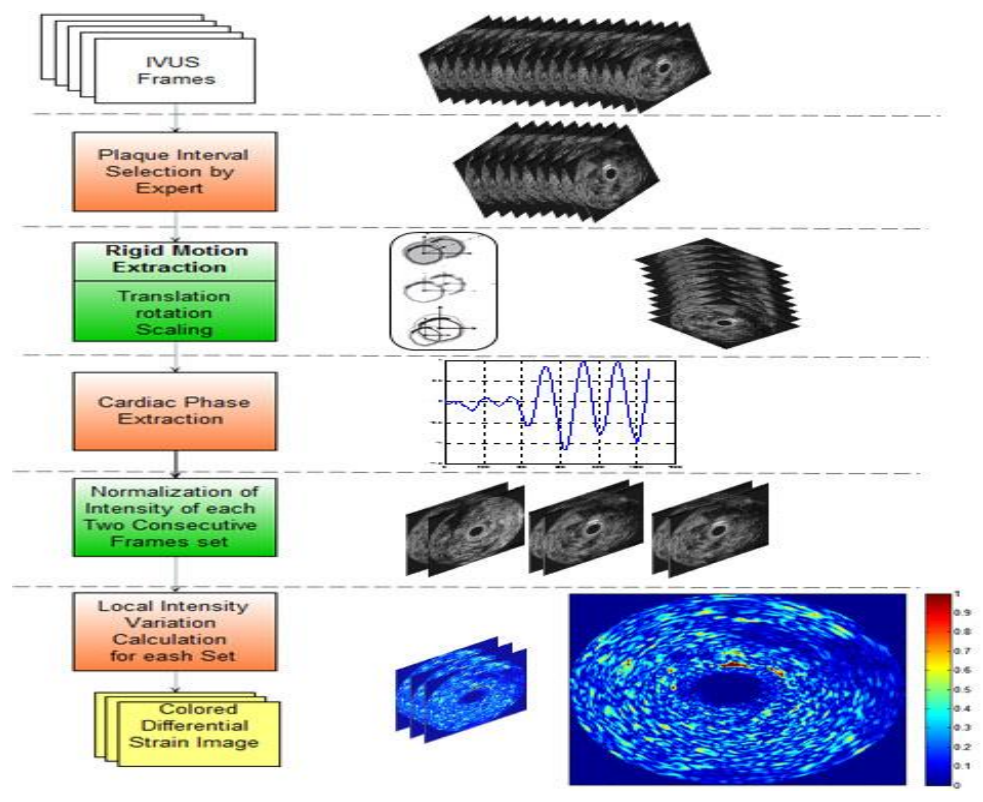

Results: The method was evaluated on an 1800 frames set of IVUS from ten patients. Three plaque classes were manually determined by an expert: calcific, fibrotic, and fibrocalcific. In all, the average of area accuracy (standard deviation) of calcific, fibrotic, and fibrocalcific Plaques compared with manual extraction were obtained $72.4 \%$ (8.1\%), $85.1 \%(4.3 \%), 78 \%(5.7 \%)$, 
respectively. the mean (standard deviation) of normalized differential strain of these three kind of plaques were associated with $.51(.13), .82(.08), .68(0.11)$, respectively.

Conclusion: Due to different elastic property of high-risk atherosclerotic plaques, they can be categorized by means of the proposed normalized differential strain feature.

No conflict of interest 


\title{
BASIC RESEARCH, MOLECULAR BIOLOGY
}

\section{INCREASE THE PREDICTIVE CAPACITY OF CORONARY RISK WITH A GENETIC SCORE}

\author{
M.I. Mendonca ${ }^{1}$, A. Pereira ${ }^{1}$, R. Rodrigues ${ }^{1}$, A.I. Freitas ${ }^{1}$, M. Neto ${ }^{1}$, A.C. Sousa ${ }^{1}$, S. Borges ${ }^{1}$, \\ S. Freitas ${ }^{1}$, R. Palma dos Reis ${ }^{2}$ \\ ${ }^{1}$ Funchal Hospital Centre, Research Unit, Funchal, Portugal \\ ${ }^{2}$ New University of Lisbon, Cardiology, Funchal, Portugal
}

Aims: Evaluate the ability of a multifactorial genetic risk score (GRS) be able to add predictive power, for the development of CAD, to the model developed only with TCRF.

Method: A case-control study was performed with 1321 coronary patients (mean age $53.4 \pm 8.1$ years, $78.8 \%$ male) and 1148 controls. Traditional risk factors (hypertension, diabetes, dyslipidemia, smoking, obesity, sedentary lifestyle, family history) were evaluated according to the International criteria. The genetic variants were analyzed with specific primers and the GRS was determined in the entire population, based on 29 genetic polymorphisms previously associated with atherosclerotic disease in general and, in particular, with CAD. A multiplicative model was then used based on risk multiplication (odds ratio - OR) of each genotype of the 29 studied genes. Subsequently, a multivariate analysis was done with the TCRF only or the TCRF with the GRS and a ROC curve was constructed for both situations. Pairwise comparison of the two ROC Curve was done by the DeLong test.

Results: After multivariate analysis, the GRS was found to be an independent predictor for CAD $(\mathrm{OR}=2.1 ; \mathrm{Cl}: 1.7-2.5 ; \mathrm{p}<0.0001)$. The AUC increased from 0.71 to 0.74 after the inclusion of $\mathrm{GRS}$ to the TCRF in the multivariate analysis (Figure).

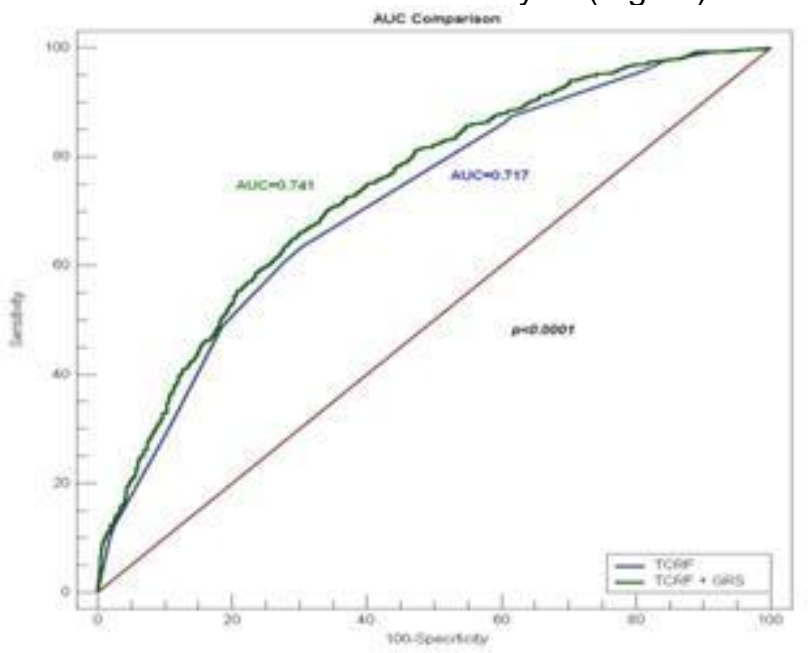

Conclusion: In our population, the multiplicative GRS was an independent predictor for CAD. When analyzed together with traditional risk factors, it adds little predictive value. Its usefulness, in clinical practice, may be directed to the intermediate risk group, in which a possible risk reclassification can have different therapeutic measures. 
No conflict of interest 


\title{
BASIC RESEARCH, MOLECULAR BIOLOGY
}

\section{GENETIC ASSOCIATION OF RS11279109 POLYMORPHISM WITH INCREASED RISK OF CORONARY HEART DISEASE IN KUWAITI NATIVES}

\author{
S. Albustan ${ }^{1}$, F. Ismael' ${ }^{1}$, A. Alserri', I. Alrashdan ${ }^{3,4}$ \\ ${ }^{1}$ Kuwait University- Faculty of Science, Biological Sciences, Safat, Kuwait \\ ${ }^{2}$ Kuwait University- Faculty of Medicine, Pathology, Safat, Kuwait \\ ${ }^{3}$ Kuwait University- Faculty of Medicine, Medicine, Safat, Kuwait \\ ${ }^{4}$ Kuwait Chest Hospital, Interventional Cardiology, Shuwaikh, Kuwait
}

\begin{abstract}
Aims: Coronary Heart Disease (CHD) is among the leading cause of death in Kuwait. CHD is considered a multifactorial disease since both environmental and genetic factors have been identified in its manifestation. Several genetic studies have implicated the association of various single nucleotide polymorphisms (SNP) to increase the risk of CHD including the $A P O B$ signal peptide rs 11279109 polymorphism. The objective of this study was to investigate genetic association of this SNP with CHD among Kuwaiti nationals employing a case-control approach.
\end{abstract}

Method: Direct amplification of the target sequence spanning the signal peptide variant was carried out to genotype a total of 735 Kuwaiti samples with documented phenotypic data. Chisquare test, univariate and logistic regression analysis were used to assess the genetic association with CHD.

Results: Genotype and allele frequency distribution revealed a significant positive association $(P<0.001)$ between rs11279109 $D$ allele and CHD in which patients showed a higher frequency $(0.32, n=372)$ than controls $(0.23, n=363)$. Moreover, logistic regression analysis revealed at least a two-fold increase for Kuwaiti carriers of the DD genotype.

Conclusion: The results suggest APOB signal peptide polymorphism may be used as an indicator for the increased risk to CHD in Kuwaiti Arabs. It may also explain molecular mechanisms that underline increased LDL and/or cholesterol levels, known strong predictors of arthrosclerosis. The data obtained emphasizes the need to understand the genetic and molecular basis of CHD in order to improve prognosis and help in the prevention, management and treatment of CHD.

No conflict of interest 


\title{
BASIC RESEARCH, MOLECULAR BIOLOGY
}

\section{ASSESSING THE RELATIONSHIP OF CARDIOVASCULAR RISK FACTORS WITH SHORT- INTERVAL CORONARY ARTERY CALCIUM CHANGES}

\author{
R. Cardarelli, W. Rankin \\ University of Kentucky, Family and Community Medicine, Lexington, USA
}

Aims: The aim of our study is to assess whether demographic and traditional cardiovascular (CV) risk factors are associated with 2-year changes in coronary artery calcification (CAC) measures.

Method: 571 male and female participants, ages of 45 and above, with no prior history of coronary, hepatic, or renal disease, were recruited in an US urban setting from 2006 to 2008. Participants underwent survey, physical, blood, and multi-slice computed tomography (MSCT) assessments. Repeat measures were conducted on 342 participants 2-years later. Unadjusted and adjusted regression models were performed to assess the relationship between demographic and traditional CV risk factors and log CAC 2-year changes.

Results: Baseline personal history of dyslipidemia and diabetes mellitus, having more than one chronic disease at baseline, and changes in systolic blood pressure and fasting glucose over 2years were significantly associated with log CAC changes. Visceral adipose tissue measures were also associated with an increase in log CAC measures over 2-years.

Conclusion: The impact of emerging and traditional $\mathrm{CV}$ risk factors are reflected in changes in $\mathrm{CAC}$ scores as early as 2-year from baseline measures. Such findings contribute to the literature to better understand the utility of CAC measures, especially for CV disease surveillance purposes.

No conflict of interest 


\title{
BASIC RESEARCH, MOLECULAR BIOLOGY
}

\section{AUTOMATED CARBON DIOXIDE DIGITAL ANGIOGRAPHY FOR LOWER LIMB ARTERIAL DISEASE EVALUATION: SAFETY ASSESSMENT AND COMPARISON WITH STANDARD IODINATED CONTRAST MEDIA ANGIOGRAPHY}

\author{
F. Scalise \\ Policlinico di Monza, Interventional Cardiology, Monza, Italy
}

Introduction: Carbon dioxide (CO2) has been validated as contrast agent in a large series of studies. A particular advantage of $\mathrm{CO} 2$ over iodinated contrast medium (ICM) is an absence of both nephrotoxicity and allergic reactions. The main limitation of $\mathrm{CO} 2$ angiography was the unreliability of $\mathrm{CO} 2$ injection owing to its compressibility. The manual gas injection does not permit an optimal control of the gas output. Development of an automated CO2 injector (Angiodroid $\AA$ ) has overcome these problems.

Aim: It was to compare the feasibility, safety, and diagnostic accuracy of $\mathrm{CO} 2$ digital subtraction angiography (DSA) with ICM DSA in the evaluation of lower extremity arterial disease.

Methods: DSA with both CO2 and ICM, to directly compare the two techniques, was performed on 40 consecutive lower limb ischemic patients. Sixteen females and 24 males participated in the study (mean age 71.7 years). We assessed the diagnostic accuracy of $\mathrm{CO} 2$ in identifying arterial stenosis in the lower limb with the ICM DSA used as the gold standard.

Results: The overall diagnostic accuracy of CO2 DSA was $96.9 \%$ (sensitivity $99.0 \%$; specificity 96.1\%; PPV 91.1\%; NPV 99.6\%). Tolerable minor symptoms occurred in 3 patients. No allergic reactions were noted in patients receiving the $\mathrm{CO} 2$ injection. No significant decline in renal function was observed in patients receiving $\mathrm{CO} 2$.

Conclusions: CO2 DSA is a valuable and safe alternative to traditional ICM DSA for evaluating lower extremity arterial disease. This alternative is particularly applicable in a patient population with a renal insufficiency or a known contrast allergy.

No conflict of interest 


\title{
BASIC RESEARCH, MOLECULAR BIOLOGY \\ CORRELATION BETWEEN VISCERAL FAT AND CORONARY ARTERY CALCIUM IN PATIENTS WITH METABOLIC SYNDROME
}

\author{
S.J. Hong, J.H. Park, D.S. Lim \\ Korea University Anam Hospital, Cardiology, Seoul, Korea
}

\begin{abstract}
Aims: To assess the relationship between visceral fat (VF) and coronary artery calcium (CAC), we performed visceral fat scan and coronary CT angiography (CCTA) in metabolic syndrome (MetS) cohort.
\end{abstract}

Method: From January to September 2014, among 1,000 MetS patients who were referred from 24 public health centers in Seoul, 740 patients who performed visceral fat scan (HDS-2000, Omron) were enrolled in this analysis. Fatty liver grade were measured from 0 to 3 by ultrasound. Brachial ankle pulse wave velocity (PWV), carotid intima-media thickness (IMT), Left ventricular mass index (LVMI) and Augmentation index (Alx) were also measured. We also measured CAC using CCTA.

Results: The cut-off value of high VF area was calculated $88 \mathrm{~cm} 2$ by Youden index. Incidence of MetS patients who had high VF area $(\geq 88 \mathrm{~cm} 2)$ was $36 \%(267 / 740)$ and the prevalence of men was higher than women $(53.4 \%$ vs $18.7 \%, p<0.001)$. High VF group showed higher CAC than normal VF $(11.2 \pm 43$ vs $25.6 \pm 95, p=0.025)$. The VF showed positive correlation with $P W V$ and IMT ( $p=0.028,0.019)$. LVMI, CAC, abdominal subcutaneous fat, fatty liver grade, serum glucose, TG, HDLc, TG/HDLc ratio, systolic and diastolic blood pressure were also positively correlated with VF (all of the $p<0.01$ ).

Conclusion: High VF group showed high CAC. Moreover, VF area had shown correlation with PWV, IMT, LVMI, CAC, TG/HDLc ratio, abdominal obesity, fatty liver grade and abdominal subcutaneous fat in MetS cohort. It may be considered that the VF could predict severity of CAC, arterial stiffness, atherosclerosis, insulin resistance and fatty liver in MetS.

No conflict of interest 


\title{
BASIC RESEARCH, MOLECULAR BIOLOGY
}

\section{COMPLEXITY OF CELLULAR STRESS REACTIONS INDUCED IN ENDOTHELIUM BY OXIDIZED PHOSPHOLIPIDS}

\author{
V. Bochkov \\ University of Graz, Pharmaceutical Sciences, Graz, Austria
}

\begin{abstract}
Aims: Oxidation of free and esterified PUFAs is an important mechanism generating mediators demonstrating biological activities that were not characteristic of their unoxidized precursors. Oxidation of PUFAs esterified in phospholipids generates oxidized phospholipids (OxPLs) known to accumulate in atherosclerotic vessels. OxPLs induce multiple effects potentially relevant to atherosclerosis including proinflammatory and toxic action on endothelial cells. OxPLs have been shown to activate cellular stress pathways such as unfolded protein response and electrophilic stress response. However, OxPLs also induce several biological effects that do not fit into the stereotypical action of „bad“ lipids. For example, OxPLs have been shown in vitro and in vivo to inhibit inflammation induced by agonists of toll-like receptors 2 and 4, protect endothelial barrier in lungs and induce angiogenesis.
\end{abstract}

Method: Analysis of gene and protein expression; analysis of cell survival and apoptosis; knockout animal model; mass spectrometry of OxPLs.

Results: We made an unexpected observation that addition of OxPLs to cultured endothelial cells protected them from toxic action of low-serum medium, chemical inducers of apoptosis, lysophosphatidylcholine or hydrogen peroxide. Our data suggest that at least partially these effects were mediated by production of specific growth factors and peptides acting in a paracrine manner.

Conclusion: These beneficial effects apparently represent a compensatory reaction of endothelial cells to the toxic action of OxPLs. Further study of protective feedback induced by atherogenic OxPLs can help in identification of new mechanisms of endothelial protection and potential drug targets promoting return of stressed endothelium to healthy functional state.

No conflict of interest 


\title{
TAVI AND VALVE INTERVENTIONS
}

\section{VALVE-IN-VALVE PROCEDURE FOR PATIENTS WITH DEGENERATED BIOPROSTHETIC VALVE: INDICATIONS, TECHNIQUE, AND RESULTS}

\author{
G. Schuler \\ Herzzentrum Leipzig, Leipzig, Germany
}

Background: Transcatheter aortic valve implantation (TAVI) using the Medtronic CoreValve (MCV) system might represent an alternative to conventional redo surgery in older high-risk patients with a failing aortic valve bioprosthesis.

Methods and Results: Symptomatic patients with failing aortic valve bioprosthesis, aged $\geq 65$ years with a logistic EuroSCORE $\geq 10 \%$ were considered for treatment. Local anesthesia was used to retrogradely implant the MCV system into the failing bioprosthetic valve. Clinical events were recorded and a transthoracic echocardiography was performed to evaluate the impact of MCV on hemodynamics after transcatheter aortic valve implantation. A total of 27 patients (aged $74.8 \pm 8$ years, logistic EuroSCORE of $31 \pm 17 \%$ ) were treated. In those with AS and AS and AR $(\mathrm{n}=25)$, the mean gradient declined from $42 \pm 16 \mathrm{~mm} \mathrm{Hg}$ before to $18 \pm 8 \mathrm{~mm} \mathrm{Hg}$ after MCV implantation $(P$

Conclusions: These results suggest that transfemoral MCV implantation into a wide range of degenerated aortic bioprosthetic valves-irrespective of the failure mode-is feasible, safe, and improves hemodynamics in older patients with higher risk for conventional aortic valve redo surgery.

No conflict of interest 


\title{
TAVI AND VALVE INTERVENTIONS
}

\section{CURRENT LIMITATIONS AND FUTURE DIRECTIONS OF TRANSCATHETER VALVULAR INTERVENTIONS}

\author{
A. Segev \\ Chaim Sheba Medical Center, The Heart Center, Tel Hashomer, Israel
}

Transcatheter aortic valve implantation (TAVI) has emerged as an evidence-based excellent therapy for high-risk patients with severe aortic stenosis. The most current study using the selfexpanding valve (US Pivoltal Study) has shown long-term mortlaity benefit for patients undergoing TAVI as compared to surgical AVR. This was also true for lower risk patients as assessed by STS below 7\%. Current limitations of TAVI include up to $5 \%$ of moderate to severe para-valvular leak (PVL), 10-20\% risk for permanent pacing.

The newer generation transcatheter valves, sapien-2 and Evolute $R$, show excellent clinical results, reduced vascular complications (14F), and much less rates of PVL.

Off-label indications that can be successfuly treated by TAVI, with growing clinical

evidence, include: bicupid aortic valves, pure aortic regurgitation, and failed aortic and mitral bioprosthetic valves.

The mitral valve in the next great frontier in structural heart interventions. While MitralClip has been used for many years with great success (now over 25000 cases world-wide), there are many companies in different stages of development of a full transcatheter mitral valve, either in a trans-apical route or trans-septal route. Some companies have completed FIM studies. Moreover, mitral valve repair by annular rings is also available. Lastly, the tricuspid valve has also being addressed percutanously by severla companies.

Conflict of Interest Disclosure Statement:

Proctor and Consultant: Edwards, Medtronic 
45

\title{
EPIDEMIOLOGY AND PREVENTION
}

\section{ROLE OF STATINS FOR CARDIAC PROTECTION: BASIC MECHANISMS}

\author{
Q. Chen, J. Strom \\ University of Arizona, Department of Pharmacology, Tucson, USA
}

Aims: The discovery of statin drugs has dramatically reduced the incidence of atherosclerosis. Statins have been marketed as inhibitors of HMG-CoA reductase, the rate-limiting enzyme that catalyzes HMG-CoA to mevalonic acid in the biosynthesis of cholesterol, therefore reducing cholesterol and LDL levels in the blood. In recent years, statins have been found to exhibit pleiotropic effects beyond cholesterol-lowering. We investigate the cardiac protective effect of statins independent of cholesterol lowering.

Method: Literature review, review of clinical trials, and animal and cell culture studies

Results: Clinical trials have demonstrated that acute administration of statins in patients suffering a myocardial infarct results in reduced morbidity and mortality independent of cholesterollowering effects. A retrospective study of 13 clinical trials has demonstrated that administration of high doses of statins as a pretreatment to percutaneous coronary intervention (PCl) reduced periprocedural myocardial infarction and 30 days major adverse cardiac events by $46 \%$. We have found that acute statin administration reduces infarct size in a surgical model of myocardial infarction independent of dyslipidemia in rodents. We have found that statins induce cytoprotective genes in the myocardium and cells in culture.

Conclusion: The short time frame of the therapeutic benefit observed with statin administration in myocardial infarct patients is inconsistent with the time course for cholesterol lowing effect. Induction of cytoprotective genes in the experimental animals and at the cellular level is important for mediating the cardiac protective effect of statins.

No conflict of interest 
48

\title{
EPIDEMIOLOGY AND PREVENTION
}

\author{
DYSLIPEMIA IN DIABETICS TREATED WITH STATINS: RESULTS OF THE DYSIS STUDY IN \\ SPAIN \\ J. Millan Núñez-Cortés \\ Catedrático-Jefe de Servicio de Medicina Interna, \\ Jefe de Estudios. Presidente de la Comisión de Docencia,Hospital General Universitario Gregorio \\ Marañón, Facultad de Medicina de la Universidad Complutense, Madrid, Spain
}

Background and Objective: Type 2 diabetes mellitus (DM2) is characterized by carrying a high cardiovascular risk. This situation underscores the importance of intensively treating the risk factors present in diabetic patients, notably dyslipemia. The treatment with cholesterol-lowering drugs may be especially effective to reduce the cardiovascular risk in diabetic patients. Therefore, LDL-cholesterol is a priority target in the lipid management of these patients. This study analyzes the alterations in the lipid profile of diabetic patients receiving treatment with statins, which therefore may contribute to persistent cardiovascular risk in such individuals.

Patients and Methods: The DYSIS (Dyslipidemia International Study) is an international, observational trial analyzing the lipid profile of patients treated with statins and followed-up on in outpatient clinics by primary care physicians and specialists. This study is referred to the data on the diabetic patients.

Results: Of the total patients enrolled in the DYSIS, the present study included 3703 patients, $39 \%$ being diabetics. A total of $59.2 \%$ of diabetics showed LDL-C out of goal; triglyceride elevation was observed in $43.6 \%$ and $36.4 \%$ showed low HDL-C. In diabetics patients with coronary heart disease, $31 \%$ had uncontrolled levels of all three lipid parameters. The prevalence of out of goal LDL-C in diabetic patients with metabolic syndrome was close to $60 \%$; $39.8 \%$ had low levels of HDL-C and $46,6 \%$ high levels of triglycerides. In addition, $57 \%$ of diabetic patients with obesity showed LDL-C out of control, despite statins treatment.

Conclusions: Cardiovascular diseases remain the main cause of morbidity-mortality in patients with DM2. The results of the present study show that in diabetic patients the degree of control is very limited with regard to LDL-cholesterol. More than half of diabetic patients treated with statins had LDL-cholesterol out of goal. The level of dyslipidemia control was low, despite statins treatment. Therefore, the detection of atherogenic dyslipidemia may point to the advisability of using combination therapy for dyslipidemia in diabetic patients.

No conflict of interest 
49

\title{
EPIDEMIOLOGY AND PREVENTION \\ PHARMACOLOGICAL APPROACHES TO THE PREVENTION OF DIABETES-INDUCED VASCULAR DYSFUNCTION
}

\author{
O.L. Woodman \\ School of Medical Sciences, RMIT University, Bundoora, Vic, Australia
}

Aims: A major cause of morbidity in diabetes is dysfunction of the vasculature and in particular impairment of endothelial function. The aim of this study was to investigate the effects of diabetes on mediators of endothelium-dependent relaxation ie nitric oxide (NO), endothelium-dependent hyperpolarisation (EDH).

Method: We examined the contribution of $\mathrm{NO}$ and EDH to endothelium-dependent relaxation of mesenteric arteries from rats with streptozotocin-induced type 1 diabetes.

Results: Diabetes causes an increased release of superoxide associated with uncoupling of eNOS and increased expression of NADPH oxidase 2 (Nox2). The increase in oxidative stress indicates a potential therapeutic target which we have investigated using 3',4'-dihydroxyflavonol (DiOHF), an effective oxygen radical scavenger. We demonstrated that arteries isolated from rats with type 1 or type 2 diabetes show an improved level of endothelium-dependent relaxation when exposed to DiOHF in vitro. Further, DiOHF treatment of rats with type 1 diabetes also improved endothelium-dependent relaxation, accompanied by decreased vascular superoxide release, increased expression of eNOS as a dimer and reduced expression of Nox2. Interestingly linagliptin, an inhibitor of dipeptidyl dipeptidase 4, also improves endothelial function when impaired by exposure to elevated levels of glucose or in arteries from type 1 diabetic rats. Linagliptin induced improvement of endothelial function, without changing glucose levels, is accompanied by a decrease in oxidant stress and indicates an additional mechanism by which linagliptin may be of therapeutic benefit in diabetes.

Conclusion: Reducing vascular oxidant stress may be an effective means to limit diabetesinduced cardiovascular morbidity and mortality.

Key words: Diabetes, endothelium

No conflict of interest 
53

\title{
CELLS, VESICLES, MOLECULES \\ ENDOTHELIUM IN ACUTE CORONARY SYNDROME
}

\author{
A.M.C. Shpektor \\ Moscow State University of Medicine and Dentistry, Moscow, Russia \\ Endothelial function plays a pivotal role in all stages of atherosclerosis, in particular by regulating \\ inflammation, vascular tone, platelet activity, blood clotting, and the state of fibrinolysis. Moreover, \\ endothelium is involved in the synthesis of NO, a powerful vasodilator agent. Also, endothelial \\ cells activate the fibrinolytic system, releasing TPA, the main plasminogen activator. This can \\ explain the value of the flow-mediated dilation (FMD), a test for assessment of endothelium \\ function. It has been demonstrated that results of the FMD test correlate with the prognosis in \\ healthy persons and in patients with coronary artery disease. In the presentation data on \\ significance of the FMD test for STEMI patients will be discussed. Although it is important to use \\ FMD to obtain prognostic data, our means of influencing actual states of the endothelium in \\ patients with acute $\mathrm{Ml}$ to improve their prognosis remain to be limited. A large part of the \\ presentation will be devoted to the analysis of the data on the attempts to improve the \\ endothelium function and thus to improve the prognosis. These attempts include pre-treatment \\ with ACE inhibitors and statins. In emergency situation the most useful course of action seems to \\ be remote ischemic preconditioning (RIPC), cycles of brief ischemic episodes. This method \\ improves endothelial function and prognosis in STEMI patients and results in partial myocardial \\ protection. However, in spite of the 20-year history of RIPC the basic mechanisms of these \\ phenomena are poorly understood. The latest data on these mechanisms will be discussed.
}

No conflict of interest 
54

\section{CELLS, VESICLES, MOLECULES}

\section{EXTRACELLULAR VESICLES AND ATHEROSCLEROSIS}

\section{Margolis}

National Institute of Child Health and Human Development, National Institutes of Health, Bethesda, MD, USA

Cells in vivo and ex vivo release membrane vesicles. Until recently, these extracellular vesicles (EVs) were considered to be "cellular dust" and did not attract much attention. However, in recent years it has become clear that EVs constitute an important physiological system of cell-cell communication. Together with two other systems of intercellular communication, namely cell-cell contact interactions and the release of soluble molecules (cytokines), EVs coordinate normal body physiology and are altered in various pathologies. By incorporating cellular proteins into their membranes, EVs may reflect both the types of the cells from which they were released and the physiological state of these cells, including pathological changes. Therefore, EVs are now widely studied as candidates for diagnostic markers and are often referred to as "liquid biopsies". A significant body of evidence has been accumulated regarding EV changes in cardiovascular diseases, in particular in atherosclerosis. Although EVs are very diverse in size and composition and are pooled in blood, they are still analyzed predominantly in bulk. This presentation will focus on the current methods of EV analysis and on the relation of EVs of different types with different cardiovascular diseases, in particular with atherosclerosis. Future analysis of the antigenic composition of individual EVs may lead to the development of new diagnostic tools.

No conflict of interest 


\title{
55
}

CELLS, VESICLES, MOLECULES

\section{HERPESVIRUSES AND ACS}

\author{
E.V.C. Vasilieva \\ Moscow State University of Medicine and Dentistry, Moscow, Russia
}

Herpesviruses (HHVs) are among the most ancient human viruses that are now attracting attention in various field of biomedicine. These viruses are possible candidates for being important triggers of inflammatory processes that are associated with various human pathologies. Indeed, HHVs are highly prevalent in the population and do not enter the complete latency but rather are often reactivated. In particular, cytomegalovirus (CMV) is one of the candidates for triggering systemic and/or local inflammation that leads to the acute coronary syndrome (ACS). Although, in animal models it was demonstrated that herpesviruses are capable to contribute to the development of atherosclerosis-type pathologies, data on the involvement of HHVs in human ACS are far less conclusive, in part, because the published works focused predominantly on HHV serology (indicating merely an earlier infection), rather than on virus reactivation at the time of the acute coronary episode. In the presentation, the relation between the presences of HHVs in serum, that evidences of HHV actual reactivation, and the clinical status of patients with atherosclerosis will be discussed. In particular, evidences of strong correlations between local immunoactivation of $\mathrm{T}$ lymphocytes in atherosclerotic plaques, increased levels of C-reactive protein and the acute coronary syndrome will be presented, and the causative relationship between the HHV infection and ACS will be discussed.

No conflict of interest 


\section{CELLS, VESICLES, MOLECULES}

\section{MOLECULAR MECHANISMS OF MYOCARDIAL PROTECTION: ROLE OF NRF2 GENE}

\section{Q. Chen}

University of Arizona, Department of Pharmacology, Tucson, USA

Aims: Oxidative stress plays an important role in mitochondrial decay and cardiac injury. The paradox is that low to mild doses of oxidative stress activate endogenous defense mechanisms, including Nrf2, a transcription factor regulating the expression of a number of antioxidant and detoxification genes. In experimental animals, we found that knocking out Nrf2 gene results in an increase in cardiac injury due to myocardial ischemia, and loss of preconditioning induced cardiac protection. We address whether Nrf2 plays a role in protecting mitochondria and whether there is a role of extracellular vesicles in cardiac injury or protection.

Method: A combination of cellular and molecular biology techniques were used to determine mitochondrial morphology, mitochondrial function, mitochondrial protein expression, localization of Nrf2 protein and extracellular vesicle secretion.

Results: Normally, mitochondria in cardiomyocytes exist in elaborated networks. Following oxidative stress, mitochondria show individual punctuate morphology with disorganized cristae and area of condensation in surviving cardiomyocytes. Mitochondrial protein expression decreases in company with decreased functionality. Nrf2 overexpression prevents oxdiants from inducing mitochondria morphological changes or decline in mitochondrial function. Interestingly, Nrf2 protein was found to associate with the outer mitochondrial membrane. Mitochondria prepared from the myocardium of Nrf2 knockout mice are more sensitive to permeability transition.

Conclusion: Our finding of Nrf2 association with mitochondria provides an additional mechanism for the cardiac protective funciton of Nrf2 gene.

No conflict of interest 


\title{
CELLS, VESICLES, MOLECULES
}

\section{FUNCTION OF ABCA1 AND ABCG1 IN EXTRACELLULAR CHOLESTEROL DEPOSITION BY MACROPHAGES}

\author{
X. Jin, $\underline{\text { H. Kruth }}$ \\ NHLBI- National Institutes of Health, Experimental Atherosclerosis Section, Bethesda, USA
}

\begin{abstract}
Aims: Atherosclerotic plaques contain cholesterol in both intracellular and extracellular forms. However, the origin and fate of extracellular cholesterol in plaques is far less understood compared with intracellular cholesterol. ABCA1 and ABCG1 are two proteins that mediate cholesterol efflux from macrophages. The aim of our study was to determine whether these proteins also function in macrophage deposition of cholesterol into the extracellular matrix.
\end{abstract}

Method: Deposition of cholesterol into the extracellular matrix by cholesterol-loaded macrophages was detected using a unique monoclonal antibody, which labels lipid particles enriched with unesterified cholesterol.

Results: $L X R$ is a transcription factor that upregulates gene expression of $A B C A 1$ and ABCG1, and probucol is an inhibitor of ABCA1. The LXR agonist, TO901317 increased and probucol inhibited macrophage deposition of cholesterol into the extracellular matrix, consistent with ABCA1 functioning in extracellular cholesterol deposition. Studies of macrophage extracellular cholesterol deposition by ABCG1 and ABCA1-deficient mouse macrophages showed that both $A B C A 1$ and $A B C G 1$ mediate macrophage deposition of cholesterol into the extracellular matrix. The extracellular cholesterol deposits can be mobilized by cholesterol acceptors such as HDL, thus linking these extracellular cholesterol deposits with the reverse cholesterol transport pathway.

Conclusion: Macrophages not only clear their excess unesterified cholesterol by cholesterol esterification and storage within lipid droplets, but also by depositing this excess cholesterol within the extracellular matrix, a process that depends on ABCA1 and ABCG1. Because buildup of excess unesterified cholesterol within cells can be cytotoxic, extracellular deposition of cholesterol represents another means by which macrophages maintain cholesterol homeostasis.

No conflict of interest 


\title{
CELLS, VESICLES, MOLECULES
}

\section{INFLAMMATION AS A THERAPEUTIC TARGET IN CARDIOVASCULAR DISEASE}

\author{
R. Ritchie \\ Baker IDI Heart and Diabetes Institute, Melbourne, Australia
}

\begin{abstract}
Aims: Development of new therapeutic strategies for treating myocardial infarction (MI) after the event (while the injury is still evolving), alone or concurrent with standard care, is urgently warranted. Thrombolysis and percutaneous revascularization interventions restore blood flow to ischaemic tissue to prevent tissue necrosis and preserve organ function in the very short-term (hours). Myocardial injury however continues to evolve over days-weeks post MI, with adverse cardiac remodelling and cardiac contractile dysfunction ultimately progressing to heart failure (HF) and death. Targeting annexin-A1, an endogenous glucocorticoid-regulated anti-inflammatory mediator, may represent one such potential therapeutic approach.
\end{abstract}

Methods: These studies used a pre-clinical mouse model of MI, occlusion of the left anterior descending coronary artery, in both wild-type and annexin-A1-deficient mice, \pm treatment with the annexin-A1 peptide mimetic Ac2-26 (1mg/kg/day), for up to 4 weeks.

Results: Our laboratory provided the first evidence of the direct protective annexin-A1 actions on myocardium, independent of inflammatory cells in cardiomyocytes and isolated hearts in vitro. More excitingly, our laboratory is also the first to now demonstrate reductions in each of cardiac necrosis at $24 \mathrm{~h}$, cardiac inflammation at $48 \mathrm{~h}$, and cardiac fibrosis at 7 days after myocardial ischaemic insult, in mice in vivo. Preservation of cardiac contractile function is also observed up to 4 weeks after MI. In contrast, annexin-A1 deficient mice exhibit an exaggerated response to each of these endpoints in vivo.

Conclusion: Annexin-A1 thus represents a novel modulator of myocardial viability and contractile function for rescuing the chronically ischaemic heart in vivo, and represents a valuable and novel target for improving outcome after MI.

No conflict of interest 


\title{
CELLS, VESICLES, MOLECULES
}

\section{INTRAVENOUS VEPOLOXAMER (PURIFIED POLOXAMER-188) FOR THE TREATMENT OF HEART FAILURE}

\author{
H.N. Sabbah \\ Division of Cardiovascular Medicine, Department of Medicine, Henry Ford Hospital, Detroit, MI, \\ USA
}

\begin{abstract}
Aims: Calcium overload occurs in heart failure (HF) leading to cardiomyocyte dysfunction and loss. Vepoloxamer (VEPO), purified poloxamer-188, is a rheologic agent that can improve microvascular blood flow and/or repair damaged cell membranes, possibly inhibiting unregulated calcium entry into cardiomyocytes. The objective of our studies was determine the effects of VEPO on LV function in HF dogs.
\end{abstract}

Method: Studies were performed in dogs with HF produced by coronary microembolizations and in isolated cardiomyocytes from HF dogs.

Results: In a proof of concept study in HF dogs, a 2 hour intavenous infusion of VEPO, reduced LV end-systolic volume (ESV) and increased LV ejection fraction (EF) without affecting heart rate or mean aortic pressure. VEPO-treated dogs showed reduced plasma levels of troponin-I, nt-pro brain natriuretic peptide, tumor necrosis factor-alpha, interlukin- 6 and c-reactive protein compared to controls. These benefits tended to persist for at least one week after the end of infusion. Similar benefits were seen in a second study also in HF dogs in which multiple infusions of VEPO were administered 3 weeks apart. The benefits were sustained up to at least 2 weeks after the 1 st VEPO infusion and up to 3 weeks after the 2nd VEPO infusion. Studies in isolated failed cardiomyocytes treated with VEPO showed a dose-dependent reduction in intracellular calcium concentration.

Conclusion: The results studies indicate that intravenous infusions of VEPO elicit progressive improvement in LV function. The findings support development of VEPO for the treatment of acute HF and potentially for continued therapy in patients with HF post-hospital discharge.

Conflict of Interest Disclosure Statement:

Research Grants from Mast Therapeutics, Inc., Consultant to Mast Therapeutics, Inc. 
64

SURGICAL ASPECTS: NEW CHALLENGES IN CARDIAC SURGERY

ROBOTIC EXPERIENCE WITH MITRAL VALVE REPAIR

A. Trento $^{1}$, D. Ramzy' ${ }^{2}$, M. De Robertis ${ }^{2}$, J. Mirocha ${ }^{3}$, W. Cheng 4 , L. Czer ${ }^{5}$

${ }^{1}$ Cedars-Sinai Medical Center, Division of Cardiothoracic Surgery, Los Angeles, USA

${ }^{2}$ Cedars-Sinai Medical Center- Heart Institute, Division of Cardiothoracic Surgery, Los Angeles, USA

${ }^{3}$ Cedars-Sinai Medical Center- Heart Institute, Biostatistics, Los Angeles, USA

${ }^{4}$ Cedars-Sinai Medical Center- Heart Institute, Division of Cardiothoracic, Los Angeles, USA

${ }^{5}$ Cedars-Sinai Medical Center- Heart Institute, Cardiology, Los Angeles, USA

Aims: To review our 10 year robotic assisted mitral valve repair (R-MVr) procedure outcomes comparing our previously reported cases with our most recent.

Method: From June 2005 to August 2015, we performed 534 R-MVr. Cases were divided into 2 Eras, Era-1 our first 300 cases, Era-2 our latest 234. All consecutive patients needing isolated MVr underwent R-MVr.

Results: All 534 patients had preoperative severe mitral regurgitation (MR). Patients were older in Era-2 ( $59.3 \pm 11.0$ vs. $62.9 \pm 12.2$ years, $p=0.0003)$. No other differences were found in preoperative characteristics. There was more anterior leaflet prolapse in Era-1 vs Era-2 (28\% vs $19.7 \%, p=0.007$ ) and no difference in posterior prolapse, Barlow syndrome, or ruptured chordae. Pump-time minutes decreased from Era-1, 144.6 \pm 37.2 to $126.1 \pm 32.4$ in Era-2 $(p=0.0001)$. Cross-clamp time decreased from Era-1, 101.6 \pm 28.5 minutes to $79.2 \pm 22.7$ in Era-2 $(p=0.0001)$. There were no differences in postoperative bleeding, infection, thoracentesis, atrial fibrillation, dialysis or stroke. One hospital death $(0.33 \%)$ occurred in Era-1 and none in Era-2. There were $14 \mathrm{MV}$ reoperations, $13(4.3 \%)$ vs $1(0.4 \%)(\mathrm{p}=0.005)$, Era-1 vs Era-2 respectively. Ten $(1.9 \%)$ required subsequent MV replacement, Era-1, 9 (3.0\%), 1 (0.4\%) in Era-2. Four (1.3\%) re-repairs were performed in Era-1, and none in Era-2. None to mild post-pump MR was $80.0 \%$ vs $100 \%$ $(\mathrm{p}=0.0001)$, Era-1 vs Era-2 respectively.

Conclusion: Complications requiring reoperations occurred early in our experience. A newer improved robotic system was introduced late in Era-1 and together with increased experience made $\mathrm{R}-\mathrm{MVr}$ for all types of degenerative mitral valve pathology reproducibly successful through time.

No conflict of interest 
65

SURGICAL ASPECTS: NEW CHALLENGES IN CARDIAC SURGERY

\title{
DOES MITRAL VALVE ANNULOPLASTY IMPROVE LONG-TERM SURVIVAL IN PATIENTS HAVING MODERATE ISCHEMIC MR UNDERGOING CABG?
}

\author{
S. Silberman, O. Merin, A. Alshosha, A. Butnaru, S. Shahar, R. Tauber, D. Bitran, D. Fink \\ Department of Cardiothoracic Surgery, Shaare Zedek Medical Center, Jerusalem, Israel
}

Introduction: The surgical approach to patients with moderate ischemic MR undergoing CABG is as yet undetermined. We examined long term outcomes in patients with moderate IMR undergoing $\mathrm{CABG}$ and compared outcomes between those undergoing a restrictive annuloplasty to those having no intervention on the mitral valve.

Methods: A retrospective analysis of our database revealed 231 patients operated between 1993-2011 with moderate IMR: group $1(n=186)$ underwent isolated CABG, group $2(n=45)$ underwent $\mathrm{CABG}$ with concomitant mitral valve annuloplasty. Univariate analysis was used to compare baseline parameters. Kaplan-Meier estimates were used to compare survival. Cox multivariate regression was used to determine predictors of late survival. Survival data up to 20 years is $97 \%$ complete.

Results: The groups were similar with respect to preoperative baseline parameters including age, prior MI, LV function, and incidence of atrial fibrillation. Patients undergoing mitral repair had a higher incidence of CHF ( $<<0.0001)$. Overall operative mortality was $7 \%$ and similar between groups. Ten year survival was $53 \%$ and $47 \%$ for groups 1 and 2 respectively ( $p=0.2)$. Residual MR was less $(p<0.0001)$ and functional improvement was better in patients undergoing mitral annuloplasty $(p=0.02)$. Predictors of late mortality included age, symptoms of CHF, LV dimensions and preoperative LV dysfunction. Neither the addition of a mitral procedure, type of ring implanted nor residual MR after surgery emerged as predictors for survival. The incidence of CHF at follow-up was higher in the non-repair group $(p=0.02)$.

Conclusions: In patients with moderate IMR, neither operative mortality nor long term survival are affected by the performance of a restrictive annuloplasty, however functional class was better in patients undergoing mitral repair. The decision to repair the valve should be taken on an individual basis, taking into consideration individual patient factors. 
Figure: Long-term survival

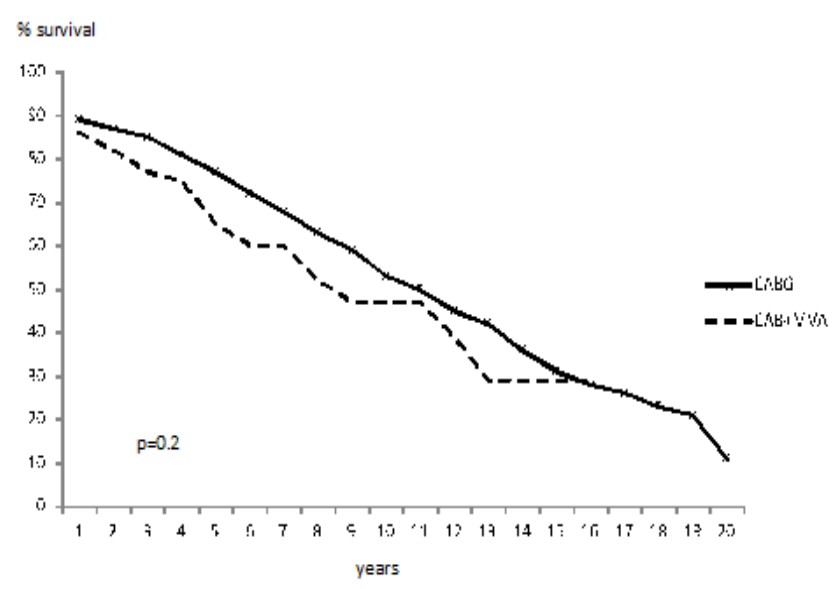

Patients at risk:

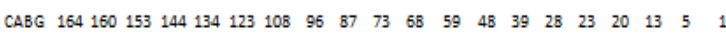

+MVA $\begin{array}{llllllllllllllll}39 & 37 & 34 & 33 & 25 & 22 & 19 & 14 & 11 & 9 & 7 & 5 & 3 & 2 & 2 & 1\end{array}$

No conflict of interest 
69

\title{
DIAGNOSTIC METHODS
}

\section{TOWARDS CORRECT IDENTIFICATION AND CLASSIFICATION OF PATIENTS WITH SUSPECTED MYOCARDIAL INFARCTION BUT WITH UNDETECTABLE HIGH-SENSITIVE CARDIAC TROPONIN}

\author{
L. Bongartz ${ }^{1}$, H. Kirkels ${ }^{1}$, J. Deckers ${ }^{2}$ \\ ${ }^{1}$ University Medical Center Utrecht, Cardiology, Utrecht, Netherlands \\ ${ }^{2}$ Erasmus Medical Center, Cardiology, Rotterdam, Netherlands
}

\begin{abstract}
Aims: The application of high-sensitive cardiac troponin (hs-Tn) will significantly impact on the correct early diagnostic and prognostic stratification of patients presenting with chest pain. We explored the value of undetectable hs-Tn at presentation, as opposed to non-elevated values which are often indicated as "negative".
\end{abstract}

Method: A Medline search was performed for studies that investigated the negative predictive value (NPV) of undetectable hs-cTn (an initial hs-Tn below the limit-of-detection [LoD]) for major adverse cardiac events (MACE) at short-term \& long-term follow up.

\begin{tabular}{|l||l||l|l|l|l||}
\hline Study, year & N & $\begin{array}{l}\text { Initial MACE } \\
\text { (all pts) }\end{array}$ & $\begin{array}{l}\% \text { of pts w/ hs-cTn } \\
\text { below LoD }\end{array}$ & $\begin{array}{l}\text { NPV for } \\
\text { initial MACE }\end{array}$ & $\begin{array}{l}\text { Late MACE (pts w/ } \\
\text { negative hs-Tn) }\end{array}$ \\
\hline \hline Body, 2015 & 463 & $17 \%$ & $17 \%$ & $100 \%$ & $0 \%$ \\
\hline Meune, 2013 & 1181 & $30 \%$ & $17 \%$ & $100 \%$ & $0 \%$ \\
\hline Thelin, 2014 & 478 & $22 \%$ & $33 \%$ & $97 \%$ & $0.6 \%$ \\
\hline $\begin{array}{l}\text { Rubini } \\
\text { Gimenez, } 2013\end{array}$ & 2072 & $23-29 \%$ & $11-27 \%$ & $98.6-100 \%$ & $0-0.6 \%$ \\
\hline $\begin{array}{l}\text { Bandstein, } \\
\text { 2014 }\end{array}$ & 14636 & $14 \%$ & $61 \%$ & $99,8 \%$ & $0.2 \%$ \\
\hline Sanchis, 2012 & 446 & $19 \%$ & $35 \%$ & $97 \%$ & Na \\
\hline Body, 2011 & 703 & $19 \%$ & $28 \%$ & $100 \%$ & $0 \%$ \\
\hline Reichlin, 2009 & 718 & $17 \%$ & unknown & $98-100 \%$ & unknown \\
\hline
\end{tabular}

Results: The presence of undetectable hs-cTn values in patients with chest pain identified a considerable proportion - about $25 \%$ - in whom MACE could be ruled out with almost $100 \%$ accuracy with excellent long term outcome.

Conclusion: In patients with chest pain at rest and non-diagnostic ECG, whether or not suspected for acute coronary syndrome, an undetectable hs-Tn virtually negates an acute myocardial infarction and MACE. Since prognosis during longer follow-up is excellent, a diagnosis of unstable angina can also be refuted in these patients.

No conflict of interest 
70

\title{
DIAGNOSTIC METHODS
}

\section{PREDICTIVE VALUE OF CFR AT STRESS-ECHO ABOUT THE PRESENCE OF CAD IN PATIENTS WITHOUT WALL MOTION ABNORMALITIES}

\author{
A. Auriti ${ }^{1}$, V. Loiaconi ${ }^{2}$, G. Speciale ${ }^{3}$, C.S. Barillà ${ }^{4}$, L. Monaco ${ }^{5}$, M. Rizzo ${ }^{5}$, F. Colivicchi ${ }^{5}$ \\ ${ }^{1}$ San Filippo Neri Hospital, Cardiovascular Disease, Rome, Italy \\ ${ }^{2}$ San Filippo Neri Hospital Rome, Cardiosurgery, Rome, Italy \\ ${ }^{3}$ San Filippo Neri Hospital Rome, Coronary Intervention Unit, Rome, Italy \\ ${ }^{4}$ San Filippo Neri Hospital Rome, Cardiology, Rome, Italy \\ ${ }^{5}$ San Filippo Neri Hospital Rome, Cardiovascular Disease, Rome, Italy
}

Aims: Recording Coronary Flow Reserve (CFR) in patients with suspected coronary artery disease (CAD) has become a routine in some echo-labs. However, the significance of a reduced CFR $(<2)$ in patients without wall motion abnormalities (WMA) at stress-echocardiography (SE) is a bit unclear. To assess the weight of CFR in patients without WMA about the presence of CAD we studied 37 patients with known or suspected CAD scheduled for coronary angiography (CA) for chest discomfort.

Method: CFR was recorded in 37 patients during SE. After dipyridamole injection $(0,84 \mathrm{mg} / \mathrm{Kg}$ in $6 \mathrm{~min}$ ), CFR was recorded mostly, not only, in the left anterior descending artery. 28 patients with reduced CFR without WMA were included. Subsequently, all patients underwent CA in order to assess the coronary anatomy.

\section{Results:}

1) $89 \%$ (25/28) of pts with reduced CFR had CAD (stenosis $\geq 20 \%)$. In this group, $60 \%(15 / 25)$ of pts had severe CAD (stenosis $>70 \%$ in 1 to 3 coronary arteries) and needed PCl or CABG.

2) Only $11 \%(3 / 28)$ of pts with reduced CFR had normal coronary arteries. PPV of CFR for CAD in patients without WMA at SE was: $89 \%(p<0.0001)$.

Conclusion: Our findings indicate a close relationship between reduced CFR and CAD, even in patients without WMA. SE with CFR recording (Dual-Test) is a good tool to select patients suitable for CA. Therefore, we suggest performing CA in patients with reduced CFR at SE.

No conflict of interest 
71

\title{
DIAGNOSTIC METHODS
}

\section{IS THERE A ROLE FOR AN IN-PATIENT CTCA SERVICE IN SELECTED PATIENTS WITH ACUTE CORONARY SYNDROME? A COHORT ANALYSIS OF IN-PATIENT TERTIARY CENTRE REFERRALS}

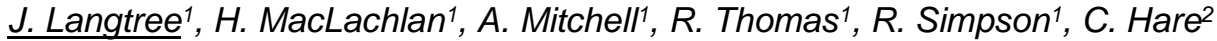 \\ ${ }^{1}$ Jersey General Hospital, Department of Cardiology, St Helier, Jersey \\ 2Jersey General Hospital, Department of Radiology, St Helier, Jersey
}

\begin{abstract}
Aims: Modern computerised tomography coronary angiography (CTCA) provides a reliable noninvasive alternative to invasive coronary angiography (ICA) to exclude coronary heart disease (CHD). The objective of this study was to conduct a retrospective analysis of in-patients referred for ICA at a tertiary centre with suspected or confirmed ACS. Primary outcome measure was evidence of $\mathrm{CHD}$ at ICA requiring intervention. The secondary aims were to calculate the total cost of in-patient referral for this sub-group and evaluate the role of local in-patient CTCA as a cost-effective alternative investigation to tertiary ICA in the context of suspected or confirmed ACS.
\end{abstract}

Method: Using GRACE scoring we identified the number of patients who did not require intervention following ICA but who also met the ESC criteria for consideration of CTCA as a primary investigation for suspected ACS.

Results: ICA demonstrated evidence of CHD requiring coronary intervention in $119(60 \%)$ of the patients referred over the three years. 79 patients $(40 \%)$ were managed medically. Of these, 64 $(81 \%)$ required no further investigation following ICA and were discharged with local follow up. The mean GRACE scores for the non-intervention and intervention group were 97 and 119 respectively $(p<0.01)$. The mean calculated in-hospital mortality risks of the non-intervention and intervention groups were $0.9 \%$ and $4.4 \%$. The mean calculated six-month mortality risks of the non-intervention and intervention groups were $2.1 \%$ and $8.1 \%$ respectively.

Conclusion: Patients presenting to hospitals without on-site ICA with suspected or confirmed ACS and low to intermediate risk GRACE scores should be considered for in-hospital CTCA.

No conflict of interest 
72

\title{
DIAGNOSTIC METHODS
}

\section{RISK-EFFECT INTEGRATION: A DIAGNOSTIC TOOL TO BETTER DETECT CAD}

\author{
L. Sgorbini, G. Adone, M. Delfini, G. Limongelli, A. Maddaluna, A. Rossetti, A. Picelli, A. Galati \\ H. S. Spirito Villa Betania, Cardiac Rehabilitation, Roma, Italy
}

Aims: Cardiovascular disease is a major health problem in Western Countries and risk calculators try to predict the gross individual probability of developing coronary artery pathologies in the future. However, there is a great difficulty to identify CAD patients due to many confounding and unrecognized risk factors. Our aim is to assess CAD by the integration of the current ESC guidelines risk score (risk) and peripheral vascular artery ultrasound examination (effect): riskeffect integration (REI).

Method: 466 patients who underwent CABG and/or valvular replacement, were consecutively admitted to our department for cardiac rehabilitation; all patients performed coronary angiography prior surgery. After, all patients performed a ultrasonographic detection of subclinical atherosclerosis of carotid, anonymous and subclavian arteries, heart, ascending, arch and abdominal aorta, common, superficial femoral arteries and bifurcations. IMT and plaque were detected (effect). CV risk SCORE was computed for each patient.

Results: $98 \%$ of patients with presence of peripheral plaques showed CAD; of these, $78 \%$ were significant. CAD patients showed risk as follows: $24 \%$ very high (10-19), $33 \%$ high $(5-7), 30 \%$ medium (2-4) and $13 \%$ low (0-1). Of $78 \%$ with significant CAD, $23 \%$ were very high risk, $35 \%$ high, $30 \%$ medium and $12 \%$ low. $96 \%$ of patients without effect showed absence of CAD while their risk was high (13\%), medium (41\%) and low (46\%).

Conclusion: These preliminary data show that risk score cannot correctly detect CAD/no CAD patients. REl could represent a new tool to improve the identification and graduation of CAD patients.

No conflict of interest 
73

\title{
DIAGNOSTIC METHODS
}

\section{ASSOCIATION OF UPRIGHT T-WAVE IN V1 LEAD AND THE PRESENCE OF CORONARY ARTERY DISEASE}

\author{
A. Shafiee ${ }^{1}$, A. Amirzadegan², E. Sekhavati ${ }^{3}$, M. Salarifar ${ }^{1}$ \\ ${ }^{1}$ Tehran Heart Center - Tehran University of Medical Sciences, Cardiovascular Research, \\ Tehran, Iran \\ ${ }^{2}$ Tehran Heart Center - Tehran University of Medical Sciences, Interventional Cardiology, Tehran, \\ Iran \\ ${ }^{3}$ Tehran Heart Center - Tehran University of Medical Sciences, Cardiology, Tehran, Iran
}

Aims: We aimed to determine the association of upright $\mathrm{T}$ wave in lead $\mathrm{V} 1$ and stenosis of coronary artery branches in patients who underwent coronary angiography at our center and had normal electrocardiogram.

Method: We retrospectively reviewed the clinical and coronary angioraphy data of patients who had normal electrocardiogram. Presence of coronary artery disease was compared between patients with and without upright $\mathrm{T}$ wave in lead V1 as well as other clinical and angiographic parameters.

Results: Data of a total of 100 patients with upright T wave in lead V1 was compared with 100 patients with completely normal electrocardiograms. Frequency of males and CAD patients were higher in the upright T wave group. However, the groups did not differ statistically as regards the cardiovascular risk factors. Based on the multivariable regression model and after adjustment for confounding factors (i.e. gender, dyslipidemia and smoking), presence of upright $T$ wave in the $\mathrm{V} 1$ lead could predict the presence of coronary artery disease (Odds ratio $=3.30,95 \% \mathrm{Cl}: 1.73$ $6.29 ; \mathrm{P}<0.001)$.

Conclusion: We suggest that the use of electrocardiogram, particularly the presence of upright $T$ wave in lead V1 could help to select the candidates for coronary angiography.

No conflict of interest 
74

\section{DIAGNOSTIC METHODS}

\section{RELATIONSHIP BETWEEN VASCULAR REMODELING AND PLAQUE COMPOSITION, EVALUATED BY INTRAVASCULAR ULTRASOUND-VIRTUAL HISTOLOGY STUDY}

Q. Tan

Qinhuangdao First Hospital, Cardiology, Qinhuangdao, China

Aims: The aim of this study was to evaluate relationship between positive vascular remodeling and plague composition in culprit coronary artery

Method: The study analyzed 41 patients with coronary artery disease that underwent intravascular ultrasound-virtual histology examination in a culprit lesion. Patients were divided into positive remodeling (PR) group or negative remodeling (NR) group according to remodeling index (RI)

Results: Patients with positive remodeling had bigger EEM area (17.95 \pm 4.25 vs $11.44 \pm 2.13$ $\mathrm{mm} 2)$, luman area $(6.02 \pm 1.85$ vs $4.98 \pm 1.06 \mathrm{~mm} 2)$ and plague burden $(63.83 \pm 7.89 \%$ vs $63.83 \pm 7.89 \%)$ compared with negative remodeling patients $(p<0.05)$; virtual histology analysis showed PR group had higher necrotic core $(30.38 \pm 9.33 \%$ vs $17.19 \pm 11.31 \%, p=0.001)$ and lower fibrous ratio $(44.61 \pm 14.93 \%$ vs $61.08 \pm 14.81 \%, p=0.002$ )than NR group. There were no significant difference in calclium area and fibrous-fatty area between the two groups. RI showed significant positive correlation with necrotic core $(r=0.373, p=0.023)$.

Conclusion: Positive remodeling in a culprit lesion is associated with the increased ratio of necrotic core. Positive remodeling is one of characteristic of plaque vulnerability.

No conflict of interest 
75

DIAGNOSTIC METHODS

EXPERIMENTAL STUDY OF EVALUATING LEFT VENTRICULAR REGIONAL SYSTOLIC FUNCTION USING SPECKLE TRACKING ECHOCARDIOGRAPHY IN ACUTE MYOCARDIAL INFARCTION MODEL OF DOGS

G. Song, R. Zhang

The First Affiliated Hospital of Zhengzhou University, Ultrasound, Zhengzhou, China

Aims: To investigate the value of three-dimensional speckle tracking imaging (3D-STI) in assessment of left ventricular regional systolic function in acute myocardial infarction model of dogs.

Method: Left anterior descending coronary artery was ligated in 24 adult beagle dogs. Longitudinal strain (LS) , circumferential strain(CS), radial strain(RS) of every left ventricular segment before ligation and $180 \mathrm{~min}$ after ligation were obtained using two-dimensional speckle tracking imaging (2D-STI) and 3D-STI. After experiment, left ventricular myocardium were determined by TTC staining and divided into two groups: infarcted myocardial segments and noninfarcted myocardial segments.

Results: Experimental model of acute myocardial infarction was established successfully in 20 dogs. After ligation, both LS, CS, RS of 2D-STI and 3D-STI in infarcted myocardial segments were significantly lower $(P<0.01)$. LS of 3D-STI absolute value was lower than 2D-STI $(\mathrm{P}<0.01)$, CS of 3D-STI absolute value was larger than 2D-STI $(P<0.01)$. After ligation, the RS of 3D-STI was lower than that of 2D-STI in infarcted myocardial segments $(P<0.05)$. The area under the ROC curve of 3D-STI were larger than that of 2D-STI. A cut-off value of $-12.56 \%$ for LS of the 3DSTI had $77.9 \%$ sensitivity and $88.2 \%$ specificity in predicting acute myocardial infarction.

Conclusion: 3D-STI is a convenient, accurate method for evaluating left ventricular regional systolic function.

No conflict of interest 
78

\title{
CELL THERAPY IN MYOCARDIAL PROTECTION \\ IMPROVING THERAPEUTIC EFFICACY OF ADIPOSE-DERIVED STEM CELLS WITH PDE5 INHIBITION
}

\author{
R. Kukreja \\ Pauley Heart Center, Virginia Commonwealth University, Richmond, VA, USA \\ We tested the hypothesis whether inhibition of c-GMP hydrolyzing enzyme, phosphodiesterase- 5 \\ (PDE5) in adipose-derived stem cells (ASCs) improves their survival and enhances cardiac \\ function following myocardial implantation in vivo. ASCs were treated with sildenafil or PDE5 \\ silencing short hairpin RNA (shRNA-PDE-5)) and subjected to simulated ischemia/reoxygenation \\ in vitro. Both sildenafil and shRNA-PDE-5 significantly improved viability, decreased necrosis, \\ apoptosis, and enhanced the release of growth factors, vascular endothelial growth factor \\ (VEGF), basic fibroblast growth factor (b-FGF), and insulin-like growth factor. Inhibition of protein \\ kinase $G$ reversed these effects. To show the beneficial effect of preconditioned ASCs in vivo, \\ adult male CD-1 mice were subjected to myocardial infarction and ASCs $(4 \times 105)$ were directly \\ injected intramyocardially. The results show that preconditioned ASCs-treated hearts had \\ consistently superior cardiac function when compared with the non-preconditioned ASCs after 4 \\ weeks of treatment. This was associated with significantly reduced fibrosis, increased vascular \\ density, and decreased resident myocyte apoptosis when compared with mice receiving non- \\ preconditioned ASCs. VEGF, b-FGF, and Angiopoietin-1 were also significantly elevated 4 weeks \\ after cell therapy with preconditioned ASCs. We conclude that preconditioning by inhibition of \\ PDE5 can be a powerful approach to improve stem cell therapy following myocardial infarction.
}

No conflict of interest 


\title{
CELL THERAPY IN MYOCARDIAL PROTECTION \\ PROGENITOR CELLS FROM MASSETER MUSCLE CONTAINS CELLS THAT CAN DIFFERENTIATE INTO BEATING CARDIOMYOCYTES
}

\author{
Y.-G. Wang \\ Department of Pathology and Laboratory Medicine, University of Cincinnati, OH, USA
}

Background: Induced pluripotent stem cell (iPSC) can efficiently differentiate into functional cardiomyocytes through embryoid bodies (EBs) in the presence of specific signals. Recently, it has been suggested that electrical stimulation (EleS) can enhance potency of proliferation and differentiation in various stem cells. This study investigated the effects of EleS on human iPSC differentiation into cardiomyocytes, and examined the underlying mechanisms guiding the process.

Methods and Results: Classical hanging-drop method was used to induce human iPSC conversion into EBs, which were then treated with or without EleS for 1 to 30 days. We then examined the percentage of contracting EBs as well as the overall contraction rate. Surprisingly, we found that EBs started to beat spontaneously after only 2 days in the EleS group, much quicker than the cells without EleS, which took 7 days before onset of spontaneous beating. In addition, the percentage of contracting cardiac progenitor cells (CPCs) and the contraction rate were significantly higher in the EleS-treated group than in the non-EleS-treated group. Beating CPCs were confirmed using a genetically encoded calcium indicator (GCaMP5 driven by troponin T gene promoter) which consists of a Ca2+-sensitive calmodulin domain fused to GFP. Furthermore, expression of cardiac differentiation markers such as $\alpha$-sarcomeric actinin, CTnT, aMHC, GATA4 and NKX2.5 were markedly increased by EleS as compared to non-EleS, as examined by immunocytochemical analysis and real time PCR. Finally, we identified p38 MAP kinase signaling as one of the key regulators in the underlying mechanism by which EleS accelerates cardiac differentiation.

Conclusions: EleS enhances efficiency of cardiac differentiation in iPSCs and promotes maturation of these differentiated cardiomyocytes, directly implantation of these differentiated cardiomyocytes into myocardium after $\mathrm{MI}$ is a novel promising approach for clinical application of iPSCs.

No conflict of interest 
81

\title{
CELL THERAPY IN MYOCARDIAL PROTECTION \\ MESENCHYMAL STEM CELL SUBPOPULATIONS FOR INFARCT REPAIR
}

\author{
$\underline{B}_{\text {B. Dawn }}^{2}$, G. Cheng', A. Samantha², A. Labedz-Maslowska1 , Y.-T. Xuan², M. Girgis², E. Zuba- \\ Surma ${ }^{1}$ \\ ${ }^{1}$ Department of Cell Biology, Faculty of Biochemistry, Biophysics and Biotechnology, \\ Jagiellonian University, Krakow, Poland \\ ${ }^{2}$ Division of Cardiovascular Diseases, and the Midwest Stem Cell Therapy Center, \\ University of Kansas Medical Center, Kansas City, KA, USA
}

The outcomes of heart repair with bone marrow mesenchymal stem cells (MSCs) have been highly variable. Given the heterogeneous nature of mesenchymal cells, we hypothesized that subpopulations of MSCs would be more effective for infarct repair after myocardial infarction (MI). Unfractionated MSCs were obtained from mice using expansion culture, and subpopulations were derived using two-step FACS isolation. Our in vitro studies identified a Sca-1+/CD45-/c-kit/Thy1+/CD105- MSC population (CD105- MSCs) that exhibited mRNA expression profile favorable for cardiac repair. The CD105- MSCs also exhibited greater propensity toward cardiac differentiation. The efficacy of these cells in vivo was tested in a mouse model of reperfused $\mathrm{MI}$ with 30-min coronary occlusion followed by reperfusion. Intramyocardial injection of CD105MSCs was associated with superior preservation of LV function and improved remodeling. Our data suggest that these beneficial properties may be due to a primitive phenotype of CD105MSCs and their biological attributes. The use of this MSC subpopulation may potentially improve the outcomes of cell therapy in humans.

No conflict of interest 
84

ACUTE ISCHEMIA, ACUTE CORONARY SYNDROMES, ACUTE MYCARDIAL INFARCTION

TRANSCRIPTOME PROFILING BY RNA-SEQUENCING REVEALS DIFFERENTIALLY EXPRESSED ANNOTATED AND NOVEL GENES IN THE WHOLE BLOOD OF PATIENTS WITH STEMI VS. NSTEMI

G. Colombo ${ }^{1}$, M. Chiesa ${ }^{1}$, E. Bono ${ }^{1}$, V. Milazzo², G. Marenzi², L. Piacentini'

${ }^{1}$ IRCCS Centro Cardiologico Monzino, Unit of Immunology and Functional Genomics, Milano, Italy

2IRCCS Centro Cardiologico Monzino, Cardiac Intensive Care Unit, Milano, Italy

Aims: ST-segment elevation (STEMI) and non-ST-segment elevation myocardial infarction (NSTEMI) are respectively associated with a transmural event with an occlusive thrombus or a subendocardial occurrence with an incomplete/transient obstruction in the culprit coronary. Identification of specific differences in transcriptional profiles between STEMI and NSTEMI may provide crucial clues on their pathophysiology. We thus analyzed the whole-blood transcriptome in STEMI vs. NSTEMI patients through RNA-Sequencing.

Method: Total RNA was extracted from peripheral blood of 19 patients presenting with STEMI and 19 with NSTEMI, matched for age, sex, and cardiovascular risk factors. Poly(A)+ enriched libraries were prepared for paired-end sequencing. An empirical Bayesian estimation and exact test based on the negative binomial distribution were used to compare STEMI vs. NSTEMI transcriptomes. Gene-set enrichment analysis was used to infer biological processes distinguishing the two phenotypes.

Results: We identified 20073 genes, which included 5178 putative novel intergenic genes $>200$ nucleotides. Among the latter, $2.4 \%$ presented a high coding potential, being the others novel long non-coding RNAs. Analysis showed that 859 annotated and 115 novel genes were differentially expressed between STEMI and NSTEMI. Genes with higher expression in STEMI were associated with regulation of cytokine-mediated signaling pathways, MAPK-JNK pathways, autophagy, and apoptosis, whereas in NSTEMI with Toll-like Receptor cascades, helicase activity, DNA unwinding, and non-coding RNA processing.

Conclusion: RNA-Sequencing allowed identifying known and novel transcripts differentially regulated in STEMI vs. NSTEMI peripheral blood. Our analysis depicts a possible scenario underlying pathophysiological features of these two conditions, which could provide a framework for development of diagnostic biomarkers and therapeutic strategies.

No conflict of interest 
86

ACUTE ISCHEMIA, ACUTE CORONARY SYNDROMES, ACUTE MYCARDIAL INFARCTION

PRETREATMENT WITH ASPIRIN IN ACUTE CORONARY SYNDROMES: LESSONS FROM THE ACUITY AND HORIZONS-AMI TRIALS

S.J. Brener 1 , R. Mehran², A. Lansky3, G. Ayele ${ }^{4}$, G. Stone ${ }^{5}$

${ }^{1}$ NY Methodist Hospital, Cardiology, New York, USA

${ }^{2}$ Mount Sinai Medical Center, Cardiology, New York, USA

${ }^{3}$ Yale University Medical Center, Cardiology, New Haven, USA

${ }^{4}$ Cardiovascular Research Foundation, Biostatistics, New York, USA

${ }^{5}$ Columbia University Medical Center, Cardiology, New York, USA

Aims: Aspirin is promptly administered to patients presenting with acute coronary syndromes (ACS). It is not known whether aspirin pretreatment (PT) in ACS patients is beneficial, particularly since some, but not all prior studies identified aspirin PT as an independent risk factor for adverse ischemic events. We set to study the effect of aspirin PT in patients with ACS enrolled in two large randomized clinical trials.

Method: Patients enrolled in the ACUITY and HORIZONS-AMI trials were analyzed according to aspirin PT within 5-7 days before ACS. We evaluated the incidence of death, myocardial infarction (MI), target vessel revascularization (TVR), stent thrombosis and bleeding at 30 days and at 1 year. Multivariable regression analysis was performed for all-cause and cardiac death.

Results: Among 17,387 patients, 10,587 (60.9\%) were pretreated with aspirin. Pretreated patients were significantly older and more likely to have diabetes mellitus, hypertension and prior revascularization, and receive only medical therapy without revascularization. Aspirin PT was associated with reduced 30 -day death (adjusted $\mathrm{HR}=0.68$ [0.49-0.94], $\mathrm{P}=0.04$ ). MI was more frequent in the aspirin PT group at 30 days $(P<0.0001)$, while stent thrombosis was less frequent $(P=0.01)$. A strong interaction was present such that aspirin $P T$ was associated with reduced 30 day death in patients with non-ST-segment elevation ACS (NSTEACS), but not in those with STsegment elevation $\mathrm{MI}(\mathrm{P}=0.001)$.

Conclusion: Among patients with ACS in these 2 large prospective studies, aspirin PT identified a higher risk cohort and was an independent predictor of reduced mortality at 30 days, especially in patients with NSTEACS.

No conflict of interest 
87

ACUTE ISCHEMIA, ACUTE CORONARY SYNDROMES, ACUTE MYCARDIAL INFARCTION

THE ROLE OF ANGIOPLASTY IN THE MANAGEMENT OF ACUTE CORONARY SYNDROME IN PATIENTS WITH CHRONIC KIDNEY DISEASE

M. Zaman ${ }^{1}$, R.S. Gorantla', H. Uppal ${ }^{3}$, D. Lavu' ${ }^{3}$, S. Chandran 4 , R. Potluri ${ }^{3}$

${ }^{1}$ University Hospital of South Manchester, Cardiology, Manchester, United Kingdom

${ }^{2}$ Columbia University of Physicians and Surgeons- Bassett Medical Center, Internal Medicine, New York, USA

${ }^{3}$ Aston University, Acalm Study Unit in Colloboration in with Aston Medical School, Birmingham, United Kingdom

${ }^{4}$ North Western Deanery, Acute Medicine, Manchester, United Kingdom

Aims: In patients with significant chronic kidney disease (CKD) the evidence for angioplasty in acute coronary syndrome (ACS) is less well known. We sought to investigate if angioplasty affected mortality and outcomes in patients with CKD, presenting with ACS.

Method: Anonymous information on patients with ACS, attending a large multi-ethnic general hospital in Manchester, United Kingdom in the period 2000-2013 was obtained from the local health authority computerised hospital activity analysis register using ICD-10 and OPCS coding systems according to the ACALM protocol.

Results: Out of 25294 patients with ACS, 1612(6.4\%) patients has CKD stage 3 or worse. Of these, $87(5.4 \%)$ patients underwent angioplasty with mean age 66.6 years and were significantly younger than patients who did not undergo angioplasty. Patients who did not undergo angioplasty had higher prevalence of Ischaemic Stroke (7.1\% vs $1.1 \%)$, Heart Failure $(48.5 \%$ vs $27.6 \%)$ and carotid artery disease $(0.9 \%$ vs $0 \%)$ whilst angioplasty patients had higher prevalence of previous ACS(28.2\% vs $32.2 \%)$ and peripheral vascular disease(10.4\% vs $12.6 \%$ ). Multi-nominal logistic regression analysis revealed Heart Failure (RR2.15), Ischaemic Stroke (RR2.54) as significant predictors of mortality in this patient group. Angioplasty (RR4.42) and previous ACS (RR1.76) conferred mortality improvement.

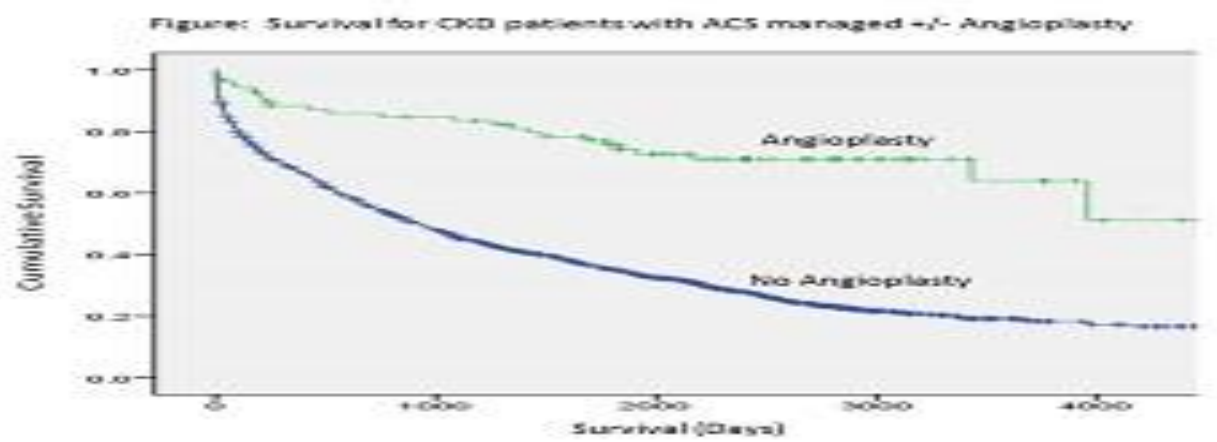


Conclusion: We have shown that angioplasty confers significantly improved mortality in the management of ACS in patients with CKD. Previous ACS also confers a mortality benefit, possibly reflecting previous angioplasty or medical therapy. The findings of this study highlight the need for clinicians to conscientiously think about the individual benefits and risks of angioplasty for every patient with CKD rather than confining to stereotypes.

No conflict of interest 
88

ACUTE ISCHEMIA, ACUTE CORONARY SYNDROMES, ACUTE MYCARDIAL INFARCTION

\title{
EFFECT OF INTRACORONARY NICARDIPINE ON THE SPONTANEOUS CORONARY SLOW FLOW PHENOMENON
}

\author{
M.P. Savage ${ }^{1}$, M. Morris ${ }^{1}$, J. Finley², D. Fischman ${ }^{1}$, N. Ruggiero ${ }^{1}$, P. Walinsky ${ }^{1}$, M. McCarey ${ }^{3}$, \\ S. Adams $^{3}$ \\ ${ }^{1}$ Thomas Jefferson University Hospital, Cardiology, Philadelphia, PA, USA \\ ${ }^{2}$ Aria Health, Cardiology, Philadelphia, PA, USA \\ ${ }^{3}$ Jefferson Clinical Research Institute, University, Philadelphia, PA, USA
}

\begin{abstract}
Aims: An under-recognized cause of chest pain, the coronary slow flow (CSF) phenomenon is characterized by delayed coronary opacification during diagnostic angiography in the absence of obstructive CAD. Because of its angiographic resemblance to no-reflow during $\mathrm{PCl}$ (a condition associated with microvascular spasm), we hypothesized that spontaneous CSF may be reversed by pharmacological intervention with intracoronary (IC) nicardipine.
\end{abstract}

Method: The effect of IC nicardipine on CSF was assessed in 26 consecutive patients. CSF was defined as spontaneously delayed flow (<TIMI 3 ) during diagnostic angiography in the absence of epicardial CAD or other conditions potentially associated with impaired flow. Nicardipine was administered directly via the coronary catheter as a $200 \mathrm{mcg}$ bolus after which repeat angiography was performed. Coronary flow before and after nicardipine was assessed by TIMI flow grade and corrected TIMI frame count (TFC) assessments.

Results: The study population consisted of 18 men and 8 women, aged $53 \pm 13$ years. The clinical presentation was rest angina in $19(73 \%)$ patients. At baseline, CSF with <TIMI 3 flow was observed in 46 vessels (24 LAD, 7 LCX, 15 RCA). TFC was prolonged (>27) in 61/78 (78\%) epicardial vessels. IC nicardipine characteristically produced markedly accelerated coronary filling; this observation was corroborated by TFC analysis. TFC was $54 \pm 13$ before versus $15 \pm 5$ after nicardipine $(p<0.001)$. All vessels demonstrated TIMI 3 flow and TFC $<28$ after nicardipine.

Conclusion: Intracoronary nicardipine appears highly effective in reversing spontaneous CSF and implicates microvascular spasm in its pathogenesis. Future studies of oral calcium channel blockers in the management of patients with CSF are needed.

No conflict of interest 


\section{0 \\ ACUTE ISCHEMIA, ACUTE CORONARY SYNDROMES, ACUTE MYCARDIAL INFARCTION DETECTION AND DYNAMICS OF VENTRICULAR THROMBUS BY CMR AFTER
REPERFUSED ST-SEGMENT ELEVATION MYOCARDIAL INFARCTION \\ C. Bonanad', E. Cambronero', R. Paolo' ${ }^{1}$, M.P. Lopez-Lereu², S. García-Blas ${ }^{1}$, M. Jose Vicente², J. Gavara', F.J. Chorro', V. Bodi' \\ ${ }^{1}$ Hospital Clínico de Valencia, Cardiology, Valencia, Spain \\ ${ }^{3}$ Hospital Clínico de Valencia-ERESA, Cardiology, Valencia, Spain}

Aims: Cardiac magnetic resonance (CMR) has emerged as the gold standard for establishing this diagnosis. We aimed to characterize the incidence, predictors and dynamics of this finding using CMR. The ability of echocardiography to identify CMR-derived VTh was also addressed.

Method: From 2004 to 2014 we prospectively enrolled 574 patients with a first STEMI treated with primary percutaneous intervention and studied with CMR 1 week after infarction. Of them, 391 (68\%) underwent a second CMR within the first 6 months post-infarction.

Results: In 1-week CMR, VTh was detected in 24 patients (4\%). Patients with VTh displayed a more depressed LVEF, more dilated LV volumes and LV mass and a larger \% of LV mass with edema, microvascular obstruction and necrosis $(p<0.001)$. Of 24 patients with VTh, $23(96 \%)$ had an anterior infarction and $21(87 \%)$ a depressed LVEF (as derived from validated cut-off values). VTh occurred in $1 / 143(0.7 \%)$ patient with non-anterior infarction and preserved LVEF, in $2 / 214(0.9 \%)$ with either anterior infarction or depressed LVEF and in 21/217 (10\%) with both anterior infarction and depressed LVEF ( $p<0.001$ for the trend). In the multivariate analysis, adjusted for baseline and CMR characteristics, the presence of simultaneous depressed LVEF and anterior infarction independently predicted the occurrence of VTh.

Conclusion: CMR allows for a comprehensive characterization of the incidence, predictors and dynamics of left ventricular thrombus after a first reperfused STEMI. This finding occurs in around $4 \%$ of patients and associates with the presence of severe left ventricular structural damage.

No conflict of interest 
91

\title{
ACUTE ISCHEMIA, ACUTE CORONARY SYNDROMES, ACUTE MYCARDIAL INFARCTION
}

\section{IMPACT OF OBESITY ON IN-HOSPITAL MORTALITY OF ST-ELEVATION MYOCARDIAL INFARCTION PATIENTS UNDERGOING PRIMARY PERCUTANEOUS CORONARY INTERVENTION}

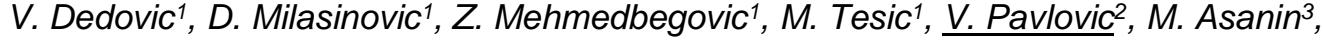 \\ V. Vukcevic ${ }^{1}$, V. Kanjuh ${ }^{4}$, G. Stankovic ${ }^{1}$ \\ ${ }^{1}$ Cardiology Clinic- Clinical Center of Serbia, Catheterisation Laboratory, Belgrade, Serbia \\ ${ }^{2}$ Cardiology Clinic- Clinical Center of Serbia, Cardiology, Belgrade, Serbia \\ ${ }^{3}$ Cardiology Clinic- Clinical Center of Serbia, Emergency Department, Belgrade, Serbia \\ ${ }^{4}$ Serbian Academy of Sciences and Arts, Board on cardiovascular pathology, Belgrade, Serbia
}

Aims: Recent studies on relationship between body mass index (BMI) and outcomes after primary percutaneous coronary intervention $(\mathrm{pPCl})$ showed conflicting results.

Method: We analyzed data records of 3626 consecutive patients who underwent $\mathrm{pPCl}$ in a high volume tertiary center from 2009 to er4. Patients were divided in tertiles according to BMI (nonoverweighed: $\mathrm{BMI}<25.1 \mathrm{~kg} / \mathrm{m} 2$; lite-overweighed: $\mathrm{BMI} \geq 25.2<28.6 \mathrm{~kg} / \mathrm{m} 2$ and obese: $\mathrm{BMI} \geq$ $28.6 \mathrm{~kg} / \mathrm{m} 2)$. Primary endpoint was in-hospital mortality.

Results: The overall intra-hospital mortality was $4.6 \%$. Obese patients were younger with higher conventional risk factors prevalence (Table 1). In-hospital mortality decreased with the degree of obesity and was the lowest in the highest BMI tertile (2.8\% vs. $5.9 \%$ vs. $6.7 \%$; $p<0.01)$. After adjusting for demographic and clinical factors, higher BMI remained independent predictor of lower in-hospital mortality (OR $0.75,95 \% \mathrm{Cl} 0.57-0.89, \mathrm{p}<0.01)$. Other independent factors included age (OR 1.06, 95\% Cl1.04-1.08, $\mathrm{p}<0.01$ ), diabetes mellitus (OR 2.72, 95\% Cl 1.83$3.43, \mathrm{p}<0.01$ ) and hyperlipoproteinemia (OR 2.07, 95\% Cl 1.45-2.90, $\mathrm{p}<0.01$ ).

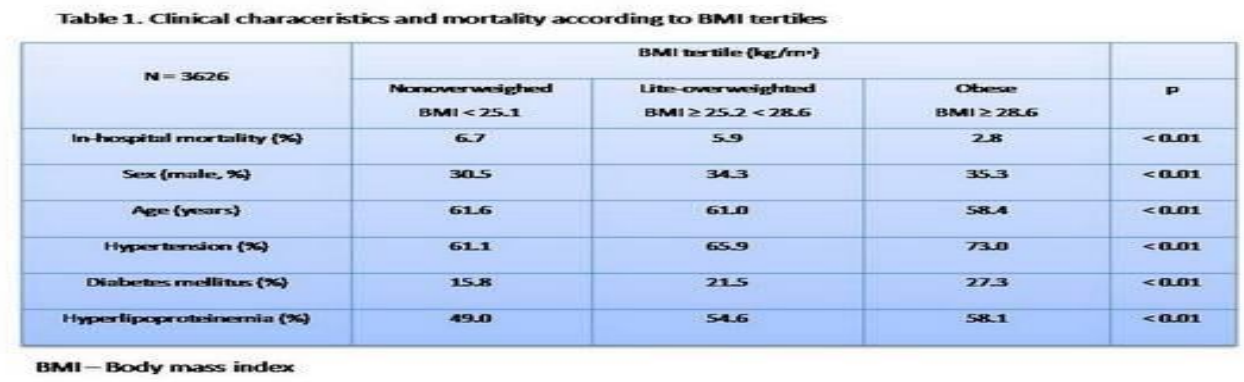

Conclusion: Obesity, expressed as BMI values higher than $28.6 \mathrm{~kg} / \mathrm{m} 2$, is a predictor of lower inhospital mortality in STEMI patients treated with $\mathrm{pPCl}$.

No conflict of interest 
97

ANGIOGENESIS, CELL THERAPY

\title{
SEEING STEM CELL BIOLOGY IN THE LIGHT OF PHYSICAL ENERGIES: MUTANT VIBRATIONS FOR REGENERATIVE MEDICINE
}

\section{Ventura}

Stem Wave Institute for Tissue Healing (SWITH), Gruppo Villa Maria (GVM) Care \& Research Ettore Sansavini Health Science Foundation, Lugo, Italy

\begin{abstract}
Aims: For decades Scientists have used chemical tools to affect cell behavior. Here, we investigated whether exposure to physical energies, including electromagnetic fields and sound vibrations, may affect (stem) cell fate and senescence patterning.
\end{abstract}

Methods: Mouse embryonic and human adipose-derived stem cells (hADSCs), or human skin fibroblasts were exposed to asymmetrically conveyed radio electric fields (ACREF) by the aid of a Radio Electric Asymmetric Conveyer (REAC). Gene and protein expression were monitored by real-time RT-PCR and Western blot analysis, respectively. Confocal microscopy and immunofluorescence analysis were used to assess (stem) cell commitment.

Results: Exposure to ACREF was able to transform mouse embryonic stem cells and hADSCs into cardiac myocytes, neurons, skeletal muscle, and endothelial cells. Moreover, ACREF acted as a sort of "time machine" even able to reprogram human adult non-stem somatic cells, like skin fibroblasts, into cell types in which these cells would never otherwise appear, including myocardial, neural, and skeletal muscle cells. Intriguingly, hADSC exposure to ACREF was found to counteract and reverse stem cell senescence in vitro, acting at the level of both telomerasedependent and -independent pathways.Consonant with these observations is our discovery that cells can produce acoustic vibrations, and express "vibrational" signatures of their health and differentiating potential.

Conclusions: The present data demonstrate that stem cell fate can be remarkably modulated by physical energies. This discovery prompts a deeper understanding of the interconnections between the physical universe and the living world in the attempt to further approach the information of Life.

Key Words: Stem Cells; Reprogramming; Senescence; Electromagnetic Fields; Sound Vibration No conflict of interest 
101

\title{
RISK FACTORS, DIABETES
}

\section{MICRORNA-X CAN IDENTIFY SMOKERS AT RISK FOR ATHEROSCLEROSIS}

\author{
M. de Ronde ${ }^{1}$, M. Kok ${ }^{1}$, E. Creemers ${ }^{2}$, P. Moerland ${ }^{3}$, A. Neele ${ }^{4}$, J. vd Bossche ${ }^{4}$, M. de Winther , \\ J. Meijers ${ }^{5}$, S.J. Pinto-Sietsma ${ }^{1}$ \\ ${ }^{1}$ Academic Medical Centre, Vascular Medicine, Amsterdam, Netherlands \\ ${ }^{2}$ Academic Medical Centre, Cardiology, Amsterdam, Netherlands \\ ${ }^{3}$ Academic Medical Centre, Clinical Epidemiology-Biostatistics and Bioinformatics, Amsterdam, \\ Netherlands \\ ${ }^{4}$ Academic Medical Centre, Biochemistry, Amsterdam, Netherlands \\ ${ }^{5}$ Sanquin, Plasma proteins, Amsterdam, Netherlands
}

\begin{abstract}
Aims: MicroRNAs (miRNAs) are small RNAs that control gene expression. They are involved in cardiovascular disease, which is influenced by smoking. We investigated if smoking influences miRNA expression.
\end{abstract}

Method: We investigated the effect of smoking on monocyte miRNAs using microarray in a cohort of 40 subjects with coronary artery disease (CAD) and 40 controls.

Results: We observed that only among smokers, one specific miRNA-X was highly expressed among some smokers whereas it was not among others. To confirm this observation, we repeated the analysis in a second microarray of 27 subjects with CAD and 40 of their healthy family members and found the same results. To investigate whether high miRNA-X levels among smokers were related to functional changes in monocytes, we measured monocyte surface markers in smokers $(n=20)$ and found that miRNA-X was positively correlated to atherosclerosis related monocyte surface markers CD206, CD29 and CD45RA. Subsequently, we transfected human macrophages with miRNA-X and found that CD206 was significantly upregulated, but CD29 and CD45RA were not. Since miRNA-X is high in some smokers which is accompanied by atherosclerotic monocyte surface marker changes, we questioned whether smokers with high miRNA-X are more prone to atherosclerosis. Among smokers with coronary calcifications $(n=38)$ we found higher miRNA-X levels as compared to smokers without $(n=32)(O R 2.4,95 \% \mathrm{Cl} 1.8$ 4.6, $\mathrm{P}=0.007$ ).

Conclusion: We show that high miRNA-X identifies subclinical atherosclerosis in some smoking individuals, possibly through increased CD206 on the monocyte. This could mean that some smokers might be prone to the adverse effects of smoking, while others are not.

No conflict of interest 
102

RISK FACTORS, DIABETES

\title{
STATIN UTILISATION AND LDL-CHOLESTEROL GOAL ATTAINMENT IN VERY HIGH CARDIOVASCULAR RISK PATIENTS: INSIGHTS FROM AN ITALIAN GENERAL PRACTICE POPULATION
}

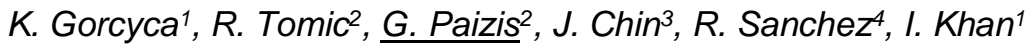 \\ ${ }^{1}$ Sanofi, Global Health Economics and Outcomes Research, Bridgewater- NJ, USA \\ ${ }^{2}$ Sanofi, Value \& Patient Access, Milan, Italy \\ 3IMS Health, Real World Evidence Solutions, Jersey City- NJ, USA \\ ${ }^{4}$ Regeneron Pharmaceuticals- Inc., Health Economics and Outcomes Research, Tarrytown- NY, \\ USA
}

Aims: ESC/EAS cholesterol guidelines recommend reducing low-density lipoprotein cholesterol (LDL-C) to $<1.8 \mathrm{mmol} / \mathrm{L}(70 \mathrm{mg} / \mathrm{dL})$ for very high cardiovascular risk patients. We summarise lipidmodifying therapy (LMT) and achieved LDL-C levels in patients with documented cardiovascular disease and/or diabetes from an Italian general practice cohort.

Method: This analysis included patients from the Cegedim general practice database in Italy meeting the following criteria: LDL-C measurement in 2013 (index date); age $\geq 20$ years; continuous representation in database $\geq 2$ years; and $\geq 1$ very high cardiovascular risk condition. Patients were grouped into the following hierarchical, mutually-exclusive categories: acute coronary syndrome within 12 months; other coronary heart disease; ischaemic stroke; peripheral arterial disease; and diabetes mellitus. Patients were considered treated with LMT if covered by a prescription within 30 days prior to index date.

Results: 46412 patients met the inclusion criteria. Median age was 71 years, $54 \%$ were male, and mean LDL-C was $2.7 \mathrm{mmol} / \mathrm{L}(104 \mathrm{mg} / \mathrm{dL})$. Overall, $56 \%$ of patients were receiving statins; of these, $13 \%$ were receiving high-intensity statins. $42 \%$ were not treated with LMT. Achievement of LDL-C $<1.8 \mathrm{mmol} / \mathrm{L}(70 \mathrm{mg} / \mathrm{dL})$ was only $16 \%$, with a possible relationship to LMT intensity. 


\begin{tabular}{|c|c|c|c|c|c|c|}
\hline \multicolumn{7}{|c|}{ Table 1. LMT use by hierarchical classification } \\
\hline & $\begin{array}{c}\text { Recent acule } \\
\text { coronary } \\
\text { syndrome } \\
\mathrm{N}=690\end{array}$ & $\begin{array}{c}\text { Other } \\
\text { coronary } \\
\text { heart } \\
\text { disease } \\
\mathrm{N}=15362\end{array}$ & $\begin{array}{c}\text { Ischaemic } \\
\text { stroke } \\
\mathrm{N}=4131\end{array}$ & $\begin{array}{c}\text { Peripheral } \\
\text { arterial } \\
\text { disease } \\
\mathrm{N}=4163\end{array}$ & $\begin{array}{c}\text { Diabetes } \\
\text { mellitus } \\
\mathrm{N}=22066\end{array}$ & $\begin{array}{c}\text { Total } \\
N=46412\end{array}$ \\
\hline $\begin{array}{l}\text { High-intensity } \\
\text { statin }\end{array}$ & $43 \%$ & $14 \%$ & $5 \%$ & $5 \%$ & $3 \%$ & $7 \%$ \\
\hline $\begin{array}{l}\text { Low-to-moderate } \\
\text { intensily statin }\end{array}$ & $41 \%$ & $54 \%$ & $55 \%$ & $48 \%$ & $43 \%$ & $48 \%$ \\
\hline $\begin{array}{l}\text { Non-statin LMT } \\
\text { only }\end{array}$ & $3 \%$ & $2 \%$ & $2 \%$ & $2 \%$ & $3 \%$ & $2 \%$ \\
\hline No current LMT & $14 \%$ & $30 \%$ & $38 \%$ & $46 \%$ & $51 \%$ & $42 \%$ \\
\hline \multicolumn{7}{|c|}{$\begin{array}{l}\text { Statins include mono and combination therapies; high-intensity statin defined as atovastatin } 40 \text { and } 80 \mathrm{mg} \text { and } \\
\text { rosuvastatin } 20 \text { and } 40 \mathrm{mg}\end{array}$} \\
\hline
\end{tabular}

Conclusion: In a cohort of very high cardiovascular risk patients, overall statin use and LDL-C goal attainment was suboptimal. Strategies to increase the appropriate use of LMTs are needed to further reduce risk due to elevated LDL-C.

Conflict of Interest Disclosure Statement: Employee of Sanofi 
103

RISK FACTORS, DIABETES

\section{POSTMORTEM HEART WEIGHT: RELATION TO BODY SIZE AND EFFECTS OF CARDIOVASCULAR DISEASE AND CANCER}

N.T. Kumar ${ }^{1}$, K. Liestø/, E.M. Løberg ${ }^{1}$, H.M. Reims ${ }^{3}$, J. Mæhlen ${ }^{1}$

${ }^{1}$ Oslo University Hospital-Ullevål, Department of Pathology, Oslo, Norway

${ }^{2}$ University of Oslo, Department of Informatics, Oslo, Norway

${ }^{3}$ Oslo University Hospital-Rikshospitalet, Department of Pathology, Oslo, Norway

Aims: We aimed to describe the relationship between body size and heart weight in a large autopsy cohort, and to compare heart weight in patients with cancer, CVD, and other diseases.

Method: Registered information, including cause of death, evidence of cancer and/or CVD, heart weight, body weight, and height was extracted from the autopsy reports of 1410 men and women. The study population was divided in groups according to cause of death; cancer, CVD, mixed group who died from cancer and CVD and/or lung disease, and a reference group with patients who did not die from any of these conditions.

Results: In this last group, heart weight correlated only slightly better with BSA than body weight, and nomograms based on body weight were presented. Compared to the reference group, heart weight was significantly lower in cancer patients and higher in patients who died from CVD. Similar results were obtained in linear regression models adjusted for body weight and age. Among CVD, heart valve disease had the greatest impact on heart weight, followed by old myocardial infarction, coronary atherosclerosis, and hypertension. Absolute heart weight decreased with age, but we demonstrated an increase relative to body weight.

Conclusion: The weight of the human heart is influenced by various disease processes, in addition to body weight, gender, and age. While the most prevalent types of CVD are associated with increased heart weight, patients who die from cancer have lower average heart weight than other patient groups. 

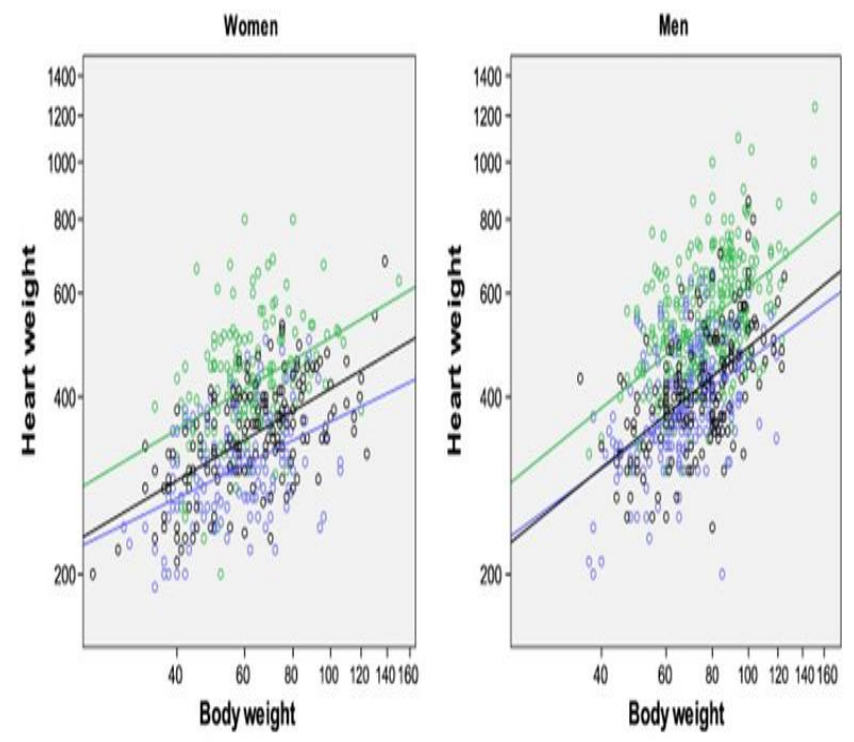

Fig. 2. Scatter plots of heart weight ( $g$ ) and body weight (kg) in women and men. o (black): reference group; o (green); death by CVD; o (blue): death by cancer. Fit line in corresponding color. Scale is logarithmic.

\section{No conflict of interest}




\title{
RISK FACTORS, DIABETES
}

\section{WEIGHT LOSS ASSOCIATED WITH SOCIOECONOMIC STATUS IN PATIENTS WITH ANGINA PECTORIS, 5 YEARS AFTER AN ANGIOGRAPHY}

\author{
A. Tchicaya ${ }^{1}$, N. Lorentz ${ }^{1}$, J. Beissel${ }^{2}$, D. Wagner ${ }^{2}$, S. Demarest ${ }^{3}$, M. Baumann ${ }^{4}$ \\ ${ }^{1}$ Luxembourg Institute of Socio-Economic Research, Living Conditions, Esch-sur-Alzette, \\ Luxembourg \\ ${ }^{2}$ National Institute of Cardiac Surgery and Interventional Cardiology INCCI, INCCI, Luxembourg- \\ ville, Luxembourg \\ ${ }^{3}$ Institute of Public Health, Public Health and Surveillance, Brussels, Belgium \\ ${ }^{4}$ University of Luxembourg, Research Unit INSIDE- Institute Health \& Behaviour, Esch-sur- \\ Alzette, Luxembourg
}

Aims: The aim of the study is to assess the association between weight loss and socioeconomic status (SES) in patients with cardiovascular disease (CVD) such as angina pectoris.

Method: In 2013-2014, 1,873 patients out of 4,391 that had undergone an angiography in 20082009 participated in a follow-up study. Among these patients, 457 suffered from angina pectoris and were obese or overweight at baseline. A self-administered questionnaire was completed. Moderate weight loss was defined as the loss of $5 \%-10 \%$, severe weigh loss as more than $10 \%$ of baseline weight. Household-income was used as proxy for SES. Logistic regression was applied to assess the association between weight loss and SES.

Results: During the reference period, $28 \%$ of the participants lost weight. A moderate weight loss was higher in patients with a household income between $3000 €$ and $4500 €$ per month (OR: 2.3; Cl 95\%: 1.0-5.2) and higher than $4500 €$ per month (OR: 3.0; Cl 95\%: 1.3-7.2) compared to the lowest income group (less than $3000 €$ per month). A severe weight loss was not associated with income. However, a severe weight loss was higher for retired patients (OR: 4.3; Cl 95\%: 1.117.0) compared to non-retired.

Conclusion: The potential reduction of health inequality by an elimination of social inequalities in weight loss among patients with angina pectoris might be substantial. Moderate weight loss in overweight patients with angina pectoris at baseline could be associated with household income. Intervention strategies in secondary prevention should address these social inequalities in order to support equity in health.

No conflict of interest 
105

\title{
RISK FACTORS, DIABETES
}

\section{COMPARISON OF THE HOSPITAL ARRIVAL TIME AND DIFFERENCES IN PAIN QUALITY BETWEEN DIABETIC AND NON-DIABETIC STEMI PATIENTS}

\author{
M. Gradiser ${ }^{1}$, I. Bilic ${ }^{2}$, J. Cmrecnjak ${ }^{3}$ \\ ${ }^{1}$ County Hospital Cakovec, Department of Endocrinology and Diabetes, Cakovec, Croatia \\ ${ }^{2}$ Faculty of Medicine Josip Juraj Strossmayer University of Osijek, \\ Department of Endocrinology and Diabetes, Osijek, Croatia \\ ${ }^{3}$ County Hospital Cakovec, Department of Cardiology, Cakovec, Croatia
}

\begin{abstract}
Aims: The aim of the study was to determine whether diabetic ST segment elevation myocardial infarction patients arrive in the emergency room later than non-diabetics, compare the differences in pain quality and quantity between those groups, and measure differences in the outcome after an index hospitalization.
\end{abstract}

Method: A total of 266 patients with first presentation of STEMI were included in our study during a period of two years, 62 with diabetes and 204 without diabetes type 2. Pain intensity and quality at admission were measured using a McGill short form questionnaire.

Results: Diabetic patients did not arrive significantly later than non-diabetic $\left(x^{2} ; p=0,105\right)$. Most diabetic patients described their pain as «slight» or «none» $\left(X^{2} ; p \leq 0,01\right)$, while most non-diabetic patients graded their pain as «moderate» or «severe» $\left(X^{2}, p \leq 0,01\right)$. The quality of pain tended to be more distinct in non-diabetic patients, while diabetic patients reported mainly shortness of breath $\left(X^{2}, p \leq 0,01\right)$. Diabetic patients were more likely to suffer a multi-vessel disease $\left(X^{2} ; p \leq\right.$ $0,01)$, especially in the late arrival group.

Conclusion: Therefore, cautious evaluation of diabetic patients and adequate education of target population could improve overall survival while well-organized care like a primary PCI Network program could significantly reduce CV mortality.

No conflict of interest 
106

RISK FACTORS, DIABETES

COMPARISON OF THE RATES OF CIGARETTE SMOKING CESSATION IN MIDDLE EASTERN PATIENTS FOLLOWING PCI IN TWO STUDIES 12 YEARS APART

\author{
A. Mmoudeh ${ }^{1}$, R. Tabbalat ${ }^{1}$, R. Hammoudeh ${ }^{2}$ \\ ${ }^{1}$ Istishari Hospital, Cardiology, Amman, Jordan \\ 2 Jordan University of Science and Technology School of Medicine, Medical Education, Irbid, \\ Jordan
}

Aims: We sought to study the rate of cigarette smoking cessation CSC among Jordanians following percutaneous coronary intervention $(\mathrm{PCl})$ who were enrolled in a 2 studies 12 years apart.

Method: The JoPCR1 registry enrolled 2426 patients who were followed for one year following $\mathrm{PCl}$. CS cessation (CSC) was assessed 12 months after $\mathrm{PCl}$ (group 1) and was compared with that in a study conducted 12 years after JoPCR1 (group 2).

Results: Of the 2426 patients enrolled in JoPCR1; 1055 (43.5\%) were CS (group 1). The rate of SCS after 12 months was $31 \%$. Compared with non-quitters, those who quit CS were more likely to be diabetics ( $50.4 \%$ vs. $42.0 \%$; $p=0.028)$, and to have low ejection fraction $(18.1 \%$ vs. $9.3 \%$; $\mathrm{p}=0.0004)$, and heart failure $(15.4 \%$ vs. $9.0 \% ; \mathrm{p}=0.0008)$. They were less likely to have past history of CAD (23.3\% vs. $34.0 \%$; $p=0.001)$ or previous $\mathrm{PCl}(17.3 \%$ vs. $24.0 \%$; $p=0.037)$. There were no differences in mean age, gender, BMI, type of ACS, or severity of CAD. Major cardiovascular events, during hospitalizations up to 1 year after $\mathrm{PCI}$ were similar in quitters and non-quitters, including cardiovascular death $(1.2 \%$ vs. $2.0 \% ; p=0.538)$, stent thrombosis $(3.1 \%$ vs. $2.5 \% ; p=0.788)$, and readmission for ACS (7.3\% vs. $5.9 \% ; p=0.535)$. CSC among group 2 $(\mathrm{n}=397)$ was $23 \%$, overall and $31 \%$ among those who had $\mathrm{PCl}$.

Conclusion: About 1 of 3 CS quit smoking after PCI. This rate was unchanged in 2 studies 12 years apart. Further studies are needed to identify more effective strategies to encourage CSC as a secondary preventive strategy.

No conflict of interest 
107

RISK FACTORS, DIABETES

\title{
SERUM PHOSPHATE PREDICTION OF INTERVAL CHANGES IN CARDIOVASCULAR RISK SCORES IN STABLE RENAL TRANSPLANT RECIPIENTS
}

\author{
K. Jillian', H. Mansel', M. Moser ${ }^{3}$, A. Hassan 3 , R. Mainra ${ }^{3}$, E. Hamdi ${ }^{3}$, A. Shoker ${ }^{3}$ \\ ${ }^{1}$ University of Saskatchewan, College of Medicine, Saskatchewan, Canada \\ ${ }^{2}$ University of Saskatchewan, College of Pharmacy and Nutrition, Saskatchewan, Canada \\ ${ }^{3}$ University of Saskatchewan, Department of Medicine, Saskatchewan, Canada
}

\begin{abstract}
Aims: We calculated rate of changes in The Cardiovascular Risk Calculator for Renal Transplant Recipients (CRCRTR MACE) in clinically stable renal transplant recipients (RTR) to identify co-variables that associate with fast cardiovascular (CV)-risk progression.
\end{abstract}

Method: CRCRTR-MACE scores were calculated on 139 patients in 2011 and 2014. Score changes above and below median change in scores were labelled fast or slow CV-riskprogression. Multivariate analysis (MVA) was performed to identify variables significant to percent changes in scores. Receiver operating characteristic (ROC) analysis was performed, to define sensitivity and specificity of factors significant to fast score progression. Risk factors and serum inflammatory markers associations were tested $n$ a MVA model in a random sample of 86 patients to explain mechanism(s) of fast CV-risk progression.

Results: Follow up was 2.61(2.02 - 4.47) years. Slow and fast progression was present in $50.4 \%$ and $49.6 \%$ of patients, with a median change of $25.8 \%$ (-92.10/0- $1444.7 \%)$. MVA showed o/o changes in age and serum phosphate were the only significant variables impacting fast progression in scores. ROC showed 2011 serum phosphate of $1.15 \mathrm{mmolfL}$ to predict fast progression (area under the curve (AUC) of $0.628, P>0.0126$ ). Age above 4Syears combined with 2011serum phosphate above $1.15 \mathrm{mmol} / \mathrm{L}$ had a significantAUC of0.781 ( $\mathrm{P}<0.0010 \mathrm{IL}-1 \mathrm{a}$ and IL$28 \mathrm{~A}$ were significant associates with serum phosphate above $1.1 \mathrm{mmolfL}$ in the MVA.

Conclusion: Changes in CV-risk in RTR over time are highly variable. Serum phosphate, even in upper normal level predict worsening of CVD risk scores in stable RTR

No conflict of interest 
116

\author{
PRE AND POST CONDITIONING - SAVES THE HEART? \\ MECHANISMS OF REMOTE PRECONDITIONING \\ P. Kleinbongard \\ Institute for Pathophysiology, West German Heart and Vascular Center, \\ University of Essen Medical School, Germany
}

\begin{abstract}
Aims: In remote ischemic conditioning (RIC), brief and fully reversible episodes of ischemia/reperfusion in one vascular bed, tissue or organ confer a global protective phenotype and render remote tissues and organs resistant to ischemia/reperfusion injury. This phenomenon has been confirmed in different species, including humans. Cardioprotection by RIC has been documented as reduced infarct size in experimental animals.
\end{abstract}

Method: Patients undergoing elective or primary percutaneous coronary interventions or coronary artery bypass grafting had reduced biomarker release or reduced infarct size on imaging when undergoing RIC before the intervention.

Results: The signal transduction of RIC is not yet clear in detail. The peripheral stimulus can be chemical, mechanical or electrical in nature and appears to involve the activation of peripheral sensory nerves. The transfer of the protective signal from the periphery to the heart or other organs is through neuronal and humoral communications. Protection can be transferred, even across species, with plasma or a plasma-derived dialysate and involves nitric oxide, stromalderived factor-1 $\alpha$, microRNA-144, but also other, not yet identified factors. The signal transduction within the myocardium involves adenosine, bradykinin, cytokines and chemokines and the activation of their specific receptors. The RISK- and SAFE-kinase programs are activated by RIC. In human left ventricles with prior RIC, STAT 5 phosphorylation is increased, and mitochondrial function is improved.

Conclusion: Further and more comprehensive understanding of the molecular mechanisms underlying RIC will help overcome confounders and facilitate translation to patient care. For review see: Br J Pharmacol 172, 2015, 2010-25; JACC 65, 2015, 177-95 and Circ Res 116, 2015, 674-99.

No conflict of interest 
118

\author{
PRE AND POST CONDITIONING - SAVES THE HEART? \\ GENE EXPRESSION DYNAMICS DURING PRECONDITIONING \\ M. Gyöngyösi \\ Medizinische Universität Wien, Univ. Klinik für Innere Medizin II, Abteilung für Kardiologie \\ Vienna, Austria
}

\begin{abstract}
Aims: Ischemic preconditioning (IPC) is one of the most powerful protective mechanisms of the heart against sustained ischemia. The second window of protection (SWOP) depends strongly on the protein de novo synthesis, but the effectors and transcriptoms remain largely unknown. Therefore we have investigated the SWOP-induced gene expression in spatial- and timedependent modes.
\end{abstract}

Method: Domestic pigs underwent closed-chest ischemia-reperfusion injury, without subsequent lethal ischemia. After $5 \mathrm{~h}$ and $24 \mathrm{~h}$, the animal underwent euthanasia and gene expression profiles of different myocardial sites were performed using next generation sequencing.

Results: The sole IPC stimulus (without the masking effect of acute myocardial infarction) induced different time- and spatial-dependent gene regulation response. This was more pronounced at $5 \mathrm{~h}$ compared to $24 \mathrm{~h}$ post-IPC-stimulus, suggesting the importance of early activation of protein de novo synthesis in SWOP. SWOP activated survival-responsible and metabolic pathways.

Conclusion: The increased expression of numerous genes ascertained new mediators/effectors in IPC-induced cardioprotection, providing targets for pharmaceutical search of cardioprotective drugs.

No conflict of interest 


\section{1}

\section{PRE AND POST CONDITIONING - SAVES THE HEART? \\ MECHANISMS OF CARDIOPROTECTION OF SUBCELLULAR DEFECTS IN ISCHEMIC REPERFUSION INJURY}

N.S. Dhalla

Institute of Cardiovascular Sciences, St. Boniface Hospital Research Centre, Faculty of Health Sciences, University of Manitoba, Winnipeg, Canada

Aims: Earlier studies from our laboratory have demonstrated that oxidative stress plays a critical role in the development of intracellular $\mathrm{Ca}^{2+}$-overload, defects in subcellular organelles such as sarcolemma (SL) and sarcoplasmic reticulum (SR), as well as protease activation and cardiac dysfunction due to ischemia-reperfusion (I/R) injury. Since antioxidants such as N-acetylcysteine (NAC) and M-mercaptopropionylglycine (MPG) are known to attenuate I/R-induced cardiac dysfunction, this study was undertaken to examine their effects on I/R-induced changes in $\mathrm{Ca}^{2+}$ handling by cardiomyocytes as well as SL and SR activities.

Method: For this purpose, rat hearts perfused with and without NAC and MPG were subjected to $30 \mathrm{~min}$ of global ischemia followed by $30 \mathrm{~min}$ of reperfusion.

Results: Isolated cardiomyocytes from I/R hearts showed a marked increase in basal [Ca2+]i as well as low $\mathrm{Na}$ +-induced increase in [Ca2+]i; these $\mathrm{l} / \mathrm{R}$-induced changes in [Ca2+]i were attenuated by treatment of hearts with NAC and MPG. The depressed activities of SL Na+-K+ ATPase and $\mathrm{Na}-\mathrm{Ca} 2+$ exchanger, as well as $\mathrm{SR} \mathrm{Ca2+-uptake} \mathrm{and} \mathrm{Ca} 2+-$ release in $\mathrm{I} / \mathrm{R}$ hearts were also prevented by NAC and MPG. The I/R-induced increases in proteolytic enzyme activities and different parameters for oxidative stress were significantly reduced by NAC and MPG treatments. Perfusion of the hearts with oxyradical generating mixture or $\mathrm{H} 2 \mathrm{O} 2$, an oxidant, produced actions similar to those seen with $\mathrm{I} / \mathrm{R}$.

Conclusion: The results indicate that antioxidants improve cardiac function in $\mathrm{I} / \mathrm{R}$ hearts by attenuating the l/R-induced subcellular defects, protease activation and occurrence of intracellular $\mathrm{Ca} 2+-$ overload in cardiomyocytes.

No conflict of interest 
124

\section{DRUG THERAPY, CLINICAL ASPECTS}

ORAL NICORANDIL REDUCES ISCHEMIC ATTACKS IN PATIENTS WITH STABLE ANGINA: A PROSPECTIVE, MULTICENTER, OPEN-LABEL, RANDOMIZED, CONTROLLED STUDY AIMS

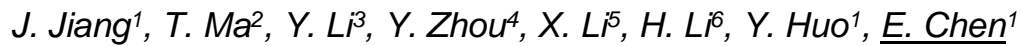

${ }^{1}$ Peking University First Hospital, Cardiovascular Department, Beijing, China

${ }^{2}$ Merck Serono Co.- Ltd, Medical Affairs, Beijing, China

3305 Military Hospital, Cardiology, Beijing, China

${ }^{4}$ Guangdong Provincial People's Hospital, Cardiology, Guangzhou, China

${ }^{5}$ Harbin Medical University Fourth Affiliated Hospital, Cardiology, Harbin, China

${ }^{6}$ Daqing Oilfield General Hospital- Daqing, Cardiology, Daqing, China

Aims: To evaluate the efficacy and safety of oral nicorandil in coronary heart disease (CHD) patients with stable angina.

Method: Eligible patients were randomized 1:1 to the nicorandil or control group. Current standard antianginal treatment was continued in both groups, while the patients in the nicorandil group received an additional 12 -week treatment of nicorandil ( $5 \mathrm{mg}$ thrice daily). Primary endpoint was the number of myocardial ischemia measured by $24 \mathrm{~h}$ Holter after 12 weeks treatment. Secondary endpoints included various $24 \mathrm{~h}$ Holter indicators, angina occurrence, 6-minute walking test (6MWT), ECG QT dispersion (QTd), safety and compliance. Primary analysis was performed using Poisson regression test and secondary analysis was performed using t-test or rank test. Clinical trial information: NCT01396395.

Results: Totally 402 patients were enrolled. Baseline characteristics were well balanced between the two groups. The number of myocardial ischemia attacks after treatment was significantly lower in the nicorandil group (LSMEANS 0.90) than the control group (LSMEANS 1.78), with an adjusted ratio of $0.50(95 \% \mathrm{Cl}: 0.30,0.84 ; \mathrm{P}=0.0086)$. No differences in total myocardial ischemic burden, maximum ST-depression, longest duration of ST-depression, 6MWT, or heart rate variability were noted between the two groups. Twenty three (11.7\%) and $13(6.3 \%)$ subjects reported at least one AE, respectively. The most common treatment-emergent AEs were chest pain, headache, and nausea. Mean compliance rate was $96.81 \%$ in the nicorandil group.

Conclusion: Nicorandil significantly reduced the number of ischemic attacks when added to standard antianginal treatment in CHD patients with stable angina. It was well tolerated with no new safety signal identified.

No conflict of interest 


\title{
DRUG THERAPY, CLINICAL ASPECTS
}

\section{ACETYLSALICYLIC ACID HYPERSENSITIVITY IN PATIENTS WITH CORONARY ARTERY DISESASE: DESENSITIZATION PROTOCOLS AND ALTERNATIVE THERAPIES}

\author{
G. Fuertes Ferre ${ }^{1}$, S. Laita ${ }^{2}$, G. Galache ${ }^{1}$, E. Sanchez Insa', J. Sánchez Rubio ${ }^{1}$, M.D.R. Ortas ${ }^{1}$, \\ J.A. Diarte ${ }^{1}$, I. Calvo 2 \\ ${ }^{1}$ Instituto Aragonés de Ciencias de la Salud - Hospital Universitario Miguel Serve, \\ Interventional Cardiology Unit, Zaragoza, Spain \\ 2Instituto Aragonés de Ciencias de la Salud - Hospital Universitario Miguel Serve, \\ Cardiology Department, Zaragoza, Spain
}

\begin{abstract}
Aims: Acetylsalicylic acid (ASA) represents the gold standard of antiplatelet therapy in coronary artery disease (CAD). Hypersensitivity to ASA limits treatment options. ASA desensitization (DS) protocols and alternative antiplatelet agents (AA) have been described. However, they are all based in non randomized trials that include small populations. It is important to know the safety and efficay of ASA DS and other AA in patients with CAD.
\end{abstract}

Method: We describe an overview of the latest literature and report our experience of the patients with ASA hypersensitivity and CAD in our center.

Results: In 10 recent studies, a total of 230 patients with history of ASA hypersensitivity and CAD were enrolled, including those presenting acute coronary syndrome and the cases treated with stents. ASA desensitisation success rate was $85-92 \%$. The incidence of major adverse cardiac events was low (only 3 patients). In our center 76 patients with history of ASA hypersensitivity and CAD (29 undergoing PCl) were treated with antiplatelet agents other than ASA, some of them following a DS protocol. We report our 8-year follow-up data.

Conclusion: ASA desensitization appears to be safe and effective in patients with CAD, including in those undergoing $\mathrm{PCl}$. When double antiplatelet treatment is needed, the use of alternative AA (Triflusal) does not translate into a higher rate of major adverse cardiac events.

No conflict of interest 
126

DRUG THERAPY, CLINICAL ASPECTS

THERAPEUTIC MONITORING OF DIRECT ORAL ANTICOAGULANTS: PRINCIPLES AND TEST INTERPRETATION

\section{H. Mani}

Goethe University Medical School, Internal Medicine, Frankfurt am Main, Germany

Aims: The introduction of direct oral anticoagulants (DOA) in stroke prevention, prophylaxis and long-term therapy of venous thromboembolism has overcome the limitations of vitamin $\mathrm{K}$ antagonists' treatment, and routinely performed drug monitoring may not required anymore. However, several scenarios, such as life-threatening bleeding or ischemic stroke during treatment, or the use of newly developed specific antidotes for DOA lead to the opinion that determination of the anticoagulant effects of DOA is definitely useful.

The objective of this review was to summarize current methods regarding measurement of the anticoagulant activity of DOA.

Method: Search in PubMed for studies that reported a relationship between drug levels and coagulation assay results.

Results: For dabigatran, a normal thrombin time excludes clinically relevant drug concentrations. Dilute thrombin time and ecarin-based assays show excellent linearity across drug concentrations and should be used for drug quantification.

For direct anti-Xa antagonists as rivaroxaban anti-Xa activity is linear over a wide range of drug levels. The Prothrombin Time (PT) in plasma is less sensitive for detecting high concentration of anti-Xa antagonists; a normal PT in plasma may not exclude clinically relevant levels, whereas PT measured in whole blood by point-of-care devices might be useful for valid diagnostic information in patients with suspected clinically relevant drug concentrations.

Conclusion: If threshold values of drug concentrations and the degree of an exact cutoff that defines a clearly increased hemorrhagic risk at trough levels for all DOA is defined in near future, therapeutic drug monitoring for DOA is effective in health outcomes and patients' quality of life.

No conflict of interest 
127

\title{
DRUG THERAPY, CLINICAL ASPECTS
}

\section{A SYSTEMATIC DIAGNOSTIC AND THERAPEUTIC APPROACH FOR THE TREATMENT OF PATIENTS AFTER CARDIO-PULMONARY RESUSCITATION: A PROSPECTIVE EVALUATION OF 212 PATIENTS DURING 5 YEARS}

\author{
V. Stefan ${ }^{1}$, K. Chakraborty' ${ }^{1}$ J. Hemker ${ }^{1}$, D. Vagts ${ }^{2}$, U. Hink ${ }^{3}$, T. Münzel' ${ }^{4}$, H. von Korn ${ }^{1}$ \\ ${ }^{1}$ Krankenhaus Hetzelstift, Cardiology, Neustadt an der Weinstrasse, Germany \\ ${ }^{2}$ Krankenhaus Hetzelstift, Anesthesiology and Intensive Care, Neustadt an der Weinstrasse, \\ Germany \\ ${ }^{3}$ Klinikum Frankfurt Höchst, Cardiology, Frankfurt, Germany \\ ${ }^{4}$ Universitätsmedizin Mainz, Cardiology, Mainz, Germany
}

\begin{abstract}
Aims: There is a need for a systematic treatment protocol for patients after resuscitation.
Method: A systematic diagnostic approach including ECG, echocardiography, urgent cardiac catheterization ("STEMI-like" workflow), pulmonary angiography, CT scan, pre-defined laboratory findings, IABP, hypothermia and cMRI was evaluated in our cardiology department prospectively over 5 years. The primary endpoints were survival and the Cerebral Perfomance Category Scale (CPCS).
\end{abstract}

Results: 212 patients were included into our protocol, mean age was 66.7 years, $71.2 \%$ were male. Ventricular fibrillation (VF) was observed in $46.7 \%$ of the cases. A significant coronary artery stenosis was found in $n=130$ patients and a percutaneous coronary intervention (PCI) was performed in 101 patients. An acute coronary syndrome (ACS) was diagnosed in 100 patients, 91 patients had a cardiomyopathy, and 7 patients had evidence for a Tako-Tsubo cardiomyopathy. Rare diagnoses were pulmonary embolism, long QT syndrome, early repolarization syndrome, hypertrophic cardiomyopathy and aortic dissection. An extracardiac cause was observed in 12 patients.

The survival rate was $35.9 \%$, a CPCS of $1 / 2$ was established in 67 patients. In patients being treated with a $\mathrm{PCI}$, a significant difference in mortality was found for TIMI flow $2 / 3$ vs $0 / 1(65.4 \%$ vs. $95.7, p<0.05)$. The was no statistically significant difference in mortality with respect to the use of intra-aortic balloon pumping $(70.0 \%$ vs. $63.6 \%, p=0.6)$. Hypothermia reduced mortality significantly $(52.7 \%$ vs $68.2 \%, p<0.05)$.

Conclusion: A systematic diagnostic and therapeutic algorithm using a "STEMI-like" workflow is feasible, safe and can improve prognosis.

No conflict of interest 
128

\title{
DRUG THERAPY, CLINICAL ASPECTS
}

\section{PSYCHOMETRIC PROPERTIES OF THE ARABIC MACNEW HEART DISEASE HEALTH- RELATED QUALITY OF LIFE QUESTIONNAIRE}

\author{
H. Rawas ${ }^{1}$, P. Yates ${ }^{2}$, K. Theobald ${ }^{2}$, R. Clark ${ }^{2}$ \\ ${ }^{1}$ King Abdulaziz Medical City, King Saud bin Abdulaziz University for Health Science- \\ Nursing College, Jeddah, Kingdom of Saudi Arabia \\ ${ }^{2}$ Queensland University of Technology, School of Nursing, Brisbane, Australia
}

\begin{abstract}
Aims: Cardiovascular disease (CVD) is a major health problem in Saudi Arabia as well as in other highly developed countries. Notably, $42 \%$ of all deaths in Saudi Arabia occur as a result of CVD (WHO, 2011). While there has been a focus on some of the risk factors (smoking and obesity) in Saudi Arabia, there is a paucity of research on secondary prevention practices, healthrelated behaviors and the quality of life for Saudi people following a recent cardiac event. The aim of this study was to examine the health-related behaviors of Saudi people following a recent cardiac event, and identified the factors that influence these behaviors. It was also aim to describe the health-related quality of life of patients in Saudi Arabia after a cardiac event. This paper reports on outcomes of a pilot study undertaken to verify whether the instruments that used to assess health related behaviors and quality of life of Saudi patients following a recent cardiac events is valid and reliable for a Saudi Arabian population after translation into Arabic.
\end{abstract}

Method: A cross-sectional design has been conducted in this study. 60 patients who were visiting the cardiology outpatient clinic at King Fahd General Hospital were recruited.

Results: The pilot study has evaluated selected psychometric properties of the MacNew Arabic version based on criteria recommended by the Scientific Advisory Committee of the Medical Outcomes Trust. This paper will report the results of this testing.

Conclusion: This instrument demonstrated the reliability and validity and used to undertake a large study.

No conflict of interest 
129

\title{
DRUG THERAPY, CLINICAL ASPECTS
}

\section{RISK PROFILE AND VERY LONG-TERM MORTALITY IN YOUNG STEMI PATIENTS UNDERGOING PRIMARY PCI}

 \\ V. Kanjuh ${ }^{3}$, G. Stankovic ${ }^{1}$ \\ ${ }^{1}$ Clinical Center of Serbia, Department of Cardiology, Belgrade, Serbia \\ ${ }^{2}$ Clinical Center of Serbia, Emergency Department- Department of Cardiology, Belgrade, Serbia \\ ${ }^{3}$ Serbia Academy of Sciences and Arts, Board on Cardiovascular Pathology, Belgrade, Serbia
}

\begin{abstract}
Aims: There is paucity of data regarding the risk profile and long-term prognosis of young patients undergoing primary percutaneous coronary intervention (PCI) for ST-elevation myocardial infarction (STEMI).
\end{abstract}

Method: We analyzed 1721 STEMI patients treated with primary $\mathrm{PCI}$ in a high-volume catheterization laboratory in the years 2009-2010. Clinical characteristics and 5-year mortality rate in patients $\leq 40$ years of age were compared to the population of patients aged $>40$ years.

Results: In the studied population there were $5.1 \%$ of patients aged $\leq 40$ years $(n=87)$. Compared to patients $>40$ years of age, young patients tended to be more frequently males $(80.5 \% \mathrm{vs}$ $71.1 \%, \mathrm{p}=0.058)$, were current smokers $(85.1 \%$ vs $51.1 \%, \mathrm{p}<0.001)$ and had lower rates of hypertension, diabetes, renal failure, peripheral vascular disease and Killip $>1$ at presentation. Multivessel coronary disease was less frequent in young patients $(29.6 \%$ vs $62.1 \%, p<0.001)$. Median total ischemic time was similar in patients $\leq 40$ compared to $>40$ years ( 229 vs. 225 minutes, respectively, $p=0.91)$. Mortality rates were significantly lower in patients aged $\leq 40$ years at 5 years $(19.6 \%$ vs. $8.0 \%$, HR $0.39, p=0.01)$, but not at 30 days $(5.4 \%$ vs. $3.4 \%$, HR 0.62 , $\mathrm{p}=0.42$ ). After adjustment for the described baseline risk factors, young age was not an independent predictor of 5 -year mortality (HR $0.51,95 \% \mathrm{Cl} 0.19-1.37, \mathrm{p}=0.18)$.

Conclusion: Patients aged $\leq 40$ years and treated with primary $\mathrm{PCl}$ were predominantly males. Very long-term mortality rates in these patients were lower, and short-term mortality similar, as compared to patients $>40$ years of age.

No conflict of interest 
134

\title{
ANTIPLATELET AND ANTITHROMBOTIC THERAPY \\ TICAGRELOR, BUT NOT CLOPIDOGREL PROTECTS AGAINST REPERFUSION INJURY AND IMPROVES ADVERSE REMODELING AFTER MYOCARDIAL INFARCTION
}

\author{
Y. Birnbaum ${ }^{1}, Y . Y^{2}$ \\ ${ }^{1}$ Baylor College of Medicine, Medicine/ Cardiology, Houston, USA \\ ${ }^{2}$ UTMB, Biochemistry and Molecular Biology, Galveston, USA
}

\begin{abstract}
Aims: Clopidogrel (C) and Ticagrelor (Tic) are P2Y12 ADP receptor antagonists. Tic, but not C, inhibits adenosine cell uptake. Previous data show that $7 \mathrm{~d}$ pretreatment with Tic or one dose administered IP before reperfusion limits infarct size in the rat. We compared the effects of $C$ and Tic, administered just before reperfusion and/or $24 \mathrm{~h}$ after reperfusion and continued for $6 \mathrm{w}$ on cardiac remodeling and fibrosis in a rat model of ischemia/ reperfusion.
\end{abstract}

Method: Rats underwent $30 \mathrm{~min}$ coronary artery ligation. At $25 \mathrm{~min}$ of ischemia rats received intraperitoneal (IP) vehicle without (sham) or with (control) coronary ligation, Tic IP $(30 \mathrm{mg} / \mathrm{kg}$ ) before reperfusion alone (TIP), Tic $(300 \mathrm{mg} / \mathrm{kg} / \mathrm{d})$ oral for $6 \mathrm{w}$, started a day after reperfusion (TPO), Tic IP+PO (TIPPO), or C $(12.5 \mathrm{mg} / \mathrm{kg}$ IP before reperfusion $+62.5 \mathrm{mg} / \mathrm{kg} / \mathrm{d}$ PO for $6 \mathrm{w}$, started a day after reperfusion. LV dimensions and function was assessed by echo at $6 \mathrm{w}$. Collagen III and fibrosis were assessed after $6 \mathrm{w}$.

Results: LVEF was $77.6 \pm 0.9 \% *, 44.8 \pm 3.5 \%, 69.5 \pm 1.6 \%^{*}, 69.2 \pm 1.0 \%{ }^{*}, 76.3 \pm 1.2 \%{ }^{*}$, and $37.4 \pm 3.7 \%$ in the sham, vehicle, TIP, TPO, TIPPO and C treated group, respectively $\left({ }^{*} \mathrm{p}<0.001\right.$ vs. vehicle). Left ventricular diameters showed the same pattern. Percent area fibrosis in the border zone was $1.8+0.2 \%$ in the Sham group, $47.6+2.1 \%$ in the control group and $21.5+0.9^{*}$, $13.1+0.5^{*}, 11.0+0.8^{*}$, and $50.8+2.2 \%$, in the TIP, TPO, TIPPO and C treated group, respectively $\left({ }^{*} \mathrm{p}<0.001 \mathrm{vs}\right.$. vehicle). Similar results were obtain with collagen III mRNA levels.

Conclusion: Oral Tic treatment for $6 \mathrm{w}$ improved heart function and attenuated fibrosis.

Conflict of Interest Disclosure Statement: Grants from Astra Zeneca, BMS, BI 
137

ANTIPLATELET AND ANTITHROMBOTIC THERAPY

THE GARFIELD REGISTRY: A 2015 UPDATE

M. Keltai

Gottsegen Gyorgy Hungarian Institute of Cardiology, Budapest, Hungary

The GARFIELD (Global Anticoagulant Registry In the FIELD) registry is an international, observational, multicenter, prospective study of patients with atrial fibrillation (AF) and one or more additional risk factors for stroke. Enrollment is going on in five independent, sequential, prospective cohorts. The study started in December 2009, with a planned recruitment period of 4 years and a minimum of 2-year follow-up for each patient. Today we are presenting some data of the first four cohorts, with special emphasis on Europe and Hungary.

Up till August 2015 globally 39670 prospective patients were enrolled. The number of patients from Europe and Hungary are shown in the table with the mean age, sex distribution, mean CHA2DC2-VASc and HAS-BLED scores and also the complication rates

\begin{tabular}{|l|l|l|l|l|l|l|l|l|}
\hline & $\begin{array}{l}\text { \# patients } \\
\text { registered }\end{array}$ & Age & Male & $\begin{array}{l}\mathbf{C H A}_{2} \mathbf{D C}_{2-} \\
\text { VASc }\end{array}$ & $\begin{array}{l}\text { HAS- } \\
\text { BLED }\end{array}$ & $\begin{array}{l}\text { Stroke } \\
\text { rate }\end{array}$ & $\begin{array}{l}\text { Major } \\
\text { bleed }\end{array}$ & Mortality \\
\hline Total & 39670 & 69.7 & $55.7 \%$ & 3.2 & 1.4 & 1.37 & 0.86 & 4.0 \\
\hline Europe & 23034 & 70.7 & $54.7 \%$ & 3.3 & 1.4 & 1.36 & 0.99 & 4.27 \\
\hline Hungary & 1005 & 68.6 & $48.0 \%$ & 3.3 & 1.4 & 1.35 & 1.97 & 6.59 \\
\hline
\end{tabular}

Most patients were enrolled by their first AF episode, but permanent (15.0\%), persistent (14.6\%) and paroxysmal $(24.5 \%) \mathrm{AF}$ cases were also registered. The majority of the patients received anticoagulant (AC) or $\mathrm{AC}+$ antiplatelet (AP) treatment. Those with higher $\mathrm{CHA}_{2} \mathrm{DC}_{2}-\mathrm{VASc}$ score were treated by anticoagulants more frequently. $A C$ treatment in the first prospective cohorts was predominantly Vitamin $\mathrm{K}$ antagonist (VKA). The NOAC use was increasing steadily and in the last year over $37 \%$ of the patients received NOAC + with or without AP.

No conflict of interest 


\section{3}

\section{NEW DIAGNOSTIC METHODS}

\section{OUTCOME PREDICTION IN ASYMPTOMATIC DIABETICS WITH SPECIAL REFERENCE TO CORONARY CT ANGIOGRAPHY}

\section{D.A. Halon}

The Ruth and Bruce Rappaport Faculty of Medicine, Technion Institute of Technology, Haifa, Israel

Background and Aims: Asymptomatic type 2 diabetics (DM) are at increased risk for cardiovascular events. Clinical risk scores and coronary artery calcium (CAC) scoring can improve event prediction and coronary CT angiography (CTA) may have incremental value. However, to date routine screening for coronary heart disease (CHD) in diabetics by any modality has not led to improved outcomes.

Method: We followed DM patients for 7 years following CTA to define a cohort at increased risk, who may benefit from intensified preventive/interventional therapy.

Results: Coronary plaque was present in most of 630 patients in the study cohort $(500,79 \%)$. $\mathrm{CHD}$ events (cardiac death, myocardial infarction, unstable angina or new chest pain requiring revascularization) were infrequent occurring overall in $1 \%$ per year. A standard clinical risk score (UK Prospective Diabetic Study, UKPDS) predicted baseline clinical risk. CTA variables were helpful in defining risk over 7 years: CAC score, extent of plaque on CTA and mild plaque calcification were independent outcome predictors and successively improved discrimination on $\mathrm{ROC}$ analysis. A multivariate model including UKPDS, total plaque extent and plaque characterization had good prediction, discrimination and reclassification for MACE (Overall net reclassification improvement $27.3 \%$ (3 risk categories) vs UKPDS + CAC score alone). Event rate in the upper decile of patient risk was $28 \%$ vs $6.5 \%$ in the total cohort.

Conclusion: Addition of CTA to risk assessment defined by UKPDS and CAC score had incremental value in identification of a DM cohort at high risk for CHD events who may benefit from further study or intervention.

No conflict of interest 


\title{
144
}

\section{NEW DIAGNOSTIC METHODS}

\section{CLINICAL VALUE OF CAC MEASUREMENT: WHAT HAVE WE LEARNT?}

\author{
J. Shemesh
}

The Grace Ballas Cardiac Research Unit, Sheba Medical Center, Israel

Coronary artery calcification (CAC) is the best surrogate marker of the total burden of coronary atherosclerosis. Its presence in asymptomatic subjects indicates the existence of subclinical disease and its quantity reflects the extent and the chronicity of the disease. After 3 decades of intensive research in this field several important understandings are widely accepted and some of them are clinically applicable. Primary prevention: The most important clinical value of CAC measuring is the diagnosis of early subclinical CAD. This information further improve the selection of those who are at higher risk for future cardiovascular $(\mathrm{CV})$ events and who are the best candidates for intensive preventive therapy. Indeed prospective population studies as the MESA, Recall, Amsterdam studies and many others demonstrated the clear higher predictive value of CAC score over the classical and novel risk factors. Equally important is the absence of CAC which indicates an excellent prognosis in asymptomatic as well in high risk patients such as diabetics, hypertensive, smokers, elderly and even CKD patients. Atherosclerosis and statin treatment: The presence and extensiveness of CAC reflect the entire spectrum of genetic and life time exposure to the numerous acquired risk factors of which atherosclerosis is the final result . While the presence of CAC can refine the selection of the best beneficial group from intensive statin therapy, the absence of CAC reclassifies those who cannot tolerate statin into a lower risk category giving the opportunity to reconsider the statin use and intensity. Clinical significance of CAC progression: The new modalities of the intra vascular ultra sound techniques enable sophisticated tracking of the CAC progression. This, shades a new light into the obscure role of calcium in the coronary atherosclerotic plaques. Recent studies which compare intensive statin treatment to usual care in post PCI patients and in those with stable CAD, demonstrated that intensive statin treatment increased CAC progression which was related to reduction in $\mathrm{CV}$ hard events. This, strengthen previous observations that extensive calcifications clinically manifested as chronic $\mathrm{CAD}$ ( $\mathrm{AP}, \mathrm{PCl}, \mathrm{CABG}$ ) while acute coronary syndrome are mostly occurs on the ground of mild or moderate calcifications. It can be hypothesized that CAC has a role in the healing process stabilizing the inflamed plaques and thus reduces the incidence of ACS. CAC should be reported in all chest CT: CAC can be detected and quantified by all CT devices whenever low dose chest CT is performed. It is particularly valuable when combined with early lung cancer detection. The future: The current CT modalities cannot detect the vulnerable plaques. This, remains the major challenge for the prevention of sudden death and acute MI. 
145

NEW DIAGNOSTIC METHODS

MYOCARDIAL FIBROSIS IMAGING WITH MRI: CONTRAST OR NON-CONTRAST?

J. Zheng

Mallinckrodt Institute of Radiology, Saint Louis, MO, USA

Aims: Cardiac fibrosis is one of the hallmarks of pathological left ventricular remodeling, which plays a significant role in the myocardial response to injury, such as coronary artery disease. Excessive cardiac fibrosis leads to progression of heart failure. Therefore, non-invasive imaging methods to quantitatively and reproducibly assess at an early stage the presence, extent, and turnover of myocardial fibrosis are needed for the stratification of risk of heart failure and also to monitor the effects of treatment in patients.

Method: For the last decades, MRI T1 mapping technique was developed for the quantification of extracellular volume fraction (ECV), a surrogate marker for myocardial fibrosis. This technique requires administration of gadolinium contrast agent to calculate $E C V$, which may be contraindicated in patients with impaired renal function (e.g., in heart failure, diabetes, and endstage renal dysfunction). It is also prone to errors caused by dosage of contrast agent, measurement timing, and renal clearance rate. Non-contrast methods to detect myocardial fibrosis are still on early stage and a few reports have identified native T1 and spin-locking T1r as imaging contrast for fibrosis. In this presentation, contrast-agent-based and non-contrast-agent MRI methods for imaging myocardial fibrosis will be briefly reviewed. A new non-contrast fibrosis index will be introduced for the sensitive detection of fibrosis, particularly the diffuse fibrosis.

Results: Studies from us and others will be included together to demonstrate the promise of these methods for the diagnosis of myocardial fibrosis.

Conclusion: MRI fibrosis imaging are reliable and quantitative methods for assessment of myocardial fibrosis.

No conflict of interest 
146

NEW DIAGNOSTIC METHODS

\title{
DUAL UTILIZATION OF EXERCISE ECHOCARDIOGRAM AND MYOCARDIAL PERFUSION IN DIRECTING MANAGEMENT OF ISCHEMIA IN A CARDIOLOGY PRACTICE
}

\author{
Y. Charuzii, R. Gao, J. Mirocha \\ Cedars-Sinai Heart Institute, Los Angeles, CA, USA \\ In patients with suspected ischemia, the data derived from one type of imaging test may be \\ insufficient to guide management. In 100 patients, two imaging techniques were utilized to assess \\ the extent of ischemia and help determine proper clinical management. 207 dual studies \\ consisting of both a myocardial perfusion test and an exercise echocardiogram were performed \\ on the same 100 patients. The median patient age at testing was 69 , and eighty-two percent were \\ male. The tests were conducted within two months of each other. Myocardial perfusion was \\ evaluated by exercise SPECT, adenosine SPECT, or a PET scan. Exercise echocardiograms \\ were performed by using a treadmill and BRUCE protocol and imaging before and immediately \\ after exercise. Ischemia by perfusion not associated with exercise wall motion abnormalities was \\ considered mild. If ischemia was seen with both techniques, it was considered significant and \\ handled appropriately. Fifty-four percent of the nuclear tests and thirty-two percent of exercise \\ echocardiograms were abnormal. In approximately twenty-five percent of the dual studies, a \\ positive nuclear test was not associated with wall motion abnormalities in the exercise \\ echocardiogram, thus leaving the diagnosis of ischemia open.
}

No conflict of interest 


\title{
147 \\ NEW DIAGNOSTIC METHODS \\ THE ROLE OF IVABRADINE IN MYOCARDIAL PERFUSION
}

\author{
J. Camici \\ Università Vita Salute San Raffaele, Milan, Italy \\ Heart rate $(\mathrm{HR})$ is a main determinant of myocardial oxygen consumption and has a fundamental \\ role in the development of demand ischaemia in patients with coronary artery disease (CAD). \\ Increased HR is associated with cardiovascular morbidity and mortality and with increased risk for \\ heart failure (HF). Studies in dogs treated with ivabradine, a specific inhibitor of the If-current that \\ reduces HR, have demonstrated a reduction of left ventricular (LV) dyskinesia and duration of \\ post ischaemic myocardial stunning following treadmill exercise. More recently, we have \\ demonstrated that ivabradine limits exercise-induced LV dysfunction and post-ischaemic stunning \\ in patients with CAD. Previous studies in a canine model have shown that the improvement in \\ stunning following intravenous administration of ivabradine was accompanied by amelioration of \\ subendocardial blood flow.
}

The severity and duration of post-ischaemic stunning are known to depend on the severity and duration of the preceding ischaemic episode. Therefore, it can be hypothesized that in our patients the reduction of HR achieved after ivabradine resulted in a more favorable balance between oxygen demand and supply which, in turn, attenuated the degree of myocardial ischaemia and stunning. CAD is the major cause of chronic LV systolic dysfunction and HF due to either irreversible myocyte loss with scar formation and/or hibernating myocardium, i.e. chronically dysfunctional, but viable tissue which improves function after revascularization. Studies using positron emission tomography have demonstrated that the coronary flow reserve to hibernating myocardium is severely reduced and it has been hypothesized that repeated episodes of demand ischaemia followed by chronic stunning could be a potential mechanism of hibernation. Accordingly, we have previously demonstrated that, in patients with CAD and exercise-inducible ischaemia, two consecutive episodes of ischaemia lead to more severe and prolonged stunning. Therefore, it might be surmised that ivabradine could have a positive effect on chronic post-ischaemic LV dysfunction through a reduction of demand ischaemia and stunning, ultimately reducing hibernation. These mechanisms could explain, at least in part, the positive results of the SHIFT trial in which almost $70 \%$ of the patients had post-ischaemic HF.

No conflict of interest 


\title{
148
}

\section{NEW DIAGNOSTIC METHODS}

\section{SPECKLE TRACKING ECHOCARDIOGRAPHY - 10 YEARS PERSPECTIVE. WHAT HAVE WE} LEARNED AND WHERE ARE WE GOING?

\author{
Z. Vered \\ Department of Cardiology, Assaf Harofeh Medical Center, Zerifin, Israel
}

Since the original publications in 2004, speckle tracking echocardiography has developed into a real-time quantitative automatic software capable of measuring strain and strain rate, and also more advanced measurements such as systolic and diastolic LV twist, rotation and torsion, as a measure of myocardial contraction and relaxation. We and others have shown that the technique is capable of differentiating between normal subjects and patients post myocardial infarction and correlates well with left ventricular ejection fraction. More recent research has focused on the accurate diagnosis of acute ischemia/reperfusion and also on myocardial viability. In studies with rats subjected to acute Ml followed by reperfusion analysis of the LV in 3 layers of strain was shown to be advantageous in differentiating sub-endocardial as opposed to transmural global strain in these situations. In another more recent work, 10 pigs were subjected to balloon occlusion of the LAD for 90 minutes followed by reperfusion. Serial echocardiographic studies were performed before occlusion, immediately after and then at 24 hours, 30 and 60 days post reperfusion. Detailed histological analysis of the LV was performed. Time-to peak circumferential strain analysis after reperfusion could differentiate between stunned (areas of hypo-contraction improving later) and infracted myocardium (areas with significant scar formation on pathology). Clinical studies have focused on its application during exercise and dobutamine stress studies to enhance the diagnosis of ischemia and the assessment of LV function in asymptomatic severe aortic stenosis to assist determining the optimal time to surgery. 3D/4D analysis of strain/strain rate and the implementation of these techniques into standard conventional and stress echocardiography will no doubt further enhance its clinical applicability and diagnostic capability.

No conflict of interest 
152

MITOCHONDRIAL DYNAMICS AND HEART DISEASE

\title{
MITOCHONDRIAL CA2+ HOMEOSTASIS AND ROS FORMATION BETWEEN HEART INJURY AND PROTECTION
}

\author{
F. Di Lisa \\ University of Padova, Padova, Italy \\ Mitochondrial $\mathrm{Ca} 2+$ overload is considered a relevant factor in determining the loss of \\ cardiomyocyte viability occurring during post-ischemic reperfusion. This concept is based mostly \\ on the protection afforded by Ruthenium compounds that inhibit mitochondrial $\mathrm{Ca} 2+$ uptake. \\ However, Ruthenium compounds affect also other processes involved in regulating intracellular \\ $\mathrm{Ca} 2+$ homeostasis. On the other hand, a slight increase in intracellular $\mathrm{Ca} 2+$ has been shown to \\ mimic the cardioprotective effects elicited by ischemic preconditioning (IPC). Similarly, also a mild \\ increase in reactive oxygen species (ROS) seems to be required for IPC protection, while a \\ severe oxidative stress is undoubtedly associated with cardiomyocyte injury. We have \\ investigated the relationship between mitochondrial $\mathrm{Ca} 2+$ uptake and cardiac ischemic injury by \\ overexpressing the mitochondrial $\mathrm{Ca} 2+$ uniporter $(\mathrm{MCU})$ in neonatal cardiac myocytes and intact \\ hearts subjected to protocols of ischemia/reperfusion (I/R). MCU overexpression reduced I/R- \\ induced cell death both in NRVMs and intact hearts. The decreased susceptibility to reperfusion \\ injury appears to represent a preconditioning-like effect that might result from a slight increase in \\ mitochondrial ROS levels. The increased ROS levels in response to the augmented $\mathrm{Ca} 2+$ uptake \\ is linked to an enhanced activity of survival kinases.
}

No conflict of interest 


\title{
153
}

\section{MITOCHONDRIAL DYNAMICS AND HEART DISEASE}

\section{CARDIOPROTECTION: A TALE OF 2 MECHANISMS}

\author{
J. Hill \\ UT Southwestern Medical Center, Dallas, TX, USA
}

Heart failure is increasing in incidence and prevalence rapidly around the globe. A devastating syndrome that emanates from numerous forms of heart disease, heart failure carries a mortality of approximately $50 \%$ at 5 years. Coronary artery disease is the leading cause of heart failure with reduced ejection fraction (HFrEF). Despite the use of novel therapies and devices, there appears to be a ceiling on our ability to mitigate infarct size by restoration of coronary artery patency alone. Reperfusion injury is a major contributor to infarct size, approaching $50 \%$ of total injury burden. However, as yet no standard therapy exists.

We will discuss two recent findings from our lab focusing on cardioprotection in ischemia/reperfusion (I/R) injury. We have discovered a novel link between the Unfolded Protein Response (UPR) and the Hexosamine Biosynthetic Pathway (HBP) governing protein O-

GlcNAcylation, an established cytoprotective mechanism. We demonstrate that the UPR triggers activation of the HBP by means of Xbp1s-dependent transcription of genes coding for key, ratelimiting enzymes. We further demonstrate a physiologic role for the UPR-HBP axis, by showing that acute stimulation of Xbp1s in the heart confers robust cardioprotection in part through induction of the HBP.

Second, we will discuss the role of reversible protein acetylation in I/R injury. We will discuss data demonstrating that an FDA-approved histone deacetylase inhibitor reduces myocardial infarct size in a large animal model of I/R injury. Re-activation of I/R-triggered down-regulated autophagy during reperfusion is a major underlying mechanism.

No conflict of interest 
160

LIPIDS, LIPOPROTEINS AND ATHEROSCLEROSIS

\title{
ANTI-ATHEROSCLEROTIC AND ANTI-INFLAMMATORY EFFECTS OF A NOVEL BROMODOMAIN EXTRA-TERMINAL (BET) PROTEIN INHIBITOR RVX-208, IN HYPERLIPIDEMIC APOE DEFICIENT MICE AND HUMANS
}

\author{
R. Jahagirdar ${ }^{1}$, S. Azhar ${ }^{2}$, S. Attwell ${ }^{3}$, E. Kulikowski ${ }^{3}$, M. Sweeney ${ }^{4}$, J. Johansson ${ }^{4}$, \\ N.C.W. Wong ${ }^{4}$ \\ ${ }^{1}$ Resverlogix Corp, Pharmacology, Calgary, Canada \\ ${ }^{2}$ VA Palo Alto Health Care System, Medicine, Palo Alto, USA \\ ${ }^{3}$ Resverlogix Corp, Biology, Calgary, Canada \\ ${ }^{4}$ Resverlogix Corp, Clinical, Calgary, Canada
}

\begin{abstract}
Aims: RVX-208, a selective BD2 inhibitor, competes for binding of acetylated lysine to BET proteins. In vervet monkeys and humans, RVX-208 raises ApoA-I, pre $\beta-H D L$ and HDL-c. Posthoc analysis of human trials data showed RVX-208 markedly reduces major adverse CVD events (MACE) by 55\% in CVD patients but more so in those with diabetes mellitus. Thus biologic plausibility for benefits of RVX-208 on MACE was examined in hyperlipidemic ApoE-/- mice to assess aortic lesions, anti-inflammatory effects in a carotid ligation model of inflammation and U937 and Human Aortic Endothelial Cells (HAEC).
\end{abstract}

Method: ApoE-/- mice were administered RVX-208 at $150 \mathrm{mg} / \mathrm{kg}$ b.i.d. orally for 12 weeks, for 14 weeks in atherosclerosis established mice and for 2 weeks in carotid artery ligation inflammation model. HAECs were stimulated with TNFa and U937 with LPS to assess effects on cytokines by RVX-208.

Results: RVX-208 significantly reduced aortic lesions along with a 2-fold rise in HDL-c levels, lower LDL-C by $1 / 2$ and reduced cytokines haptoglobin, VCAM-1, IL-18, MIP1 $\alpha$ and SAP. RVX208 administered for 14 weeks reduced aortic lesions by $39 \%$ and lowered the cytokines IP-10, MIP1 $\beta$ and MDC. In the carotid ligation model of inflammation, 2 weeks of RVX-208 administration reduced expression of aortic IL-6 and VCAM-1. RVX-208 suppressed expression of VCAM-1 and MCP-1 in HAECs with an IC50 of 1 and 10 uM respectively, while in U937 cells the IC50 for IL-6 was $1 \mathrm{uM}$.

Conclusion: In summary, effect of RVX-208 in in-vitro and in-vivo models show coordinated mechanisms of anti-atherogenic and anti-inflammatory activity of a selective BET inhibitor.

Conflict of Interest Disclosure Statement: As part of employee contract I own shares in Resverlogix Organization 
161

\title{
LIPIDS, LIPOPROTEINS AND ATHEROSCLEROSIS
}

\section{NIACIN ADMINISTRATION SIGNIFICANTLY REDUCES OXIDATIVESTRESS IN PATIENTS WITH HYPERCHOLESTEROLEMIA AND LOWLEVELS OF HIGH-DENSITY LIPOPROTEIN CHOLESTEROL}

\author{
S. Hamoud ${ }^{1}$, M. Kaplan², E. Meilin' ${ }^{3}$, A. Hassan', R. Torgovicky4, R. Cohen ${ }^{5}$, T. Hayek ${ }^{1}$ \\ ${ }^{1}$ Rambam health care campus, Internal medicine E, Haifa, Israel \\ ${ }^{2}$ Rambam health care campus, clinical biochemistry laboratory, Haifa, Israel \\ ${ }^{3}$ Technion- faculty of medicine, Lipid research laboratory, Haifa, Israel \\ ${ }^{4}$ Merk- Sharp and Dohme, medical department, Petah Tiqva, Israel \\ ${ }^{5}$ Merck- Sharp and Dohme, medical department, Hod Hasharon, Israel
}

Aims: To evaluate the effect of niacin treatment in patients with low levels of HDL cholesterol (HDL-C; $<40 \mathrm{mg} \%$ ) on their lipid profile and oxidative stress status

Method: Seventeen patients with hypercholesterolemia and low HDL-C and 8 healthy control subjects were enrolled in the study. The patients were treated with niacin for 12 weeks. Lipid profile, oxidative stress and C-reactive protein (CRP) levels were determined at the time of enrollment, and 2 and 12 weeks after initiation of niacin treatment

Results: Niacin treatment in hypercholesterolemic patients caused a significant increase inHDL$\mathrm{C}$ and apolipoprotein A1 levels, and a decrease in triglyceridelevels. Niacin also significantly reduced oxidative stress, as measured by a significant decrease in the serum content of thiobarbituric acidreactive substances, lipid peroxides and paraoxonase activity, comparedwith the levels before treatment

Conclusion: Niacin treatment in ypercholesterolemic patients with low HDL levels caused a significant decrease in their oxidative stress status.

No conflict of interest 
162

\title{
LIPIDS, LIPOPROTEINS AND ATHEROSCLEROSIS
}

\section{REDUCED EXPRESSION OF PEROXIREOXIN 2 IN AORTIC SMOOTH MUSCLE CELLS AT THE ONSET OF ATHEROGENESIS IN APOE-KNOCKOUT MICE}

\author{
H. Itabe, R. Kato, M. Hayashi, T. Obama, T. Aiuchi, T. Yamaguchi \\ Showa University School of Pharmacy, Biological Chemistry, Tokyo, Japan
}

\begin{abstract}
Aims: It is believed it takes many years to build up atherosclerotic plaques. Our previous study showed that plasma level of oxidized LDL increases transiently in apoE-knockout mice fed normal chow diet just before atheorslcerotic lesions begin to develop around 20 weeks of age. This study aims to search very early changes in aortic tissues involved in atherogenesis.
\end{abstract}

Method: Protein profiles of root and abdominal regions of aortas from 10-week old apoEknockout mice were analyzed. After 2-dimensional electrophoresis several spots showing different abundance were identified using MALDI-TOF-MS. Changes in the protein expression levels were immunohistochemically examined.

Results: In aortas, atherosclerosis develops earlier in root and arch regions than in thoracic and abdominal regions. One of the proteins which reduced their expression levels in the root regions was identified as peroxiredoxin 2 (Prx2). Prx2 is expressed in medial smooth muscle cells (SMC). Since Prx2 is a major cellular antioxidant enzyme which scavenges hydrogen peroxide, the reduced Prx2 level suggests increased oxidative stress in the certain spots of aorta. Prx2 expressed strongly in the root region at 4 weeks of age, and its expression level reduced at week 10 , whereas oxidation products in the regions increased by week 10. In addition to Prx2, expression levels of alpha-SM actin and transgelin-2, two well-known differentiation markers for $\mathrm{SMC}$, were lower in the root than the abdominal regions.

Conclusion: These observations suggest that oxidative stress caused by decrease in Prx2 may dinduce dedifferentiation of aortic SMC at the very early phase of atherogenesis in apoE-knockou mice.

No conflict of interest 
163

\title{
LIPIDS, LIPOPROTEINS AND ATHEROSCLEROSIS \\ CHARACTERIZATION OF A FILTRATION-BASED POINT-OF-CARE DEVICE FOR THERAPEUTIC ANGIOGENESIS
}

\author{
G. Spaltro ${ }^{1}$, S. Straino ${ }^{2}$, E. Gambini', B. Bassetti ${ }^{1}$, C. Quarti ${ }^{3}$, G. Pompilio ${ }^{1}$ \\ ${ }^{1}$ Centro Cardiologico Monzino - IRCCS, Vascular Biology and Regenerative Medicine Unit, \\ Milano, Italy \\ ${ }^{2}$ Istituto Dermopatico dell'Immacolata, Vascular Pathology Laboratory, Rome, Italy \\ ${ }^{3}$ Pall Corporation, Pall Italia, Buccinasco, Italy
}

\begin{abstract}
Aims: The Pall Celeris ${ }^{\mathrm{TM}}$ system is a filtration-based point-of-care device designed to obtain a high concentrate of peripheral blood total nucleated cells (PB-TNCs). We have characterized the Pall Celeris ${ }^{\mathrm{TM}}$-derived TNCs for their in vitro and in vivo angiogenic potency.

Method: PB-TNCs isolated from healthy donors were characterized through the use of flow cytometry and functional assays, aiming to assess migratory capacity, ability to form capillary-like structures, endothelial trans-differentiation and paracrine factor secretion. In a hind limb ischemia mouse model, we evaluated perfusion immediately and 7 days after surgery, along with capillary, arteriole and regenerative fiber density and local bio-distribution.
\end{abstract}

Results: Human PB-TNCs isolated by use of the Pall Celeris ${ }^{\mathrm{TM}}$ filtration system were shown to secrete a panel of angiogenic factors and migrate in response to vascular endothelial growth factor and stromal-derived factor-1 stimuli. Moreover, after injection in a mouse model of hind limb ischemia, PB-TNCs induced neovascularization by increasing capillary, arteriole and regenerative fiber numbers, with human cells detected in murine tissue up to 7 days after ischemia.

Conclusion: The Pall Celeris ${ }^{\mathrm{TM}}$ system represent a novel, effective and reliable point-of-care device to obtain a PB-derived cell product with adequate potency for therapeutic angiogenesis.

No conflict of interest 
164

\title{
LIPIDS, LIPOPROTEINS AND ATHEROSCLEROSIS \\ MOLECULAR BASIS OF DIET, EXERCISE AND LIFESTYLE INTERVENTIONS IN ATHEROSCLEROTIC CARDIOVASCULAR DISEASE
}

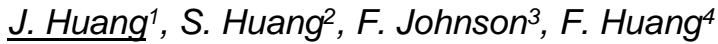 \\ ${ }^{1}$ Saint Louis University School of Medicine, Biochemistry and Molecular Biology, St. Louis, USA \\ ${ }^{2}$ Auxagen- Inc., Research, St. Louis, USA \\ ${ }^{3}$ Saint Louis University School of Medicine, Surgery, St. Louis, USA \\ ${ }^{4}$ Broad Institute of Harvard and MIT, Department of Medical Oncology- Dana- \\ Farber Cancer Institute- Harvard Medical School, Boston, USA
}

\begin{abstract}
Aims: Circulating TGF- $\beta$ is a cytokine that inhibits atherosclerosis. Hypercholesterolemia and high levels of dietary trans fats cause atherosclerosis, at least in part, by suppressing TGF- $\beta$ activity in arterial endothelium. Dynasore, a TGF- $\beta$ enhancer, retards atherosclerosis without altering high plasma cholesterol levels in ApoE-null mice. Interventions (prudent diet, exercise and lifestyle) decrease the risk of atherosclerosis but the molecular basis is unclear. We tested the hypothesis that TGF- $\beta$ activity enhancers inhibit atherosclerosis.
\end{abstract}

Method: Many agents (including betulinic acid, DMSO, aspirin, resveratrol, apocynin, vitamin D3 and polyunsaturated fatty acids), moderate drinking of red wine, low-dose aspirin and high plasma HDL levels are known to inhibit atherosclerosis in humans and animals. We determined the TGF- $\beta$ enhancer activity of these agents and factors by measuring their effects on TGF- $\beta$ stimulated expression of luciferase reporter genes, P-Smad2 and PAI-I mRNA, and examining their ability to recruit TGF- $\beta$ receptors from lipid rafts/caveolae to non-lipid raft microdomains using cell-surfacel ${ }^{125}$-TGF- $\beta$ affinity labeling, sucrose density gradient ultracentrifugation and indirect immunofluorescence staining in Mv1Lu cells.

Results: Betulinic acid, DMSO, ethanol, resveratrol, apocynin, aspirin, vitamin D3, and $y$-linolenic acid enhance TGF- $\beta$ activity (2-8 fold) at their optimal concentrations. These agents and HDL enhance TGF- $\beta$ activity by recruiting TGF- $\beta$ receptors from intracellular vesicles and/or lipid rafts/caveolae to non-lipid raft microdomains where canonical signaling occurs.

Conclusion: Diets containing natural TGF- $\beta$ enhancers (fruits/vegetables/nuts rich in triterpenoids/polyphenols/antioxidants/polyunsaturated fatty acids), synthetic TGF- $\beta$ enhancers (such as aspirin/resveratrol/cyanidin/vitamin D3), moderate drinking of red wine, and exercise (which increases plasma HDL levels) are now known to prevent and treat atherosclerotic cardiovascular disease.

Conflict of Interest Disclosure Statement:

Shuan Shian Huang and Jun San Huang had an equity position in Auxagen Inc. during the time the research was carried out. 
165

LIPIDS, LIPOPROTEINS AND ATHEROSCLEROSIS

\title{
URBAN-RURAL DIFFERENCE IN LIPID LEVELS AND PREVALENCE OF DYSLIPIDEMIA IN SOUTHERN THAI POPULATION
}

\author{
N. Jeenduang \\ School of Allied Health Science and Public Health - Walailak University, Medical Technology, \\ Thasala, Thailand
}

Aims: Dyslipidemia is one of the major risk factors for cardiovascular disease (CVD). This study was aim to investigate the lipid levels and the prevalence of dyslipidemia in urban and rural subjects in Southern Thailand.

Method: A cross-sectional study was conducted in 629 subjects (327 urban; 302 rural), ranging between 19 and 84 years of age. Blood pressure, anthropometric indices, fasting blood sugar (FBS), and lipid levels were measured. Dyslipidemia was diagnosed using NCEP ATPIII guidelines.

Results: Urban subjects had significantly higher total cholesterol (TC), low density lipoprotein cholesterol (LDL-C), and TC/HDL-C ratio but lower waist circumference (WC), body mass index (BMI), diastolic blood pressure (DBP), triglyceride (TG), high density lipoprotein cholesterol (HDL$C)$, and FBS compared to rural subjects $(p<0.05)$. The most common dyslipidemia in both urban and rural subjects were high TC and high LDL-C. Urban subjects had significantly higher prevalence of high TC, high LDL-C, low HDL-C, and high TC/HDL-C ratio but lower the prevalence of high TG compared to rural subjects $(p<0.05)$. Multiple logistic regression analysis showed that menopause, and urban residents were associated with increased odds of high TC and high LDL-C. Obesity, and hypertension had greater odds of having high TG. In addition, women, overweight, obesity, and urban residents were found associated with increased odds of low HDL-C.

Conclusion: The present study demonstrated a significant difference in lipid levels and the prevalence of dyslipidemia between urban and rural subjects. This variation may be due to difference in lifestyle and sociodemographic factors among this population.

No conflict of interest 
166

LIPIDS, LIPOPROTEINS AND ATHEROSCLEROSIS

\section{TOLL-LIKE RECEPTOR-4 IS UPREGULATED IN PLAQUE DEBRIS OF PATIENTS WITH ACUTE CORONARY SYDROME MORE THAN TOLL-LIKE RECEPTOR-2}

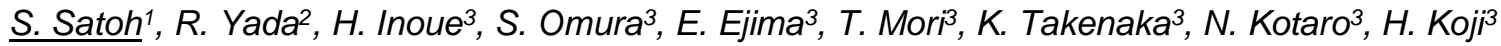

${ }^{1} \mathrm{NHO}$ Kyushu Medical Center, Cardiology and Clinical Research Insitute, Fukuoka, Japan

${ }^{2} \mathrm{NHO}$ Kyushu Medical Center, Clinical Research Insitute, Fukuoka, Japan

${ }^{3} \mathrm{NHO}$ Kyushu Medical Center, Clinical Research Institute, Fukuoka, Japan

Aims: Atherosclerosis is a disease characterized by inflammation in the arterial wall. Atherogenesis is dependent on the innate immune response involving activation of Toll-like receptors (TLRs) and the expression of inflammatory proteins, those may lead to acute coronary syndrome (ACS). We investigated the expression level of TLR-4 in ACS, as compared with TLR-2 and patients with stable angina.

Method: Consecutive 58 patients who underwent primary percutaneous coronary intervention $(\mathrm{PCl}, \mathrm{n}=29)$ because of $\mathrm{ACS}$ and elective $\mathrm{PCI}(\mathrm{n}=29)$ because of stable angina using a filterdevice distal protection device system were prospectively analyzed. mRNA levels of TLR-2 and TLR-4 in debris containing various inflammatory tissues entrapped in the filter device were altogether analyzed using real-time PCR.

Results: There were no significant differences in age, sex distribution, between stable angina and ACS groups. TLR-4 expression levels were higher in patients with ACS than in patients with stable angina.

Conclusion: TLR-4 might play a more important role than TLR-2 in atherogenesis, especially in ACS.

No conflict of interest 
167

\title{
LIPIDS, LIPOPROTEINS AND ATHEROSCLEROSIS
}

\section{LIPID PROFILE AND DIETARY PATTERNS\&ACUTE; IMPROVEMENT AFTER A CARDIAC REHABILITATION PROGRAM IN CARDIOVASCULAR HIGH-RISK PATIENTS}

\author{
E. Alegria-Barrero',2, M.A. Blazquez', J. Ruiz-Garcia ${ }^{1,2}$, R. Teijeiro-Mestre ${ }^{1}$, L. Borrego', \\ M. Vega ${ }^{1}$, C. Sánchez-Rodríguez ${ }^{1}$, C. González-Cristofol', M.A. San Martin ${ }^{4}$ \\ ${ }^{1}$ Hospital Universitario de Torrejon, Cardiology, Madrid, Spain \\ 2Universidad Francisco de Vitoria, Medicine, Madrid, Spain \\ ${ }^{3}$ Hospital Universitario de Torrejon, Physical Medicine and Rehabilitation, Madrid, Spain \\ ${ }^{4}$ Hospital Universitario de Torrejon, Madrid, Spain
}

Aims: To investigate how a cardiac rehabilitation program can improve lipid profile and dietary patterns in cardiovascular high-risk patients.

Method: From 2013 to 2015, 153 patients were included in a hospital-based cardiac rehabilitation program (CRP). Median duration of the program was 8 weeks. Increasing phyisical exercise was programmed according to a baseline exercise test, based on maximal heart rate achieved. All patients trained with continuous EKG monitoring. Lipid profile was evaluated before and after the program. Dietary patterns were investigated using an original questionnaire on Mediterranean diet (maximum score: 100 points). Specialized cardiac rehabilitation nurses and a nutritionist closely followed-up daily diet through patients' diaries. Weekly seminars were also scheduled.

Results: $75.2 \%$ of the patients were male with a median age of 57 years (IQR 14). $87 \%$ of the patients had a previous history of ischemic heart disease. Mean ejection fraction was 0.57 . Lipid profile improved significantly after the program (see Table). Additionally, patients improved adherence to the recommendations on cardiac healthy diet, obtaining higher scores on the original test evaluating (60.1 vs $80.9, p<0.001)$. Moreover, diet and physical exercise over 8 weeks achieved significant cholesterol, triglycerides and LDL-cholesterol reductions; and HDLcholesterol increase (see Table).

\begin{tabular}{|c|c|c|c|}
\hline & PRE $^{*}$ & POST $^{*}$ & P \\
\hline WEIGHT & $83.7(16.4)$ & $81.9(15.6)$ & $<0.001$ \\
\hline BMI (kg/m ${ }^{2}$ ) & $29.6(4.7)$ & $28.8(4.5)$ & $<0.001$ \\
\hline WAIST CIRCUMFERENCE (cm) & $102.8(11.5)$ & $97.5(10.9)$ & $<0.001$ \\
\hline GLUCOSE & $105.4(25.4)$ & $102.4(22.2)$ & 0.13 \\
\hline HBA1 (\%) & $6(0.9)$ & $5.8(0.8)$ & 0.013 \\
\hline CHOLESTEROL (mg/dl) & $145.7(33.1)$ & $137.4(26.2)$ & 0.001 \\
\hline LDL-CHOLESTEROL (mg/dl) & $79.7(25.2)$ & $71.7(19.7)$ & $<0.001$ \\
\hline HDL-CHOLESTEROL (mg/dl) & $39.6(12.2)$ & $44.2(12.2)$ & $<0.001$ \\
\hline TRIGLYCERIDES (mg/dl) & $130.2(87.2)$ & $106.2(66.8)$ & 0.001 \\
\hline
\end{tabular}

Values are expressed as mean (standard deviation)

Finally, a significant reduction of body mass index (29.6 vs $28.8, p<0.001)$ and waist circumference (102.8 vs $97.5, \mathrm{p}<0.001)$ was achieved.

Conclusion: Cardiac rehabilitation significantly improved lipid profile, improved dietary patterns and anthropometric measurements (weight, BMI and waist circumference) in high-risk patients. 
No conflict of interest 
168

\title{
LIPIDS, LIPOPROTEINS AND ATHEROSCLEROSIS
}

\section{POLICOSANOL A NEW INHIBITOR CANDIDATE FOR VASCULAR CALCIFICATION IN EXPERIMENTAL MODEL OF DIABETIC HYPERLIPIDAEMIC RATS}

\author{
M.M. Elseweidy, N. Zein, S.I. Aldohmy, M.M. Elsawy \\ Biochemistry and Pharmacognosy Departments Faculty of Pharmacy and Biochemistry \\ Department, Faculty of Science, Zagazig University, Zagazig, Egypt
}

\begin{abstract}
Vascular calcification (VC) is relevant pathophysiological process associated with coronary atherosclerosis and leading to cardiovascular morbidity and mortality. Previously it was considered a passive degenerative condition of aging but now it is considered a complex, actively controlled intracellular molecular process, involving the differentiation of macrophages and vascular smooth muscle cells (VSMCS) into osteoclast-like cells, similar to that occurs in bone formation. The underlying pathophysiological mechanisms leading to VC can be broadly described as: (1) Elevation in serum $\mathrm{Ca}++$ and P levels. (2) Induction of osteogenesis. (3) Inadequate inhibition of mineralization process. (4) The migration and differentiation of macrophages and VSMCS into osteoclast- like cells.
\end{abstract}

Accepted evidence has indicated that cholesterol in vascular tissue stimulates calcification and plays certain role in calcium crystals deposition. In vitro study indicated that 25 -hydroxy cholesterol was found in coronary atherosclerotic lesions and accelerating valvular calcification . Vascular calcification is increased also in diabetic patients leading to increased morbidity and mortality rates as compared to diabetic patients without calcification.

Policosanols are a mixture of aliphatic alcohols derived from purified sugar cane, have been shown to decrease the risk of atheroma formation by reducing platelet aggregation, endothelial damage, and foam cell formation in experimental animals in addition to its inhibition of cholesterol biosynthesis and enhancement of receptor-dependent LDL processing.

Its efficacy, safety and tolerability have been established in controlled clinical studies conducted on healthy volunteers, hypercholesterolaemic patients or with dyslipidaemia secondary to type II diabetes mellitus.

The present study was undertaken to investigate the effect of policosanol, on hyperlipidemia, oxidative stress, inflammatory degree and aortic calcification of diabetic hyperlipidaemic rat models as compared to atorvastatin (synthetic hypolipidaemic drug).

For drugs assessment Certain Biomarkers were selected, namely lipogram profile ,CRP , TNFa, MDA , GSH , ALP , PTH , Vitamin D, histopathological examination and histochemical demonstration of calcium deposition in aortic tissues. Both drugs induced significant improvement of lipogram profile, decreased TNF- $\alpha, C R P, A L P, P T H$, MDA along with significant increase of Vitamin D and GSH.

Atorvastatin treatment resulted in the reduction of density, number of foam cells, inflammation and calcium deposition in the aorta. However policosanol group showed an improvement of intimal lesion and in agreement with previous study and nearly no calcium deposits. Policosanol 
treatment therefore induced hypolipaemic, antioxidant, anti-inflammatory effect along with remarkable inhibiton of calcification in the aorta as compared to Atorvastatin.

This may express policosanol as a promising candidate for inhibition of vascular calcification and further clinical evaluations are requested in addition to its well-known potential as anti-diabetic, anti-inflammatory, antioxidant and hypolipaemic agent.

No conflict of interest 
174

HYPERTENSION AND THE HEART

\title{
HYPERTENSION AND STABLE CORONARY ARTERY DISEASE
}

\author{
R. Pedrinelli \\ Institute of Clinical Physiology, Pisa, Italy
}

Systemic hypertension is highly prevalent in stable coronary artery disease, a pervasive comorbidity complicating the diagnostic performance and interpretation of non-invasive provocative tests in chest pain patients because of the ischemic signals generated, despite normal or near normal coronary arteries, by hearts structurally readapted by long-term exposure to raised systemic blood pressure. Additional and unresolved problems posed by arterial hypertension in patients with stable coronary artery disease regard the benefits of antihypertensive treatment due to reports of irrelevant if not detrimental effect of BP lowering in averting coronary relapses as well as the lack of association between BP levels and incident coronary events in survivors from acute myocardial infarction. Uncertainties extend to blood pressure independent cardioprotective effects of antihypertensive drugs although the efficacy of renin-angiotensin system blockers in the long-term prevention of cardiovascular events in stable coronary artery disease patients has been shown by several studies, particularly when combined with amlodipine, a dihydropiridine calcium channel blocker. In contrast, the long-term effect of beta-blockers, the antihypertensive class most used in that clinical category, is not supported by strong evidence except that generated in patients with systolic dysfunction and early postmyocardial infarction recovery periods.

No conflict of interest 
177

\title{
INTERVENTIONAL CARDIOLOGY
}

\section{IMMEDIATE RESULTS AND MEDIUM TERM FOLLOW-UP OF TRANSCATHETER AORTIC VALVE IMPLANTATION IN VERY ELDERLY PATIENTS}

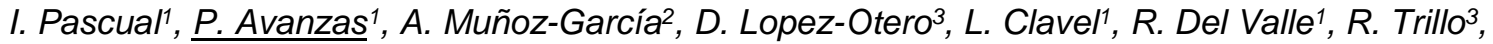 \\ J.M. Hernandez ${ }^{2}$, C. Morís ${ }^{1}$ \\ ${ }^{1}$ Hospital Central de Asturias, Cardiology, Oviedo, Spain \\ ${ }^{2}$ Hospital Universitario Virgen de la Victoria., Cardiology, Málaga, Spain \\ ${ }^{3}$ Complejo Hospitalario de Santiago, Complejo Hospitalario de Santiago, \\ Santiago de Complostela, Spain
}

Aims: Our aim was to evaluate immediate transcatheter aortic valve implantation (TAVI) results and medium-term follow-up in very elderly patients with severe and symptomatic aortic stenosis (AS).

Method: Multicenter, observational and prospective study carried out in 3 hospitals. We included consecutive very elderly (>85 years) patients with severe (AS) treated with TAVI. The primary endpoint was to evaluate death from any cause at 2 years.

Results: The study included 160 consecutive patients with a mean age of $87 \pm 2.1$ years [range, $85-94]$ and a mean logistic EuroSCORE of $18.8 \% \pm 11.2 \%$, with 57 (35,6\%) patients scoring $\geq 20 \%$. Procedural success rate was $97.5 \%$. 25 (15.6\%) patients had acute complications, being mayor bleeding the most frequent. Global mortality rate during hospitalization was $8.8 \%(n=14)$ and 30-day mortality rate was $10 \%(n=16)$. Median follow up period was $252.24 \pm 232.17$

days. During the follow-up period, 28 (17.5\%) patients died, 17 of them due to cardiac cause. Two year overall and cardiac survival estimated rates using the Kaplan-Meier method were $71 \%$ and $86.4 \%$ respectively. Cox proportional hazard regression showed that the variable EuroSCORE $\geq 20$ was the unique variable associated with overall mortality (figure). 


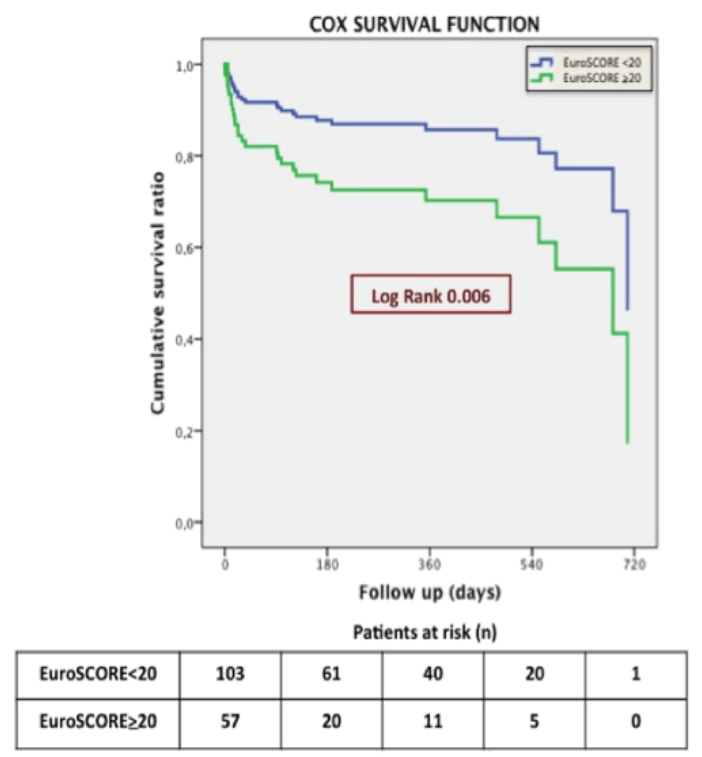

Conclusion: TAVI is safe and effective in a selected population of very elderly patients. Our findings support the adoption of this new procedure in this complex group of patients.

No conflict of interest 
178

\title{
INTERVENTIONAL CARDIOLOGY
}

\section{A NOVEL REPERFUSION FLUID WITH OXYGEN DELIVERY AND THERAPEUTIC PROPERTIES FOR PERCUTANEOUS CORONARY INTERVENTION (PCI)}

\author{
J. Simoni ${ }^{1}$, G. Simoni', J.F. Moeller ${ }^{2}$, P. Simoni ${ }^{3}$, D.E. Wesson ${ }^{4}$ \\ ${ }^{1}$ Texas HemoBioTherapeutics \& Biolnnovation Center- \\ Texas Tech University Health Sciences Center, Blood Substitutes \& Artificial Organs, Lubbock, \\ USA \\ ${ }^{2}$ Texas HemoBioTherapeutics \& Biolnnovation Center, Blood Substitutes \& Artificial Organs, \\ Lubbock, USA \\ ${ }^{3}$ Texas Tech University Health Sciences Center School of Pharmacy- \\ Covenant Healthcare System, Pharmacy Practice, Lubbock, USA \\ ${ }^{4}$ Texas A\&M Health Science Center College of Medicine- Baylor Scott \& White Health, \\ Internal Medicine, Temple, USA
}

\begin{abstract}
Aims: Although $\mathrm{PCl}$ is a life-saving technique; some patients experience thrombotic and restenotic complications. Recent studies suggest that the $\mathrm{PCI}$ procedure itself may cause endothelial (EC) trauma, which can trigger these events. To date, $\mathrm{PCI}$ reperfusion fluids are typically crystalloids without oxygen delivery capability or medicinal properties. Therefore, cardiologists worldwide are attempting more effective $\mathrm{PCl}$ reperfusion by concomitantly using nitric oxide (NO) donors or substrates, adenosine and/or adenosine receptor agonists. NO strategy, although appealing, has certain limitations, since it controls the vascular tone of the proximal portion of coronary artery (CA). Addition of adenosine is useful, as it dilates the distal portion of CA, has anti-platelet and EC cytoprotective effects; however, a very short half-life of only a few seconds limits its therapeutic usefulness.
\end{abstract}

Method: To address these limitations, we utilized a novel concept of pharmacologic cross-linking of hemoglobin $(\mathrm{Hb})$ molecule. Our PCl reperfusion fluid consists of $\mathrm{Hb}$ cross-linked with ATP and adenosine and conjugated with reduced glutathione (GSH). Scientific and medical rationale is based on modified $\mathrm{Hb}$ extended half-life, high oxygen delivery and the pharmacologic actions of its cross-linkers on CA.

Results: Pre-clinical studies revealed that this $\mathrm{PCI}$ reperfusion fluid can: (i) deliver oxygen, and has prolonged: (ii) vasodilatory activity via increasing production of CAMP, activating P2Y receptors and lowering NO decomposition rate, (iii) anti-platelet effect, (iv) GSH-driven antioxidative properties, (v) anti-inflammatory action via NF-kappa B stabilization, and (vi) cytoprotective effect on CA endothelium.

Conclusion: This $\mathrm{PCI}$ reperfusion fluid that is radiocontrast compatible, provides oxygen and sustained positive pharmacologic effects on CA.

No conflict of interest 


\title{
INTERVENTIONAL CARDIOLOGY
}

\section{PERCUTANEOUS AORTIC VALVE IMPLANTATION: CAN THE POSITIONING OF COREVALVE IN THE OUTFLOW OF THE LEFT VENTRICLE DECREASE THE INCIDENCE OF AV-BLOCK?}

\author{
M. De Oliveira \\ Rio De Janeiro, Brazil
}

The CoreValve's treatment presents lower mortality than replacement surgery for aortic stenosis in elderly patients.A prospective,single center,nonrandomized study evaluated if CoreValve's position can reduce the A-V's block incidence described from $37 \%$ to $50 \%$. The His bundle crosses the junction of the proximal and distal muscular septum. We measured the distance from aortic annulus until the end of the interventricular membranous septum using transthoracic ecocardiography preoperative and putting the Corevalve $2-3 \mathrm{~cm}$ under the aortic annulus avoiding injury in the His bundle. Method 26 symptomatics high-risk patients with an aortic valve area

Aims: The ventricular septal rupture (VSR) is a fetal complication of acute myocardial infarction (AMI), which was associated with a high mortality. Although percutaneous closure of VSR after AMI has emerged as an effective and alternative treatment of surgical repair, the optimal time and choice of treatment strategies were still controversial. We herein present a case of early VSR complicating AMI.

Method: A 72-year-old male presented with chest pain for 8 hours and electrocardiographic showed anterior wall AMI with a loud systolic murmur at the apex of heart. Echocardiography revealed a $14 \mathrm{~mm}$ VSR on the apical interventricular septum with left-to-right shunt (Figure 1). Emergent coronary angiogram was performed under the support of intra-aortic balloon pump, showing a total thrombus occlusion of the proximal left anterior descending (LAD) with normal right and left circumflex coronary artery.

Results: A drug-eluting stent was deployed to the LAD and the patient felt complete relief of chest pain after percutaneous coronary intervention. In case of VSR enlargement and high risk of closure at the early stage of edema and fibrosis after AMI, a percutaneous closure of VSR was performed 3 weeks later (Figure 2). After implantation of an Amplatzer device, echocardiography demonstrated a significant decrease of ventricular septal shunt. No major complications occurred. After 6-month follow-up, there was no angina or short breath in this patient and no residual shunt was detected. 


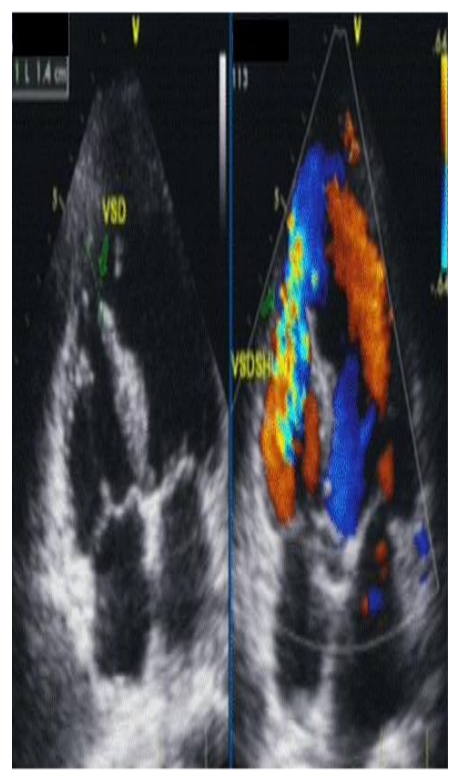

Figunel. Before Clossine

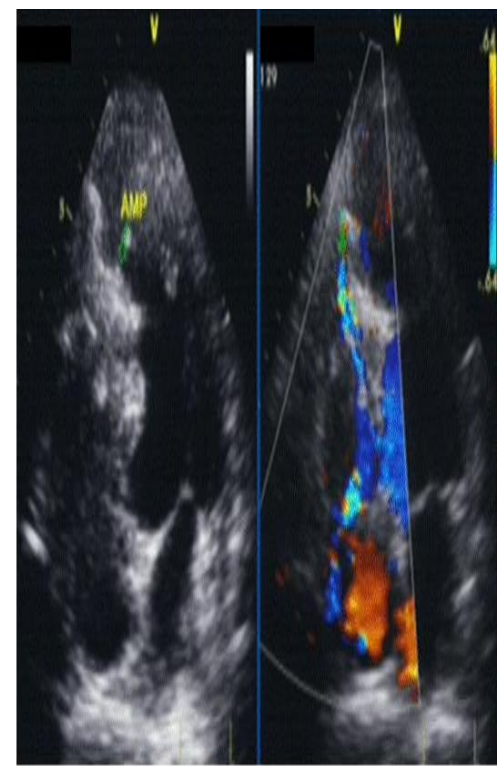

Figire. After Closile

Conclusion: Percutaneous closure of post-AMI VSR using Amplatzer device offers a safe and less invasive method. A late phase intervention after VSR diagnosis is advisable.

No conflict of interest 


\title{
INTERVENTIONAL CARDIOLOGY
}

\section{MINIMIZING NEGATIVE OUTCOMES OF CARDIOVASCULAR STENTING: ENDOTHELIALISATION OF STENT SURFACES}

\author{
N. Faridamin, T. Anson, A. Reynolds \\ Brunel University London, Department of Mechanical- Aerospace and Civil Engineering, London, \\ United Kingdom
}

Aims: Attracting vascular endothelial cells onto metal alloy stents has the potential to open a new avenue of therapy for small vessel stenting; included in this scenario are coronary artery vessels. It has been extensively documented in the scientific literature that these specialised vascular cells play leading role in maintaining vascular tone and stasis if continuous uninterrupted squamous endothelial cells line the lumen of a blood carrying vessels.

Method: The potential means of attracting vascular cells onto a synthetic object can be by applying a precise current that matches the cells of interest, but oppositely polarised. The essential principle of a negative being attracted to a positive is being exploited in this current research.

Results: Initial results using a bio mimic coronary artery system fitted with stents and appropriate physiological parameters, such as blood pressure, flow velocity, pulsatile signature and temperature; demonstrated the presence and adherence of vascular endothelial cells onto electrically polarised, bare metal stent surfaces in this case 316 stainless steel.

Conclusion: Despite of reductions in failure rates, there are still negative outcomes such as Instent restenosis, stent thrombosis after the stent deployment. This experiment has found that electrical charging can attract some types of cells as endothelial cells, including on the surface of the stent that prohibit thrombus formation, which is considered as a one of the main problems after coronary stenting.

No conflict of interest 
181

\section{INTERVENTIONAL CARDIOLOGY}

\section{CHRONIC TOTAL OCCLUSIONS PATIENTS \& LOW LEFT VENTRICULAR EJECTION FRACTION: INFLUENCE OF THE TREATMENT APPLIED ON THEIR PROGNOSIS}

V. Martin Yuste ${ }^{1}$, M. Cardona Olle', S. Prat' ${ }^{1}$ I. Ferrerira' ${ }^{2}$, M. Sabate ${ }^{1}$

${ }^{1}$ Hospital Clinic de Barcelona, Cardiology, Barcelona, Spain

2Hospital del valle de Hebron, Cardiology, Barcelona, Spain

Aims: Analyze the influence of treatment on the prognoses.

Method: Monocenter registry of consecutive CTO < LVEF patients admitted for angiography. Risks scores, clinical and angiographic characteristics were registered. Population was divided in 3 groups: I patients managed by medical therapy, II patients sent to CTO PCI and III patients sent to CABG. We compared the follow up between groups

Results: Between 2010 - 2012, 256 patients were registered. Age $65.5 \pm 11.7$ years, 16\% women, $71 \%$ hypertension, $43 \%$ diabetes, $64 \%$ smokers, previous AMI $46 \%, 78 \%$ multivessel disease. ACEF $2.4 \pm 0.77$, Syntax $25.2 \pm 11.5$. Treatment choice was left to the cardiologist's criteria. 157 patients assigned to Group I, 57 to Group II and 42 to Group III. Group I were older, had lower LVEF, worse renal function and stroke and hypertension more frequent. Group III had higher Syntax score. A PCI in non CTO vessel was performed in 34\% patients group I and $49 \%$ group II. Follow up (908.9 \pm 457 days) $10 \%$ patients from group I, $5,3 \%$ from group II and $2,4 \%$ from group III suffered AMI, $(p=0.133)$. 31\% patients from group I, $12 \%$ II y $7 \%$ III suffered from cardiac death $(p<0.001) .36 \%$ patients from group I, $14 \%$ in group II and III died from all causes of death $(\mathrm{p}<0.001)$.

Conclusion: Patients under medical therapy have a clinical profile that not justifies their poor prognosis. Patients sent to $C A B G$ had the lowest incidence of $A M I$ and cardiac death during the follow up

No conflict of interest 


\title{
MAJOR ADVERSE CARDIAC EVENTS FOLLOWING INTERVENTION ON THE CULPRIT VESSEL VERSUS ALL ATHEROSCLEROTIC VESSELS IN PATIENTS WHO PRESENTED WITH ST-ELEVATION MYOCARDIAL INFARCTION
}

\author{
A. Shafiee ${ }^{1}$, M. Salarifar 2 , M. Abbaszadeh ${ }^{3}$ \\ ${ }^{1}$ Tehran Heart Center - Tehran University of Medical Sciences, Cardiovascular Research, \\ Tehran, Iran \\ ${ }^{2}$ Tehran Heart Center- Tehran University of Medical Sciences, Interventional Cardiology, Tehran, \\ Iran \\ ${ }^{3}$ Tehran Heart Center- Tehran University of Medical Sciences, Cardiology, Tehran, Iran
}

\begin{abstract}
Aims: We aimed compared the rate of major adverse cardiac events (MACE) in multi vessel disease (MVD) patients who presented with ST elevation myocardial infarction (STEMI) and had percutaneous coronary intervention (PCl) performed on their culprit artery with those who underwent $\mathrm{PCl}$ on the culprit artery as well as other coronary branches.

Method: In this study, patients who were admitted to Tehran Heart Center with STEMI and underwent $\mathrm{PCl}$ between 2003 and 2011 were enrolled. Based on the coronary angiography results, patients were divided into single vessel disease and multi vessel disease. Patients with multi vessel disease were also categorized based on the $\mathrm{PCl}$ method: those had $\mathrm{PCl}$ on their culprit vessel only and those who had gradual $\mathrm{PCI}$ on other atherosclerotic vessels within 2 months as well as their culprit vessel. Then the 12-month MACE was compared between the study subgroups.
\end{abstract}

Results: In this study, 614 illegible patients were analyzed. Based on the coronary angiography results, 208 patients had single vessel disease, 204 patients had double vessel disease, and 202 patients had triple vessel disease. Total MACE in the multi-vessel incomplete PCI was significantly more than the other groups $(P=0.006)$. Moreover, the rate of cardiac death was significantly higher in the same group $(P=0.032)$. The frequency of $C A B G$ also tended to be higher in the culprit only PCl.

Conclusion: We concluded that patients who had PCI on all of their vessels had a better survival than those who had $\mathrm{PCl}$ on their culprit vessel alone.

No conflict of interest 
183

INTERVENTIONAL CARDIOLOGY

\title{
INTENTION-TO-TREAT PRIMARY PCI IN STEMI WITH FULL ST-SEGMENT RESOLUTION ON ADMISSION RESOLUTION SUBSTUDY
}

\author{
P. Kala, M. Holicka, J. Kanovsky, L. Koc, M. Mikolaskova, T. Novakova, P. Martin, I. Andrsova, \\ T. Novotny \\ University Hospital Brno and Masaryk University, Internal and Cardiology Department, Brno, \\ Czech Republic
}

Aims: To compare patients with STEMI and occluded vs. patent infarct-related artery (IRA) and no residual ST-segment elevation (full STR) on admission to cathlab.

Method: 20 patients ( $6.6 \%$ of total of 300 with average age $56.5 \pm 10.7$ years) with STEMI and full STR on admission were divided in Group A (6 patients with TIMI flow 0-1, i.e. occluded IRA) and Group B (14 patients with TIMI flow 2-3, i.e. patent IRA).

Results: Left anterior descending artery (LAD) represented only $16.6 \%$ of IRA in Group A in contrast to $28.6 \%$ in Group B. The left cirkumflex (LCX) territory represented $50 \%$ in Group A and only $21.5 \%$ in Group B and the right coronary artery (RCA) was found in $33.3 \%$ in Group A and $50 \%$ in Group B. Troponin value at $24 \mathrm{~h}$ from symptom onset was higher in Group A vs. B $(2.1 \pm 1.9$ vs. $1.2 \pm 1.5 \mathrm{ng} / \mathrm{ml} ; \mathrm{p}=0.55)$ though not statistically significant. None of the patients suffered repeated ST-elevation at the end of primary PCl.

Conclusion: The rate of patients with STEMI and full ST-resolution on admission is relatively low $(6.6 \%)$ and non-LAD territories represent majority of the infarct-related arteries (75\%). The intention-to-treat approach to primary $\mathrm{PCI}$ in these patients is safe and strongly supported by relatively high rate of occluded IRA that was found in $30 \%$.

Conflict of Interest Disclosure Statement: Supported by the Grant MH CZ NT13767-4 
184

INTERVENTIONAL CARDIOLOGY

\title{
PERCUTANEOUS CORONARY INTERVENTION IS A USEFUL BRIDGE TREATMENT FOR ACUTE MYOCARDIAL INFARCTION DUE TO ACUTE TYPE A AORTIC DISSECTION
}

\author{
S. Kodera \\ Asahi General Hospital, Cardiology, Asahi, Japan
}

\begin{abstract}
Aims: As the mortality rate of patients with acute myocardial infarction (AMI) with type A aortic dissection is high, treatment is challenging. The standard treatment is emergency surgery. However diagnosis is difficult in some cases, and the surgery may be difficult to perform. Some patients are so unstable that they cannot wait for surgery; for these patients percutaneous coronary intervention $(\mathrm{PCl})$ is useful. There are several reports of patients with $\mathrm{AMI}$ due to type $\mathrm{A}$ aortic dissection who were successfully treated with $\mathrm{PCl}$. We report a case of $\mathrm{AMI}$ due to type $\mathrm{A}$ aortic dissection that was successfully treated with $\mathrm{PCl}$ in an emergency situation.
\end{abstract}

Method: We report a 71-year-old woman with inferior AMI due to type A acute aortic dissection. Emergency enhanced computed tomography (CT) did not show obvious aortic dissection. During emergency percutaneous coronary intervention $(\mathrm{PCl})$, intravascular ultrasonography revealed type A aortic dissection. Hemodynamic stability was restored after PCl.

Results: 1 month later, CT revealed a sinus of Valsalva aneurysm, which was treated surgically.

Conclusion: This case suggests that $\mathrm{PCl}$ could be a good initial treatment option for unstable patients with AMI due to type A aortic dissection. This is the first reported case of sinus of Valsalva aneurysm subsequent to aortic dissection.

No conflict of interest 
185

INTERVENTIONAL CARDIOLOGY

\section{PERCUTANEOUS CLOSURE OF EARLY VENTRICULAR SEPTAL RUPTURE WITH ACUTE ANTERIOR WALL MYOCARDIAL INFARCTION}

R. Shi, L. Bai, P. Liu, Y. Wang, J. Ren, Y. Zhan', C. Sun

The First Affiliated Hospital of Xi'an Jiaotong University, Department of Cardiovascular Disease, Xi'an, China

Aims: The ventricular septal rupture (VSR) is a fetal complication of acute myocardial infarction (AMI), which was associated with a high mortality. Although percutaneous closure of VSR after AMI has emerged as an effective and alternative treatment of surgical repair, the optimal time and choice of treatment strategies were still controversial. We herein present a case of early VSR complicating AMI.

Method: A 72-year-old male presented with chest pain for 8 hours and electrocardiographic showed anterior wall AMI with a loud systolic murmur at the apex of heart. Echocardiography revealed a $14 \mathrm{~mm}$ VSR on the apical interventricular septum with left-to-right shunt (Figure 1). Emergent coronary angiogram was performed under the support of intra-aortic balloon pump, showing a total thrombus occlusion of the proximal left anterior descending (LAD) with normal right and left circumflex coronary artery.

Results: A drug-eluting stent was deployed to the LAD and the patient felt complete relief of chest pain after percutaneous coronary intervention. In case of VSR enlargement and high risk of closure at the early stage of edema and fibrosis after AMI, a percutaneous closure of VSR was performed 3 weeks later (Figure 2). After implantation of an Amplatzer device, echocardiography demonstrated a significant decrease of ventricular septal shunt. No major complications occurred. After 6-month follow-up, there was no angina or short breath in this patient and no residual shunt was detected.

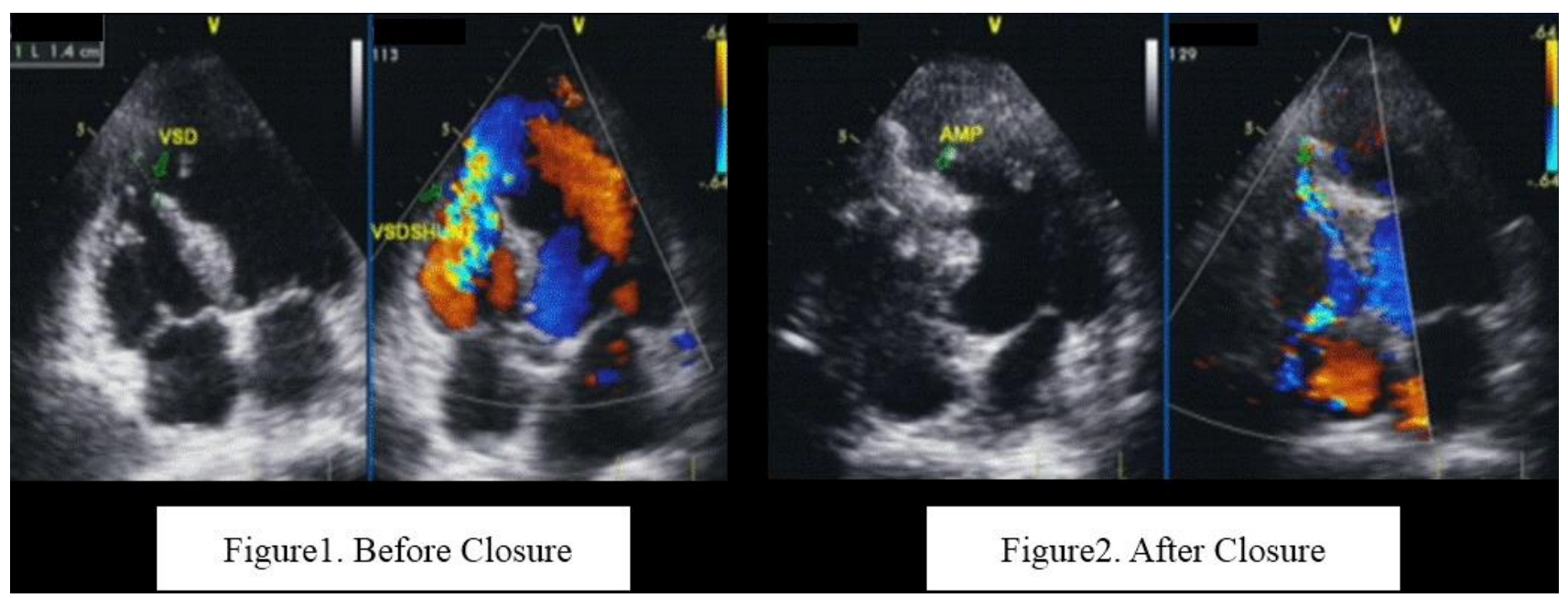

\section{Conclusion}

Percutaneous closure of post-AMI VSR using Amplatzer device offers a safe and less invasive method. A late phase intervention after VSR diagnosis is advisable. 
No conflict of interest

\title{
188
}

CLINICAL ASPECTS

STABLE ISCHEMIC HEART DISEASE: UPDATE 2015

\author{
U. Thadani \\ University of Oklahoma Health Sciences Center and Veterans Administration Medical Center, \\ Medicine- Cardiovascular Section, Oklahoma City- OK, USA
}

Aims: A variety of underlying pathophysiologic conditions may be responsible for stable ischemic heart disease (SIHD). Current treatment recommendations for SIHD are derived from outcome trials in symptomatic patients who had underlying coronary artery disease (CAD). The aim of treatment is 1) to reduce the incidence of serious adverse clinical outcomes (sudden cardiac death, myocardial infarction, heart failure and stroke); and 2) to improve quality of life by relieving or abolishing symptoms such as angina pectoris, shortness of breath and fatigue.

Method: Review of current literature.

Results: Treatment with anti-ischemic and anti-anginal medications and with coronary revascularization (percutaneous or bypass surgery) is invariably effective in relieving symptoms but has little impact on serious adverse clinical outcomes. While life style modification (cessation of smoking, regular exercise and diet); treatment with low dose aspirin and high dose potent statin , and control of blood pressure and diabetes reduce the incidence of serious clinical adverse outcomes, but do not improve symptoms. SIHD due to coronary artery spasm can be managed with a calcium channel blocker and or long acting nitrates. In 2015, treatment of patients with SIDH who have normal coronary arteries and evidence of impaired blood flow to the myocardium remains a challenge.

Conclusion: Many of the patients with SIHD are older, and have other comorbidities which must be taken into account when making treatment decisions. In patients with SIHD who have stable refractory angina, external enhanced counter pulsation may be effective; but cell therapy, and coronary sinus occlusion to relieve angina remain experimental.

Conflict of Interest Disclosure Statement:

Consultant to Gilead Science, speaker's bureau Amgenen and Gilead Science, and research grants for pharmaceutical studies to the institution. 
191

CLINICAL ASPECTS

THE ROLE OF NOVEL ANTI-DIABETIC AGENTS ON CARDIOVASCULAR RISK: FOCUS ON INCRETIN-BASED THERAPIES AND SGLT-2 INHIBITORS

\author{
M.M.R.I. Rizzo \\ DIBIMIS, School of Medicine, University of Palermo, Italy
}

The cardiovascular outcome represents a crucial issue for patients with type-2 diabetes. In the last years novel anti-diabetic agents have been developed, that are able to control glucose metabolism as well as to manage several cardiovascular risk factors, in order to ultimately impact the cardiovascular outcome. In this context, incretin-based therapies and SGLT-2 inhibitors are able to significantly manage glucose metabolism abnormalities, but they have also shown several beneficial non-glycemic effects, such as those on body weight, blood pressure, plasma lipids and markers of inflammation. The first data on the cardiovascular outcome trials with the use of these molecules are giving us a better picture on the role of these novel anti-diabetic agents on cardiovascular risk.

No conflict of interest 
192

CLINICAL ASPECTS

THE RATE OF ARTERIAL DIAMETER CHANGE: A NEW MEASURE OF ENDOTHELIAL FUNCTION DERIVED FROM TRADITIONAL MEASURES OF FLOW-MEDIATED DILATATION

\author{
J.Parker \\ Lunenfeld-Tanenbaum Research Institute Division of Cardiology, \\ Department of Medicine Mount Sinai and University Health Network Hospitals, Toronto, Ontario, \\ Canada
}

Background: Measures of flow-mediated dilatation (FMD) are used in the assessment of endothelial function. Traditionally, this is a single, time-based measure of maximal conduit artery dilatation. Little attention has been given to more integrated measures of the flow-mediated change in vessel diameter

Purpose:To examine the rate of change in radial artery diameter $(\mathrm{dD} / \mathrm{dt})$ after the ischemic stimulus used in the assessment of FMD.

Methods: We examined the peak rate of radial artery diameter change following the FMD stimulus. A total of 225 patients (62 \pm 5 yrs, 167 males) undergoing coronary angiography for known or suspected coronary artery disease and 49 normal volunteers ( $24 \pm 3$ yrs, 91 males) were studied.

Results: Although FMD was significantly blunted in the patient group as compared to the normal volunteers $(4.1 \pm 3.7$ vs $8.3 \pm 3.3 \%$; $P<0.001)$, the peak rate of radial diameter increase was significantly greater in the patient group as compared to the normal volunteers $(0.025$ vs 0.015 $\mathrm{mm} / \mathrm{sec} ; \mathrm{P}<0.001$; figures 1 and 2). The hyperemic/flow response was similar in both groups. There was a positive correlation between peak dD/dt max and both age and FMD $(P<0.001$ for both). There was no correlation between peak $\mathrm{dD} / \mathrm{dt}$ and baseline or maximum vessel diameter, peak hyperemic flow or with flow mediated constriction (FMC).

Conclusions: Therefore, in comparison to a group of normal volunteers during the measurement of FMD there are important differences in the rate of radial artery diameter change. The mechanism of the greater rate of arterial dilatation remains uncertain, however this measurement may provide further insight into vascular function as it assessed by FMD. 


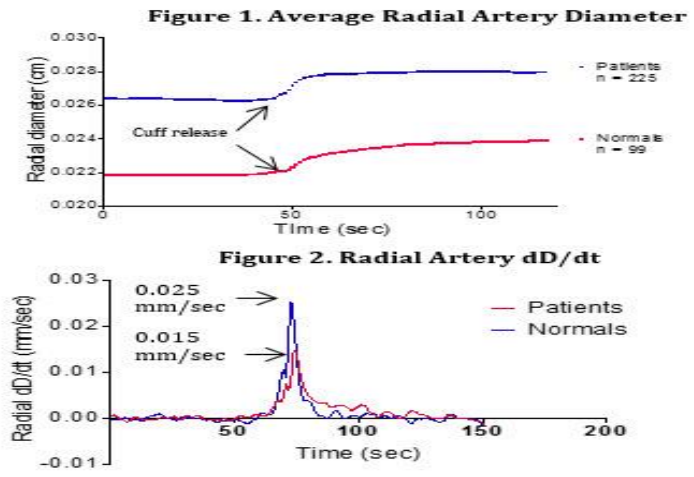

No conflict of interest 


\title{
TREATMENT GAPS IN MANAGAMENT OF PATIENTS FOLLOWING ACUTE MYOCARDIAL INFARCTION: ANALYSIS OF REAL-WORLD DATA
}

\author{
D. Weitzman ${ }^{1,2}$, L. Bash ${ }^{3}$, O. Sharon ${ }^{4}$, A. Milwidsky ${ }^{5}$, G. Chodick ${ }^{1,2}$, V. Shalev ${ }^{1,2}$ \\ ${ }^{1}$ Maccabi Healthcare Services, Maccabitech, Tel Aviv, Israel \\ ${ }^{2}$ Tel-Aviv University, Sackler Faculty of Medicine, Tel Aviv, Israel \\ ${ }^{3}$ Merck \& Company- Inc., Outcomes Research- C.O.R.E. PC/Cardio, Rahway, USA \\ ${ }^{4} \mathrm{MSD}$ Israel, Medical director, Hod Hasharon, Israel \\ ${ }^{5}$ Tel Aviv Sourasky Medical Center, Internal Department E, Tel Aviv, Israel
}

\begin{abstract}
Aims: To characterize long-term management of patients who experience an MI using 'realworld' data.
\end{abstract}

Method: Patients who experienced a myocardial infarction (MI) between 01/2009 and 12/2010 were identified among adult members of a large health organization in Israel (Maccabi Healthcare Services). Demographic, clinical, pharmacy and laboratory data were derived using electronic medical records. Patients with a history of CVA or TIA were excluded from the study to focus on those whose antiplatelet treatment wouldn't substantially be influenced by bleeding risk.

Results: A total of 1649 eligible incident MI patients were identified (75\% men, mean age $=64.7 \pm 14.2 \mathrm{y})$. Among 1521 patients surviving $>60$ days from hospital discharge, $8.5 \%$ and $5.7 \%$ of patients remained untreated at 30 and 60 days post $\mathrm{Ml}(17.8 \%$ and $13.0 \%$ of women and $5.4 \%$ and $3.2 \%$ of men, respectively, $\mathrm{P}<0.001)$. Men were most often $(58 \%)$ treated with dual antiplatelet therapy (DAPT, clopidogrel + aspirin), while women were most often treated with aspirin alone $(41 \%)$, and next on DAPT $(38 \%)$. A considerable proportion of patients $(10 \%$ in men and $26 \%$ in women) have not been seen by a cardiologist during the year following the MI.

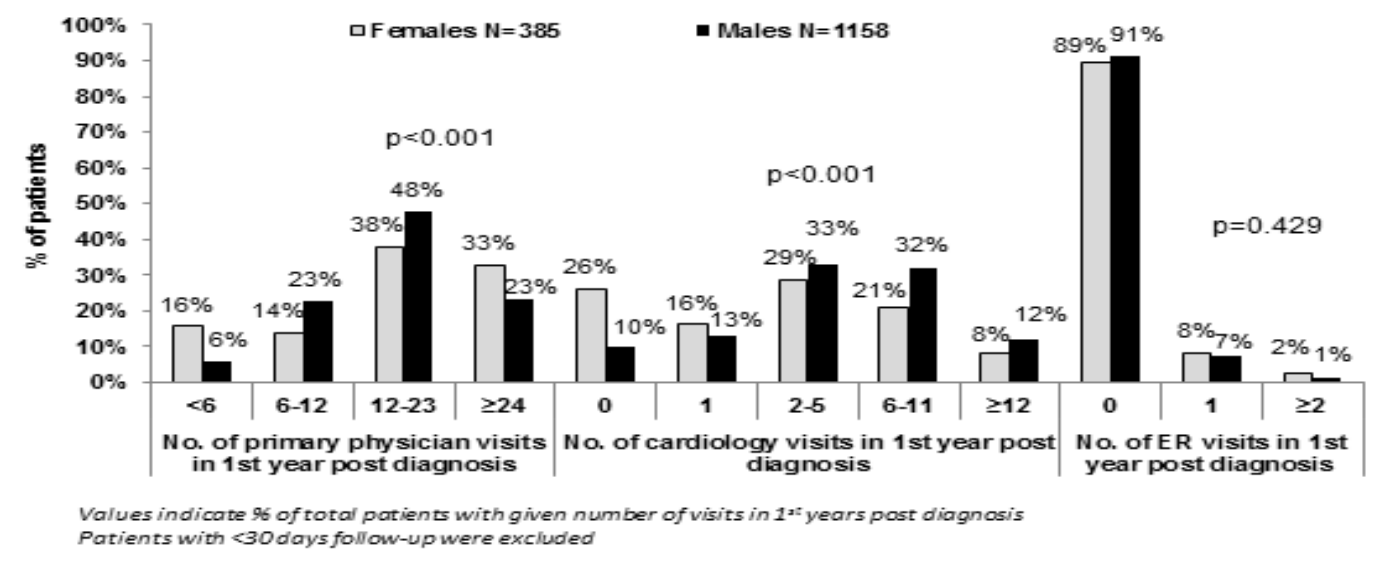


Conclusion: A considerable proportion of post-MI patients go untreated with antiplatelet therapy and without seeing a cardiologist, particularly among women. Further efforts are required to assess how gender may drive disease management and subsequently prognosis among these patients.

Conflict of Interest Disclosure Statement:

The study was funded by Merck \& Company, Inc. 


\title{
197 \\ PROGNOSIS, PROGRESSION, PRACTICE PATTERNS \\ DOCTOR-PATIENT COMMUNICATION ON NUTRITION AND THE RELATED SECONDARY PREVENTIVE BEHAVIOURS
}

\author{
M. Baumann ${ }^{1}$, E. Le Bihan ${ }^{1}$, N. Lorentz ${ }^{2}$, A. Tchicaya ${ }^{2}$ \\ ${ }^{1}$ University of Luxembourg, Research Unit INSIDE- Institute Health \& Behaviour, Esch-sur- \\ Alzette, Luxembourg \\ ${ }^{2}$ Luxembourg Institute of Socio-Economic Research, Living Conditions, Esch-sur-Alzette, \\ Luxembourg
}

Aims: Doctor-patient relationship impacts on the compliance. For hypercholesterolemia, diabetes, overweight and obesity, our study's aim was to analyse the associations between the quality of communication, evaluated in 2013, and patients' adherence to their general practitioner (GP)'s nutritional advice, between 2008 and 2013.

Method: Five years after a coronary angiography performed by the National Institute of Cardiac Surgery and Interventional Cardiology in Luxembourg, 4391 patients were contacted. 1289 completed a self-rating questionnaire assessing the GP'Com-5 items scale (Cronbach 0.87 ). The variables were analysed with a multiple logistic regression model.

Results: Patients declared that they reduced or stopped their consumption $71.9 \%$ of fat, $62.8 \%$ of sugar and $65 \%$ increased their consumption of fruits and vegetables. Around $37 \%$ of the patients reported having made changes to their risk behaviours following the advice of their doctor. An increased consumption of fruits and vegetables was linked with the quality of doctorpatient communication when patients were overweight (odd ratio $=1.081$, IC 95\%, [1.013-1.155]), obesity $(\mathrm{OR}=1.130$ [1.056-1.209]), hypercholesterolemia $(\mathrm{OR}=1.102[1.033-1.175])$ and diabetes $(\mathrm{OR}=1.103$ [1.029-1.181]). The reduction or cessation of sugar consumption was associated with the quality of physician-patient communication when the patient was overweight $(\mathrm{OR}=1.093$ [1.020-1.170]), and more so obese $(\mathrm{OR}=1.106$ [1.031-1.187]). The same held true for patients with hypercholesterolemia $(\mathrm{OR}=1.103$ [1.031-1.180]) or diabetes $(\mathrm{OR}=1.173$ [1.088-1.265]).

Conclusion: In prevention of secondary CVDs, doctor-patient communication is a relevant determinant in obtaining a favourable behavioural outcome, especially with the consumption of fresh fruit and vegetables.

No conflict of interest 


\section{8 \\ PROGNOSIS, PROGRESSION, PRACTICE PATTERNS \\ EFFECT OF CARDIAC REHABILITATION FOLLOWING ACUTE CORONARY SYNDROME ON LONG TERM OUTCOMES}

S. Klainman ${ }^{1,2}$, A. Yarmolovsky ${ }^{1}$, G. Fink ${ }^{1}$

${ }^{1}$ Exercise Physiology Unit Kaplan MC Rehovot, Israel

${ }^{2}$ Gefen Institute for Research and Cardiac Health, Bat-Yam, Israel

Background: The benefit of cardiac rehabilitation on ischemic patients is well known, especially in the short term post the rehabilitation program, and less in the long term of outcomes.

Aim of study: To compare the outcomes of post ACS patients with versus without performing a cardiac rehabilitation program following the hospitalization, within 4-6 years of follow-up.

Methods: 156 Patients who underwent ACS between years 2006-2008, were followed. 80 had performed a cardiac rehabilitation program of 3-6 months following the acute event (Group A), while the rest of patients (Group B; $n=76$ ) had not. All patients were followed for 4-6 years post the ACS for outcomes including cardiac and non-cardiac hospitalizations, PTCAs, CABG, CHF development, cardiac arrhythmias, renal failure and death.

Results: The follow-up results were as follows (Group A vs. Group B, respectively): Age $-60 y+/-$ 10.5 vs. $64+/-11, P=$ ns; Number of cardiac hospitalizations -61 vs. 129 ; Number of non-cardiac hospitalizations -36 vs. 119 ; PTCA intervention - 10 vs. 14; CABG - 2 vs. 3; CHF development -5 vs. 6 ; Cardiac arrhythmias -2 vs. 8 ; Renal failure -3 vs. 4 ; and Death -1 vs. 9

Conclusions: The results clearly indicate that post ACS patients who performed a rehabilitation program following the event demonstrated a better outcome, cardiac and non-cardiac, than those without rehabilitation, within a long term follow-up period of 4-6 years. A possible explanation might be that patients who participate in rehabilitation programs are more aware of healthy lifestyle in general, which might reduce such outcomes even in the long term of follow-up.

Document not received 
199

PROGNOSIS, PROGRESSION, PRACTICE PATTERNS

\section{MID-TERM PROGNOSIS OF PATIENTS WITH NON-ST ELEVATION ACUTE CORONARY SYNDROME AND NON-SIGNIFICANT CORONARY ARTERY DISEASE}

T. Lozano' ${ }^{1}$ J.M. Ruiz-Nodar ${ }^{1}$, A. Cequier-Fillat ${ }^{2}$, C. Morís de la Tassa ${ }^{3}$

${ }^{1}$ Hospital General Universitario, Cardiology Department, Alicante, Spain

${ }^{2}$ Hospital Universitario de Bellvitge, Cardiology Department, L' Hospitalet de Llobregat Barcelona, Spain

${ }^{3}$ Hospital Universitario Central de Asturias, Cardiology Department, Oviedo, Spain

Aims: We assessed the mid-term prognosis of a cohort of patients enrolled in a multicentric registry hospitalized for non-ST-segment elevation acute coronary syndrome (NSTEACS) and non-significant coronary artery disease (NSCAD).

Method: A total of 1133 patients were included. Of these, $687(60.6 \%)$ underwent angiography. Patients were divided in four groups: $102(14.8 \%)$ had NSCAD (group 1), $222(32.3 \%)$ one vessel disease (group 2), 176 (25.6\%) two vessel disease (group 3), and 187 (27.2\%) three vessel or left main disease (group 4). We assessed clinical characteristics, total mortality and major adverse cardiovascular events at 12 month follow-up.

Results: Patients of group 1 (NSCAD) were younger than the population with obstructive disease $(64,8 \pm 10,6$ vs $67,4 \pm 11,4$ years, $p=0,034)$. We did not find differences in the prevalence of cardiovascular risk factors. At discharge, secondary prevention was less optimal in patients with NSCAD: ASA (72 vs 93,8\%, $p<0,001$ ), clopidogrel (32 vs 84,8\%, $p<0,001$ ), betablockers (45 vs 78,7\%, $p<0,001$ ), ACE inhibitors (30 vs 44,3\%, $p=0,008$ ) and statins (62 vs $88,7 \%, p<0,001)$. One year mortality for groups $1,2,3$ y 4 was respectively $2,1 \%, 2,4 \%, 6 \%$ y $10,7 \%(p=0,001)$ and MACE were respectively 2,1\%, 9,9\%, 11,4\% y $21,3 \%(p<0,001)$.

\section{Conclusion:}

Patients admitted for NSTEACS and NSCAD show similar one-year mortality than those with one-vessel disease. Their prognosis with respect to MACE is better than the population with obstructive disease, despite a less intensive secondary prevention strategy.

No conflict of interest 
200

PROGNOSIS, PROGRESSION, PRACTICE PATTERNS

\section{PERIOPERATIVE CLOPIDOGREL IS SAFE IN MAJOR ARTERIAL SURGERY}

C. Saadeh

Lebanese University, Vascular Surgery, Beirut, Lebanon

Aims: The purpose of this prospective, non-randomized, comparative study was to determine the impact of preoperative exposure to clopidogrel and aspirin on perioperative bleeding complications, in patients undergoing open major arterial surgery.

Method: 1045 consecutive major arterial procedures, in the last 10 years, were classified into two groups: the Clopidogrel group consisted of 482 procedures performed under dual clopidogrel and aspirin up to the time of surgery; while the No Clopidogrel group consisted of 563 procedures completed on patients who were taking neither medication or were on aspirin alone. The primary composite end point was reoperation for bleeding and bleeding-related death. Secondary endpoints included blood transfusion requirements, hematoma formation, duration of the procedures as well as length of stay.

Results: The first phase of the study, reporting on 647 arterial procedures by early 2012, was published in the $J$ Vasc Surg 2013;58:1586-92. Patients on clopidogrel had a higher cardiovascular risk profile, and a higher prevalence of prior peripheral and coronary stents $(\mathrm{P}<.0001)$. Clopidogrel use $(46 \%)$ was common across all operation categories. There was no statistically significant difference in the primary end point between the two groups $(0.8 \%$ and $0.5 \%$ respectively, $\mathrm{P}=\mathrm{NS}$ ). Blood transfusions were mainly required by patients undergoing aortic surgery, at similar rates and volumes in both groups. The mean operative time and ICU/hospital lengths of stay were not longer in clopidogrel recipients.

Conclusion: Combined therapy with clopidogrel and aspirin up to the day of surgery is not associated with increased bleeding complications or transfusion requirements in patients undergoing peripheral arterial surgery.

No conflict of interest 


\title{
201
}

\section{PROGNOSIS, PROGRESSION, PRACTICE PATTERNS}

\section{CLINICAL CHARACTERISTICS, MANAGEMENT, AND 1-YEAR OUTCOMES OF PATIENTS WITH ACS IN IRAN: THE IRANIAN PROJECT FOR ASSESSMENT OF CORONARY EVENTS2 (IPACE2)}

\author{
F. Masoudkabir' $^{1}$ S.E. Kassaian², H. Sezavar ${ }^{3}$, S.E. Alavinia ${ }^{4}$, A. Pourmoghaddas ${ }^{5}$, J. Kojouri6, \\ S. Ghaffari ${ }^{7}$, F. Alaeddini ${ }^{8}$, E. Mir ${ }^{9}$ \\ ${ }^{1}$ Tehran Heart Center- Tehran University of Medical Sciences, Cardiology, Tehran, Iran \\ ${ }^{2}$ Tehran Heart Center- Tehran University of Medical Sciences, Department of Cardiology, Tehran, \\ Iran \\ ${ }^{3}$ Rasul-e-Akram Hospital- Iran University of Medical Sciences, Department of Cardiology, Tehran, \\ Iran \\ ${ }^{4}$ Javadolaemmeh Hospital- Mashhad University of Medical Sciences, Department of Cardiology, \\ Mashhad, Iran \\ ${ }^{5}$ Khorshid Hospital- Isfahan University of Medical Sciences, Department of Cardiology, Isfahan, \\ Iran \\ ${ }^{6}$ Shiraz University of Medical Sciences, Cardiovascular Research Center, Shiraz, Iran \\ ${ }^{7}$ Tabriz University of Medical Sciences, Cardiovascular Research Center, Tabriz, Iran \\ ${ }^{8}$ Tehran Heart Center- Tehran University of Medical Sciences, Department of Research, Tehran, \\ Iran \\ ${ }^{9}$ Sanofi Iran, Medical Operations Department, Tehran, Iran
}

Aims: To assess contemporary data on characteristics, management and 1-year post-discharge outcomes in Iranian patients hospitalized with acute coronary syndrome (ACS).

Method: Between April 2011 and November 2012, we established a prospective multicenter registry that recruited patients with confirmed diagnosis of ACS that discharged alive from 11 tertiary care hospitals in 5 major cities of I.R. Iran. Data on clinical characteristics and in-hospital management of the patients were collected and patients were followed-up regarding the use of medications and the endpoints of the study at 1-month and 1-year after discharge. The primary end point of this study was major adverse cardiovascular events (MACCE), defined as mortality, ACS, and stroke/TIA.

Results: A total of 1799 patients (25.7\% STEMI and 74.3\% UA/NSTEMI) that discharged alive with confirmed diagnosis of ACS were included in final analysis. During hospitalization, the majority of the patients received aspirin (98.6\%), clopidogrel(91.8\%), anticoagulants $(93.4 \%)$, statins $(94.3 \%)$ and beta-blockers(89.3\%). Reperfusion therapy was done in $62.6 \%$ of STEMI patients (46.3\% thrombolytic therapy and $17.3 \%$ primary PCI). The mean door-to-balloon and door-to-needle times were 82.9 and 45.6 minutes, respectively. In our study, $64.7 \%$ and $79.5 \%$ of the patients in UA/NSTEMI and STEMI groups, respectively, underwent coronary angiography. During the 12-months after discharge, MACCE occurred in $15.0 \%$ of all patients.

Conclusion: Our study showed that composition of Iranian ACS patients regarding the type of ACS is similar to developed European countries and is unlike developing countries of the middleEast and Africa. We found that our ACS patients are treated with high levels of adherence to guideline-recommended in-hospital medications. 
No conflict of interest 
202

\title{
PROGNOSIS, PROGRESSION, PRACTICE PATTERNS
}

\section{PREDICTION OF POSTOPERATIVE LOW CARDIAC OUTPUT SYNDROME WITH NOVEL CARDIAC BIOMARKER}

\author{
V. Shumavets ${ }^{1}$, M. Kaljadka ${ }^{2}$, I. Andraloits' ${ }^{1}$, I. Russkih ${ }^{2}$, M. Bushkevich ${ }^{1}$, Y. Ostrovsky ${ }^{1}$ \\ ${ }^{1}$ Belarus Cardiology Centre, Cardiac Surgery, Minsk, Belarus \\ ${ }^{2}$ Belarus Cardiology Centre, Clinical laboratory, Minsk, Belarus
}

Aims: Our study focused on prognostic capacity of cardiac biomarker in patients with severe compromised left ventricle (LV) to predict low cardiac output syndrome (LCOS) after open heart surgery.

Method: 73 patients with severe depressed LV function (EF $<35 \%$ ) were included in pilot prospective study. Patients underwent either combined CABG with mitral-valve procedure (49 patients) or isolated mitral-valve repair or chordal-sparing replacement (24 patients) consequently. Blood samples for measurements of cardiac biomarkers (sST2, NT-proBNP, hscTnl and CRP) were collected preoperatively. The primary end point was complicated postoperative period due to LCOS (duration of isotopes more then $24 \mathrm{~h}$, intra-aortic balloon pump using, temporary VAD application or hospital mortality).

Results: Cardiac-related complications were observed in 27 patients (37\% of cases). Preoperatively only level of SST2 was significantly higher in patients with cardiac-related complications during hospital stay $(86,9(49,4-113,1)$ vs. $25,3(19,8-35,8)$ respectively, $p=0,001)$. While no difference were found in NT-proBNP (2000 (427-6577) vs. 1200 (870-2169), $p=0,422)$ between patients with complicated or not postoperative period. AUC was also highest for preoperative sST2 level $-0,852(95 \% \mathrm{Cl} 0,691-1,014, p=0,02)$. The best cut-off value of the preoperative sST2 level was $45 \mathrm{ng} / \mathrm{ml}$ showed a sensitivity of $81,81 \%$ and specificity of $93,75 \%$ in predicting the complicated postoperative period. A sST2 level higher $45 \mathrm{ng} / \mathrm{ml}$ was identified as predictors for cardiac-related complication after open heart surgery $(\mathrm{OR}-5,345(95 \% \mathrm{Cl} 3,6$ $9,78, p=0,01)$.

Conclusion: Preoperative level of SST2 can be used to identify patients at increased risk of LCOS.

No conflict of interest 
203

\title{
PROGNOSIS, PROGRESSION, PRACTICE PATTERNS
}

\section{EFFECT OF SHORT TERM SUPERVISED PHASE II CARDIAC REHABILITATION ON ENDOTHELIAL FUNCTION USING FLOW MEDIATED DILATION IN CORONARY ARTERY DISEASE PATIENTS}

 \\ T. Aprami ${ }^{1}$ \\ ${ }^{1}$ Padjadjaran University, Department Cardiology and Vascular Medicine, Bandung, Indonesia \\ ${ }^{2}$ Cibabat Hospital, General medicine, Bandung, Indonesia \\ ${ }^{3}$ Ujung Berung Hospital, Department Cardiology and Vascular Medicine, Bandung, Indonesia \\ ${ }^{4}$ Padjadjaran University, Department of Physical and Rehabilitation Medicine, Bandung, \\ Indonesia
}

\begin{abstract}
Aims: Exercise based cardiac rehabilitation $(\mathrm{CR})$ is associated with an improvement in endothelial function. We aim to investigate the effect of short term phase II CR on endothelial function in patients with coronary artery disease (CAD).
\end{abstract}

Method: The inclusion criteria were CAD patients participated in the phase II CR at Dr. Hasan Sadikin Hospital from October 2014 to April 2015. The program lasts 4-8 weeks, consist of 6-12 education, aerobic exercise, moderate intensity and continuous training, minimum once weekly, for 30 minutes each. Flow mediated dilation (FMD) examination on brachial artery was done at baseline and upon completion of the CR.

Results: The study included 38 patients, 2 patients were excluded because could not fulfill the study criteria. Ex-smoker as the most common risk factor was found in $77.8 \%$ patients, $66.7 \%$ patients had dyslipidemia, $52.8 \%$ patients with hypertension and $11.1 \%$ patients with diabetes. Although not significant, FMD tended to increase after CR from $7.8 \pm 0.8 \mathrm{~mm}$ to $10.2 \pm 1.0 \mathrm{~mm}$ $(p=0.062)$. Analysis of dyslipidemia and hypertensive patients showed significant improvement in FMD $(6.7 \pm 0.8 \mathrm{~mm}$ to $10.3 \pm 1.4 \mathrm{~mm}, \mathrm{p}=0.038)$ and $(7.6 \pm 1.3 \mathrm{~mm}$ to $11.9 \pm 1.6 \mathrm{~mm}, \mathrm{p}=0.049)$. FMD improvement were not significant in diabetes $(8.5 \pm 2.6 \mathrm{~mm}$ to $13.6 \pm 1.7 \mathrm{~mm}, p=0.121)$ and ex-smoker patients $(7.2 \pm 0.8 \mathrm{~mm}$ to $9.1 \pm 1.1 \mathrm{~mm}, \mathrm{p}=0.146)$.

Conclusion: Short term exercise based $\mathrm{CR}$ associated with an improvement in endothelial function in CAD patients especially with dyslipidemia and hypertension as a risk factor.

No conflict of interest 


\section{CARDIAC SURGERY: CURRENT ISSUES}

\section{CARDIAC SURGICAL TRAINING IS SAFE FOR PATIENTS}

\section{E. Caruana, S. Nashef}

Papworth Hospital NHS Foundation Trust, Cardiothoracic Surgery, Cambridge, United Kingdom

Aims: We sought to evaluate the postoperative results of patients operated upon by trainees as the primary surgeon, at a single cardiac surgical centre in the United Kingdom.

Method: Prospectively collected data were obtained for all patients who had cardiac surgery at our institution between January and December 2013. Patients operated by trainees were identified, and compared with propensity-matched control patients operated by consultant surgeons. Student's t-test and Pearson's Chi-squared test were used for statistical analyses.

Results: During the study period, trainees performed, under direct consultant supervision, 482 surgical procedures of which $135(28.0 \%)$ were urgent or emergent. There was no difference between trainee-led and consultant-led cases in logistic EuroSCORE $(7.96 \pm 9.44$ vs $7.72 \pm 9.52$, p $=0.691)$, operative time (227.1 \pm 64.8 vs $222.5 \pm 68.9$ minutes, $p=0.284)$, bypass time $(95.1 \pm 42.9$ vs $89.7 \pm 45.1$ minutes, $p=0.055)$, and aortic cross-clamp time (60.6 \pm 28.7 vs $57.0 \pm 32.8$ minutes, $\mathrm{p}=0.072)$; hospital mortality (1.2 vs $2.3 \%, \mathrm{p}=0.221)$ and 90 -day mortality $(2.5$ vs $3.7 \%, p=$ $0.266)$ mortality. Those patients operated on by trainees had a slightly shorter length of stay $(9.2 \pm 5.2$ vs $10.7 \pm 9.4$ days, $p=0.0023)$.

Conclusion: There is no demonstrable increase in operative times, morbidity or mortality associated with trainee-led cardiac surgical procedures performed under appropriate consultant supervision.

No conflict of interest 
207

CARDIAC SURGERY: CURRENT ISSUES

\title{
LONG-TERM EFFECT OF DUAL ANTIPLATELET TREATMENT AFTER CORONARY ARTERY BYPASS GRAFTING IN DIABETIC PATIENTS
}

\author{
J. Lopez-Menendez'1, F. Callejo², P. Avanzas', C. Morales², J.C. Llosa², B. Meana', J. Silva² \\ ${ }^{1}$ Hospital Ramón y Cajal, Cardiac Surgery, Madrid, Spain \\ ${ }^{2}$ Hospital Universitario Central de Asturias, Cardiac Surgery, Oviedo, Spain \\ ${ }^{3}$ Hospital Universitario Central de Asturias, Cardiology, Oviedo, Spain
}

\begin{abstract}
Aims: Diabetic patients have an increased incidence of late major adverse cardiovascular events (MACE) after coronary artery bypass surgery (CABG), and platelet aggregation is related to the appearance of MACE.

We analyzed the effect of dual antiplatelet treatment (DAT), compared to single antiplatelet treatment (SAT), over late outcomes after CABG in a diabetic population.
\end{abstract}

Method: Retrospective study that included all diabetic patients discharged after CABG, during the years 2009 and 2010, with a two years clinical follow-up.

Antiplatelet treatment was initiated before discharge, and its effect on patients' prognosis was analyzed. The primary end-point was the composite of acute coronary syndrome, unplanned revascularization, stroke and cardiovascular death.

Results: The study included 187 diabetic patients. 15.5\% had a primary end-point event during follow-up (9.4\% in non-diabetic patients). Insulin dependent diabetics (IDD) had a higher event rate $(19.9 \%)$ than non-insulin dependent diabetics (NIDD) (14.2\%).

The study group was divided into: A) DAT 70 patients (37.4\%); B) SAT 117 patients (62.6\%).

DAT was associated with a significant decrease of MACE (DAT $7.1 \%$ vs. SAT $20.5 \%$; $p=0.023$ ).

Freedom from MACE was analyzed using Cox Proportional Hazards regression. DAT was

associated with a significant reduction in event rate (Hazard ratio 0.326; 95\%IC $0.124-0.854$;

$p=0.023$ ). The beneficial effect of DAT was higher in IDD (Hazard ratio $0.180 ; 95 \%$ IC 0.023 -

1.443).

Conclusion: Dual antiplatelet treatment in diabetic patients is associated with a reduction of late MACE after CABG.

No conflict of interest 


\title{
CARDIAC SURGERY: CURRENT ISSUES
}

\section{TWENTY-YEAR RESULTS OF AORTIC ROOT SURGERY IN PATIENTS AFFECTED BY MARFAN SYNDROME: A SINGLE-CENTER FOLLOW-UP EXPERIENCE}

\author{
P. Nardi, F. Nicolò, E. Bovio, A. Lio, A. Scafuri, A. Pellegrino, G. Ruvolo \\ Cardiac Surgery Unit and Marfan Center, Tor Vergata University Policlinic, Rome, Italy
}

Objectives: The aim of this study is to compare long-term results of patients affected by Marfan Syndrome (MFS) undergoing aortic root replacement with a valved conduit (Bentall operation) or aortic valve-sparing reimplantation (David operation) for the treatment of the aortic root dilatation.

Methods: Out of one-hundred and fifty MFS patients followed at our Marfan center since 2008, 67 patients underwent surgery in our Institution (70\%) or in other cardiac centers since 1994. Thirty patients underwent Bentall operation (Bentall Group), 29 underwent David operation (David Group); other surgical procedures were performed in 8 patients.

Results: No operative mortality was recorded. At 20 years (mean follow-up 97 \pm 82 , range 1-369, months), no prosthesis-related major bleeding or thromboembolic events, were observed; 20-year survival was $94 \pm 6 \%$ in Bentall Group and $100 \%$ in David Group, respectively ( $P=0.32)$, freedom from reintervention for aortic valve dysfunction was $100 \%$ in the Bentall Group and $75 \pm 13 \%$ in the David Group, respectively $(P=0.04)$; the difference became significantly relevant after the first 8 year period of follow-up. Freedom from all-cause death, myocardial infarction, stroke, prosthetic valve-related complications, reintervention on any aortic segment including aortic valve replacement, was $69 \pm 12 \%$ in Bentall Group and $67 \pm 14 \%$ in David Group( $P=0.33)$.

Conclusions: Both surgical techniques are good options for MFS patients and confer similar satisfactory results during a long-term follow-up period, but the risk for reintervention of the David operation is higher in comparison with Bentall operation after a mid-tem follow-up period.

Therefore, Bentall operation in MFS patients continues to be the gold standard procedure in this subset of patient population.

No conflict of interest 


\title{
CARDIAC SURGERY: CURRENT ISSUES
}

\section{CORONARY ARTERY RECONSTRUCTION WITH THE INTERNAL THORACIC ARTERY FOR DIFFUSE CORONARY ARTERY DISEASE: TEN YEARS CLINICAL AND ANGIOGRAPHIC RESULTS}

\author{
C.H. David, E. Bezon, B. Provost, A.A. Khalifa, J.N. Choplain \\ Brest University Medical School Hospital, Department of Cardiovascular and Thoracic Surgery, \\ Brest, France
}

\begin{abstract}
Aims: Diffuse coronary artery disease makes cardiac surgeons hesitant whether coronary artery bypass graft is realizable or not. The coronary artery reconstruction using the internal thoracic artery allows bypassing coronary arteries with diffuse atheromatous plaques without systematic endarterectomy. The aim of the study was to evaluate the 10 years results of the coronary artery reconstruction of the left anterior descending artery.
\end{abstract}

Method: All the 118 patients undergoing at least a coronary artery reconstruction of the left anterior descending artery between 1999 and 2000 were included in the study. The mean age was $57 \pm 8$ years. Bilateral internal thoracic arteries were used in 92 patients (78\%), 269 coronary artery bypass grafts were performed. The mean length of the coronary artery reconstruction of the LAD was $4.3 \pm 2 \mathrm{~cm}(1.5-12 \mathrm{~cm})$. An endarterectomy was associated in 16 patients $(14 \%)$. Follow-up data were compiled between August 2009 and January 2010.

Results: In-hospital mortality was $0.8 \%$ (1 patient). Mean follow-up was $118 \pm 11$ months. At the time of the study, 101 patients were alive (86\%), 81 patients were free of major cardiac events $(80 \%)$. The patency of the coronary artery reconstruction was evaluated by either coronary angiography (64 patients) or coronary computed tomography (25 patients). The 10 years patency of the coronary artery reconstruction of the left anterior descending artery is $94 \%$ (84/89).

Conclusion: The coronary artery reconstruction using the internal thoracic, give excellent long term outcome to the diffuse left anterior descending artery diseased patients.

No conflict of interest 


\section{CARDIAC SURGERY: CURRENT ISSUES}

\section{HOW TO REACH THE TARGET FLOW IN MINIMAL INVASIVE PEDIATRIC HEART PERFUSION}

S. Abdel-Sayed $^{1}$, K.L. von Segesser ${ }^{2}$

${ }^{1}$ Cardiovascular research, Lausanne, Switzerland

${ }^{2}$ Cardiovascular research unit, Surgery, Lausanne, Switzerland

Aims: The aim of this study was to assess whether smaller diameters of the virtually wall-less venous cannulas designed for use with augmented and venous drainage can provide improvements of drainage with in smaller paediatric access orifices.

Method: Flow rate $(Q)$ and negative pressure $(P)$ were measured for the 24F wall-less (Smartcanula) restricted to $17 \mathrm{~F}$, and the control (Biomedicus cannula) 17F, using centrifugal pump and an experimental bench set-up, with after load of $60 \mathrm{mmHg}$.

Results: 144 pair measurements were recorded. At 1500, 2000, 2500, and 3000 RPM pump speed, the flow for the $17 \mathrm{~F}$ access were $2.62 \pm 0.02,4.66 \pm 0.03,6.47 \pm 0.08$, and $8.21 \pm 0.09$ $\mathrm{I} / \mathrm{min}$ for the wall-less cannula, versus $1.56 \pm 0.03^{*}, 2.77 \pm 0.02^{*}, 3.82 \pm 0.05^{*}$, and $4.77 \pm 0.05^{*}$ : $\mathrm{p}<0.05^{\star} \mathrm{I} / \mathrm{min}$ for the control cannula. The pressure were $-28.58 \pm 0.14,-70.62 \pm 0.41,-124.49 \pm$ 0.43 , and $-191.20 \pm 1.11 \mathrm{mmHg}$ for wall-less cannula, versus $-37.38 \pm 0.66^{*},-95.54 \pm 0.61^{*}$, $170.25 \pm 1.43^{*}$, and $-257.55 \pm 1.05^{\star} \mathrm{mmHg}^{*}$ for the control cannula.: $\mathrm{p}<0.05^{*}$.

Conclusion: The walls-less cannula provides a promising solution for improved venous drainage for scenarios, with $17 \mathrm{~F}$ access. One single cannula diameter for various access vessel diameters simplifies minimal invasive cannulation.

No conflict of interest 
211

CARDIAC SURGERY: CURRENT ISSUES

\section{SURGICAL REVASCULARIZATION OF CHRONICALLY OCCLUDED RIGHT CORONARY ARTERY}

A. Borowski, E. Godehardt, H. Dalyanoglu

University of Düsseldorf, Clinic for Cardiovascular Surgery, Düsseldorf, Germany

Aims: Chronic totally occluded right coronary artery (CTO-RCA) often poses a problem in decision making for/against bypass grafting due to the lack of standardized indication criteria.

Method: A retrospective study was conducted with patients who underwent coronary artery bypass grafting including single graft to the RCA. The distal run-off of the bypassed RCA as a predictor of long term graft patency was measured intraoperatively using ultrasonic transit-time method, and analysed in regard to diameter, donor sources, Rentrop class, collateral pathway, regional contractility, and comorbidity.

Results: Sixty nine patients were enrolled in the study. Significantly lower flow $(p<0.0001)$ and diameter values $(p<0.05)$ of the recipient artery were observed in patients with regional hypo/akinesia and Q-infarction of the posterior wall. Diabetes mellitus seems to compromise the flow capacity of the CTO-RCA-territory.

Conclusion: In decision making for/against bypass grafting of the CTO-RCA, preoperative evaluation of the myocardial viability seems to be indispensable in order to avoid redundant or even harmful revascularization.

No conflict of interest 
212

CARDIAC SURGERY: CURRENT ISSUES

\title{
WHO MIGHT BENEFIT FROM EARLY ASPIRIN AFTER CORONARY ARTERY SURGERY?
}

\author{
P. Gukop, N. Gutman, R. Bilkhu, A. Shety, G.T. Karapanagiotidis, A. Momin \\ St George's Hospital NHS Trust, Department of Cardio-thoracic Surgery, London, \\ United Kingdom
}

\begin{abstract}
Aims: Early postoperative aspirin (300mg) administered within 6 hours following CABG has been shown to be optimal for prevention of saphenous vein graft (SVG) occlusion. Early aspirin has shown significant benefit in reducing SVG occlusion, MI, stroke, renal failure and bowel infarction, and subsequent mortality. The efficacy of early post-operative aspirin on vein graft patency diminishes the later it is administered. It has optimal benefit at 6 hours, significantly reduced benefit at 24 hours and no benefit after 48hours post CABG. However the maximum benefit has been demonstrated when administered at one hour post CABG.
\end{abstract}

Method: ACC/AHA, EACTS and ACCP have issued guidelines recommending administration of early aspirin or an alternative (clopidogrel, ticlopidine and indobufen) at 6hours or soon after bleeding has settled as the standard of care for optimisation of SVG patency. The ACCP guideline has also suggested that optimal prevention of cardiovascular complication should merit higher value than the small risk of post-operative bleeding.

Several randomised controlled studies (level 1 evidence) including a meta-analysis have shown that administration of early aspirin following CABG is not associated with increased blood loss or transfusion requirements.

Post-operative bleeding has been identified as a significant reason for non-administration of early aspirin in a prospective study performed at our own institution.

Results: It is essential to define/quantify the postoperative blood loss that precludes administration of early aspirin.

Conclusion: This will enhance prompt administration in some cases and guide judgement especially in patients with high risk factors for vein graft thrombosis.Administration at 6 hours is the optimal time to give aspirin as long as bleeding has settled. Individualising the administration of early aspirin based on clinical judgement on case to case basis would maximise its benefit. We thus advocate a bespoke method of early aspirin administration post CABG.

No conflict of interest 
213

CARDIAC SURGERY: CURRENT ISSUES

DO WE BRING DOWN LONG-TERM SURVIVAL IN PATIENTS WITH SEVERE ISCHEMIC MITRAL REGURGITATION WHEN REPLACE MITRAL VALVE: PROPENSITY-MATCHED ANALYSIS?

\author{
V. Shumavets ${ }^{1}$, A. Shket ${ }^{1}$, G. Irina' ${ }^{2}$, S. Kurganovich ${ }^{2}$, E. Lysjonok ${ }^{1}$, Y. Ostrovsky ${ }^{1}$ \\ ${ }^{1}$ Belarus Cardiology Centre, Cardiac surgery, Minsk, Belarus \\ ${ }^{2}$ Belarus Cardiology Centre, Echocardiography, Minsk, Belarus
}

Aims: Our study focused on patients with severe compromised ischemic left ventricle and functional IMR to understand how mitral valve (MV) replacement versus repair affects survival.

Method: From 1068 operated patients (mean age 57,9 $\pm 8,3$ years) CABG were combined in 989 patients with MV repair and in 79 patients - MV replacement. Groups were matched by propensity score. Survival (with mean follow-up 7,34 $\pm 1,8$ years) and NYHA class were compared. Uni- and multivariate analyses were performed.

Results: Before matching 10-year survival was significantly worse in replacement group (HR$2,14 ; 95 \% \mathrm{Cl} 1,43-3,21 ; \mathrm{p}=0,001)$. After propensity matching $1: 1$ we've received cohort of 138 patients with severe compromised LV (EDD 71,1 \pm 7,9 mm, iEDV $126 \pm 33,9 \mathrm{ml} / \mathrm{m2}$, EF 31,5 \pm $5,7 \%$ ) and severe MR (ERO PISA 0,43 $\pm 0,18 \mathrm{~mm} 2$ ). The fact of MV replacement versus repair did not impair long-term survival (HR- 1,35; $95 \% \mathrm{Cl} 0,82-2,29 ; \mathrm{p}=0,26)$. NYHA class in followup was comparable between matched groups (with mean 2,38 $\pm 0,68$ after MV replacement vs. $2,25 \pm 0,92$ for MV repair, $\chi 2-5,29, p=0,49$ ). In multivariate Cox regression LV ESD (HR$1,038,95 \% \mathrm{Cl} 1,004-1,072, p=0,013)$ and advanced NYHA (HR- 2,55, 95\% Cl 1,57-6,1, p = 0,037 ) were found as independent risk-factors for an increased long-term mortality.

Conclusion: The MV chordal-sparing replacement versus repair did not take down survival in patients with severe damaged ischemic LV. Survival mostly depends on factors related to the patient's condition at the time of surgery.

No conflict of interest 


\title{
371
}

\section{VENTRICULAR FUNCTION, HEART FAILURE}

\section{AGING AND HEART FAILURE 2015: ROLE OF EXTRACELLULAR MATRIX}

\author{
B.l. Jugdutt \\ University of Alberta, Edmonton, Alberta, Canada
}

The aging population with heart failure (HF) worldwide has been increasing steadily. In elderly patients aged $\geq 65$ years, the two main comorbidities leading to HF are hypertension (HTN) and myocardial infarction (MI). Aging results in progressive cardiovascular changes and the aging phenotype can impact negatively on disease expression and response to therapy. Aging-related changes per se contribute to adverse cardiac remodeling and HF with preserved ejection fraction (HFpEF). HTN also leads to HFpEF while MI leads to HF with reduced EF (HFrEF). Aging and concomitant HTN or MI aggravate HFpEF or HFrEF phenotypes and accelerate the march to HF. The extracellular matrix (ECM) plays a key role in maintaining cardiac shape and function and is a key factor in the exacerbation of and march to HF in HTN and MI. Dysregulation of ECM homeostasis and metabolism and disruption of the ECM network exacerbate adverse cardiac remodeling, shape deformation and dysfunction that spur on the march to HF, disability and death. In older patients, aging-related cardiac remodeling with superimposed progressive left ventricular remodeling leading to HFpEF or HFrEF is a persistent problem that has important therapeutic implications. Studies suggest that in the elderly, novel pathways can be targeted for optimizing therapy in HFrEF post-MI and HFpEF post-HTN. Therapeutic strategies that include targeting of adverse cardiac ECM remodeling could prevent, limit and reverse progression of HF in aging patients.

Key words: aging, extracellular matrix, hypertension, myocardial infarction, remodeling, heart failure

No conflict of interest 
VENTRICULAR FUNCTION, HEART FAILURE

\title{
TREATMENT OF A REPRODUCIBLE RAT MODEL OF MYOCARDIAL INFARCTION WITH DIFFERENTIATED HBMSCS ON A NANOSCAFFOLD SYSTEM
}

\author{
E. Marks, K. Arun \\ University of Delaware, Medical Laboratory Sciences, Newark, USA
}

Aims: It is a long time struggle to create invasive animal models for myocardial infarction (MI) that only mimic single pathologies instead of the wide range seen clinically. We have therefore developed a reproducible model of MI which is relevant to MI pathologies in a dose dependent manner and used nanofiber based scaffolds with human bone marrow derived mesenchymal stem cells(hBMSc) to repair the damaged heart.

Method: We have developed a reproducible model of MI using single subcutaneous injections of isoproterenol $(0-150 \mathrm{mg} / \mathrm{kg})$ that result in clinically relevant MI pathologies in a dose dependent manner. An image-guided laparoscopic injection of nanofiber scaffolding system housing human bone marrow stem cells (hBMSCs) was then used to treat the varied MI pathologies.

Results: Histological analysis of cardiomyocyte hypertrophy and apoptosis, cardiac metrics (LV diameter(s), EF, CO, etc.), and creatinine kinase biomarkers of the models all align with published clinical values. In vitro interaction of hBMSCs with thymosin $\beta 4$ and IWP-2 resulted in cardiac lineage differentiation based on desmin, $\alpha$-actinin, troponin I, ANP, and tropomyosin biomarker staining. Injections of the nanoscaffold system to the isoproterenol rat model(s) increased EF and prevented mortality in all rat models, as determined through H\&E staining and decreases in creatinine kinase.

Conclusion: Injections of the nanoscaffold system to the isoproterenol rat model(s) increased EF and prevented mortality in all rat models, and prevented extended tissue damage as determined through H\&E staining and decreases in creatinine kinase. We believe a tailored nanoscaffolding system will increase treatment options for moderate to severe MI.

No conflict of interest 


\title{
219
}

\section{VENTRICULAR FUNCTION, HEART FAILURE}

\section{OVEREXPRESSION OF HUMAN A2A RECEPTORS PROTECT THE HEART AGAINST ISCHEMIA}

\author{
J. Neumann ${ }^{1}$, U. Gergs ${ }^{1}$, F.U. Müller ${ }^{2}$, J. Eskandar', K. Drzewiecki², W. Schmitz², P. Boknik ${ }^{2}$ \\ ${ }^{1}$ Martin-Luther-University Halle-Wittenberg, Institute of Pharmacology and Toxicology- \\ Medical Faculty, Halle Saale, Germany \\ ${ }^{2}$ University Hospital, Institute of Pharmacology and Toxicology, Münster, Germany
}

Adenosine is formed during ischemia in the heart of many species including humans. There is evidence that it can protect the myocardial integrity and function during reperfusion. However, the underlying mechanisms are poorly understood. Adenosine can act in part, via G-protein coupled receptors usually classified as A1-, A2a-, A2b- and A3-receptor. Their expression in cardiac regions, cellular localization and signal transductions mechanisms are quite divergent. Whereas contractile effects of adenosine via A1 receptors are well studied, much less is known about A2a receptors. Typical $\mathrm{A} 2 \mathrm{a}$ receptor agonists are inactive isolated atrial or ventricular preparations of wild type mice. Hence, we have generated mice which overexpress human A2a receptors in the mouse heart, using the cardiomyocytes specific alpha myosin heavy chain promoter (TG). In TG typical A2A agonists like CGS21680 (0.1-10 $\mu \mathrm{M})$ exerted positive inotropic effects in an A2a receptor specific manner (being blocked by an A2a receptor antagonist, ZM 2141385, $1 \mu \mathrm{M}$ ). In isolated perfused hearts subjected to global ischemia (20,40,60 min) and reperfusion (40 min), we noted that TG hearts regained mechanical function left ventricular pressure, or first derivative of pressure) faster and to a higher extent than WT. Moreover, these beneficial effects in TG were abrogated when TG were pretreated with the A2a blocker SCH442416 $(0.1 \mu \mathrm{M})$. These data suggest that a protective effect of adenosine in the heart can occur via A2a receptors. It is tempting to speculate that similar protective effects might be elicited by A2a receptor agonistic drugs in patients.

No conflict of interest 


\title{
BASIC RESEARCH, MOLECULAR BIOLOGY
}

\section{LEUKOCYTE TELOMERE LENGTH IS NOT A PREDICTOR OF THE SURVIVAL LENGTH IN PATIENTS AFTER HEART TRANSPLANTATION - 20 YEARS FOLLOW UP}

\author{
D. Dlouha1, V. Vancura², J. Vymetalova², J. Hubacek ${ }^{1}$ V. Lanska ${ }^{3}$, I. Malek ${ }^{2}$ \\ ${ }^{1}$ Institute for Clinical and Experimental Medicine, Center for Experimental Medicine, Prague, \\ Czech Republic \\ ${ }^{2}$ Institute for Clinical and Experimental Medicine, Department of Cardiology, Prague, \\ Czech Republic \\ ${ }^{3}$ Institute for Clinical and Experimental Medicine, Statistical Unit, Prague, Czech Republic
}

Aims: Leukocyte telomere length $(L T L)$ is getting shorter progressively with age, and is reported to be inversely associated with aging-related diseases including cardiovascular disease. If LTL could reflect survival length of patients after heart allograft transplantation was hypothesis of our study.

Method: We have measured LTL with a quantitative PCR-based method in DNA extracted from 132 blood samples of heart recipients ( 111 males, 21 females, age $44.3 \pm 10.4$, who survived at least 1 month to 30 years after transplantation) with indication of dilated cardiomyopathy or ischaemic heart disease for cardiac transplant followed for up to 20 years. Leukocyte DNA was extracted using the salting-out protocol and LTL was measured with a quantitative PCR-based method.

Results: LTL was not significantly correlated with overall survival after heart transplantation $(P=$ $0.55)$. LTL in patients with non-cardiac cause of death was significantly shorter than in those with cardiac cause $(P<0.01, P<0.05$ after adjustment for age). In addition, we found different trend in LTL dependence on the age in subjects with cardiac and non-cardiac cause of death $(\mathrm{P}<$ 0.05).

Conclusion: LTL was not associated with general survival length in patients after heart transplantation, but shorted LTL was a marker of non-cardiac cause of death. The absence of significant association observed here should be interpreted with caution and could be caused by the small sample size and low study power.

Supported by project No. NT 14023-3/2013 (IGA, MH, CR).

No conflict of interest 


\title{
BASIC RESEARCH, MOLECULAR BIOLOGY
}

\section{THE MITOCHONDRIAL RESERVE RESPIRATORY CAPACITY IS REGULATED BY AMPK, PYRUVATE DEHYDROGENASE KINASE, AND SIRT3, AND ENHANCES RESISTANCE TO CELL DEATH}

\author{
J. Pfleger, M. He, M. Abdellatif \\ Rutgers - New Jersey Medical School, Cellular Biology and Molecular Medicine, Newark, USA
}

\begin{abstract}
Aims: One of the main causes of myocyte damage during cardiac failure is attributable to mitochondrial dysfunction and, therefore, inability to meet energy demand and contractile function. We hypothesized that mitochondrial reserve respiratory capacity (RRC) is a critical bioenergetic component that is utilized during increased energy demand, enhancing viability. Our goal was to identify the elements that regulate and contribute to RRC and its involvement in cell survival.
\end{abstract}

Method: The Seahorse XF-24 Analyzer was used to measure oxygen consumption rates (OCR) and calculate RRC in neonatal rat cardiac myocytes (NRCM).

Results: We found that RRC is dependent on metabolic substrate availability and cell type. In $\mathrm{NRCM}$, developing RRC required fatty acids in addition to glucose. Accordingly, inhibition of either glucose or fatty acid oxidation abrogated RRC, having little impact on basal OCR. Conversely, RRC was enhanced through increasing glucose oxidation via inhibiting pyruvate dehydrogenase kinases with dichloroacetate, or through increasing fatty acid oxidation via activation of AMP-activated kinase (AMPK). An electron flow activity assay revealed that increases in RRC correlated with increases in complex II (cll) activity. Inhibiting or disassembling cll abolished RRC, confirming it as the source of RRC. Moreover, RRC required Sirt3. We show that enhancing RRC via fatty acid oxidation with 5-Aminoimidazole-4-carboxamide 1- $\beta$ Dribofuranoside in NRCM results in a burst of cll-dependent oxidative phosphorylation accompanied by reduced superoxide production enhanced cell survival post-energy deprivation.

Conclusion: We show that metabolic sensors increase RRC via activating cll in a Sirt3dependent manner, and that this mechanism can be exploited for increasing cell survival after hypoxia.

No conflict of interest 


\title{
BASIC RESEARCH, MOLECULAR BIOLOGY
}

\section{LOSS OF CAVEOLIN-1 PROTECTS AGAINST PCB-INDUCED VASCULAR DYSFUNCTION BY UPREGULATING NRF2 AND THE ANTIOXIDANT RESPONSE}

\author{
B. Hennig \\ University of Kentucky, Superfund Research Center, Lexington, USA
}

\begin{abstract}
Aims: Environmental persistent organic pollutants such as polychlorinated biphenyls (PCBs) have been implicated in multiple inflammatory disorders including cardiovascular disease. Information regarding mechanisms of toxicity and cross-talk between relevant cell signaling pathways is lacking.
\end{abstract}

Method: Numerous inflammatory genes are anchored within membrane domains called caveolae, and we examined the cross-talk between caveolae and nuclear factor (erythroidderived 2)-like 2 (Nrf2) pathways on the regulation of PCB-induced inflammation.

Results: Caveolin-1 silencing in vascular endothelial cells resulted in a decreased PCB-induced inflammatory response, and also in increased levels of Nrf2-ARE transcriptional binding, resulting in higher mRNA levels of the antioxidant genes in both vehicle and PCB-treated systems. Along with this upregulated antioxidant response, caveolin-1 siRNA treated cells exhibited decreased mRNA and protein levels of Nrf2 inhibitory proteins Keap1 and Fyn kinase, especially in PCBtreated cells. Further, endothelial cells from wildtype and caveolin-1-/- mice were isolated and treated with PCB to better elucidate the role of functional caveolae in PCB-induced endothelial inflammation. Caveolin-1-/- endothelial cells were protected from PCB-induced cellular dysfunction as evidenced by decreased vascular cell adhesion molecule (VCAM-1) protein induction. Compared to wildtype cells, caveolin-1-/- endothelial cells also allowed for a more effective antioxidant response, as observed by higher levels of the antioxidant genes.

Conclusion: These results suggest that functional caveolae are required for induction of inflammatory genes. Our data also demonstrate novel cross-talk mechanisms between caveolae and Nrf2 and implicate the reduction of caveolin-1 as a protective mechanism for PCB-induced cellular dysfunction and inflammation.

No conflict of interest 


\title{
BASIC RESEARCH, MOLECULAR BIOLOGY
}

\section{QUERCETIN IMPROVES POSTISCHEMIC RECOVERY OF HEART FUNCTION IN DOXORUBICIN-TREATED RATS AND PREVENTS DOXORUBICIN-INDUCED APOPTOSIS IN RAT HEARTS}

\author{
M. Bartekova ${ }^{1}$, M. Fogarassyova ${ }^{2}$, M. Barancik ${ }^{2}$ \\ ${ }^{1}$ Institute for Heart Research, Cardiovascular Physiology and Pathophysiology, Bratislava, \\ Slovakia \\ 2Institute for Heart Research, Protein Chemistry, Bratislava, Slovakia
}

\begin{abstract}
Aims: The aim of the present study was to explore effects of prolonged administration of quercetin on changes induced by repeated application of anthracycline antibiotic doxorubicin in rat hearts. We focused on the ischemic tolerance and apoptosis induction in rat hearts, as well as on determining possible mechanisms underlying effects of quercetin.
\end{abstract}

Method: Rats were treated with doxorubicin in cumulative doses of $15 \mathrm{mg} / \mathrm{kg}$ for 3 weeks. Doxorubicin was applied by seven intraperitoneal injections, every 3rd day. Quercetin was served on a piece of bisquit in the dose of $20 \mathrm{mg} / \mathrm{kg} /$ day. The administration of quercetin started on the same day as the doxorubicin administration and continued for further 3 weeks after the completion of the doxorubicin treatment (total period of 6 weeks).

Results: Our results showed that rat hearts exposed to effects of quercetin were more resistant to ischemia/reperfusion injury in both control and doxorubicin-treated rats. Effects of quercetin on modulation of ischemic tolerance were linked to Akt kinase activation followed by GSK-3 $\beta$ downregulation and elevation of $\beta$-catenin. Moreover, we showed that quercetin reversed effect of doxorubicin on apoptosis induction.

Conclusion: In conclusion, our results demonstrate that prolonged treatment with quercetin prevented negative chronic effects of doxorubicin on apoptosis induction, and positively influenced myocardial responses to acute ischemic stress. Additionally, our data suggest important role of Akt/GSK-3 $\beta / \beta$-catenin pathway in quercetin action in the heart. These findings bring new insights into mechanisms of quercetin action in the hearts exposed to chronic effects of doxorubicin.

Supported by VEGA SR 2/0140/12, 2/0108/15 and APVV-0348-12

No conflict of interest 


\title{
226111 - BASIC RESEARCH, MOLECULAR BIOLOGY \\ ASSOCIATION OF POLYMORPHISMS IN TUMOR NECROSIS FACTOR ALPHA GENE WITH CORONARY POST-ANGIOPLASTY RESTENOSIS
}

\author{
$\underline{\text { F. Lanas }}^{1}$, B. Bobadilla ${ }^{2}$, J. Ruedlinger ${ }^{3}$, N. Saavedra ${ }^{3}$, L. Perez ${ }^{4}$, M. Potthoff ${ }^{5}$, C. Olivares ${ }^{6}$, \\ L. Salazar ${ }^{3}$ \\ 1 Universidad de La Frontera, Internal Medicine, Temuco, Chile \\ ${ }^{2}$ Hospital Hernan Henriquez, Cardiology, Temuco, Chile \\ ${ }^{3}$ Universidad de La Frontera, Basic Sciences, Temuco, Chile \\ ${ }^{4}$ Universidad de Concepcion, Internal Medicine, Concepcion, Chile \\ ${ }^{5}$ Universidad de La Frontera, Internist, Temuco, Chile \\ ${ }^{6}$ Universidad de La Frontera, Cardiology, Temuco, Chile
}

\begin{abstract}
Aims: Increased pre-procedural tumor necrosis factor alpha (TNF-alpha) levels during percutaneous transluminal coronary angioplasty (PTCA) are predictors of restenosis. However, studies about TNF-alpha polymorphisms and angiographic restenosis have provided discordant results. The aim of this work was to assess the association between TNF-alpha polymorphisms with PTCA restenosis
\end{abstract}

Method: 194 patients who underwent a coronary angiography and had a history of previous PTCA, at least 6 months before, were enrolled. Cases had stenosis diameter $>50 \%$ of the index lesion at follow-up angiography and controls stenosis $\leq 50 \%$. Demographic and clinical variables were recorded, and polymorphisms rs361525 (G>A) and rs1799964 $(T>C)$ of TNF-alpha gene were determined by real time PCR

Results: 88 participants were identified as cases and 106 as controls. No differences were observed in age, gender and diabetes mellitus prevalence: $35.4 \%$ vs. $24.0 \%$; OR $1.7(95 \% \mathrm{Cl}$ 0.7-4.0) $p=0.20$, No difference was observed in the frequency of genotypes for the rs 361525 polymorphism: $\mathrm{G} / \mathrm{G}$ and $\mathrm{A} / \mathrm{G}$ frequency in cases $87.8 \%$ and $12.2 \% \%$ vs $84.3 \%$ and $15.7 \%$, respectively, in controls, OR $1.34(95 \% \mathrm{Cl} 0.57-3.13) \mathrm{p}=0.25$. Similarly, no difference was observed in the frequency of genotypes for the rs 1799964 polymorphism in cases and controls: CC: 2.5 vs $2.0 \% \mathrm{C} / \mathrm{T}: 33.3$ vs $35.3 \%$ and $\mathrm{T} / \mathrm{T}: 64.2$ vs $62.7 \%$, respectively. Allele $\mathrm{C}$ frequency $37.2 \%$ in cases vs. $35.8 \% \%$ in controls; OR $1.29(95 \% \mathrm{Cl}=0.72-2.3), \mathrm{p}=0.39$

Conclusion: No association was observed between polymorphisms rs361525 (G>A) and rs1799964 (T>C) in the TNF-alpha gene and coronary restenosis after angioplasty

Financial support: FONDECYT- Chile ( $\left.\mathrm{N}^{\circ} 1141292\right)$

No conflict of interest 


\title{
BASIC RESEARCH, MOLECULAR BIOLOGY
}

\section{MATRIX METALLOPROTEINASES ARE INVOLVED IN CHRONIC EFFECTS OF DOXORUBICIN ASSOCIATED WITH BLOOD PRESSURE INCREASE IN RATS AND QUERCETIN POSSESSES BENEFICIAL EFFECTS AGAINST DOXORUBICIN-INDUCED CHANGES}

\author{
M. Barancik ${ }^{1}$, I. Dovinova ${ }^{2}$, P. Simoncikova ${ }^{1}$, M. Fogarassyova ${ }^{1}$, M. Bartekova ${ }^{3}$ \\ ${ }^{1}$ Institute for Heart Research Slovak Academy of Sciences, Laboratory of Protein Chemistry, \\ Bratislava, Slovakia \\ ${ }^{2}$ Institute of Normal and Pathological Physiology- Slovak Academy of Sciences, \\ Laboratory of Neurohumoral Regulation of Hemodynamics, Bratislava, Slovakia \\ 3 Institute for Heart Research Slovak Academy of Sciences, \\ Department of Cardiovascular Physiology and Pathophysiology, Bratislava, Slovakia
} Aims: To investigate the role of matrix metalloproteinases (MMPs) in responses of rats to
prolonged doxorubicin (DOX) treatment. Effects of potential protective substance, flavonoid
quercetin (QCT), on DOX-induced effects were also determined.

Method: In this study, male Wistar rats were used. DOX was administered in cumulative dose 15 mg. $\mathrm{kg}^{-1}$. QCT was applied during and three weeks after DOX treatment $(20 \mathrm{mg} / \mathrm{kg} / \mathrm{day})$. Samples were collected four and eight weeks after end of DOX application. Systolic blood pressure (SBP) was measured by tail cuff plethysmography. Protein levels were determined by immunoblot assay and MMP activities were measured by gelatin zymography. Superoxide dismutase (SOD) activities were analyzed with SOD assay kit. Structural alterations of heart were characterized by transmission electron microscopy.

Results: DOX induced heterogeneous subcellular alterations of cardiomyocytes and structural disorganizations of cardiac extracellular space. SBP blood pressure was higher in DOX-treated rats eight weeks after treatment. Effects of DOX were linked to stimulation of plasma MMP activities. In the left ventricle, application of DOX led to MMP-2 activation eight weeks after end of DOX-treatment. Changes in tissue MMP-2 were connected with SOD inhibition and iNOS induction. QCT prevented DOX-induced effects on MMP-2 and influenced also other negative chronic effects of DOX (SBP increase; deleterious effects on ultrastructure; SOD inhibition).

Conclusion: Our results show that MMPs are involved in chronic cardiotoxic effects of DOX. The study also shows that prolonged treatment with QCT possesses beneficial effects against DOXinduced changes in rat hearts as evident from lowered SBP, attenuated tissue MMP-2 activation, and decreased cellular damage.

No conflict of interest 


\title{
INTERVENTIONAL AND STENTING
}

\section{EFFECTS OF ADMINISTRATION OF DUAL ANTIPLATELET THERAPY (DAPT) FOR THREE MONTHS VS. TWELVE MONTHS ON CLINICAL OUTCOMES OF PATIENTS AFTER DRUG- ELUTING STENT IMPLANTATION: A METAANALYSIS}

\author{
J.R. Santos, N.L. Bagsit, L. Valera, M.B. De Guzman \\ University of Santo Tomas Hospital, Heart Station- Second Floor- Private Division, Manila, \\ Philippines
}

Aims: To analyze the effect of dual antiplatelet therapy (DAPT) for 3 months versus 12 months on clinical outcomes of patients after DES implantation.

\begin{abstract}
Method:
DESIGN: Systematic search was done and three trials were included. OPTIMIZE (Three vs Twelve Months of Dual Antiplatelet Therapy After Zotarolimus-Eluting Stents and RESET (Real Safety and Efficacy of Three-month Dual Antiplatelet Therapy following Endeavor Zotarolimuseluting Stent Implantation) are multicenter, open label randomized trials, while DATE (Duration of Dual Antiplatelet Therapy After Implantation of Endeavor Stent) trial is a prospective multicenter cohort study.

PARTICIPANTS: This meta-analysis included 6048 subjects, 3283 were given DAPT for 3 months, while 2765 were given clopidogrel for 12 months.

OUTCOMES: Effect on all-cause mortality, occurrence of myocardial infarction, rate of stent thrombosis and bleeding were determined.

Results: No significant differences in all-cause mortality ( $p$ value 0.19 ), occurrence of myocardial infarction ( $p$ value 0.68 ), and rate of stent thrombosis ( $p$ value 0.89 ) were noted among patients given DAPT for 3 months versus 12 months. However, bleeding was significantly reduced ( $p$ value 0.008), favoring DAPT for 3 months

Conclusion: Effect on all-cause mortality, myocardial infarction, and stent thrombosis were similar in 3 months and 1 year of DAPT. However, 3 months DAPT resulted in lesser bleeding events.
\end{abstract}

No conflict of interest 


\title{
INTERVENTIONAL AND STENTING
}

\section{TEN-YEAR CLINICAL OUTCOMES OF SIROLIMUS-ELUTING STENT VERSUS BARE-METAL STENT; A MATCHED ANALYSIS FROM A REAL WORLD, SINGLE CENTER REGISTRY}

\author{
J.S. Park, J.H. Nam, C.H. Lee, J.W. Son, U. Kim, D.G. Shin, Y.J. Kim \\ Yeungnam University Medical Center, Internal Medicine, Daegu, Korea
}

\begin{abstract}
Aims: The aim of this study is to compare clinical outcomes for ten years, between sirolimuseluting stent (SES) and bare metal stent (BMS).

Method: During the BMS and drug-eluting stent (DES) transition period (from April 2002 to April 2004), 434 consecutive patients with 482 lesions underwent percutaneous coronary intervention, using BMS or SES. Using propensity score matching, 187 patients with BMS and 166 patients with SES were selected. 10 year clinical outcomes of major adverse cardiac events (MACE) such as myocardial infarction (MI), ischemia-driven target vessel revascularization (TVR) and all-cause of death, and angiographic definite stent thrombosis (ST) were compared.
\end{abstract}

Results: At one-year follow up, patients with SES showed significantly lower MACE $(11.2 \%$ in BMS vs $4.8 \%$ in SES, $P=0.029$ ). However, cumulative MACE for 10 year was not significantly different between two groups (40.6\% in BMS vs $40.4 \%$ in SES, $P=0.957$ ). There were no significant differences in MI, ischemia-driven TVR, all-cause of death and ST for 10 year. The TVR was gradually increased from 1 to 10 year in SES, on the contrary to that of BMS.

Conclusion: In conclusion, although SES showed better clinical outcomes in the early period after implantation, it did not show significant benefits in the long-term follow up, compared with that of BMS.

No conflict of interest 


\title{
INTERVENTIONAL AND STENTING
}

\section{TRANSCATHETER AORTIC VALVE IMPLANTATION IN PATIENTS WITH SEVERE AORTIC STENOSIS AND LEFT VENTRICULAR DYSFUNCTION: IMMEDIATE RESULTS AND MEDIUM TERM FOLLOW UP}

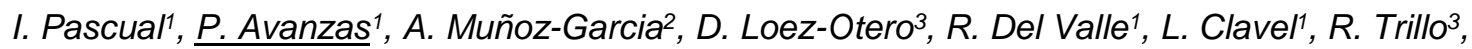 \\ J.M. Hernandez ${ }^{2}$, C. Morís ${ }^{1}$ \\ ${ }^{1}$ Hospital Central de Asturias, Cardiology, Oviedo, Spain \\ ${ }^{2}$ Hospital Universitario Virgen de la Victoria., Cardiology, Malaga, Spain \\ ${ }^{3}$ Complejo Hospitalario de Santiago., Cardiology, Santiago de Compostela, Spain
}

Aims: The main objective of this study was to evaluate immediate results and medium term follow-up of transcatheter aortic valve implantation (TAVI) in patients with severe aortic stenosis (AS) and left ventricular dysfunction (LVD). We also compare data and mortality rates between patients with severe left ventricular dysfunction (SLVD) and patients with might to moderate left ventricular dysfunction.

Method: Multicenter, observational and prospective study carried out in 3 hospitals. We included consecutive patients with severe AS and LVD treated with TAVI, The primary endpoint was to evaluate death from any cause at 2 years.

Results: One hundred consecutive patients with severe AS and LVD were included. 23 patients $(23 \%)$ had severe LVD (SLVD) $(<30 \%)$ and 70 patients had might to moderate LVD (MLVD) (30$50 \%)$. SLVD group had higher mean EuroSCORE than MLVD $(32,2 \pm 18,2$ vs 23,2 $\pm 15,9$; $p=0,025)$ and lower rates of procedural success $(98,7$ vs $87 \%, p=0,032)$. After 24 months of follow up there were no significant differences between mortality rates between two groups [7 $(30,4 \%)$ vs $13(16,9 \%) ; p=0,23]$. Figure shows survival curves. Cox proportional hazard regression showed no significant independent predictors of mortality. 


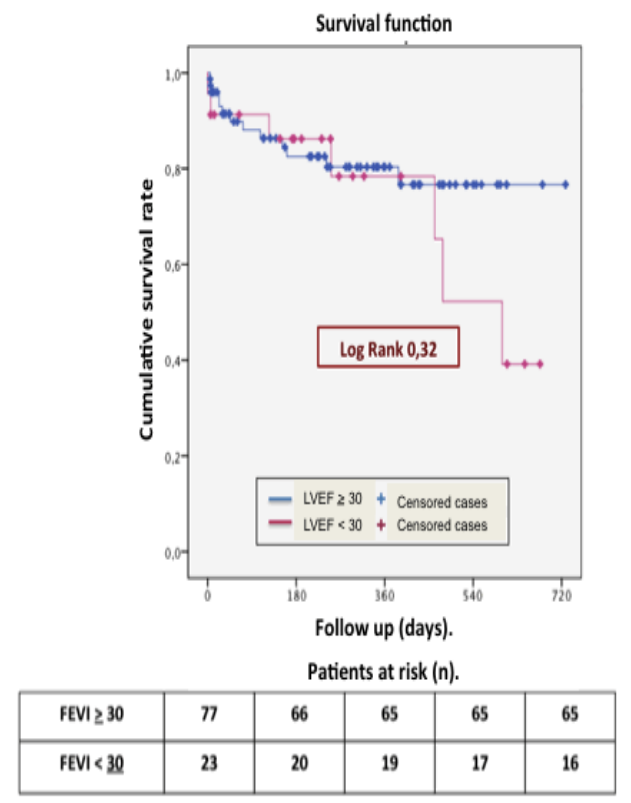

Conclusion: TAVI is safe and effective in a selected population of patients with severe aortic stenosis and left ventricular dysfunction. Severe left ventricular dysfunction wasn't an independent predictor of two years mortality. Nowadays, patients with severe left ventricular dysfunction had lower rates of procedural success than patients with might to moderate left ventricular dysfunction.

No conflict of interest 


\title{
233
}

\section{INTERVENTIONAL AND STENTING}

\section{NOS3 GENE POLYMORPHISMS IN CHILEAN PATIENTS WITH POST-ANGIOPLASTY RESTENOSIS}

\author{
L.A. Salazar ${ }^{1}$, J. Ruedlinger ${ }^{1}$, N. Saavedra ${ }^{1}$, B. Bobadilla², L. Pérez³, F. Lanas ${ }^{1}$ \\ ${ }_{1}^{1}$ Universidad de La Frontera, Center of Molecular Biology \& Pharmacogenetics, Temuco, Chile \\ ${ }^{2}$ Universidad de La Frontera, Faculty of Medicine, Temuco, Chile \\ ${ }^{3}$ Universidad de Concepción, Faculty of Medicine, Concepción, Chile
}

\begin{abstract}
Aims: To date, several reports indicate that a decrease in the expression of nitric oxide synthase (NOS) is related to the risk of coronary restenosis post angioplasty. Thus, we investigated the genotype distribution and relative allele frequencies of the single nucleotide polymorphisms (SNPs) rs2070744 (C>T) and rs1799983 (G>T) of the NOS3 gene in patients previously undergone successful coronary artery angioplasty in a period equal or greater than 6 months and their possible relationship with the development of restenosis.
\end{abstract}

Method: A total of 182 patients who underwent a coronary angiography and had a history of previous PTCA, at least 6 months before, were enrolled. Cases had stenosis diameter $>50 \%$ of the index lesion at follow-up angiography $(n=81)$ and controls stenosis $\leq 50 \%(n=101)$.

Demographic and clinical variables were recorded. Genotyping was performed by real-time PCR using allele-specific probes.

Results: Genotype distribution was similar between patients and controls for both polymorphisms investigated ( $r s 2070744$ and $r s 1799983, p=N S$ ). Moreover, there were no differences in the frequency of mutated alleles ( $r$ 22070744 $C$ allele: 0.30 in cases vs. 0.30 in controls, $p=0.70$; rs 1799983 T allele: 0.20 in cases vs. 0.30 in controls, $p=0.15$ ).

Conclusion: Data suggest that NOS3 gene polymorphisms, rs2070744 and rs1799983, are not associated to the development of post-angioplasty restenosis in the studied subjects.

Funding: Fondecyt \# 1141292

No conflict of interest 


\title{
234 \\ BASIC RESEARCH \\ THE ASSOCIATION BETWEEN CHOLESTEROL ESTER TRANSFER PROTEIN I405V POLYMORPHISM AND PREMATURE CORONARY ARTERY DISEASE
}

\author{
H. Goodarzynejad' ${ }^{1}$, M. Boroumand ${ }^{2}$, M. Behmanesh ${ }^{3}$, S. Ziaee ${ }^{4}$, A. Jalali ${ }^{1}$ \\ ${ }^{1}$ Tehran Heart Center, Cardiac Research, Tehran, Iran \\ ${ }^{2}$ Tehran Heart Center, Tehran, Iran \\ ${ }^{3}$ Tarbiat Modares University, Human Genetics, Tehran, Iran \\ ${ }^{4}$ Tehran Heart Center, Surgical Pathology and Laboratory Medicine, Tehran, Iran
}

Aims: The effect of human cholesteryl ester transfer protein (CETP) expression on atherogenesis is still under debate. The rs5882 (1405V) polymorphism affect CETP function. We aimed to examine the relationship between the rs5882 polymorphism and the risk of angiographically determined premature coronary artery disease (PCAD).

Method: To define PCAD, an age cutoff of 55 years for women and 45 years for men was used. A case-control study was conducted on 560 patients with newly diagnosed angiographically documented PCAD ( $\geq 50 \%$ luminal stenosis of any coronary vessel) and an equal number of control patients with normal coronary arteries (no luminal stenosis at coronary arteries). The severity of CAD was determined by vessel score and Gensini score. A real-time polymerase chain reaction (PCR) and High Resolution Melting (HRM) analysis was used to distinguish between genotypes.

Results: The 1405V genotype distributions were not statistically different in non-CAD and CAD groups in univariate and multivariable-adjusted logistic regression analyses. The median and inter-quartile range for Gensini score was not significantly different among the AA (43, 24 to 73), $A G(40,20$ to 66$)$, and $G G(45,25$ to 72$)$ genotypes (adjusted $P=0.097)$. Also, the distribution of vessel score did not statistically differ between these genotypes (adjusted $p=0.691$ ).

Conclusion: Our results suggest that there is no significant association between CETP I405V polymorphism and the risk of PCAD presence and severity. Because of the limitations of simple association studies, further larger studies are needed to investigate such association in different populations.

No conflict of interest 


\title{
BASIC RESEARCH
}

\section{PURINERGIC MODULATION IS PROTECTIVE AGAINST HYPOXIA/REOXYGENATION IN AC16 CARDIOMYOCYTES}

\author{
M. Danila ${ }^{1,2}$, L. Benoist ${ }^{3}$, S. Chadet ${ }^{3}$, C. Lefort ${ }^{3}, F$. Ivanes $^{3,4}$, D. Muntean ${ }^{1,2}$, D. Angoulvant ${ }^{3,4}$ \\ 1 "Victor Babes" University of Medicine and Pharmacy, III-Functional Sciences-Pathophysiology, \\ Timisoara, Romania \\ 2"Victor Babes" University of Medicine and Pharmacy, \\ Center for Translational Research and Systems Medicine, Timisoara, Romania \\ ${ }^{3}$ Université François-Rabelais de Tours- 37032 Tours- France, EA 4245 Cellules Dendritiques- \\ Immunomodulation et Greffes, Tours, France \\ ${ }^{4}$ Hôpital Trousseau- Centre Hospitalier Régional Universitaire de Tours- 37044 Tours- France, \\ Service de Cardiologie, Tours, France
}

\begin{abstract}
Introduction: High ATP concentrations released in the extracellular environment secondary to reperfusion injury act as signaling molecules activating cardiomyocyte purinergic receptors with subsequent modulation of cardiac inotropic and chronotropic properties. The aim of the study was to assess the effects of purinergic modulation on AC16 cardiomyocytes viability after hypoxia/reoxygenation $(\mathrm{H} / \mathrm{R})$.
\end{abstract}

Material and Methods: Cultured AC16 cardiomyocytes were subjected to hypoxia for 5 hours either in a hypoxic chamber or by mineral oil layering. Control AC16 cardiomyocytes were kept for the same duration in a normoxic incubator. During the 1 hour reperfusion, both control and $H / R$ cardiomyocytes received no additional intervention or were treated with $10 \mu \mathrm{M}, 100 \mu \mathrm{M}$ or $1 \mathrm{mM}$ ATP, respectively, or $100 \mu \mathrm{M}$ suramin (purinergic inhibitor) together with each of the above mentioned ATP additions. Cellular viability was evaluated by MTT colorimetric assay and expressed relative to the absorbance of the non-treated cells.

Results: Administration of $100 \mu \mathrm{M}$ ATP induced a significant improvement of relative cell viability $(p<0.001)$ only in the $H / R$ experiments using the hypoxic chamber, effect which was completely inhibited by suramin. Treatment with $1 \mathrm{mM}$ ATP and $1 \mathrm{mM} \mathrm{ATP}+100 \mu \mathrm{M}$ suramin elicited a significant increase of relative cell viability in control and $\mathrm{H} / \mathrm{R}$ experiments using both methods of hypoxia induction.

Conclusion: Purinergic stimulation at reoxygenation is cardioprotective both in hypoxic and control conditions, the effect being dependent on ATP concentration and the severity of the hypoxic insult. Research supported by the PII-C3-TC-2015-15132-06 research grant and by the Fédération Française de Cardiologie.

No conflict of interest 


\title{
BASIC RESEARCH
}

\section{INTRAVENOUS XENOGENEIC TRANSPLANTATION OF HUMAN ADIPOSE-DERIVED STEM CELLS IMPROVES LEFT VENTRICULAR FUNCTION AND MICROVASCULAR INTEGRITY IN SWINE MYOCARDIAL INFARCTION MODEL}

\author{
S.J. Hong, D.S. Lim \\ Korea University Anam Hospital, Cardiology, Seoul, Korea
}

\begin{abstract}
Aims: The potential for beneficial effects of adipose-derived stem cells(ASCs) on myocardial perfusion and left ventricular dysfunction in myocardial ischemia(MI) has not been tested following intravenous delivery.
\end{abstract}

Method: Surviving pigs following induction of MI were randomly assigned to 1 of 3 different groups: the placebo group ( $n=7)$, the single bolus group (SB) $\left(n=7,15 \times 10^{7} A S C s\right)$, or the divided dose group $(\mathrm{DD})\left(\mathrm{n}=7,5 \times 10^{7} \mathrm{ASCs} /\right.$ day for three consecutive days). Myocardial perfusion defect area and coronary flow reserve (CFR) were compared during the 28-day follow-up. Also, serial changes in the absolute number of circulating $C D 4^{+} T$ and $C D 8^{+} T$ cells were measured.

Results: The increases in ejection fraction were significantly greater in both the SB and the DD groups compared to the placebo group $(5.4 \pm 0.9 \%, 3.7 \pm 0.7 \%$, and $-0.4 \pm 0.6 \%$, respectively), and the decrease in the perfusion defect area was significantly greater in the SB group than the placebo group $(-36.3 \pm 1.8$ and $-11.5 \pm 2.8)$. CFR increased to a greater degree in the SB and the $\mathrm{DD}$ groups than in the placebo group $(0.9 \pm 0.2,0.8 \pm 0.1$, and $0.2 \pm 0.2$, respectively). The circulating number of CD8+T cells was significantly greater in the SB and DD groups than the placebo group at day $7(3,687 \pm 317 / \mu \mathrm{L}, 3,454 \pm 787 / \mu \mathrm{L}$, and $1,928 \pm 457 / \mu \mathrm{L}$, respectively). The numbers of small vessels were significantly greater in the SB and the DD groups than the placebo group in the peri-infarct area.

Conclusion: Both intravenous SB and DD delivery of ASCs are effective modalities for the treatment of $\mathrm{MI}$ in swine. Intravenous delivery of ASCs, with its immunomodulatory and angiogenic effects, is an attractive noninvasive approach for myocardial rescue.

No conflict of interest 
237

BASIC RESEARCH

\section{GENETIC PROFILE OF HYPERTROPHIC CARDIOMYOPATHY IN TUNISIAN PATIENTS}

N. Jaafar

Faculty of Medicine - University of Monastir, Biochemistry Laboratory- Lab-NAFS 'Nutrition Functional Food and Vascular Health', Monastir, Tunisia

Aims: Hypertrophic Cardiomyopathy $(\mathrm{HCM})$ is a common inherited heart disease, caused by mutations in genes encoding for sarcomere proteins and transmitted in an autosomal dominant form. However, the genetic bases of HCM in Tunisia has not previously explored.

Method: Next Generation Sequencing (NGS) genetic screening has been performed in 11 consecutive and unrelated Tunisian HCM probands. Using the Illumina platform, a panel of 12 genes was analyzed including myosine binding protein C (MYBPC3), beta-myosin heavy chain (MYH7), regulatory and essential light chains (MYL2 and MYL3), troponin-T (TNNT2), troponin-I (TNNI3), troponin-C (TNNC1), alpha-tropomyosin (TPM1), alpha-actin (ACTC1), alpha-actinin-2 (ACTN2) as well as alpha-galactosidase (GLA), 5'-AMP-activated protein (PKRAG2), transthyretin (TTR) and lysosomal-associated membrane protein-2 (LAMP2) for exclusion of phenocopies.

Results: 8 mutations were identified in 5 of the 11 patients (45\%). Of the 5 genotype-positive, one patient had a mutation in MYH7, one in MYBPC3, one in MYL3, one was a TNNC1/ACTN2 double mutant and one in MYL2/TNNT2.

Conclusion: Despite the limitations inherent to the small sample size, HCM in Tunisia may have a peculiar genetic background which privileges rare genes overs the classic $\mathrm{HCM}$-associated MHY7 and MYBPC3 genes. This hypothesis deserves further investigation, as it may importantly impact the epidemiology, phenotypic expression and severity of the disease in Tunisia, including predisposition to sudden cardiac death.

No conflict of interest 


\title{
BASIC RESEARCH
}

\section{UTILIZING THE INFORMATION NEEDS IN CARDIAC REHABILITATION TOOL FOR CARDIAC REHABILITATION PATIENTS: DOES ONE SIZE FIT ALL?}

\author{
K. Hassan ${ }^{1}$, V. Valle', R. Francia², R. Shaw ${ }^{1}$ \\ ${ }^{1}$ California Pacific Medical Center, Cardiology, San Francisco, USA \\ ${ }^{2}$ California Pacific Medical Center, Cardiac Rehabilitation, San Francisco, USA
}

\begin{abstract}
Aims: Assess information delivery preferences and needs in cardiac rehabilitation patients using the Information Needs for Cardiac Rehab Patients (INCR) survey tool.
\end{abstract}

Method: Data were collected using the validated INCR, which was offered to all patients referred for cardiac rehabilitation at California Pacific Medical Center (CPMC) between November 2014 and August 2015. Sixty random patients voluntarily completed the survey before beginning the program. The INCR is comprised of 55 'information needs' items with five subscale categories. Each item is scored on a 5-point Likert scale, with 1 being the least and 5 being most important. The survey also includes 7 information delivery format questions and a section for comments.

Results: The overall response score distribution of the patients (mean age $=65.1,71.1 \%$ males) differed in various categories. On average, patients rated learning about their medications highest (4.26) whereas support systems were rated lowest (3.57). Learning about medication interactions was rated a 5 by $68.3 \%$ of patients. Learning about smoking was rated low, with $33.9 \%$ scoring it a 1 . Most $(68 \%)$ preferred discussion with a health care provider, while lecture format was selected the least (15\%) out of the preferred information delivery system options.

Conclusion: Prior studies support comprehensive health information needs assessment as a first step in developing patient educational programs. This study suggests the need for individualized patient negotiated cardiac teaching programs to address individual patient needs. The study also supports the use of a routine pre-enrollment survey to assess patient needs and identify the most suitable information delivery system.

No conflict of interest 


\title{
BASIC RESEARCH
}

\section{ADDRESSING HYPERTENSION CARE GAPS BY IMPLEMENTING AN EVIDENCE BASED ELECTRONIC MEDICAL RECORD (EMR) HYPERTENSION DASHBOARD}

\author{
M. Mann, M. Qiu, D. Ngui \\ Fraser Street Medical, Vancouver, Canada
}

\begin{abstract}
Aims: In primary care, it can be challenging to systematically identify and recall patients with care gaps based on our current national hypertension guidelines. However, with increased adoption and meaningful use of EMRs, chronic disease care can be made substantially easier and efficient. Our quality improvement project's objective was to develop a real time EMR tool based on the 2014 CHEP (Canadian Hypertension Education Program) Guidelines.
\end{abstract}

Method: We reverse engineering selected evidence based recommendations to create widgets. We were able to create 9 widgets based on Grade A and B level evidence to identify a total of 1084 patients eligible for population health management.

Results: Also, we held both individual and focus group sessions for clinicians at our clinic and utilized a formal worksheet to identify the top enablers to use which included:

1. Improved patient care

2. Increased knowledge of current guidelines

3. Improved sustainablepractice management and opportunity for delegation for team based care

We discovered the top barriers to use which included:

1. Time Barriers to use in daily practice

2. Need to learn how to use these new electronic tools

3. Concerns regarding over treatment when focused on numbers

Additionally, we gathered feedback on clinician's design preferences that would optimize theuse of the dashboard. We were able to get feedback from $\mathrm{N}=7$ physicians, $\mathrm{N}=3$ clinical pharmacists, $\mathrm{N}$ $=1$ resident, and $\mathrm{N}=1$ medical student. Conclusion

Our prototype EMR HTN dashboard requires more study but could be a future simple to use and efficient option to improve care of hypertensive patients.

Conflict of Interest Disclosure Statement: We received an unrestricted educational grant from Mylan Pharmaceuticals. 


\title{
BASIC RESEARCH
}

\section{LONGITUDINAL STUDY OF CARDIAC MORPHOLOGICAL AND FUNCTIONAL ADAPTATIONS IN RATS SUBJECTED TO PHYSIOLOGICAL AND EARLY OVARIAN FAILURE}

\author{
A.C. Sanches Felix, S.G. Veloso Dutra, H.C. Dutra de Souza \\ Faculty of Medicine of Ribeirao Preto, Physiology, Ribeirão Preto- São Paulo, Brazil
}

Aims: Evaluate and compare longitudinally the cardiac morphofunctional changes caused by physiological and early ovarian failure associated to aging process.

Method: Wistar rats with physiological (SHAM, N=8) and early (OVX, N=8) ovarian failure were submitted to Echocardiography in the $22^{\text {nd }}, 42^{\text {nd }}, 62^{\text {nd }}$ and $72^{\text {nd }}$ wk age. Statistical Analysis: OneWay ANOVA, post-test Student Newman-Keuls test. Ethics Committee: 178/2011.

Results: SHAM group showed lowest posterior wall thickness (PWT), interventricular septum (IVST), relative wall thickness (RWT) of the left ventricle (LV) and LV mass at the 42nd, 62nd and 72nd wk age, in addition to lowest ejection fraction (EF), fractional shortening (FS) and endsystolic volume (LVESV) at the 62nd and 72nd wk age when compare to 22nd wk age. OVX group presented lowest PWT, IVST, RWT, LV mass, systolic diameter (LVESD) and end-diastolic LV (LVEDD) at the 42nd and 62nd wk age and increase of heart rate (HR), volume ejection and cardiac output at the $42 n d, 62 n d$ and $72 n d$ wk age when compared to 22 nd wk age. Comparisons between OVX and SHAM groups in the same age showed that OVX group presented lowest PWT, IVST, LVEDD, LV mass, HR, EF, FS, cardiac output and index at the 22nd wk age; lowest PWT, LV mass and bigger stroke volume and cardiac output at the 42nd wk age and finally, lowest IVST, RWT and LV mass at the 62nd and 72nd wk age.

Conclusion: Both groups showed cardiac morphological changes over the aging process, suggesting that ovarian hormones can influence on cardiac remodeling in all stages, especially while young.

No conflict of interest 


\title{
BASIC RESEARCH
}

\section{NEUROHUMORAL ASPECT OF OBESITY AND ATHEROSCLEROSIS: LEPTIN/ADIPONECTIN RATIO AND CORONARY HEART DISEASE}

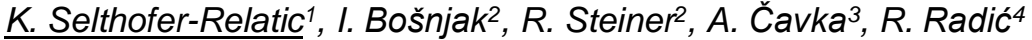 \\ ${ }^{1}$ Clinical Hospital Center Osijek, Department for Cardiovascular Disease, Osijek, Croatia \\ ${ }^{2}$ University Hospital Centre Osijek, Department for Cardiovascular Medicine, Osijek, Croatia \\ ${ }^{3}$ Faculty of Medicine- University J.J.Strossmayer, Department for Physiology and Immunology, \\ Osijek, Croatia \\ ${ }^{4}$ Faculty of Medicine- University J.J.Strossmayer, Department for Anatomy, Osijek, Croatia
}

Aims: Obesity related atherosclerosis is related to acute and chronic cardiovascular disease. Endothelial dysfunction and inflammation are the key point for early prevention and treatment. Adipokines are part of complex obesity related neurohomoral activity or adipo-vascular axis, where increased leptin level and decreased adiponectin level results in proinflammatory, proatherogenic state. Leptin/adiponectin ratio (LAR) could potential present atherosclerotic marker where its relation with coronary heart disease and cardiac syndrome $\mathrm{X}$ is still unknown.

Method: Study included 80 adult males and females, who were hospitalized because of myocardial non-STE myocardial infarction. They were hypertensive, overweight, non-diabetic with dyslipidemia. Biochemic, anthropometric, cardiovascular measurements were done to all patients. Serum leptin level was measured by RIA test, and total adiponectin level by ELISA test.

Results: Results showed that LAR have no significant relation with significant coronary artery stenosis. It is significant related with glucose level, lipidograme and anthropometric parametars.

Conclusion: LAR could present part of complex adipo-athero-vascular axis. Together with standard risk factor is a part of unique persons atherosclerotic profile that leads in manifest coronary artery disease.

No conflict of interest 


\title{
BASIC RESEARCH
}

\section{CHRONIC TREATMENT WITH ENALAPRIL MALEATE PREVENTS THE DEVELOPMENT OF HYPERTENSION AND CARDIAC REMODELING IN OLD RATS WITH EARLY OVARIAN FAILURE}

\author{
S.G. Veloso Dutra ${ }^{1}$, A.C. Sanches Felix ${ }^{2}$, H.C. Dutra de Souza ${ }^{2}$ \\ ${ }^{1}$ School of Medicine of Ribeirão Preto - University of São Paulo, Department of Physiolgy, \\ Ribeirao Preto, Brazil \\ ${ }^{2}$ School of Ribeirão Preto - University of São Paulo, Department of Physiology, Ribeirão Preto, \\ Brazil
}

Aims: The aim of present study was to investigate the preventive effect of chronic treatment with enalapril maleate (EM) on hemodynamic, autonomic and morphofunctional cardiac changes, caused by physiological and early ovarian failure in old rats.

Method: Wistar rats (10 wk) were divided into 4 groups: physiological ovarian failure treated with water (SHAM, $\mathrm{n}=6$ ) or EM (SHAM-EM, $\mathrm{n}=8), 10 \mathrm{mg} / \mathrm{kg} / \mathrm{day}$, po, until the $82^{\text {nd }}$ week of age, and early ovarian failure treated with water (OVX, $n=7)$ or EM (OVX-EM, $n=8)$. The hemodynamic [systolic (SAP), diastolic (DAP) and mean arterial pressure (MAP); baseline (bHR) and intrinsic heart rate (iHR)]; autonomic [atropine/ propranolol, baroreflex sensitivity (BRS), heart rate variability (HRV)]; and morphofunctional parameters [echocardiography] were evaluated. Statistics: two-way ANOVA, post-test Student-Newman-Keuls. Committee ethics: 178/2011.

Results: OVX group showed higher SAP and interventricular septum thickness (IVST), also lower BRS and vagal participation in determining the bHR than the SHAM group. SHAM-EM group presented lower SAP, DAP, MAP, bHR, iHR, and sympathetic participation in determining the bHR and LFnu-HRV, in addition to a higher HFnu-HRV, absolute and relative heart weight to the SHAM group. The OVX-EM group had lower SAP, MAP, LFnu-HRV, relative thickness and posterior wall of the left ventricle (LV), IVST and LV mass, in addition to an increased vagal participation in determining the bHR, HFnu-HRV and BRS than the OVX group.

Conclusion: The physiological and early ovarian failure in old rats causes important hemodynamic, autonomic and morphological cardiac changes that can be prevented by chronic treatment with enalapril maleate.

No conflict of interest 


\title{
PARACRINE ACTIONS OF NSTEMI PATIENTS CD34-/CD31+ CACS IMPAIR HUVEC TUBE FORMATION AND ARE MEDIATED BY TLR4
}

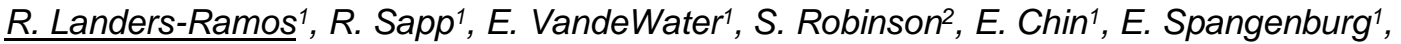 \\ S. Prior ${ }^{3}$, J. Hagberg ${ }^{1}$ \\ ${ }^{1}$ University of Maryland, Kinesiology, College Park, USA \\ ${ }^{2}$ Baltimore Veterans Affairs Medical Center, Cardiology, Baltimore, USA \\ ${ }^{3}$ University of Maryland School of Medicine and Veterans Affairs Medical Center, GRECC, \\ Baltimore, USA
}

Aims: Conditioned medium (CM) from CD34-/CD31+ circulating angiogenic cells (CACs) of
inactive but otherwise healthy, younger adults impairs human umbilical vein endothelial cell
(HUVEC) tube formation compared to their endurance exercise trained counterparts. Higher
levels of S100A8/A9 in the CM of inactive individuals' CACs appear to be mediating these
differences. We aimed to determine if CM derived from CACs of non ST-segment elevation
myocardial infarction (NSTEMI) patients results in impaired tube formation and whether pre-
treatment of HUVECs with inhibitors for major receptors of S100A8/A9 will improve tube
formation to levels similar to those observed when using CM from endurance-trained athletes.

Method: Blood was drawn from young endurance-trained athletes and NSTEMI patients ( $n=10 /$ group) and CD34-/CD31 + CACs were isolated. CACs were cultured for 48hrs and CM was used in a HUVEC-based angiogenesis assay with and without pretreatment with the TLR4 inhibitor, TAK-242, or RAGE antagonist, FPS-ZM1.

Results: CM derived from NSTEMI patient CACs resulted in $9 \%$ lower total tube length $(\mathrm{P}<0.05)$ and $18 \%$ fewer nodes $(\mathrm{P}=0.08)$ compared to $\mathrm{CM}$ from endurance-trained athletes but number of tube meshes or branches was not significantly different $(P>0.05)$. Pre-treatment with TAK-242, but not FPS-ZM1, significantly improved total tube length $(P<0.05)$, number of branches $(P<0.05)$ and number of meshes $(\mathrm{P}<0.05)$ in the NSTEMI patients compared to $\mathrm{CM}$ alone. Treatments did not affect tube formation in the endurance-trained cell CM conditions $(P>0.05)$.

Conclusion: Factors present in CM from CD34-/CD31+ CACs of NSTEMI patients impair HUVEC tube formation. These effects appear to be mediated through TLR4, a major receptor for S100A8/A9.

No conflict of interest 


\title{
244
}

ANGIOGENESIS, GENE THERAPY

\section{DECELLULARIZED PORCINE PERICARDIUM FOR CARDIOVASCULAR TISSUE ENGINEERING}

\author{
M. Markova ${ }^{1}$, E. Filova' ${ }^{1}$ M. Konarik ${ }^{2}$, J. Pirk' ${ }^{2}$, E. Matejkova ${ }^{3}$, L. Bacakova ${ }^{1}$ \\ ${ }^{1}$ Institute of Physiology of the Czech Academy of Sciences, \\ Department of Biomaterials and Tissue Engineering, Prague, Czech Republic \\ 2Institute for Clinical and Experimental Medicine, Cardiology Department, Prague, \\ Czech Republic \\ ${ }^{3}$ National Tissue Centre Inc., Cell Therapy Department/Cord Blood Banking, Prague, \\ Czech Republic
}

Aims: Biological prostheses made of decellularized cross-linked bovine pericardium and porcine aortic heart valves or mechanical prostheses are used for transplantation of aortic heart valves. None of these prostheses contain autologous cells.

The purpose of our study is to prepare decellularized porcine blood vessels and porcine pericardium, which would be suitable for colonizing with both autologous stem cells and endothelial cells, and subsequently transplanted into a patient.

Method: For decellularization, we tested different solutions and time of exposure. Effectiveness of decellularization process was assessed by hematoxylin-eosin staining and Hoechst staining.

Results: We managed to prepare decellularized tissues.

Conclusion: These materials will be further evaluated for their biocompatibility and will be seeded with bone marrow-derived stem cells or adipose tissue stem cells and with endothelial cells.

Acknowledgement. Supported by the Ministry of Health of the Czech Republic, AZV CR agency, project No. 15-29153A, by the Grant Agency of the Czech Republic, project No. P108/12/1168, Technology Agency of the Czech Republic, Project No. TA04011345 and by BIOCEV Biotechnology and Biomedicine Centre of the Academy of Sciences and Charles University project (CZ.1.05/1.1.00/02.0109), funded by the European Regional Development Fund.

No conflict of interest 


\title{
245
}

\section{ANGIOGENESIS, GENE THERAPY}

\section{POLYPHENOLS INHIBIT IN VITRO ANGIOGENESIS THROUGH MODULATION OF MICRORNAS EXPRESSION}

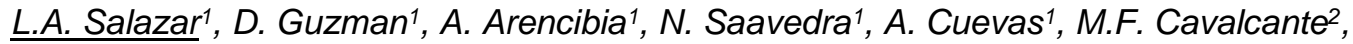 \\ D.S.P. Abdalla ${ }^{2}$ \\ ${ }^{1}$ Universidad de La Frontera, Center of Molecular Biology \& Pharmacogenetics, Temuco, Chile \\ ¿Universidade de São Paulo, Departamento de Analises Clínicas e Toxicológicas, São Paulo, \\ Brazil
}

\begin{abstract}
Aims: Polyphenols have been emerged as modulators of several biological processes, including angiogenesis that plays an important role in promoting the instability of atherosclerotic plaques. However, the molecular mechanisms involved in antiangiogenic activity of these compounds remain poorly understood. Thus, we aimed to evaluate the effect of treatment with polyphenolrich extract of propolis and some of its main constituents on the expression of angiogenesisrelated miRNAs using in vitro models.
\end{abstract}

Method: MTT assay was used to select a concentration of polyphenols-rich extract of propolis, pinocembrin, pinobanksin and caffeic acid treatments that do not alter the cell viability. Then, the antiangiogenic potential of treatments was evaluated by assessing the formation of capillary-like structures and the migration of endothelial cells. Finally, bioinformatic approaches were used to identify those miRNAs that can regulate angiogenesis-related pathways, from which miR155, miR-126, miR-21 and let-7b were selected and quantified by qPCR.

Results: Viability assay showed that cell treated with $20 \mu \mathrm{g} / \mathrm{mL}$ of polyphenols maintained the cellular viability. Regarding the formation of capillary-like structures, cells treated with polyphenolrich extract of propolis, pinocembrin and pinobanksin showed a lesser angiogenic-ratio. In contrast, the migration of endothelial cells was only affected by pinobanksin treatment. Finally, the quantification of miRNAs showed that miR-155 and miR-21 were down-regulated under caffeic acid and pinocembrin treatments, respectively.

Conclusion: Our results showed that polyphenols reduce in vitro angiogenesis by modulating the expression of angiogenesis-related miRNAs depending on the polyphenol used as treatment, suggesting that each polyphenol contribute to antiangiogenic effect of propolis by modulating different molecular pathways.

No conflict of interest 


\title{
ANGIOGENESIS, GENE THERAPY
}

\section{THE CURRENT STATUS OF GENE-BASED THERAPEUTIC ANGIOGENESIS FOR PERIPHERAL ARTERIAL DISEASE}

\author{
M. Tanaka, Y. Yoshikazu \\ Kyushu University, Laboratory for Innovative Biotherapeutics Science, Fukuoka, Japan
}

Aims: Peripheral artery disease (PAD) is a highly prevalent disease, which still has unmet medical needs. Therapeutic angiogenesis for PAD, achieved by gene therapy, has achieved promising results. This study reviewed revealed current status and provides an overview of the progression of angiogenesis research in gene therapy field as it applies to PAD.

Method: The focus of angiogenic growth factors and clinical trials are introduced as a frontier of therapeutic angiogenesis and also includes insights into future directions from bench to bedside.

Results: Proangiogenic growth factors such as VEGF, FGF, HGF, HIF, and DEL have demonstrated angiogenic effects in PAD. The isuues of boundaries in gene therapy approaches were aroused such as safety, trial design, type of vector, duration of treatment, administration route, and dose.

\begin{tabular}{|c|c|c|c|c|c|c|c|c|}
\hline Patients & $\begin{array}{c}\text { No. of } \\
\text { subjects } \\
\text { (treated co } \\
\text { ntrols) }\end{array}$ & $\begin{array}{l}\text { Angiogenic } \\
\text { factor }\end{array}$ & Treatment & Vector & Route & $\begin{array}{c}\text { Follow- } \\
\text { up }\end{array}$ & Primary endpoints & Outcome* \\
\hline \multicolumn{9}{|l|}{ Phase I } \\
\hline CLI & $\%$ & VEGF & PCK-VEGF is & Plasmid & Intramuscular & $9 \mathrm{mo}$ & Safety & Positive \\
\hline CLI & $21 / \cdot$ & VEGF & phVEGF $_{\text {is }}$ & Plasmid & Intramuscular & $6 \mathrm{mo}$ & Safety & Positive \\
\hline IC & $15 / 3$ & VEGF & $\operatorname{adVEGF}_{13}$ & Adenovirus & Intramuscular & $12 \mathrm{mo}$ & Safety & Equivocal \\
\hline CLI & $51 /$. & FGF.1 & NVIFGF & Plasmid & Intramuscular & $6 \mathrm{mo}$ & Safety & Positive \\
\hline CLI & $12 /$. & FGF-2 & rSeV/df-hFGF-2 & Sendai vinus & Intramuscular & $6 \mathrm{mo}$ & safety and tolerability & Positive \\
\hline CLI & $78 / 26$ & HGF & pVAXI-HGF & Plasmid & Intramuscular & $12 \mathrm{mo}$ & Safety/TcPO2 & Positive \\
\hline $\mathrm{CLI}$ & $22 /$. & HGF & PVAXI-HGF & Plasmid & Intramuscular & $6 \mathrm{mo}$ & Safety/ABI, ulcer size & Positive \\
\hline CLI & $21 / \cdot$ & HGF & $\mathrm{PCK} \cdot \mathrm{HGF} \cdot \mathrm{X} 7$ & Plasmid & Intramuscular & $3 \mathrm{mo}$ & Safety and tolerability & Positive \\
\hline CLI & 3477 & $\mathrm{HIF}=\mathrm{I}$ & $\mathrm{Ad} 2 / \mathrm{HIF}=1 \mathrm{a} \mathrm{VP} 16$ & Adenovirus & Intramuscular & $12 \mathrm{mo}$ & Safery and efficacy & Positive \\
\hline \multicolumn{9}{|l|}{ Phase II } \\
\hline $\begin{array}{l}\text { Diabetes } \\
\text { with CLI }\end{array}$ & $27 / 27$ & VEGF & phVEGF $_{\text {is }}$ & Plasmid & Intramuscular & $100 \mathrm{~d}$ & Amputation & Negative \\
\hline IC & $72 / 33$ & VEGF & $\operatorname{adVEGF}_{13}$ & Adenovinus & Intramuscular & $26 \mathrm{wk}$ & Peak walking time & Negative \\
\hline IC/CLI & 18/17/19 & VEGF & $\begin{array}{l}\text { adVEGF is/ } \\
\text { phVEGF }_{\text {iss }}\end{array}$ & $\begin{array}{l}\text { Adenovirus' } \\
\text { plasmid }\end{array}$ & Intraarterial & $3 \mathrm{mo}$ & Increased vascularity & Positive \\
\hline CLI & $51 / 56$ & FGF.1 & NVIFGF & Plasmid & Intramuscular & 25 wk & Ulcer healing & Negative \\
\hline IC & $127 / 63$ & FGF-2 & $\mathrm{IFGF} 2$ & Plasmid & Intraarterial & $90 \mathrm{~d}$ & Peak walking time & Positive \\
\hline CLI & $21 / 6$ & HGF & VM202 & Plasmid & Intramuscular & $12 \mathrm{mo}$ & Safery/TBI, VAS, ulcer & Positive \\
\hline CLI & $213 / 76$ & HIF-I & $\mathrm{Ad} 2 / \mathrm{HIF}-\mathrm{la} \mathrm{VP} 16$ & Adenovinus & Intramuscular & $12 \mathrm{mo}$ & Peak walking time & Negative \\
\hline IC & $52 / 53$ & $\mathrm{DEL}-\mathrm{I}$ & VLTS-589 & Plasmid & Intramuscular & $180 \mathrm{~d}$ & Peak walking time & Negative \\
\hline \multicolumn{9}{|l|}{ Phase III } \\
\hline CLI & $259 / 266$ & FGF-1 & NVIFGF & Plasmid & Intramuscular & $12 \mathrm{mo}$ & Amputation of death & Negative \\
\hline CLI & $30 / 14$ & $\mathrm{HGF}$ & pVAXI-HGF & Plasmid & Intramuscular & $15 \mathrm{mo}$ & Rest pain, ulcer size & Positive \\
\hline
\end{tabular}

Conclusion: Despite success of proangiogenic gene therapy in preclinical studies and earlyphase clinical trials, clinical benefits still do not fulfill expectations. To overcome failure from bench to bedside, gene therapy is still waiting for large phase III clinical trials and long-term safety evaluation. 
No conflict of interest 


\title{
247 \\ INTERVENTIONAL AND STENTING
}

\section{TRANSBRACHIAL COIL EMBOLIZATION OF A GIANT CORONARY ARTERY FISTULA}

\author{
T. Kuroyanagi ${ }^{1}$, T. Komatsu ${ }^{1}$, A. Fujikake', S. Komatsu' ${ }^{1}$, H. Nakamura ${ }^{1}$, A. Higashi', \\ A. Nakagawa ${ }^{1}$, Y. Sakai', I. Taguchi ${ }^{1}$ \\ ${ }^{1}$ Dokkyo Medical University Koshigaya Hospital, Cardiology, Koshigaya City, Japan
}

Aims: A 71-year-old woman visited our hospital because of chest pain. Computed tomography revealed right coronary dilatation and a giant fistula $(6 \times 6 \times 12 \mathrm{~cm})$. The fistula drained into the coronary sinus. Coronary angiography demonstrated that the left coronary artery was normal, but the right coronary artery (RCA) showed severe dilatation and confirmed the presence of the giant fistula.

Method: We tried trans-brachial coil embolization. Our procedure inserted a 6-Fr sheath in the right brachial artery. A 4.5-Fr straight guiding catheter was used to enter the right coronary ostium, and was deeply engaged to access the mid RCA. IDC embolization coils and Diamond embolization coils were delivered to the distal vessel via a micro catheter. We were careful to avoid the lumen of the posterior descending artery. This resulted in the occlusion of the fistulous communication and the posterior descending artery was confirmed to have excellent flow. The patient's hemodynamics remained stable during the procedure, and there were no ECG changes indicative of myocardial ischemia. The follow-up coronary angiography performed at 10 months confirmed complete closure of the RCA fistula.

Results: Anomalies of the coronary arteries are uncommon. Congenital coronary arteriovenous fistula constitutes $0.87 \%$ of all coronary artery anomalies. Coil embolization and trans-brachial approach are very easy, safe and less invasive than other methods. In our patient, percutaneous closure produced long-term results.

Conclusion: With this case we have demonstrated that a giant coronary fistula can be closed by coil embolization following the trans-brachial approach and using a small size catheter.

No conflict of interest 
248

\title{
INTERVENTIONAL AND STENTING
}

\section{SUCCESSFUL PERCUTANEOUS CORONARY THROMBOLYTIC THERAPY OF MYOCARDIAL INFARCTION CAUSED BY CABROL CONDUIT GRAFT}

\author{
T. Kuroyanagi', T. Komatsu ${ }^{1}$, A. Fujikake ${ }^{1}$, S. Komatsu ${ }^{1}$, H. Nakamura ${ }^{1}$, A. Higashi', \\ A. Nakagawa ${ }^{1}$, Y. Sakai', I. Taguchi ${ }^{1}$ \\ ${ }^{1}$ Dokkyo Medical University Koshigaya Hospital, Cardiology, Koshigaya City, Japan
}

Aims: A 66-year-old man presented with new chest pain. He reported a Bentall operation 5 years ago but did not know additional details. Because ECG shows ST elevation in II, III, aVF leads, the patient underwent coronary angiography.

Method: An initial aortography demonstrated the presence of a Cabrol composite graft, with a steep downward course of the 2 limbs of the coronary conduit. Contrast injection into the left limb of the conduit demonstrated filling of the left coronary artery. But the right coronary artery was found occluded at the site of the end-to-end anastomosis with the right graft limb. The right coronary artery was not reopened with a balloon dilatation because of a lot of thrombus. So we injected $400000 \mathrm{IU}$ of monteplase to coronary artery. When we underwent coronary angiography 2 days ago, the thrombus had disappeared completely.

Results: According to Cabrol, the coronary ostium was end-to-end anastomosed to a second Dacron tube $10 \mathrm{~mm}$ in diameter and $80 \mathrm{~mm}$ long, situated to the right of the ascending aortic graft and anastomosed side-to-side. The Cabrol technique is used only rarely but provides good longterm results. But some cases needed management by PCI.

Conclusion: One of important causes of this management is acute thrombosis of the coronary grafts. This can be one of the causes of early onset sudden death. However, there have been no reported cases that have been managed by $\mathrm{PCl}$ in acute coronary graft thrombosis. Antithrombolytic therapy of massive thrombus is important for possible coronary intervention.

No conflict of interest 


\title{
INTERVENTIONAL AND STENTING
}

\section{LONG TERM FOLLOW UP OF TRANSCATHETER AORTIC VALVE IMPLANTATION IN CIRRHOTIC PATIENTS WITH SEVERE AND SYMPTOMATIC AORTIC STENOSIS}

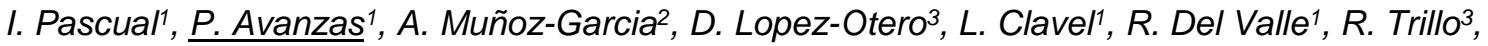 \\ J.M. Hernandez ${ }^{2}$, C. Morís ${ }^{1}$ \\ ${ }^{1}$ Hospital Central de Asturias, Cardiology, Oviedo, Spain \\ ${ }^{2}$ Hospital Universitario Virgen de la Victoria., Cardiology, Malaga, Spain \\ ${ }^{3}$ Complejo Hospitalario Universitario de Santiago, Cardiology, Santiago de Compostela, Spain
}

Aims: Cirrhosis is not included into preoperative scales of risk but cirrhotic patients who undergo to cardiac surgery have high risk of morbidities and mortality related to surgery.

The main objective of this study is to describe the efficacy and security of TAVI in cirrhotic patients with severe and symptomatic aortic stenosis.

Method: Multicenter, observational and prospective study carried out in 3 hospitals. We included consecutive patients with cirrhosis with severe and symptomatic AS treated with TAVI. The primary endpoint was to evaluate death from any cause at 2 years.

Results: Ten patients were included. Cirrhosis classification grades were: 7 patients Child A, 2 Child $B$ and 1 Child $C$. Nine patients were treated under deep sedation and only one under general anesthesia. Femoral approach was used in all cases. During the procedure, there was a mayor bleeding complication due to vascular perforation of femoral artery that was completely solved by the implantation of a covered stent. In hospital stay was $10,4+6,5$ days. There wasn't deaths during the hospital stay period. During the follow up period (median 1031 days, IQ range 268-1737) there were four deaths. One patient received liver transplantation after the TAVI. Only one of the deaths was due to cardiac cause after 250 days of follow up. Global survival was $60 \%$ (figure) with a mean estimate survival time 1373,5 days (IC 95\%, 822,6-1924,4) 


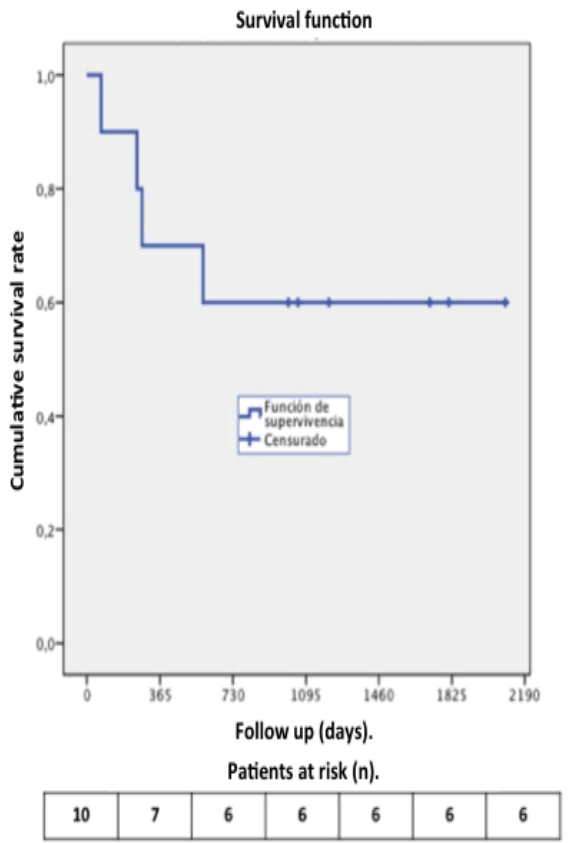

Conclusion: TAVI could be a safe and secure alternative treatment for cirrhotic patients, even as a previous step before liver transplantation, with severe and symptomatic aortic stenosis.

No conflict of interest 


\title{
INTERVENTIONAL AND STENTING
}

TRANSRADIAL APPROACH FOR CORONARY ANGIOGRAPHY AND INTERVENTIONS: A CLINICAL NURSE SPECIALIST'S EXPERIENCE

\author{
V. Perovic, D. Sagic, S. Vucinic \\ Institute of cardiovascular diseases, Cath Lab, Belgrade, Serbia
}

Aims: Transradial approach (TRA) for coronary angiography and percutaneous coronary interventions $(\mathrm{PCl})$ has risen gradually last decade. Last few years there is increasing number of reports and new published studies providing further support for the transradial approach in diagnostic and coronary $\mathrm{PCl}$. This route provides patient comfort and lower rates of vascular complications.

To demonstrate safety and advantage of this access in diagnostic and interventions, not only for electrive patients, but for those in complex and with ACS and AMI primary interventions as well.

Method: We analysed 1032 patients who underwent TRA diagnostic and/or $\mathrm{PCl}$ interventions in our Cath.lab. 15.01.2014. - 15.06.2014.

Results: There were 426 patients with $\mathrm{PCl}$ and 606 with diagnostic procedures only. Rate of radial access was almost $95 \%$, wheras procedural succes rate was as high in both groups $90 \%$ and $92 \%$ respectively. Selective cannulation of the coronary ostium failed in only $2,5 \%$. There were no bleeding complications need for surgical repair or blood transfusions and no other complications in any group as well. We are presenting all procedural dates, and in three typical cases, one with diagnostic only, the second with elective complex intervention, and finally the third with serious ACS snf STEMI interventions, to demonstrate our strategy, technical preferences and adventages of TRA access approach.

Conclusion: As a Clinical Nurse Specialist in Cardiology the key element of my clinical role is to ensure quality of care and improved continuity, from making the patient transition of an Emergency situation. Within our Cardiology center Radial access has become our first preference.

No conflict of interest 


\title{
251
}

\section{INTERVENTIONAL AND STENTING}

\section{A USEFUL DEVICE TO DELIVER A STENT IN CALCIFIED TORTUOUS CORONARY LESIONS}

\author{
K. Saito, Y. Uchida, I. Ishida, K. Yoshida, A. Kim, K. Jujo \\ Nishiarai Heart Center Hospital, Department of Cardiology, Tokyo, Japan
}

\begin{abstract}
Aims: PCI (percutaneous coronary intervention) technique has been established as a strong therapeutic method for coronary artery disease. Especially, implantation of drug eluting stents has fulfilled a central role in PCI technique. However, we sometimes experience that it is very difficult to deliver a drug eluting stent in calcified tortuous coronary arteries. The aim of this study is to examine usefulness of "Guideliner" as an adjunctive device to deliver a stent to a target lesion.
\end{abstract}

Method: In consecutive PCl cases, the usefuleness of 'Guideliner" for tough lesions to delivery a stent was examined.

Results: We performed tconsecutive $192 \mathrm{PCl}$ cases from 1st January to 31st August 2015. Average age of the cases was 69.7 years old and males were 134 (69.8\%). Guideliners (Vascular Solutions) were used in 16 cases during the period because of difficulty in delivering drug eluting stents after dilatation with balloon catheters in calcified tortuous coronary arteries. The 16 cases were 10 males and 6 females and the average age was 70.9 years old. Eight lesions were in the right coronary artery, five were in the left anterior descending artery and 3 were in the left circumflex artery. In all the cases, we succeed in delivering and implanting drug eluting stents adequately in the target lesions.

Conclusion: Guideliner catheter may become one of useful devices to overcome failure of delivery drug eluting stents in calcified tortuous coronary lesions.

No conflict of interest 
252

\title{
INTERVENTIONAL AND STENTING
}

\section{MANUAL HEATING OF RADIAL ARTERY TO FACILITATE RADIAL PUNCTURE PRIOR TO TRANSRADIAL CORONARY CATHETERIZATION: A RANDOMIZED, DOUBLE-BLIND CLINICAL TRIAL. THE BALBAY MANEUVER STUDY}

\author{
S. Ünal', B. Açar', M. Kara', M.M. Balci', O. Maden'1, A. Temizhan'1, Y. Balbay ${ }^{1}$, M. Tola ${ }^{2}$ \\ ${ }^{1}$ Türkiye Yüksek Ihtisas Education And Research Hospital, Cardiology, Ankara, Turkey \\ ${ }^{2}$ Türkiye Yüksek Ihtisas Education And Research Hospital, Radiology, Ankara, Turkey
}

\begin{abstract}
Aims: Transradial access (TRA) is being increasingly used for both diagnostic and interventional cardiac procedures. Use of TRA offers many advantages: decreased bleeding, vascular complications, reduced length of hospital stay, and reduced cost. However, the small size of the radial artery limits the size of the equipment that can be used via this approach. We sought to investigate that pre-procedural manual heating of radial artery is facilitates radial artery puncture.
\end{abstract}

Method: Patients undergoing transradial cardiac catheterization were randomized in a doubleblind fashion to a subcutaneous combination of nitroglycerin+diltiazem or manual heating (Balbay Maneuver). The study endpoint was puncture score( score 1: easiest puncture - first try, score 2: puncture at second try, score 3:puncture at third try, score 4: puncture at forth or more try, score 5: radial puncture failed).

Results: 90 patients were enrolled (45 allocated to treatment group and 45 to heating group). Patients underwent ultrasound of the radial artery before the catheterization. Complications were rare: one hematoma (treatment group) and one radial artery occlusion (heating group). Baseline demographic and clinical characteristics were similar. The baseline radial artery diameter was similar in both groups. $(2.41 \pm 0.46 \mathrm{~mm}$ in heating group and $2.30 \pm 0.48 \mathrm{~mm}$ in treatment group. However, puncture score was $1.47 \pm 0.9$ in heating group and $2.22 \pm 1.2$ in treatment group $(\mathrm{p}=0.002)$, respectively.

Conclusion: Pre-procedural manual heating of radial artery by BALBAY maneuver is facilitates radial artery puncture in patients undergoing transradial cardiac catheterization.

No conflict of interest 
253

INTERVENTIONAL AND STENTING

\title{
USEFULNESS OF NGAL AND KIM-1 AS EARLY BIOMARKERS OF CONTRAST-INDUCED NEPHROPATHY AFTER PERCUTANEOUS CORONARY INTERVENTION
}

\author{
K. Zbierska, I. Chrzan, O. Pańkiw, A. Gackowski \\ Jagiellonian University Medical College, \\ Department of Coronary Artery Disease and Heart Failure, Kraków, Poland
}

\begin{abstract}
Aims: The aim was to investigate the predictive value of neutrophil gelatinase-associated lipocalin (NGAL) and kidney injury molecule 1 (KIM-1) in the early diagnosis of contrast-induced nephropathy $(\mathrm{CIN})$ in patients after percutaneous coronary intervention $(\mathrm{PCl})$.
\end{abstract}

Method: We measured serum NGAL and KIM-1 using commercially available kits before and after 24 and 48 hours following $\mathrm{PCl}$ in 61 patients. We also measured serum creatinine ( $\mathrm{sCr}$ ) at the baseline and after 24, 48 and 72 hours to detect patients who developed CIN.

Results: The non-CIN group comprised 32 patients and CIN group 29 patients. Mean age was $68,1+-10,5$ years $(68,1+-11,2$ in the CIN group, 68,4+-10,6 in non-CIN group). We observed significantly higher levels of NGAL marker just after the procedure in CIN group than in non-CIN group $(92,1+-74,8$ vs 50,2+-26,9 respectively, $p=0,00)$. Similarly, 24 and 48 hours after the procedure, the level of NGAL was significantly higher in CIN group (92,1+-74,8 vs 50,2+-26,9 $, p=0,00$ and $99,2+-93,2$ vs $46,3+-23,9, p=0,00$ respectively). Regarding $\mathrm{KIM}-1$, higher levels were observed only 24 hours after PCl: mean level of KIM-1 was 525,5+-1120,1 in the CIN group and $162,0+-335,9$ in non-CIN group $(p=0,02)$. Data collected at baseline and 48 hours after contrast injection are not statistically significant, however, mean levels of KIM-1 are higher in CIN group than in non-CIN group ( 477,1+-1074,2 vs $150,0+-291,8,0$ hour, CIN vs non CIN and 508,6+1139,3 vs $165,1+-305,2$.

Conclusion: NGAL is a useful early marker for nephrotoxicity and predictor of $\mathrm{CIN}$ after PCI and in this setting seems to have superiority over KIM-1.

No conflict of interest 
ANGINA PECTORIS, UNSTABLE ANGINA, ACS

\title{
ACS IN A PATIENT WITH A SINGLE CORONARY ARTERY AND THE PRESENCE OF A DOUBLE LAD, AN EXTREMELY RARE CASE OF CONGENITAL ANOMALY OF CORONARIES
}

\author{
C. Graidis ${ }^{1}$, D. Dimitriadis ${ }^{1}$, A. Mavrogianni ${ }^{2}$, G. Dimitriadis ${ }^{2}$, V. Karasavvidis ${ }^{1}$, T. Bitsis ${ }^{1}$, \\ A. Tsiakou', I. Bostanitis ${ }^{2}$, I. Zarifis ${ }^{2}$ \\ ${ }^{1}$ Euromedica Kyanous Stavros General Hospital, Interventional Cardiology Department, \\ Thessaloniki, Greece \\ 2Papanikolaou General Hospital, Interventional Cardiology Department, Thessaloniki, Greece
}

Aims: Congenital coronary anomalies are uncommon with an incidence 35 ranging from $0.17 \%$ in autopsy cases to $1.2 \%$ in angiographically evaluated cases. The recent development of ECGgated multi-detector row computed tomography (MDCT) coronary angiography allows accurate and noninvasive depiction of coronary artery anomalies. We present a unique case of a patient that was admitted to our department with acute coronary syndrome and his coronary angiography revealed an extremely rare congenital coronary artery anomaly.

Method: In this case the patient underwent coronary angiography that revealed the coexistence of a two distinct coronary artery anomalies, the presence of a single coronary artery (SCA type $R / 3 / P$ ) together with the existence of a double left anterior descending artery (double LAD type IV). Subsequently a 64 multi slice CT coronary angiography with injection of $80 \mathrm{ml}$ nonionic contrast medium. Retrospective gating technique was used to synchronize data reconstruction with the ECG signal. Maximum intensity projection, multi-planar reformatted, and volume rendering images were derived from axial scans.

Results: The results of this study support the use MDCT coronary angiography as a safe and effective noninvasive imaging modality for defining CAAs in an appropriate clinical setting, providing detailed three-dimensional anatomic information that may be difficult to obtain with invasive angiography. 


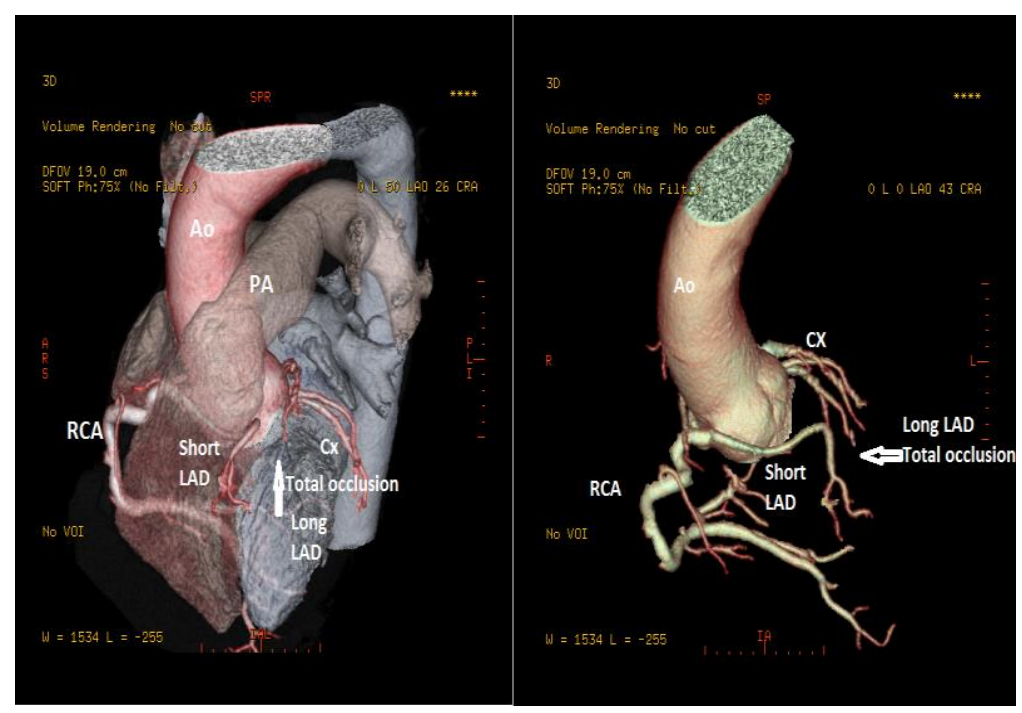

Conclusion: It's of great importance the diagnostic approach to cases like that, in order to properly design the conservative, surgical or percutaneus treatment. To our knowledge the above coronary artery variation has not described in the literature yet.

No conflict of interest 
ANGINA PECTORIS, UNSTABLE ANGINA, ACS

\title{
ASSOCIATION OF SERUM GAMMA-GLUTAMYL TRANSFERASE AND PREMATURE CORONARY ARTERY DISEASE
}

\author{
A. Shafiee ${ }^{1}$, M. Sheikh ${ }^{2}$, A. Amirzadegan 3 , M. Salarifar ${ }^{1}$ \\ ${ }^{1}$ Tehran Heart Center- Tehran University of Medical Sciences, Tehran, Iran \\ ${ }^{2}$ Tehran Heart Center- Tehran University of Medical Sciences, Cardiology, Tehran, Iran \\ ${ }^{3}$ Tehran Heart Center- Tehran University of Medical Sciences, Interventional cardiology, Tehran, \\ Iran
}

Aims: Serum Gamma-Glutamyl Transferase (GGT) has been introduced as a predictive factor for cardiovascular disease. In this study, we investigated the association of serum GGT and premature coronary artery disease (CAD) in the candidates for coronary angiography.

Methods: In this cross sectional study, we enrolled male subjects aged $\leq 45$ years and female subjects $\leq 55$ years who were candidate for elective coronary angiography due to typical chest pain or positive non-invasive test. Baseline characteristics were recorded for all the participants and serum levels of blood sugar, lipid profile and GGT were measured. Patients were then divided into $C A D$ and non-CAD groups based on angiography for further comparisons.

Results: From a total of 367 patients (age=45.1 \pm 6.1 years, male=161 [43.9\%]), $176(47.9 \%)$ patients had premature CAD. High level of GGT was significantly associated with the extent of the CAD $(P<0.001)$. Ten units increase in GGT could strongly predict the presence of premature coronary artery disease (OR: 13.34, 95\% Cl: 7.19-24.78; P-value<0.001) after adjustment for confounders. The area under the receiver operating characteristic curve for GGT was $80.9 \%$ (ranged 76.5-85.3) and the sensitivity and specificity of GGT for predicting premature CAD at a cut-point of $22.5 \mathrm{IU} / \mathrm{I}$ was $80.1 \%$ and $70.2 \%$, respectively. Every 10 unit increase in GGT was predictive for one level increase the vessel score (OR: 1.63, 95\% Cl: 1.46-1.82; P-value<0.001).

Conclusion: We observed that GGT levels in patients with typical chest pain or positive noninvasive tests could predict the presence and extent of premature CAD.

No conflict of interest 
ATHEROSCLEROSIS, LIPIDS, LIPOPROTEINS

\title{
THE -514 C/T HEPATIC LIPASE PROMOTOR REGION POLYMORPHISM AND PREMATURE CORONARY ARTERY DISEASE IN AN IRANIAN POPULATION
}

\author{
H. Goodarzynejad' ${ }^{1}$ M. Boroumand ${ }^{2}$, M. Behmanesh ${ }^{3}$, S. Ziaee ${ }^{4}$, A. Jalali', L. pourgholit \\ ${ }^{1}$ Tehran Heart Center, Cardiac Research, Tehran, Iran \\ ${ }^{2}$ Tehran Heart Center, Tehran, Iran \\ ${ }^{3}$ Tarbiat Modares University, Human Genetics, Tehran, Iran \\ ${ }^{4}$ Tehran Heart Center, Surgical Pathology and Laboratory Medicine, Tehran, Iran
}

Aims: Hepatic lipase (HL) plays a crucial role in lipid metabolism but there is debate about whether $\mathrm{HL}$ acts in a more pro- or more anti-atherogenic fashion. The aim of this study was to examine the relationship between the $-514 \mathrm{C} / \mathrm{T}$ polymorphism within $\mathrm{HL}$ gene (LIPC) and the risk of angiographically determined premature coronary artery disease (CAD).

Method: An age cut-off of 55 years for women and 45 years for men was used to define premature CAD. Four-hundred and seventy-one patients with newly diagnosed angiographically documented PCAD ( $\geq 50 \%$ luminal stenosis of any coronary vessel) as case group compared to 503 controls (subjects with no luminal stenosis at coronary arteries). A real-time polymerase chain reaction (PCR) and High Resolution Melting (HRM) analysis was used to distinguish between genotypes.

Results: There was no statistically significant difference in the distribution of $-514 \mathrm{C} / \mathrm{T}$ genotypes between two groups in whole population. After stratification by sex, the examined polymorphism was found to be associated with the presence of CAD in women $(p=0.029)$ but not in men $(p=$ 0.651 ) in univariate analysis. After applying a multiple logistic regression model with adjusting for other covariates, the examined polymorphism was not found to be associated with the presence of CAD in total population or in women and men separately.

Conclusion: Our findings suggest that there is no relationship between LIPC $-514 \mathrm{C} / \mathrm{T}$ polymorphism and the risk of premature CAD among a relatively large number of Iranian patients undergoing coronary angiography.

No conflict of interest 
257

ATHEROSCLEROSIS, LIPIDS, LIPOPROTEINS

\title{
THE SCAVENGER RECEPTOR CLASS B TYPE 1 (SCARB1) RS5888 POLYMORPHISM AND PREMATURE CORONARY ARTERY DISEASE IN AN IRANIAN POPULATION
}

\author{
H. Goodarzynejad ${ }^{1}$, M. Boroumand ${ }^{2}$, M. Behmanesh ${ }^{3}$, S. Ziaee ${ }^{4}$, A. Jalali ${ }^{1}$ \\ ${ }^{1}$ Tehran Heart Center, Cardiac Research, Tehran, Iran \\ ${ }^{2}$ Tehran Heart Center, Tehran, Iran \\ ${ }^{3}$ Tarbiat Modares University, Human Genetics, Tehran, Iran \\ ${ }^{4}$ Tehran Heart Center, Surgical Pathology and Laboratory Medicine, Tehran, Iran
}

\section{Aims:}

Several single nucleotide polymorphisms (SNPs) in lipid transport genes have been shown to be associated with premature coronary artery disease (PCAD). The scavenger receptor $\mathrm{BI}$ (SCARB1) is a key component of the reverse cholesterol transport and lipid metabolism. We aimed to examine the relationship between the rs5888 SNP within SCARB1and the risk of angiographically determined PCAD.

\section{Method:}

We used an age cut-off of 55 years for women and 45 years for men to define PCAD. Fivehundred and five patients with newly diagnosed angiographically documented PCAD ( $\geq 50 \%$ luminal stenosis of any coronary vessel) as case group compared with 546 controls (subjects with no luminal stenosis at coronary arteries). The severity of CAD was determined by vessel score as well as Gensini score. A real-time polymerase chain reaction (PCR) and High Resolution Melting (HRM) analysis was used to distinguish between genotypes.

\section{Results:}

TT genotype as compared to CC genotype was associated with increased odds of PCAD in total population (adjusted $\mathrm{OR}=1.7,95 \% \mathrm{Cl}=1.1$ to $2.6 ; \mathrm{p}=0.014$ ), and in women (adjusted $\mathrm{OR}=2.1$, $95 \% \mathrm{Cl}=1.2$ to $4.0 ; \mathrm{p}=0.015$ ), but not in men (adjusted $\mathrm{OR}=1.3,95 \% \mathrm{Cl}=0.7$ to $2.5 ; \mathrm{p}=0.350$ ). There was also no significant association between the examined polymorphism and the severity of $C A D$ in whole or in men or women subgroups.

\section{Conclusion:}

Our findings suggest that the SNP ( $r 5588$ ) within SCARB1 is independently associated with PCAD in a sex-dependent manner.

No conflict of interest 


\title{
ATHEROSCLEROSIS, LIPIDS, LIPOPROTEINS \\ THROMBOLYSIS THERAPY AFTER A FIRST ACUTE ISCHEMIC STROKE: GENDER DIFFERENCES IN LONG TIME SURVIVAL AND DISABILITY. EBRICTUS STUDY
}

\author{
J.L. Clua-espuny ${ }^{1}$, R. Ripolles-Vicente ${ }^{1}$, G. Procida-Cervera', M.L. Queralt-Tomas ${ }^{1}$, \\ M.A. Gonzalez-Henares ${ }^{1}$, A. Panisello-Tafalla ${ }^{1}$, T. Forcadell-Arenas ${ }^{1}$, F. Ferreira-Caro ${ }^{1}$, V.F. Gil- \\ Guillen $^{2}$ \\ ${ }^{1}$ Institut Catala de la Salut, Primary Care, Tortosa, Spain \\ ${ }^{2}$ Elx University, Family Medicine. Primary Care Dpt, Elx, Spain
}

Aims: We explored whether sex might modify the effect of thrombolysis on survival and functional outcomes in patients with acute ischemic stroke ${ }^{12}$ beyond the usual of 6 -month after stroke and comparing with group without thrombolytic treatment.

Method: Prospective cohort study of a population-based cohort of 1339 consecutive incident strokes (614 in women) from 01/04/2006 to 31/12/2014. Statistical approaches for analyzing survival outcomes.

Results: The death rates were lower ( $p$ 0.003) in thrombolysis therapy group with IR 0.57 (CI95\% $0.39-0.83) .113(8.8 \%)$ patients (53 women) received thrombolysis. They were significantly ( $\mathrm{p}$ $<0.001)$ younger $(69.2 \pm 12.7$ vs $73.9 \pm 12.5$ years $)$, had $(p<0.001)$ higher NIHSS score $(12.7 \pm 6.3$ vs $7.3 \pm 7.0)$, spent $(p<0.001)$ more hospital days $(10.4 \pm 8.3$ vs $8.3 \pm 7.9)$, higher $(p 0.023)$ average Barthel score at discharge $(85.5 \pm 24.4$ vs $79.2 \pm 28.6)$. The male/female IR showed a significant decrease ( $p$ 0.01) in the incidence of mortality in women and better Barthel score. The thrombolysis improved the survival in overall group ( $p 0.028$ ) and in women vs men ( $p$ 0.023); and in women with thrombolysis vs not $(p<0.001)$; but not in men with thrombolysis vs not ( $p$ 0.743 ). The protective factors were: thrombolysis (CI95\% 0.37-0.80 p 0.002); Barthel $\geq 60$ score (CI95\% 0.81-0.94 p 0.002), and the cardiovascular secondary prevention one year $(0.13$ IC95\% 0.06-0.28) after stroke.

Conclusion: The stroke death rates were lower in women after thrombolysis treatment. The overall benefit on survival of the patients treated with thrombolysis might be explained by the beneficial effect of the thrombolysis on the women.

No conflict of interest 


\title{
ATHEROSCLEROSIS, LIPIDS, LIPOPROTEINS
}

\section{PREVALENCE OF HYPERTRIGLYCERIDEMIA AND CORRELATION BETWEEN TRIGLYCERIDE LEVELS AND GLYCATED HEMOGLOBIN IN THE RUSSIAN POPULATION (PROMETHEUS STUDY)}

\author{
N. Gurina ${ }^{1}, Y$. Karpov $^{2}$ \\ ${ }^{1}$ AstraZeneca, Medical, Moscow, Russia \\ ${ }^{2}$ Russian Cardiology Research and Production Complex, Problems of atherosclerosis, Moscow, \\ Russia
}

Aims: The primary objective of the study was to estimate the percentage of patients with hypertriglyceridemia in Russia. One of the secondary targets was to assess the correlation between glycated hemoglobin $\left(\mathrm{HbA}_{1 c}\right)$ and triglyceride level.

Method: This is a cross-sectional retrospective study of outpatient individuals. Men and women aged 18 years and older tested in the Russian centres of INVITRO Laboratory in the period of 3 years (2011-2013) were eligible for the analysis. The full dataset contains lipoprotein analysis from 357,072 individual records. Individuals with reported levels of $\mathrm{HbA}_{1 \mathrm{c}}$ were identified as a nested dataset $(n=54,602)$. Correlations were assessed by one-way linear regression.

Results: In the full dataset study population, $29.2 \%(95 \% \mathrm{Cl}, 29.1 \%-29.4 \%)$ of individuals had hypertriglyceridemia (serum triglyceride $\geq 1.7 \mathrm{mmol} / \mathrm{L}$ ). Hypertriglyceridemia was more prevalent in the nested sample at $36.5 \%(95 \% \mathrm{Cl}, 36.1 \%-36.9 \%, \mathrm{p}<0.0001)$. The mean $\mathrm{HbA} 1 \mathrm{c}$ was $6.1 \%$. The prevalence of high $\mathrm{HbA} 1 \mathrm{c}$ level $(\geq 6.5 \%)$ was $11.0 \%(95 \% \mathrm{Cl}, 10.7 \%-11.2 \%)$. The log-linear model predicts $9.3 \%$ increase in triglyceride level with each 1-unit increase in $\mathrm{HbA} 1 \mathrm{c}$ level $(p<0.0001)$. Risk of hypertriglyceridemia was 1.7 times greater when high $\mathrm{HbA1c}$ was already present, and vice versa, risk of $\mathrm{HbA} 1 \mathrm{c} \geq 6.5 \%$ was 2 times higher in individuals with hypertriglyceridemia.

Conclusion: In the Russian population, $29.2 \%$ had hypertriglyceridemia in the full dataset, whereas the prevalence of hypertriglyceridemia was significantly higher in the nested sample. There is a linear association between high $\mathrm{HbA} 1 \mathrm{c}$ and high level of triglyceride that could be a result of higher prevalence of hypertriglyceridemia in patients with diabetes mellitus.

Conflict of Interest Disclosure Statement:

This project was funded by AstraZeneca. One of the co-author Natalia Gurina is an AstraZeneca employee. 


\title{
ATHEROSCLEROSIS, LIPIDS, LIPOPROTEINS
}

\section{PHYTANIC ACID AND PLASMALOGEN-C16:0 AS A NEW MARKERS OF CORONARY ARTERY DISEASE IN TUNISIAN PATIENTS}

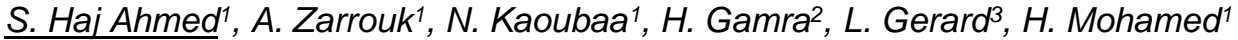 \\ ${ }^{1}$ Faculty of Medicine, Biochemistry department- Research Laboratory LR12ES05 LR- \\ NAFS 'Nutrition - Functional Food \& Vascular Health', Monastir, Tunisia \\ ${ }^{2} \mathrm{CHU}$ Fattouma Bourgiba Monastir, Cardiologiy Service CHU Fattouma Bourgiba Monastir- \\ Tunisia, MONASTIR, Tunisia \\ ${ }^{3}$ University of Bourgogne / INSERM- Dijon- France, Biochemistry of Peroxisome Team- \\ Inflammation and Metabolism Lipid EA 7270 / University of Bourgogne / INSERM- Dijon- France, \\ Dijon, France
}

\begin{abstract}
Aims: Modifications of peroxisomal metabolism are suspected to contribute to the development of coronary artery disease (CAD). Thus, we intended to determine possible correlations between peroxisomal metabolism markers and vascular severity, estimated with Gensini score.

Method: 150 patients were clinically examined by coronary angiography and classified as CAD and non-CAD group, and 120 controls are matched by age and sex. Several markers of peroxisomal metabolism including phytanic acid and plasmalogen-C16:0 were simultaneously analyzed by GC/MS-SIM. Markers of lipid peroxidation (malondialdehyde (MDA) and conjugated dienes (CD)) were analysed in erythrocytes.
\end{abstract}

Results: Plasmalogen-C16:0 concentration was almost twice as high in CAD and non-CAD groups than controls $(28.02 \pm 7.81$ and $27.21 \pm 8.38 \mu \mathrm{M}$ vs $15.14 \pm 3.42 \mu \mathrm{M}$, respectively). It was negatively correlated with the severity of CAD. Phytanic acid concentration, was 7 times higher in non-CAD and CAD groups than controls $(81.04 \pm 10.22 \mu \mathrm{M}$ and $83.70 \pm 12.09 \mu \mathrm{M}$ vs $12.92 \pm 3.74$ $\mu \mathrm{M}$, respectively). Analysis of the ROC curve showed that phytanic acid might be an important predictor of CAD. These accumulations was supposed to reveal peroxisomal dysfunctions. We noticed also, a significant increase of MDA and CD levels in CAD group. A positive correlation of $C D$ with the severity of CAD was revealed $(r=0.291, P<0.05)$. In addition, a significant negative correlation between the level of MDA and plasmalogen-C16:0 was observed in CAD group.

Conclusion: The accumulation of phytanic acid and plasmalogen-C16:0 reflect alteration of peroxysomal metabolism and could constitute suitable peripheric biomarkers of CAD. These parameters with lipid peroxydation markers are associated with vascular severity.

No conflict of interest 
261

\title{
ATHEROSCLEROSIS, LIPIDS, LIPOPROTEINS
}

\section{RELATION OF LPAI AND PREBETA-1 HDL LEVELS IN PATIENTS WITH ANGIOGRAPHICALLY PROVEN CORONARY ARTERY DISEASE}

\author{
A. Kuchta ${ }^{1}$, A. Strzelecki ${ }^{2}$, A. Ćwiklińska ${ }^{1}$, B. Kortas-Stempak ${ }^{1}$, M. Gruchała ${ }^{3}$, Z. Zdrojewski ${ }^{2}$, \\ K. Dąbkowski ${ }^{1}$, A. Gliwińska ${ }^{1}$, M. Jankowski ${ }^{1}$ \\ ${ }^{1}$ Medical University of Gdańsk, Department of Clinical Chemistry, Gdańsk, Poland \\ ${ }^{2}$ Medical University of Gdańsk, Chair and Clinic of Internal Medicine- \\ Connective Tissue Diseases and Geriatrics, Gdańsk, Poland \\ ${ }^{3}$ Medical University of Gdańsk, First Chair \& Clinic of Cardiology, Gdańsk, Poland
}

\begin{abstract}
Aims: High density lipoproteins (HDL) are composed of two metabolically distinct fractions, one containing ApoAl but not ApoAll (LPAI) and that containing both ApoAl and ApoAll (LPAl/All). In vitro studies have shown that only LPAI promotes cholesterol efflux, suggesting that it is fraction responsible for antiatherogenic properties of HDL. LPAI fraction is itself heterogeneous and preß1-HDL is a minor subfraction of LPAI and the initial acceptor of cellular cholesterol in the process of reverse cholesterol transport. However previous studies have shown increase in preß1-HDL concentration in coronary heart disease. The aim of the study was to estimate plasma LPAI and preß1-HDL concentrations and to evaluate the relationship between these two parameters in patients with angiographically proven coronary artery disease (CAD).
\end{abstract}

Method: The study included 82 patients with angiographically documented CAD (CAD+) and 45 patients with negative results of coronary angiography (CAD-).

Results: Compared to the control group CAD+ patients were characterized by reduced level of LPAl ( $45 \pm 23$ vs $54 \pm 24 \mathrm{mg} / \mathrm{dL} ; \mathrm{P}=0.04)$ but increased preß1-HDL level $(4.2 \pm 1.5 \mathrm{vs} 3.6 \pm 1.1 \mathrm{mg} / \mathrm{dL}$ $\mathrm{P}=0.02)$. There was no correlation between $\mathrm{LPAl}$ and preß1-HDL concentration. The ratio preß1$\mathrm{HDL} / \mathrm{LPAl}$ was significantly higher in CAD+ compared to the CAD- group $(0.12 \pm 0.074$ vs $0.08 \pm 0.05 ; P=0.004)$ and positively correlated with the severity of $C A D$, evaluated by the Gensini Score $(R=0.49, P<0.001)$.

Conclusion: The results support earlier reports of a reduced LPAI level and elevated concentration of preß1-HDL in CAD+ patients and suggest that the preß1-HDL/LPAI ratio may be considered as additional marker of coronary artery disease.

No conflict of interest 
262

ATHEROSCLEROSIS, LIPIDS, LIPOPROTEINS

\title{
HDL ENHANCES THE EFFICIENCY OF LIPOPROTEIN LIPASE (LPL)-MEDIATED VLDL LIPOLYSIS IN NON-DIALYSED PATIENTS WITH CHRONIC KIDNEY DISEASE
}

\author{
A. Ćwiklińska ${ }^{1}$, M. Cackowska ${ }^{2}$, E. Wieczorek ${ }^{1}$ A. Kuchta ${ }^{1}$, E. Król', B. Kortas-Stempak ${ }^{1}$, \\ A. Gliwińska ${ }^{1}$, K. Dąbkowski ${ }^{1}$, M. Jankowski ${ }^{1}$ \\ ${ }^{1}$ Medical University of Gdańsk, Department of Clinical Chemistry, Gdańsk, Poland \\ ${ }^{2}$ Medical University of Gdańsk, Chair \& Clinic of Nephrology- \\ Transplantology and Internal Diseases, Gdańsk, Poland
}

\begin{abstract}
Aims: The aim of this study was to assess the effect of high density lipopoprotein (HDL) on very low density lipoprotein (VLDL) lipolysis in non-dialysed chronic kidney disease (CKD) patients.

Method: Adult non-dialysis CKD patients (age $66 \pm 12 ; n=15$ ) treated at the Nephrology Clinic of Medical University of Gdansk were recruited to the study. VLDL was isolated from blood serum by ultracentrifugation. $\alpha-H D L$ was isolated by combined precipitation-ultracentrifugation procedure. VLDL was incubated with LPL (1 hour, $37^{\circ} \mathrm{C}$, VLDL-TG:LPL 90:0.48mg/dl, $2 \%$ albumin), in the absence or presence of HDL (VLDL-cholesterol:HDL-cholesterol 1:1). After incubation VLDL was separated by immunoprecipitation and the percentage of hydrolysed TG was calculated.
\end{abstract}

Results: The percentage of TG hydrolysed during reaction was $80 \%$ (55-94) and $85 \%$ (74-92) (mean, min-max), for $\alpha-H D L$ absence and presence, respectively $(p<0.007)$. For samples with $\mathrm{TG}<60 \mathrm{mg} / \mathrm{dl}$ there was no effect of $\alpha-\mathrm{HDL}$ on TG lipolysis. For samples with $\mathrm{TG}>60 \mathrm{mg} / \mathrm{dl}$ the impact of $\alpha-\mathrm{HDL}$ on lipolysis increased with the increase in TG concentration. The greatest impact of $\alpha-H D L$ was for samples with TG concentration above $100 \mathrm{mg} / \mathrm{dl}$, where the $\alpha-\mathrm{HDL}$ enhances the efficiency of lipolysis by $20 \%$ (Figure). 


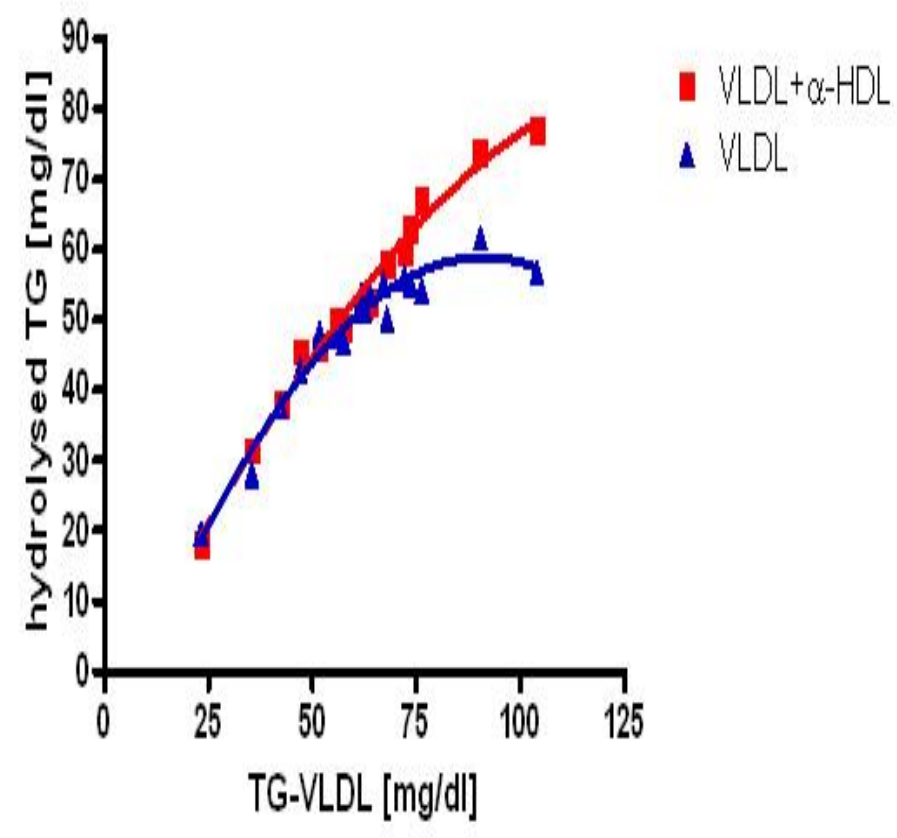

Conclusion: $\alpha-H D L$ enhances the efficiency of LPL-mediated VLDL lipolysis in patients with CKD, in samples with higher TG concentration. We suspect that this activity is most likely related to HDL activity as acceptors of surface material released from VLDL during lipolysis.

No conflict of interest 
263

\title{
ATHEROSCLEROSIS, LIPIDS, LIPOPROTEINS \\ LYSOPHOSPHATIDYLCHOLINE ACTIVATES THE AKT PATHWAY IN HUMAN AORTIC VALVE CELLS TO UP-REGULATES THE PRODUCTION OF BIGLYCAN AND COLLAGEN I
}

\author{
X. Meng, H. Cheng, Q. Yao, R. Song, L. Ao, D. Fullerton \\ University of Colorado Denver, Surgery, Aurora, USA
}

\begin{abstract}
Aims: Overproduction of extracellular matrix (ECM) protein by aortic valve interstitial cells (AVICs) plays an important role in valvular sclerosis (thickening) associated with the early pathobiology of calcific aortic valve disease (CAVD). Accumulation of oxidized low-density lipoprotein (oxLDL) is observed in diseased aortic valves and may have a mechanistic role in CAVD progression. We have found that oxLDL up-regulates the production of biglycan in human AVICs. This study determines the mechanism by which oxLDL modulates ECM protein production.
\end{abstract}

Method: AVICs were isolated from normal human aortic valves. Cells were treated with oxLDL $(40 \mu \mathrm{g} / \mathrm{ml})$ or lysophosphatidylcholine (LysoPC, $40 \mu \mathrm{M} / \mathrm{L})$, a component of oxLDL.

Immunoblotting was applied to analyze ECM proteins (collagens I, III, IV and biglycan) in cell lysate.

Results: OxLDL up-regulated the production of biglycan and collagens I at $48 \mathrm{~h}$, while LysoPC increased the production of these two ECM proteins at $24 \mathrm{~h}$. The up-regulation of ECM proteins by LysoPC was preceded by the phosphorylation of Akt and ERK1/2, but not p38 MAPK. Inhibition of Akt markedly reduced the effect of LysoPC on ECM protein production while inhibition of ERK1/2 resulted in a moderate reduction.

Conclusion: LysoPC up-regulates the production of biglycan and collagen I in human AVICs and may mediate the effect of oxLDL on ECM protein production. The Akt pathway appears to be critical in mediating the effect of LysoPC. OxLDL accumulation and generation of LysoPC in the aortic valve may contribute to the mechanism of valvular sclerosis associated with the progression of CAVD.

No conflict of interest 
264

\title{
ATHEROSCLEROSIS, LIPIDS, LIPOPROTEINS
}

\section{PATHOGENESIS OF EARLY AND INTERMEDIATE LESIONS OF HUMAN CORONARY ATHEROSCLEROSIS: ACCUMULATION OF PLASMA-DERIVED LIPIDS AND DISPERSION OF SMOOTH MUSCLE CELLS}

\author{
K. Nakagawa ${ }^{1}$, H. Fujiii', Y. Nakashima ${ }^{2}$ \\ ${ }^{1}$ Kyushu University, Pathophysiological and Experimental Pathology, Fukuoka, Japan \\ 2Japanese Red Cross Fukuoka Hospital, Division of Pathology, Fukuoka, Japan
}

\begin{abstract}
Aims: Proliferation of smooth muscle cells (SMCs) and deposition of lipids by death of macrophage foam cells are key events in atherosclerosis in animal models. However, different processes occur in human atherosclerosis. The purpose of this study is to reveal sequential processes of earlier stages of human coronary atherosclerosis, focusing on extracellular lipids and SMCs.
\end{abstract}

Method: Frozen sections of the right coronary artery were obtained from 43 Japanese autopsied subjects who died in 15-49-years-old with no or earlier atherosclerotic lesions. Lesions were classified into 4 categories according to the grade of atherosclerosis, and immunohistochemically analysed.

Results: Diffuse intimal thickening was a physiological intimal thickening with plenty of SMCs. Initial lesions and fatty streaks exhibited mild deposition of extracellular lipids and accumulation of macrophage foam cells in the SMC-rich intima, respectively. Intermediate lesions consisted of lipid pools with abundant extracellular lipids in the thickened intima. No necrosis or lytic changes were observed in lipid pools, and extracellular lipids colocalized with ApoB and fibrinogen, suggesting that the penetration of plasma lipoproteins played important roles in lipid pool formation. The density of intimal SMCs was significantly decreased in fatty streaks and intermediate lesions, but total SMC counts showed no significant change. Moreover, there were little or no positive cells in MIB-1, BAX, LC3B and TUNEL staining, suggesting that SMCs were dispersed in intima without undergoing excessive proliferation or death.

Conclusion: Early and intermediate lesions of human coronary atherosclerosis develop through infiltration and accumulation of plasma-derived lipids and dispersion of preexisting intimal SMCs.

No conflict of interest 
ATHEROSCLEROSIS, LIPIDS, LIPOPROTEINS

\title{
INTER-ARM BLOOD PRESSURE DIFFERENCES ASSOCIATED WITH CORONARY ATHEROSCLEROSIS ANALYSED BY CORONARY ANGIOGRAPHY
}

\author{
S.J. Park, J.W. Son, S.M. Park, H.H. Choi, K.S. Hong \\ Hallym Univ., Cardiology, ChunChoen, Korea
}

\begin{abstract}
Aims: Inter-arm blood pressure differences (IABPD) is related to atherosclerosis including peripheral and cerebral vascular disease. There is a controversy about the relationship between IABPD and coronary artery disease.
\end{abstract}

Method: We measured blood pressure in both arms simultaneously in 412 patients (240 male, age $66.6 \pm 11.2$ years) underwent their first coronary angiography. The extent of coronary atherosclerosis was assessed using the Gensini score based on the quantitative coronary angiography results. The coronary artery tree was divided into 14 segments and each of these segments was scored from 1 to 32 depending on the most severe stenosis. The Gensini score was presented as the sum of the scores in all segments.

Results: Mean and median Gensini scores of the enrolled patients were $34.9 \pm 32.0$ and 25 . IABPDs in systole (SIABPD) and diastole (DIABPD) revealed positive correlation with the Gensini score. The ratios of SIABPD and DIABPD to the mean blood pressure were also correlated with the Gensini score positively (Table). The mean SIABPD and DIABPD were more greater in the high Gensini group ( $\geq 25$ ) than low Gensini group (mean SIABPDs: $5.6 \pm 6.6$ vs $4.1 \pm 4.8, p=0.015$; mean DIABPDs: $4.0 \pm 6.1$ vs $3.0 \pm 3.2, p=0.043$, respectively). The SIABPD might predict the higher Gensini score (Area of under the curve: $0.62,95 \%$ confidence interval: $0.57-0.67, p<0.001$ ).

\section{Correlation coefficient $(r) \quad p$ value}

SIABPD

DIABPD

mean IABPD

SIABPD/mean blood pressure

DIABPD/mean blood pressure
0.099

0.045

0.097

0.049
0.207

0.109

0.101

0.041

0.108

0.029

Conclusion: SIABPD and DIABPD were related to the severity of coronary artery disease as shown in peripheral and cerebral artery disease. It might be needed to detect IAPBDs, and to manage coronary atherosclerosis for prevention of cardiac events.

No conflict of interest 
ATHEROSCLEROSIS, LIPIDS, LIPOPROTEINS

\title{
AN INCREASE OF MAXIMAL INTIMA-MEDIA THICKNESS IN BILATERAL CAROTID ARTERY IS ASSOCIATED WITH CORONARY STENOSIS IN JAPANESE DIABETIC PATIENT
}

\author{
H. Shimizu \\ Maebashi Hirosegawa Clinic, Diabetes Center, Maebashi, Japan
}

\begin{abstract}
Aims: Screening and treatment of asymptomatic coronary artery disease (CAD) is important to prevent cardiac event in diabetic patients. Maximal intima-media thickness (IMT) of carotid artery is a non-invasive marker to assess the development of atherosclerosis. An association of maximal IMT to coronary artery stenosis was evaluated in Japanese type 2 diabetic (T2DM) outpatients without any typical symptoms or history of CAD.

Method: IMT from common carotid artery to internal carotid artery was measured by ultrasonography and maximal IMT was determined. In the patients with an increase of maximal IMT (>1.5 mm) in bilateral carotid arteries, the existence of coronary artery stenosis was evaluated by multiple detector-row computed tomography (MDCT). In the patients with coronary artery stenosis (CAS) suspected by MDCT, the existence of CAD was evaluated by coronary angiography, and the necessity of percutaneous coronary intervention $(\mathrm{PCI})$ therapy was determined.
\end{abstract}

Results: An increase of maximal IMT was found in bilateral carotid arteries of 32 patients. Twenty two patients $(68.8 \%)$ showed CAS by MDCT, and 12 patients $(37.5 \%)$ needed $\mathrm{PCl}$. There was no difference in glycemic control and serum LDL-C concentration between the patients with and without CAS. Treatment with statin did not affect the incidence of CAS $(75.0 \%)$ and PCI (25.0\%). However, the patients, in whom serum LDL-C concentration was lowered below 70 $\mathrm{mg} / \mathrm{dl}$ by statin treatment, did not have any CAS at all.

Conclusion: An increase of maximal IMT in bilateral carotid arteries is associated with CAS, and indicates the necessity of further screeing of CAS and treatment.

No conflict of interest 


\title{
ATHEROSCLEROSIS, LIPIDS, LIPOPROTEINS
}

\section{THE INFLUENCE OF METABOLIC SYNDROME ON SMALL DENSE LDL AND SUBCLINICAL ATHEROSCLEROSIS IN NON-ELDERLY JAPANESE DIABETIC SUBJECTS}

\author{
G. Yoshino \\ Shin-Suma General Hospital, Center for Diabetes, Kobe, Japan
}

Aims: The present study was conducted to examine the influence of an association of metabolic syndrome (MS) on small dense LDL-cholesterol (sLDL-C) and subclinical atherosclerosis in nonelderly diabetic subjects.

Method: A total of 130 subjects including 88 male and 42 female, ranging from 30 to 65 years of age, were recruited. The subjects who had hepatic, renal or thyroid diseases were excluded. Fibrate and statin users were also excluded. They are divided into 4 groups, ie: healthy nondiabetic and non-MS group、 non-MS diabetic group, non-diabetic MS group、 and diabetic MS group. Presence of MS was diagnosed according to the Japanese Guidelines for the Definition of Metabolic Syndrome. After informed consent was given, blood sampling was done after overnight fast. Plasma lipids were measured using an auto-analyzer. sLDL-C was measured employing a direct assay method established by Hirano et al. Subclinical atherosclerosis was estimated as plaque score using echogram at carotid artery.

Results: The subjects with MS had significantly higher fasting blood glucose, LDL-C, triglyceride, and SLDL-C than non-MS subjects. Diabetic MS group had significantly higher hs-CRP and SLDL$C$ than non-MS diabetic group. The diabetic MS group had the highest hs-CRP, sLDL-C and plaque score of the carotid artery among the 4 groups. Multiple regression analysis revealed that the most powerful determinant of plaque score of the carotid artery was age, followed by gender, presence of MS, type 2 diabetes and SLDL-C.

Conclusion: An association of MS increases hs-CRP, sLDL-C and subclinical atherosclerosis in non-elderly type 2 diabetic Japanese subjects.

No conflict of interest 
371

\title{
ATHEROSCLEROSIS, LIPIDS, LIPOPROTEINS \\ INCIDENCE OF INSUFFICIENT PLASMA POLYUNSATURATED FATTY ACID CONCENTRATION IN ISCHEMIC HEART DISEASE PATIENTS WITH OPTIMAL PLASMA LDL CONCENTRATION
}

\author{
S. Yoshida, H. Adachi \\ Gunma Prefectural Cardiovascular Center, Crdiology, Maebashi- Gunma, Japan
}

Aims: Background and Purpose: Although effects of dietary fat quality on cardiovascular risk is well documented, and polyunsaturated fatty acid (PUFA) is known as one of the important factors to reduce the ischemic heart disease (IHD), its plasma concentration in IHD patient is still unclear. Hereby, we investigated the incidence of insufficient PUFA concentration in IHD patients with optimal LDL concentration.

Methods: In 48 IHD patients after successful percutaneous coronary intervention (PCI), plasma eicosapentaenoic acid (EPA), docosahexaenoic acid (DHA), arachidonic acid (AA) and other lipid profiles were measured at 9 months after $\mathrm{PCl}$.

Result: Strong statin was taken by all patients. At 9 months after PCI, LDL cholesterol level was controlled below $100 \mathrm{mg} / \mathrm{dl}$ in $85.4 \%$ patients although only $31.3 \%$ was below $100 \mathrm{mg} / \mathrm{dL}$ before $\mathrm{PCl}$. However, in these patients, only a half achieved desirable EPA/AA ratio $(>0.40)$. Although, there was no significant difference, in patients with lower EPA/AA ratio tended to induce a coronary new lesions as comparing with higher EPA/AA ratio ( $25 \%$ vs. $14.3 \%$, respectively). As for the DHA/AA, no relationship with onset of new lesion was seen.

Conclusion: It is clarified that even if LDL concentration was in the targeted value, the management of EPA/AA was insufficient in a large number of patients after $\mathrm{PCl}$ procedure.

No conflict of interest 
ACUTE CORONARY SYNDROMES, MYOCARDIAL INFARCTION

\section{PREDICTION OF LONG-TERM MAJOR EVENTS SOON AFTER A FIRST ST-SEGMENT ELEVATIONMYOCARDIAL INFARCTION BY CARDIOVASCULAR MAGNETIC RESONANCE}

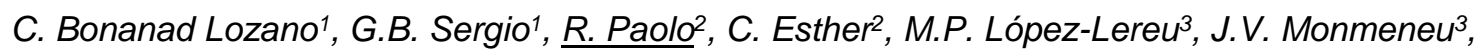
J. Gavara3 , F.J. Chorro', V. Bodi'

${ }^{1}$ Hospital Clínico de Valencia-INCLIVA, Cardiology, Valencia, Spain

${ }^{2}$ Hospital Clínico de Valencia, Cardiology, Valencia, Spain

${ }^{3}$ Hospital Clínico de Valencia-ERESA, Cardiology, Valencia, Spain

Aims: In post-STEMI patients CMR predicts combined clinical events. Its contribution for predicting long-term ME (cardiac death and non-fatal infarction) is unknown. We aimed to assess whether cardiovascular magnetic resonance (CMR) predicts long-term major events (ME) soon after ST-segment elevation myocardial infarction (STEMI).

Method: From 2004 to 2012 we prospectively recruited 546 STEMI patients. Left ventricular (LV) ejection fraction (LVEF, \%), infarct size (IS), edema, hemorrhage, microvascular obstruction and myocardial salvage were quantified by CMR at pre-discharge. During a mean follow-up of 840 days, $57 \mathrm{ME}$ events (10\%, 23 cardiac deaths, 34 non-fatal re-infarctions) were documented. Patients with ME displayed more depressed LVEF $(p<0.001)$, larger IS $(p<0.001)$, more extensive edema, hemorrhage and microvascular obstruction and less myocardial salvage $(p<0.05)$. CMR indexes were dichotomized according to the best cutoff values for predicting ME.

Results: In a comprehensive multivariate model, LVEF $<40 \%(2.3$ [1.2, 4.3], $\mathrm{p}=0.009)$ and IS $>30 \%$ of LV mass $(2.4[1.3,4.4], p=0.007)$ independently doubled the ME risk. The ME rates in patients with both LVEF $\geq 40 \%$ and IS $\leq 30 \%$ of LV mass ( $n=393$ ), in those with only one of them altered $(n=84)$ and in cases with both LVEF $<40 \%$ and IS $>30 \%(n=69)$ were $6 \%, 14 \%$ and $30 \%$ respectively $(p<0.001)$. Similar tendencies were observed regarding cardiac deaths $(2 \%, 6 \%$, $14 \%, \mathrm{p}<0.001)$ and re-infarctions $(4 \%, 8 \%, 16 \%, \mathrm{p}<0.001)$.

Conclusion: CMR predicts long-term ME soon after STEMI. A combined analysis of CMRderived LVEF and IS permits a robust stratification of patients' outcome.

No conflict of interest 
271

\title{
ACUTE CORONARY SYNDROMES, MYOCARDIAL INFARCTION
}

\section{INITIAL MANAGEMENT OF ST-ELEVATION MYOCARDIAL INFARCTION IN 2014: FROM GUIDELINES TO PRACTICES, SURVEY OF 176 FRENCH INTERVENTIONAL CARDIOLOGISTS}

\author{
E. Capilla, R. Poyet, F. Pons, G. Cellarier \\ Hôpital Sainte Anne, Cardiology, Toulon, France
}

\begin{abstract}
Aims: Coronary artery disease is the world leading cause of death. ST-elevation myocardial infarction (STEMI) is frequently the presenting feature of the disease. Real life management of STEMI has not recently been evaluated. The aim of the study was to describe STEMI management in France in 2014 and to compare it with European Society of Cardiology's guidelines.
\end{abstract}

Method: A multicentre, descriptive study was performed. A questionnaire was sent by e-mail to French interventional cardiologists. Demographic data of cardiologists, procedural aspects of percutaneous coronary intervention $(\mathrm{PCI})$, antithrombotic treatments and patient rehabilitation have been investigated.

Results: 176 answers were analysed. Averages of 209 STEMI were managed by centre, more often in academic hospitals than in general or private centres (respectively 51,32 and $17 \%$ of STEMI). On average, 5 interventional cardiologists participating in the on-call list by centre (from 2 to 14 ). About $23 \%$ of them are on-call more than 10 days per month. Used of anti-GP Ilbllla (34\% of cases) and thromboaspiration are not systematic but depend on patient's characteristics, according to guidelines. Radial access is favored in $85 \%$ cases and increased for 10 and even 5 years. Drug eluting stents are used in $62 \%$ of cases. Unfractioned heparin and enoxaparine account for more than $80 \%$ of anticoagulants treatments. Use of clopidogrel is as high as that of prasugrel or ticagrelor although clopidogrel is recommended in second-line. Cardiovascular rehabilitation is proposed to more than $50 \%$ of patients.

Conclusion: In spite of heterogeneity of STEMI management in 2014, real-life practices generally comply with current guidelines.

No conflict of interest 
211 - ACUTE CORONARY SYNDROMES, MYOCARDIAL INFARCTION

\title{
SEVERE ARRHYTHMIAS IN ACUTE PHASE OF STEMI IN THE ERA OF PRIMARY PCI PILOT DATA
}

\author{
L. Koc, M. Mikolaskova, J. Kanovsky, T. Ondrus, T. Novakova, A. Pleskova, M. Poloczek, \\ J. Spinar, P. Kala \\ The University Hospital Brno, Clinic of Internal Medicine - Cardiology, Brno, Czech Republic
}

Aims: To compare the incidence of severe arrhythmias in the first 48 hours of the onset of STEMI treated with $\mathrm{pPCl}$, according to its location and involvement of the right ventricle (RV).

Method: The study group consisted of 196 patients (average age 62 years, $62,2 \%$ males). It was divided into 3 subgroups according to the location of STEMI: A - Anterior STEMI $(n=93 ; 47,5 \%)$, B - Inferior STEMI without involvement of RV ( $n=82 ; 41,8 \%), C$ - Inferior STEMI with involvement of $R V(n=21 ; 10,7 \%)$. The involvement of RV was defined as $1 \mathrm{~mm}$ elevation of ST segment in V4R lead before $\mathrm{pPCl}$. Severe arrhythmias (ventricle fibrillation, sustained ventricle tachycardia, atrial fibrillation, second and third degree atrioventricular block) were recorded. The incidence of arrhythmias was statistically compared using chi-squared test.

Results: Combination of severe arrhythmias is described in Figure 1. No significant difference in the incidence of arrhythmias was observed (Figure 1).

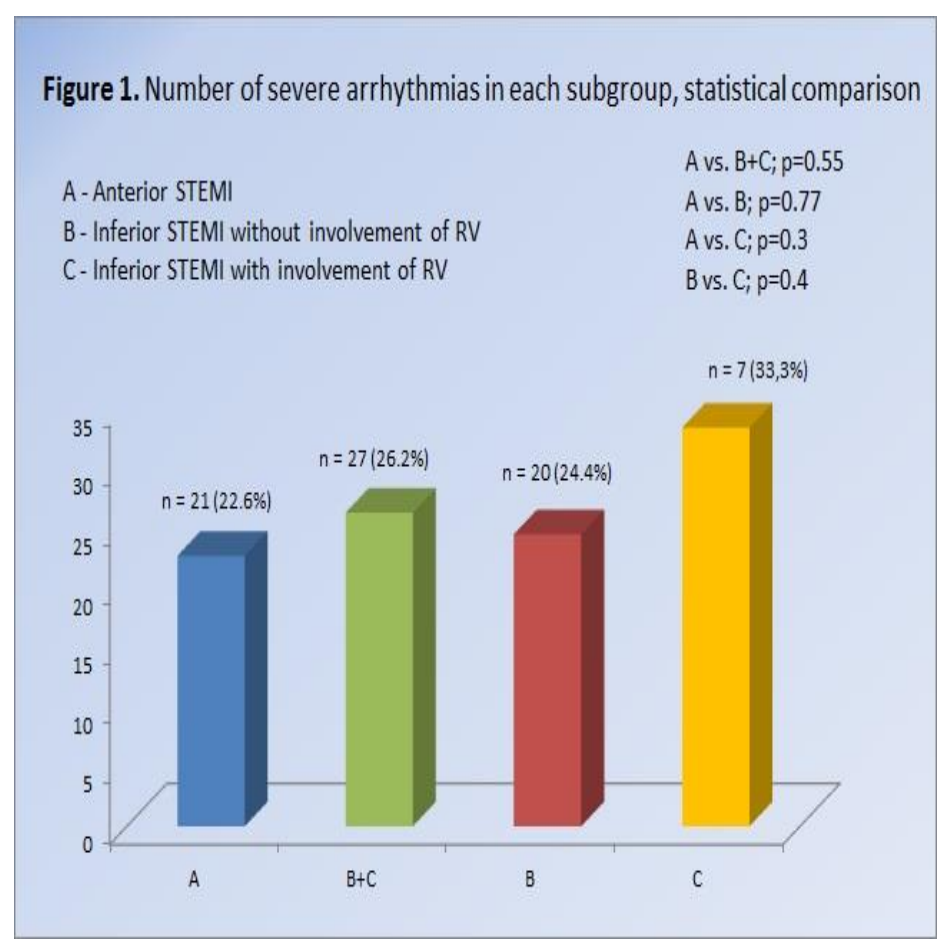


Conclusion: The occurrence of severe arrhythmias in acute phase of STEMI in patients treated with $\mathrm{pPCl}$ and in contrast to previous thrombolytic studies, was not dependent on the location of STEMI, nor on the involvement of the right ventricle. Supported by MH CZ - DRO (FNBr, 65269705), IGA MH CZ NT13767-4.

No conflict of interest 
273

\title{
ACUTE CORONARY SYNDROMES, MYOCARDIAL INFARCTION
}

\section{LEFT VENTRICULAR GLOBAL LONGITUDINAL SYSTOLIC STRAIN PREDICTS ADVERSE REMODELING AND SUBSEQUENT CARDIAC EVENTS IN PATIENTS WITH ACUTE MYOCARDIAL INFARCTION WITH PRIMARY PCI}

\author{
M. Izquierdo, M. Martín, C. Méndez, B. Marí, C. Belleyo, A. De la Rosa, A. Duque, J. Lacalzada, \\ I. Laynez \\ Hospital Universitario de Canarias, Cardiology, La Laguna, Spain
}

Aims: The aim of our study was to determine whether global longitudinal systolic strain (GLS) predicts adverse LV remodeling and cardiac events.

Method: This cohort study included 103 consecutive AMI patients treated with primary PCI. Clinical data and GLS, global circunferencial strain and radial strain were recorded using twodimensional speckle-tracking echocardiography of the LV. At 6-month and 3-year follows-ups, patients were grouped by presence or absence of adverse LV remodeling.

Results: Of 97 patients (mean age $56 \pm 12$ years; 76 men), 38 showed LV remodeling. Diabetes mellitus [odds ratio (OR 1,95\% confidence interval (CI) 1,2-4,8 $p=0,05$ ], peak troponin I (OR 1,2, $95 \% \mathrm{Cl} 1,1-1,3 \mathrm{p}=0,004)$, and GLS (OR 1,6, 95\% Cl 1,3-2,3, $\mathrm{p}=0,009$ ) independently predicted LV remodeling. During follow up (22,8 $\pm 12,3$ months), 20 patients suffered adverse events, which were independently predicted by GLS alone (OR 4,9, 95\% Cl 1,7-13,9, p=0,002). Optimal GLS cutoffs for predicting adverse LV remodeling and cardiac events were $>-12,46 \%$ [area under receiver operating-characteristic curve (AUC) 0,88, 95\% Cl 0,79-0,96, p<0,001] and $>-9,27 \%$ (AUC 0,86, 95\% Cl 0,64-0,98, p<0,001), respectively.

Conclusion: GLS measured is an excellent predictor of adverse LV remodeling and cardiac events in patients with AMI.

No conflict of interest 


\title{
ACUTE CORONARY SYNDROMES, MYOCARDIAL INFARCTION
}

\section{MARKERS OF ENDOTHELIAL ACTIVATION AND COAGULATION PREDICT MAJOR ADVERSE CARDIOVASCULAR EVENTS IN PATIENTS WITH ACUTE CORONARY SYNDROME: INSIGHTS FROM AIRACOS STUDY}

\author{
${ }_{\text {P. Avanzas }}{ }^{1}$, A. Dominguez-Rodriguez ${ }^{2}$, P. Abreu-Gonzalez ${ }^{3}$, S. Rodriguez ${ }^{4}$, R. Juarez-Prera² \\ ${ }^{1}$ Hospital Universitario Central de Asturias, Cardiology, Oviedo, Spain \\ ${ }^{2}$ Hospital Universitario de Canarias, Cardiology, Santa Cruz de Tenerife, Spain \\ 3Universidad de La Laguna, Departamento de Ciencias Medicas Básicas Unidad de Fisiología, \\ Santa Cruz de Tenerife, Spain \\ ${ }^{4}$ AEMET, Centro de Investigación Atmosférica de Izaña CIAl, Santa Cruz de Tenerife, Spain
}

\begin{abstract}
Aims: Exposure to particulate matter air pollution (PM) has been associated with cardiovascular diseases, and patients with acute coronary syndromes (ACS) may be especially vulnerable. The aim of the study was to examine whether exposure to particulate matter air pollution (PM), markers of inflammation, endothelial activation and coagulation predict one year major adverse cardiovascular events (MACE) in patients with ACS.
\end{abstract}

Method: We included 307 consecutive ACS patients admitted into a tertiary care hospital, and assessed the average concentrations of different sizes of PM10, PM2.5 and black carbon (BC), the concentrations of gaseous pollutants and meteorological parameters from 1 day up to 7 days prior to admission. Levels of blood markers for systemic inflammation (hsCRP), endotelial activation (sVCAM-1) and coagulation (sCD40L) were measured. Patients were clinically followed during one year and the occurrence of MACE was registered in all patients.

Results: Twenty one patients $(6,8 \%)$ showed MACE at one year follow-up. Patients who developed MACE, had higher BC concentrations $(948.4 \pm 147.4$ vs $858.8 \pm 202.5 \mu \mathrm{g} / \mathrm{m} 3, \mathrm{p}=0.01)$, sVCAM-1 (735.7 [496.7-875 vs 389.4 [163-664.8 ]pg/mL]) and SCD40L (6.4 [3.4-7.7] vs 2.7 [1.16.1], $p=0.009$ ), but not hs-CRP levels (8.6 [5-18.6]), compared to patients who did not. After adjustment with binary logistic regression, sVCAM-1 $(p=0.003)$ and $S C D 40 L(p=0.007)$ were independent predictors of MACE. The association between MACE and markers of endothelial activation and coagulation remained significant even when BC exposure was introduced in the multivariate model.

Conclusion: In ACS patients, markers of endothelial activation and coagulation, but not BC exposure, are associated with MACE at one year follow-up.

No conflict of interest 


\title{
ARRHYTHMIAS, EKG
}

\section{ANOMALOUS COURSE OF THE LEFT MAIN CORONARY ARTERY BETWEEN THE MAIN ARTERIES AS A CAUSE OF CARDIAC ARREST}

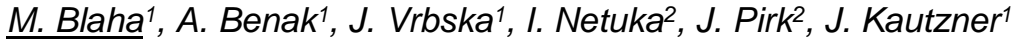 \\ ${ }^{1}$ Institute for Clinical and Experimental Medicine, Cardiology, Prague, Czech Republic \\ ${ }^{2}$ Institute for Clinical and Experimental Medicine, Cardiovascular Surgery, Prague, \\ Czech Republic
}

Aims: Certain types of coronary arteries anomalies are associated with increased risk of sudden cardiac death.Surgical correction is the method of choice.

Method: We report on a case of 34-year-old female without history of cardiac disease,hospitalized after resuscitation for cardiac arrest during jogging. The first documented rhythm was ventricular fibrillation,terminated by the first defibrillation attempt.On admission,the patient presented with ST segment elevation in the precordial leads.Immediate coronarography was performed with normal right coronary artery. Due to failed selective cannulation of the left coronary artery(LCA), non-selective angiography was performed.LCA was described as normal.Echocardiography documented left ventricular(LV) systolic dysfunction with EF $20 \%$. The patient was transferred to our department for consideration of emergency mechanical support.Complex conventional treatment led to stabilisation of the hemodynamics, and,gradually,to normalisation of LV function. The patient survived the acute period with milder memory impairment and dysarthria.During reconvalescence,ventricular fibrillation once relapsed and was terminated immediately by an external shock.

Results: Subsequent CT coronarography showed anomalous origin of LCA from the right coronary sinus, and its course between both main arteries. The final diagnosis was cardiac arrest due to intermittent compression of LCA. Forty days after admittion, when neurological rehabilitation was completed,cardiac surgery was performed with roofing of the LCA to the left coronary sinus. Implantation of a cardioverter/defibrillator was not indicated.The patient was without any cardiovascular events during fifteen-month follow-up.She lives an active life and successfully rehabilitates the residual short-term memory disorder.

Conclusion: This case highlights the need for complex evaluation of survivors of out-of-hospital cardiac arrest in a specialized center. This allows to individualize terapeutical approach even in less frequent causes of cardiac arrest.

No conflict of interest 


\section{PATIENT WITH CHEST PAIN AND WIDE QRS. IMPORTANCE OF ECG DIAGNOSIS. A CASE REPORT}

V. Bonilla ${ }^{1}$, P. Escribano ${ }^{2}$, R. Garcia ${ }^{1}$, A. Garcia-lledo ${ }^{1}$

${ }^{1}$ Hospital Principe De Asturias, Cardiology Department, Alcala De Henares, Spain

${ }^{2}$ Hospital Principe De Asturias, Endocrinology Department, Alcala De Henares, Spain

Aims: The presence of LBBB hinders the evaluation of patients with chest pain. A systematic reading of the ECG can be crucial.

Method: We report the case of a patient attending Emergency with chest pain and unknown LBBB image in the ECG.

Results: 38 year old male patient. No specific treatment or relevant personal or family history. The patient reported 2 days long episodes of atypical, short duration, central chest pain, without clear correlation to efforts. Likewise, the patient reports occasional palpitations and syncope one week earlier while sitting. Given the history of chest pains and assuming the ECG abnormality as LBBB, stress echocardiogram is performed resulting negative for ischemia. The correct analysis of the ECG was decisive. Detailed ECG analysis led us to find a short PR (PR $<120 \mathrm{msec}$ ) and presence of initial delta wave compatible with WPW. The exercise ECG showed the narrowing of QRS at elevated HR (HR> $150 \mathrm{bpm})$ keeping the presence of delta wave in some beats, making the existence of an accessory pathway more likely than a conduction disorder. Finally electrophysiology study was realized confirming the presence of an accessory pathway.

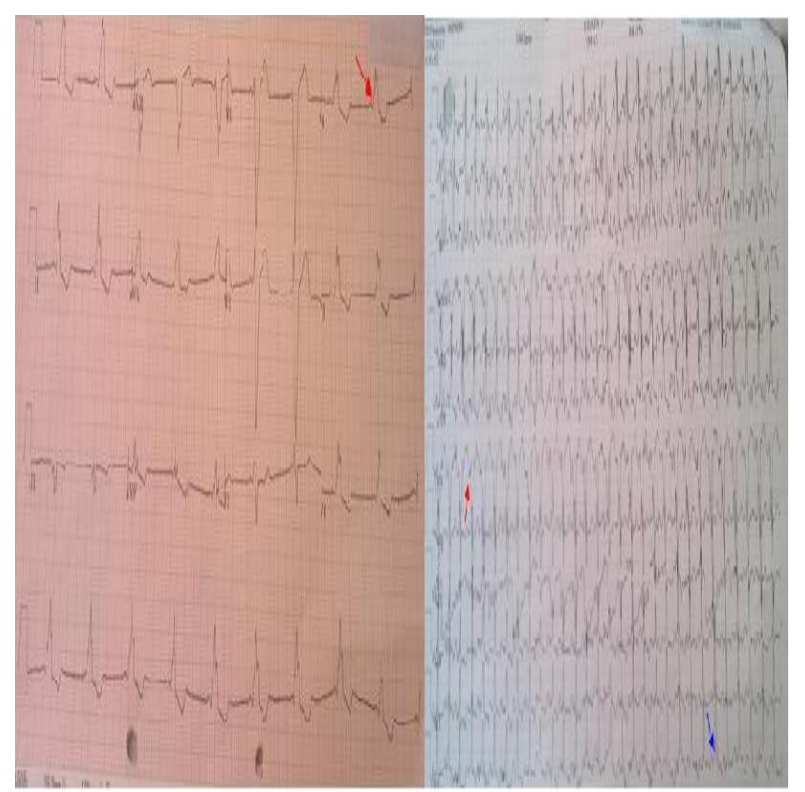

Conclusion: The WPW syndrome can mislead ECG analysis. Delta wave can simulate Q waves of myocardial necrosis and ventricular repolarization abnormalities can mimic ischemic changes, as well as imitate intraventricular conduction disorders as in our case. It is important to always conduct a detailed and systematic ECG reading, both at rest and in relation to dynamic changes. 
No conflict of interest 
277

\title{
ARRHYTHMIAS, EKG
}

\section{GAP BETWEEN UNDIAGNOSED AND REGISTERED ATRIAL FIBRILLATION IN ADULTS AND INEFFECTIVENESS OF ORAL ANTICOAGULATION TREATMENT WITH VITAMIN $K$ ANTAGONIST THERAPY}

\author{
J.L. Clua-espuny', A. Panisello-Tafalla ${ }^{1}$, G. Procida-Cervera ${ }^{1}$, A. Gonzalez-Henares ${ }^{1}$, L. Queralt- \\ Tomas $^{1}$, R. Ripolles-Vicente ${ }^{1}$, C. Lopez-Pablo², F. Ferreira-Caro ${ }^{1}$, I. Lechuga-Duran ${ }^{3}$ \\ ${ }_{1}^{1}$ Institut Catala de la Salut, Primary Care, Tortosa, Spain \\ ${ }^{2}$ Molecular Biology and Research Section- Hospital de Tortosa Verge de la Cinta, IDIAP, Tortosa, \\ Spain \\ ${ }^{3}$ Institut Catala de la Salut, Cardiology. Hospital Verge de la Cinta, Tortosa, Spain
}

Aims: This study aimed to examine the effectiveness of the use of oral anticoagulation (OAC) medication, recommended by national guidelines for stroke prevention, but reportedly underused in AF patients with moderate to high stroke risk.

Method: A multicentre and cross-sectional study of undiagnosed AF among out-of-hospital patients over 60yearsold was carried out, visiting 3,638 patients at primary health centres or at home for AF diagnosis using the IDC-10 classification. The main outcome measures were $\mathrm{CHADS}_{2} / \mathrm{CHA}_{2} \mathrm{DS}_{2}$-VASc, HAS-BLED scores, cardiovascular comorbidity, pharmacological information, TTR and SAMe-TT2R2 scores

Results: The main findings were: undiagnosed AF in $26.44 \%$ of cases; $31.04 \%$ registered with AF but not using OAC despite $95.6 \%$ having a CHA2DS2VASC $\geq 2$ score; a risk of bleeding in important subgroups using OAC without indication (37.50\% CHA2DS2VASC $<2$ score); the use of OAC with TTR $<60 \%(33.1 \%)$, of whom $47.6 \%$ had a HAS-BLED score $\geq 3$. Thus, $35.4 \%$ of the expected AF prevalence achieved an optimal time in the therapeutic range.

Conclusion: The expected AF prevalence was 10.9\% (n 5267), but the registered prevalence was $7.5 \%$ (n 3638). Only $35.04 \%(\mathrm{Cl}=95 \%, 33.7-36.3)$ of $\mathrm{AF}$ patients treated with vitamin $\mathrm{K}$ antagonists (VKAs) achieve the goal of TTR $>60 \%$.

No conflict of interest 
ACS, ACUTE MYOCARDIAL INFARCTION

\title{
CLINICAL PROFILE OF ADULT PATIENTS WITH POSITIVE HIGH SENSITIVITY TROPONIN I (HSTNI) LEVELS ADMITTED IN THE MEDICAL CITY, PHILIPPINES
}

\author{
K.G. Floren ${ }^{1}$, R. Ramboyong ${ }^{2}$ \\ ${ }^{1}$ The Medical City- Philippines, Department of Internal Medicine- Section of Adult Cardiology, \\ Quezon City, Philippines \\ ${ }^{2}$ The Medical City- Philippines, Department of Internal Medicine- Section of Adult Cardiology, \\ Pasig, Philippines
}

\begin{abstract}
Aims: ARCHITECT STAT hsTnl, introduced in our institution in May 2014, is a new genderspecific high sensitive troponin assay which aids in the early diagnosis of acute coronary syndrome. This study aims to describe the clinical profile of all hsTnl-positive adult patients admitted in The Medical City.
\end{abstract}

Method: All admitted patients from July 2014 to December 2014 with positive hsTnl (>15.6 pg/ml for females and $34.2 \mathrm{pg} / \mathrm{ml}$ for males) were included. Demographics, co-morbidities, chief complaint, 12L-electrocardiogram, 2D-echocardiogram, renal function, treatment received and outcomes were recorded and analyzed.

Results: Patients were mostly Filipino (97.66\%), males (55\%) with a mean age of 65 years and body mass index of $24.87 \mathrm{~kg} / \mathrm{m} 2$ coming in due to difficulty of breathing $(35.09 \%)$ or chest pain $(23.10 \%)$. Majority were hypertensive $(80.7 \%)$ and diabetic $(42.98 \%)$ and were treated as acute myocardial infarction with lesions predominantly involving the anterior wall (23.69\%) by 2Dechocardiogram and anterolateral wall $(45.83 \%)$ by $12 \mathrm{~L}$-electrocardiogram and received optimal medical management (55.55\%) and revascularization (19.95\%). Increasing hsTnl levels were observed in progressive renal dysfunction but levels decrease in dialysis patients. These were more complicated by non-cardiac diagnosis such as sepsis.

Conclusion: Positive and higher levels of hstnl were detected in hypertensive, male patients with a mean age of 65 presenting with difficulty of breathing, primarily treated as acute myocardial infarction and were commonly associated with anterior lesions and poor prognosis.

No conflict of interest 


\title{
THROMBOPHILIA AND OTHER CONDITIONS ASSOCIATED WITH ACUTE FORMS OF CARDIOVASCULAR DISEASE
}

\author{
J. Cmrečnjak ${ }^{1}$, M. Kursar ${ }^{2}$, M. Gradišer ${ }^{3}$ \\ ${ }^{1}$ County Hospital Čakovec-Croatia, Intensive Cardiology Care Unit and Cardiology, Čakovec, \\ Croatia \\ ${ }^{2}$ County Hospital Čakovec- Croatia, Hematology, Čakovec, Croatia \\ ${ }^{3}$ County Hospital Čakovec- Croatia, Endocrinology and Diabetes, Čakovec, Croatia
}

Aims: In cardiology, we are witnessing an increase in cases of ACS in younger patients, i.e. in patients with no typical risk factors. Only a small number of studies examined the influence and association of genetic factors and ACS with angiographically normal coronary arteries.

Development of more sensitive tests allowed precise biochemical definition of hypercoagulable states. Molecular technology allows us to perform genetic analysis.

Method: Myocardial infarction (MI)classification recognizes five types of infarction depending on pathophysiological, clinical, and prognostic factors. MI with angiographically normal coronary arteries (MINCA) falls under nonatherosclerotic causes. There are several mechanisms for explanation of MINCA: latent atherosclerosis, vasospasm, thrombosis in situ and a hypercoagulable state, embolization, and inflammation. Differential diagnosis of such acute coronary events includes myocarditis, stress cardiomyopathy, and Takotsubo syndrome.Beside the traditional immunological and functional tests examining the hereditary lack of antithrombin and protein $\mathrm{C}$ and $\mathrm{S}$, we can also apply genetic tests that verify mutations related to thrombogenesis:factor $\mathrm{V}$ Leiden, prothrombin mutation, PAI-1 polymorphism, MTHFR.

Results: During 2013.we treated eight patients that had acute coronary syndrome with angiographically normal coronary arteries - MINCA. In six patients we perform a genetic analysis and cardiac MRI and proved gene mutations related to thrombogenesis.

Conclusion: Since thrombophilia is hereditary,genetic testing can benefit not only the person with a particular polymorphism but also other close members of the family. Being aware of a genetic predisposition can prevent exposure to factors that can facilitate thrombogenesis. Thus, genetic analysis must be performed in younger patients that had cardiovascular, cerebrovascular, and peripheral thromboembolic events but have no typical risk factors.

No conflict of interest 


\title{
IMPACT OF ADMISSION RED CELL DISTRIBUTION WIDTH ON 30 DAYS CLINICAL OUTCOMES IN STEMI PATIENTS UNDERGOING PRIMARY PCI
}

\author{
V. Dedovic ${ }^{1}$, I. Mrkonjic ${ }^{2}$, Z. Mehmedbegovic ${ }^{1}$, M. Zivkovic ${ }^{1}$, D. Milasinovic ${ }^{1}$, M. Asanin ${ }^{3}$, \\ V. Kanjuh ${ }^{4}$, V. Vukcevic ${ }^{1}$, G. Stankovic ${ }^{1}$, J. Dobras $^{1}$ \\ ${ }^{1}$ Department of Cardiology- Clinical Center of Serbia, Catheterisation laboratory, Belgrade, Serbia \\ ${ }^{2}$ Department of Cardiology- Clinical Center of Serbia, Cardiology, Belgrade, Serbia \\ ${ }^{3}$ Department of Cardiology- Clinical Center of Serbia, Emergency Department, Belgrade, Serbia \\ ${ }^{4}$ Serbian Academy of Sciences and Arts, Board on Cardiovascular Pathology, Belgrade, Serbia
}

Aims: The aim of our study was to evaluate if higher red cell distribution width values at admission (aRDW) correlate with major adverse cardiovascular events (MACE) at 30 days in pPCI STEMI patients.

Method: We analyzed 514 consecutive STEMI patients treated with $\mathrm{pPCl}$ in high volume $\mathrm{pPCl}$ centre. Patients are divided according to aRDW median (12.9) in Group $1-$ aRDW $<12.9$ and Group 2 - aRDW $\geq 12.9$. MACE was defined as a composite of cardiovascular death, recurrent myocardial infarction (MI), stent thrombosis (ST) and new/worsening NYHA Class IV heart failure(HF).

Results: Patients in group 1 were younger and had higher estimated glomerular filtration rate. Other clinical and angiographic characteristics were similar between two groups. Overall 30 days MACE was $7.4 \%$. Patients in Group 2 higher rate of MACE, mortality and HF at 30 days. 30-days MI rate in this group was also higher but did not reach statistical significance. (Table 1) However, after adjustment for other clinical and angiographic variables aRDW was not independent predictor of 30 days MACE.

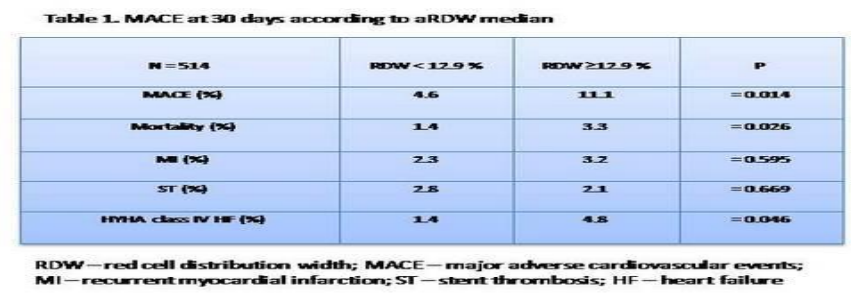

Conclusion: Higher aRDW values in STEMI patients are associated with elevated risk of MACE after $\mathrm{pPCl}$, but are not independent predictor of 30 days MACE.

No conflict of interest 
281

ACS, ACUTE MYOCARDIAL INFARCTION

\title{
IMPACT OF PERIPROCEDURAL KIDNEY INJURY DEFINED BY ACUTE KIDNEY INJURY NETWORK CRITERIA ON 5-YEAR MORTALITY AFTER PRIMARY PCI
}

\author{
$\underline{\text { J. Dobras }}^{1}$, D. Milasinovic ${ }^{1}$, V. Pavlovic ${ }^{1}$, I. Zivkovic ${ }^{1}$, V. Dedovic ${ }^{1}$, Z. Mehmedbegovic ${ }^{1}$, \\ V. Vukcevic ${ }^{1}$, V. Kanjuh', G. Stankovic ${ }^{1}$ \\ ${ }^{1}$ Clinical Center of Serbia, Department of Cardiology, Belgrade, Serbia \\ ${ }^{2}$ Serbian Academy of Sciences and Arts, Board on Cardiovascular Pathology, Belgrade, Serbia
}

Aims: Limited information is available regarding the use and clinical relevance of the acute kidney injury network (AKIN) classification in mortality prediction in STEMI patients treated with primary PCl.

Method: We analyzed 1722 patients from a registry of a high-volume primary $\mathrm{PCl}$-center from the years 2009-2010. AKI was defined according to the AKIN criteria as: stage 1 (rise in serum creatinine $(\mathrm{SCr}) \geq 0.3 \mathrm{mg} / \mathrm{dl}$ or $150-200 \%$ from baseline), stage 2 (rise in SCr $200-300 \%$ ) and stage 3 (rise in SCr $\geq 300 \%$ or if baseline $\mathrm{SCr} \geq 4 \mathrm{mg} / \mathrm{dl}$ then rise by $\geq 0.5 \mathrm{mg} / \mathrm{dl}$ ).

Results: Overall incidence of AKI as defined by AKIN criteria was $10.5 \%(n=181)$ and overall mortality at median follow-up of 4.8 years (IQR, $4.4-5.2)$ was $19 \%(n=328)$. Stage 1 AKI was documented in 161 patients (9.4\%), stage 2 in 13 patients (0.75\%) and stage 3 in 7 patients $(0.4 \%)$. Cumulative mortality at 5 years differed significantly between the groups: $16.6 \%$ in patients with no AKI vs. $37.3 \%$ in patients with stage $1 \mathrm{AKI}$ vs. $53.8 \%$ in stage 2 and $71.4 \%$ in stage 3 (log-rank test $<0.001$ ). Unadjusted Cox regression model showed three-fold higher risk of 5 -year mortality (HR 3.005, 95\% $\mathrm{Cl} 2.313-3.904, \mathrm{p}<0.001)$ for patients with AKI compared to no AKI. After adjusting for key baseline characteristics, AKI tended to independently predict very long-term mortality (HR 1.373, 95\% Cl 0.989-1.957, $\mathrm{p}=0.058)$.

Conclusion: In primary PCI patients, acute kidney injury is associated with higher rate of 5-year mortality. Furthermore, AKIN criteria stratify patients according to the increasing mortality risk.

No conflict of interest 
282

ACS, ACUTE MYOCARDIAL INFARCTION

UNUSUAL CAUSE OF MYOCARDIAL INFARCTION (STEMI)

P. Jebavy

Kardiologie na Bulovce s.r.o., Prague, Czech Republic

Aims: Cause study of 64 years old man with 14 year history of aortocoronary bypass (ACB), LIMA ad LAD and autovenous ad RCA.

Method: Patient was oligosymptomatic, but in routine investigation positive findings for ischemia in myocardial SPECT were found. Patient was determined to coronary angiography (CAG) with these findings: LAD was closed, LCX has non-significant stenosis in proximal part in angulation. RCA was closed proximally. LIMA ad LAD flow was good, with heterocollaterals to RCA. Autovenous ACB ad RCA was degenerated with residual TIMI $1^{\circ}$ flow.

Results: Treatment strategy: $\mathrm{ACB}$ ad RCA was appointed to PCI treatment. Guide wire passed easily to periphery of RCA, but closed the flow completely. Balloon didn't pass to ACB. PCI was finished as unsuccessful. After 10 hours, patient claimed severe chest pain. ST elevation in ECG, highly elevated cardiac enzyme and CRP together with hypotension and slight worsening of LVEF determine STEMI. As culprit lesion was dedicated ACB unsuitable for PCI treatment and was assessed to medical treatment. Patient was dismissed in good condition. After 10 days, patient refered progression of chest pain. Repeated CAG showed proximal trombotic obstruction of LCX. Catch of a fragment of broken tip of diagnostic catheter from first CAG was the reason of thrombosis. Direct stenting $(3,0 \times 18 \mathrm{~mm})$ was a method of choice and flow to periphery of LCX was restored.

Conclusion: We demonstrate very rare cause of iatrogenic STEMI after failure of instrument. After first incorrect decision in acute situation, we treat patients interventionally. Angina or worsening of LVEF haven't been seen.

No conflict of interest 


\title{
DURATION OF DUAL ANTIPLATELET THERAPY IN RUSSIAN PATIENTS AFTER MYOCARDIAL INFARCTION (EPICOR-RUS STUDY)
}

\author{
Y. Khomitskaya ${ }^{1}$, M. Ruda ${ }^{2}$, O. Averkov ${ }^{3}$, O. Apanova ${ }^{1}$ \\ ${ }^{1}$ AstraZeneca, Medical, Moscow, Russia \\ ${ }^{2}$ Russian Cardiology Research and Production Complex, Urgent Cardiology, Moscow, Russia \\ ${ }^{3}$ People's Friendship University of Russia, Therapy Department, Moscow, Russia
}

Aims: To describe long-term antithrombotic management patterns in a real-life setting for patients hospitalized with an acute coronary syndrome (ACS).

Method: EPICOR-RUS (NCT01373957) was a Russian urban multi-centre (35 hospitals), observational cohort study, that described short- and long term (up to 2 years) antithrombotic management patterns in a total of 599 patients (pts) who had been hospitalized for ACS within 24 hours of symptom onset, and had a discharge diagnosis of either unstable angina (UA) $(n=77)$, non-ST-elevation myocardial infarction (NSTEMI) $(n=148)$ or STEMI $(n=374)$. This analysis focuses duration on dual antiplatelet therapy (DAPT).

Results: In the STEMI group $89.9 \%(n=337)$ were prescribed DAPT: $97.9 \%(n=236)$ of pts who underwent percutaneous coronary intervention $(\mathrm{PCl})$ and $75.4 \%(\mathrm{n}=101)$ of medically managed pts. At 6 months $92.2 \%$ of STEMI-PCI and $61.3 \%$ of medically managed STEMI pts still took DAPT. Only $72.4 \%$ of STEMI-PCI and $39.5 \%$ of medically managed STEMI pts received DAPT at 12 months. In the NSTEMI group, $78.2 \%(n=115)$ were prescribed DAPT: $92.6 \%(n=50)$ of pts who underwent $\mathrm{PCl}$ and $69.9 \%$ ( $\mathrm{n}=65)$ of medically managed pts. At 6 months, $93.2 \%$ of NSTEMI-PCI and $69.8 \%$ of medically managed NSTEMI pts still took DAPT. Only $77.3 \%$ NSTEMI-PCI and $26.4 \%$ of medically managed NSTEMI pts received DAPT at 12 months.

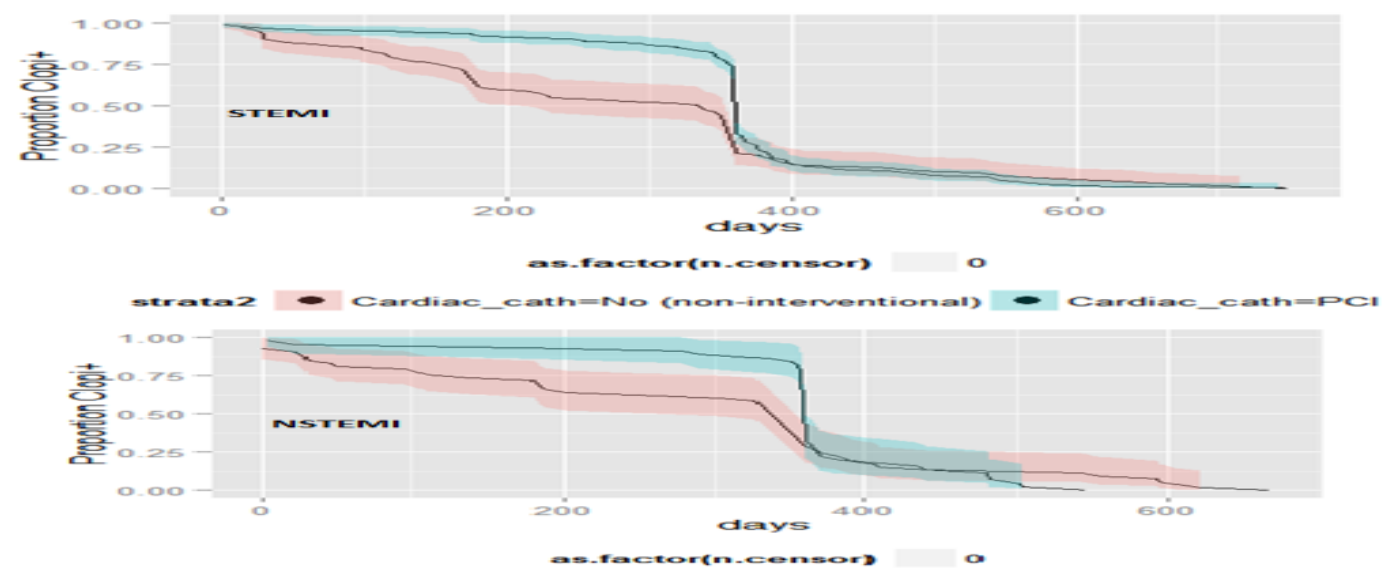

Conclusion: Compliance with DAPT in medically managed STEMI and NSTEMI patients is worse than in patients who underwent $\mathrm{PCl}$ and requires further improvement.

Conflict of Interest Disclosure Statement:

Yunona Khomitskaya and Olga Apnova are AstraZeneca company employees. EPICOR-RUS 
study is funded by AstraZeneca. 
ACS, ACUTE MYOCARDIAL INFARCTION

\title{
ANTIPLATELET THERAPY PATTERNS IN NON ST-ELEVATION MYOCARDIAL INFARCTION IN URBAN RUSSIA (EPICOR-RUS STUDY)
}

\author{
Y. Khomitskaya ${ }^{1}$, M. Ruda ${ }^{2}$, O. Averkov ${ }^{3}$, O. Apanova $^{4}$ \\ 1, Moscow, Russia \\ ${ }^{2}$ Russian Cardiology Research and Production Complex, Urgent cardiology, Moscow, Russia \\ ${ }^{3}$ People's Friendship University of Russia, Therapy, Moscow, Russia \\ ${ }^{4}$ AstraZeneca, Medical, Moscow, Russia
}

Aims: To describe antiplatelet therapy patterns in patients (pts) with non ST-elevation myocardial infarction (NSTEMI) in urban Russian hospitals.

Method: EPICOR-RUS (NCT01373957) was a Russian urban multi-centre (35 hospitals), observational cohort study that described short- and long term (up to 2 years) antithrombotic management patterns in a total of 599 pts who had been hospitalized for acute coronary syndrome within 24 hours of symptom onset and who had a discharge diagnosis of either unstable angina $(n=77)$, NSTEMI $(n=147)$ or ST-elevation myocardial infarction $(n=375)$. This analysis focuses on the percutaneous coronary intervention (PCl) and anti-platelet treatment patterns in the NSTEMI group.

Results: Patient sample is based on the NSTEMI group ( $n=147) .54(36.7 \%)$ pts underwent PCI during hospitalization; 68 (46.3\%) pts received aspirin monotherapy, 4 (2.7\%) - clopidogrel monotherapy and $19(12.9 \%)$ - dual anti-platelet therapy (DAPT) at the pre-hospital stage; 32 $(21.8 \%)$ pts received aspirin monotherapy, $3(2 \%)$ - clopidogrel monotherapy and $109(74.1 \%)$ DAPT in hospital. Three (2\%) pts received GP Ilb/llla inhibitors. Ten (6.8\%) pts received loading dose of clopidogrel $600 \mathrm{mg}$ and 45 (30.8) recieved $300 \mathrm{mg} .30(20.4 \%)$ pts were recommended at discharge to take 75 and $100 \mathrm{mg}$ aspirin monotherapy (23.3\% and $56.7 \%$, respectively). At discharge $2(1.9 \%)$ were prescribed $75 \mathrm{mg}$ clopidogrel monotherapy and $115(78.2 \%)$ - DAPT with $75 \mathrm{mg}$ of clopidogrel and aspirin.

Conclusion: The findings that only $74.1 \%$ received DAT in hospital and $78.2 \%$ were prescribed DAT highlight that there is room for improvement in this area in urban Russian hospitals.

Conflict of Interest Disclosure Statement: Khomitskaya and Apanova - AstraZeneca employees, the study is funded by AstraZeneca company 
285

ACS, ACUTE MYOCARDIAL INFARCTION

PERI-OPERATIVE TAKOTSUBO CARDIOMYOPATHY: A CASE SERIES

J. Cerny, S. Kee, F. Goravanchi, E. Rebello, A.M. Kowalski

Department of Anesthesiology \& Peri-Operative Medicine, MD Anderson Cancer Center, Houston, Texas, USA

Introduction: Of hospital admissions in the U. S. for acute coronary syndrome, Takotsubo Cardiomyopathy (TC) accounts for 1-2\%. Characterized by angina, ST abnormalities, elevated troponins, ventricular asynergy, $\mathrm{CHF}$, and a decreased EF the pathophysiology demonstrates myocardial weakness and midventricular or apical ballooning in the absence of CAD. Three institutional cases are being presented in which the cardiac symptoms lead to the diagnosis of TC.

Case one:

69 year old female presents for cystoscopy/transurethral resection of bladder tumor/exam under anesthesia/ureteroscopy. Post-operatively, she c/o angina.

\section{Case two:}

57 year old female with multinodular goiter presents for total thyroidectomy. Post-operatively, she developed $9 / 10$ chest pain.

\section{Case three:}

63 year old female with ovarian carcinoma presents for exploratory laparotomy, lysis of adhesions, and tumor reduction surgery. Post-operatively, the patient reported angina.

Diagnostic Findings For Each Case Discussion:

The etiology of TC is unclear; however, activation of the sympathetic nervous system is likely due to high levels of circulating catecholamines. Catheterization is required to confirm diagnosis. Pathognomonic for TC is LV apical wall ballooning (not related to any specific coronary artery territory) in the presence of normal coronary arteries. Since TC is more common than once thought, it is important to have a heightened level of awareness regarding the stress-induced cardiomyopathy within a differential.

No conflict of interest 


\section{ACTIVATING ANTI-OXIDANT ENZYMES INHIBIT MYOCARDIAL ISCHEMIA/REPERFUSION INJURY BY NATURAL COMPOUND DERIVATIVE}

H.C. Ku ${ }^{1}$, Y.H. Kuo ${ }^{2}$, M.J. Su${ }^{1}$

${ }^{1}$ National Taiwan University, Institute of Pharmacology, Taipei, Taiwan

${ }^{2}$ National Taiwan University, Department of Chemistry, Taipei, Taiwan

Aims: Excessive reactive oxygen species release impairs the function and structure of antioxidant enzyme systems and induces cell death. The free radical scavenging ability of compound in chemical reaction may not always equal to the response in vivo. We aim to compare and investigate the action between caffec acid (CA) and its derivative, 36-12, on acute myocardial infarction.

Method: Rats were subjected to myocardial ischemia/reperfusion (I/R) to induce oxidative stress. Proteins expression was detected. Cardiac function was measured by pressure-volume loop.

Results: We demonstrated that CA and 36-12 exert the same ability to scavenge DPPH in vitro. However, 36-12 is more efficiency than CA in protecting against oxidative stress in vivo. After rat subjected to I/R stress, 36-12 was shown to attenuate oxidative injury more than CA did, accompanied with a prominent elevation of AKT and HO-1 expression and a preservation of mnSOD and catalase activity. When inhibiting AKT or HO-1 pathway, 36-12 loss its ability to recover mnSOD and catalase activity. In addition, rats treated with 36-12 had better cardiac performance after I/R stress.

Conclusion: We provide a new insight into this therapeutic potential for CA derivative in myocardial I/R injury.

No conflict of interest 


\title{
RECURRENT INTRAVENTRICULAR THROMBUS SIX MONTHS AFTER ST-ELEVATION MYOCARDIAL INFARCTION IN A DIABETIC MAN: A CASE REPORT
}

\author{
B. Marí López, C. Belleyo, C. Méndez, M. Martín, M.M. Izquierdo, A. De la Rosa, A. Duque, \\ J. Lacalzada, I. Laynez \\ Hospital Universitario de Canarias, Department of Cardiology- Cardiac Imaging Laboratory, \\ Santa Cruz De Tenerife, Spain
}

Aims: Percutaneous coronary intervention (PCI) with placement of a drug-eluting stent in patient with ST-elevation myocardial infarction (STEMI) is a common procedure, and always requires treatment with dual antiplatelet therapy. It is sometimes necessary to add oral anticoagulation (ACo) therapy, which increases the risk of bleeding.

Method: A 66-year-old diabetic man with a history of gastrointestinal bleeding was admitted with an STEMI $72 \mathrm{~h}$ of evolution. Electrocardiography showed ST segment elevation in the inferior leads and $Q$ waves in the inferior and anterior leads. Coronary angiography showed chronic occlusion of the mid-left anterior descending coronary artery, and acute occlusion of the mid-right coronary artery. He was treated by $\mathrm{PCl}$, with placement of a drug-eluting stent in the right coronary artery. Transthoracic echocardiography (TTE) showed abnormal left ventricular (LV) contractility and a LV thrombus. The patient was discharged on dual antiplatelet therapy and ACo therapy.

Results: Four months later, TTE showed absence of LV thrombus. Given the high risk of bleeding, ACo therapy was stopped. Six months later, TTE showed recurrent LV thrombus, and an underlying hypercoagulable state was ruled out. ACo therapy was restarted on an indefinite basis, and dual antiplatelet therapy was continued

Conclusion: This case illustrates the need for repeat ETT following the withdrawal of ACo therapy in patients with STEMI, both to monitor thrombus status and to assess LV segmental contraction.

No conflict of interest 


\title{
ACUTE SYSTEMIC CELLULAR INFLAMMATORY RESPONSE, BUT NOT CORONARY THROMBUS COMPOSITION, IS ASSOCIATED WITH IMPAIRMENT OF REPERFUSION IN STEMI PATIENTS AFTER PRIMARY PCI
}

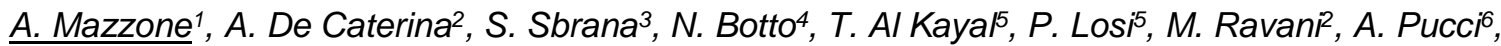 \\ S. Berti ${ }^{2}$ \\ ${ }^{1}$ Heart Hospital - Fondazione Toscana Gabriele Monasterio, Adult Cardiac /Cardiosurgery Dep., \\ Massa, Italy \\ ${ }^{2}$ Heart Hospital - Fondazione Toscana Gabriele Monasterio, Haemodinamic Laboratory, Massa, \\ Italy \\ ${ }^{3}$ Institute of Clinical Phisiology- CNR - Pisa and Massa- Italy, Biochemical Chemistry laboratory, \\ Massa, Italy \\ ${ }^{4}$ Heart Hospital - Fondazione Toscana Gabriele Monasterio, Biochemical Chemistry laboratory, \\ Massa, Italy \\ 5Institute of Clinical Phisiology- CNR - Pisa and Massa Italy, Biomaterials Laboratory, Massa, \\ Italy \\ ${ }^{6}$ Azienda Universitaria - Pisa, Pathology, Pisa, Italy
}

\begin{abstract}
Aims: No-reflow phenomenon is associated with adverse clinical outcomes in STEMI patients undergoing primary percutaneous coronary intervention (PPCI). Aims:To investigate the association between angiographic reperfusion with hystopathological features of aspirated intracoronary thrombus as well as with peripheral inflammatory and metabolic response at admission
\end{abstract}

Method: Among 119 consecutive STEMI patients undergoing primary PCI (male $76 \%$, mean age $65 \hat{A} \pm 13$ SD) in the years 2013-2014 49 thrombi were suitable for hystopathological analysis. Thrombus composition analysis was based on material organization (cell type, connective tissue and fibrin deposition) and presence of atheroma fragments. Routinely laboratory data concerning differential and total leukocyte and platelet count, glycemia, lipoproteins (HDL, LDL), GGT, CRP and fibrinogen were collected in peripheral blood samples at admission. No-reflow was defined as TIMI flow $<3$ or TIMI 3 and Myocardial Blush Grade $<2$ at the end of primary PCI.

Results: Hystopathological features of coronary thrombi did not differ according to the presence or absence of angiographic no-reflow phenomenon. Conversely, a higher number of circulating neutrophils $(p=0.03)$ and total leukocytes $(p=0.06)$ were found in patients with no reflow. Notably, neutrophils number directly correlated with acute glicemia levels $(p=0.0004)$, but not with the presence of diabetes.

Conclusion: Our data support the hypothesis that impaired myocardial reperfusion after primary $\mathrm{PCl}$ is not influenced by thrombus composition. Conversely, the extent of systemic acute inflammatory response as well as hyperglycemia might play a role, or be a marker, of no-reflow phenomenon in STEMI.

No conflict of interest 
ACS, ACUTE MYOCARDIAL INFARCTION

\title{
PREDICTORS AND IMPACT OF CONTRAST-INDUCED ACUTE KIDNEY INJURY ON 5-YEAR
} MORTALITY IN STEMI PATIENTS UNDERGOING PRIMARY PCI

\author{
V. Pavlovic ${ }^{1}$, D. Milasinovic ${ }^{1}$, J. Dobras ${ }^{1}$, Z. Mehmedbegovic ${ }^{1}$, V. Vukcevic ${ }^{1}$, M. Asanin ${ }^{2}$, \\ A. Ristic ${ }^{1}$, V. Kanjuh ${ }^{3}$, G. Stankovic ${ }^{1}$ \\ ${ }^{1}$ Clinical Center of Serbia, Department of Cardiology, Belgrade, Serbia \\ ${ }^{2}$ Clinical Center of Serbia, Emergency Department- Department of Cardiology, Belgrade, Serbia \\ ${ }^{3}$ Serbian Academy of Sciences and Arts, Board on Cardiovascular Pathology, Belgrade, Serbia
}

Aims: Contrast-induced acute kidney injury (CI-AKI) was associated with adverse outcomes after primary percutaneous coronary intervention $(\mathrm{PCl})$. We sought to identify the predictors and assess the impact of $\mathrm{Cl}-\mathrm{AKI}$ on very long-term mortality after primary $\mathrm{PCl}$.

Method: We analyzed 1722 patients referred to primary $\mathrm{PCl}$ in a high-volume center during years 2009-2010. Cl-AKI was defined as: 1$)$ absolute $(\geq 0.5 \mathrm{mg} / \mathrm{dl})$ or relative $(\geq 25 \%)$ increase in serum creatinine, 2) absolute increase $\geq 0.3 \mathrm{mg} / \mathrm{dl}$ and 3) absolute increase $\geq 0.5 \mathrm{mg} / \mathrm{dl}$, within 72 hours of intervention.

Results: The incidence of Cl-AKI (relative or absolute creatinine elevation) was $18.1 \%(n=311)$. Multivariable regression identified female sex, Killip class $>1$, diabetes and maximum CK levels as independent predictors of $\mathrm{Cl}-\mathrm{AKI}$. Patients with $\mathrm{Cl}-\mathrm{AKI}$ had higher mortality rates compared to those without at median follow-up of 4.8 years (IQR, $4.4-5.2)(27.7 \%$ vs $17.2 \%$, respectively; HR $1.75, \mathrm{p}<0.001)$. If $\mathrm{Cl}-\mathrm{AKI}$ was defined as absolute increase in serum creatinine only, the incidence was $10.1 \%(n=174)$ for creatinine elevation $\geq 0.3 \mathrm{mg} / \mathrm{dl}$ and $3.8 \%(n=65)$ for $\geq 0.5 \mathrm{mg} / \mathrm{dl}$. Cl-AKI expressed as solely absolute creatinine elevation was also significantly associated with higher mortality rate at median follow-up (HR=2.98, $p<0.001$ for $\geq 0.3 \mathrm{mg} / \mathrm{dl}$ and $\mathrm{HR} 5.38, p<0.001$ for $\geq$ $0.5 \mathrm{mg} / \mathrm{dl})$.

Conclusion: $\mathrm{Cl}-\mathrm{AKI}$ was associated with significantly higher 5 -year mortality when defined either as relative ( $\geq 25 \%$ from the baseline value) or absolute (both $\geq 0.3$ and $\geq 0.5 \mathrm{mg} / \mathrm{dl}$ ) rise in serum creatinine.

No conflict of interest 
ACS, ACUTE MYOCARDIAL INFARCTION

\title{
THE IMPACT OF ADMISSION PULSE PRESSURE IN PATIENTS WITH FIRST ST-SEGMENT ELEVATION MYOCARDIAL INFARCTION
}

\author{
A. Separham, H. Namdar, Y. Hosseinkhah, B. Sohrabi, S. Ghaffari, N. Aslanabadi \\ Cardiovascular Research Center - Tabriz University of Medical Sciences, cardiology, Tabriz, Iran
}

Aims: Arterial Pulse pressure (PP) is a main prognostic factor in many cardiovascular disease. Data regarding its effect on ST segment elevation myocardial infarction (STEMI) is scarce and conflicting. The aim of this study was to investigate the impact of admission pulse pressure on hospital outcome in patients presenting with first STEMI and its relation with pattern of coronary artery involvement.

Method: Between March 2014 and April 2015, 103 consecutive patients with first STEMI were enrolled in this study. Patients were categorized to five groups according to admission pulse pressure: $\mathrm{PP} \leq 30 \mathrm{mmHg}, \mathrm{PP}=31-40, \mathrm{PP}=41-50, \mathrm{PP}=51-60, \mathrm{PP}>60 \mathrm{mmHg}$

Results: Mean pulse pressure (PP) was $49.84 \pm 14.34 \mathrm{mmHg}$ with no significant difference between men and women. Mean pulse pressure was lower in patients who died versus survived $(39.13 \pm 13.01 \mathrm{mmHg}$ vs $50.75 \pm 4.015 \mathrm{mmHg}, \mathrm{P}=0.027)$. In patients with three vessel coronary artery disease, mean pulse pressure was higher, but the difference was not statistically significant. Pulse pressure was significantly lower in patients with Inferior myocardial Infarction than Anterior myocardial Infarction $(39 \pm 7.41 \mathrm{mmHg}$ vs $49.11 \pm 13.65 \mathrm{mmHg}, \mathrm{P}=0.03)$. With respect to hospital complications, apart from cardiogenic shock, which was significantly higher in the Low PP group (PP $\leq 30$ ) than in the High PP group (PP>60), there was no significant difference between groups in other complications.

Conclusion: In patients with first STEMI pulse pressure was significantly lower in patients who died than survived patients. Other complications were not different between groups with low and high PP. There was a trend toward higher PP in patients with multi vessel coronary involvement.

No conflict of interest 


\title{
ALLOPURINOL IN PATIENTS WITH ACUTE ST ELEVATION MYOCARDIAL INFARCTION UNDERGOING THROMBOLYTIC THERAPY : A RANDOMIZED TRIAL
}

\author{
A. Separham ${ }^{1}$, S. Ghaffari', H. Nafafi', M. Ghojazadeh² \\ ${ }^{1}$ Cardiovascular Research Center - Tabriz University of Medical Sciences, cardiology, Tabriz, Iran \\ ${ }^{2}$ Research Development Coordination Center-Tabriz university of medical science, Physiology, \\ Tabriz, Iran
}

Aims: Recent studies have suggested that allopurinol has protective effect in ischemic reperfusion injury. In this study, we aimed to evaluate the effect of allopurinol in patients with acute ST elevation myocardial infarction (STEMI) undergoing thrombolytic therapy.

Method: This randomized clinical trial included 140 patients admitted with acute STEMI and treated with thrombolysis. Patients were randomly assigned to receive allopurinol $400 \mathrm{mg}(\mathrm{n}=70)$ or placebo( $n=70)$ immediately before thrombolytic therapy. Then, allopurinol group received allopurinol $100 \mathrm{mg}$ daily for 28 days and placebo group received placebo for the same period. ST resolution rate (STR) and in-hospital and 6 months follow-up major adverse cardiac events (MACE) were compared between groups. Summed percentage ST-resolution from the pre to the post thrombolysis ECGs across all infarct- related leads were used and stratified to two groups: $<50 \%$ or $\geq 50 \%$.

Results: Patients receiving allopurinol had significantly higher rate of $S T R>50 \%(p=0.04)$ and lower levels of peak CK ( $p=0.003)$, CKMB $(p=0.005)$ and CTnI $(p<0.001)$ compared to placebo. Also, allopurinol group had significantly lower rate of in-hospital MACE $(p=0.03)$, but there was no significant difference between groups regarding in-hospital death $(p=0.39)$ and six months mortality $(p=0.51)$.

Conclusion: In patients with acute STEMI treated with thrombolysis, allopurinol treatment was associated with better ST segment resolution, reduced enzymatic infarct size and better inhospital outcome but no effect on six month mortality was seen.

No conflict of interest 
ACS, ACUTE MYOCARDIAL INFARCTION

\title{
EXPERIMENTAL STUDY OF EVALUATING LEFT VENTRICULAR REGIONAL SYSTOLIC FUNCTION USING THREE-DIMENSIONAL SPECKLE TRACKING ECHOCARDIOGRAPHY IN ACUTE MYOCARDIAL INFARCTION MODEL OF DOGS
}

\author{
G. Song, Z. Ruifang \\ The First Affiliated Hospital of Zhengzhou University, Ultrasound, Zhengzhou, China
}

\begin{abstract}
Aims: To investigate the value of three-dimensional speckle tracking imaging (3D-STI) in assessment of left ventricular regional systolic function in acute myocardial infarction model of dogs.
\end{abstract}

Method: Left anterior descending coronary artery was ligated in 24 adult beagle dogs. Longitudinal strain (LS) of every left ventricular segment before ligation and 180min after ligation, and the time for acquisition and analysis were obtained using two-dimensional speckle tracking imaging (2D-STI) and 3D-STI. After the experiment, left ventricular myocardium were determined by TTC staining and divided into two groups: infarcted myocardial segments and non-infarcted myocardial segments.

Results: Experimental model of acute myocardial infarction was established successfully in 20 dogs. After ligation, both LS of 2D-STI and 3D-STI in infarcted myocardial segments were significantly lower $(P<0.01)$. The time for acquisition and analysis of the images by 3D-STI were significantly shorter than that by 2D-STI $(P<0.01)$. The area under the ROC curve of 3D-STI was larger than that of 2D-STI. A cut-off value of $-12.56 \%$ for LS of the 3D-STI had $77.9 \%$ sensitivity and $88.2 \%$ specificity in predicting acute myocardial infarction.

Conclusion: 3D-STI is a convenient, accurate method for evaluating left ventricular regional systolic function.

No conflict of interest 
ACS, ACUTE MYOCARDIAL INFARCTION

\section{COMPARISON OF CARES IN PATIENT WITH ACUTE MYOCARDIAL INFARCTION HOSPITAL IN PREHOSPITAL WITH CURRENT GUIDELINES}

M. Zarei ${ }^{1}$, M. Dastras ${ }^{2}$

${ }^{1}$ North Khorasan University of Medical Sciences - Bojnurd - Iran, Shirvan, Iran

'Zahedan University of Medical Sciences- Zahedan- Iran, Nursing, Zahedan, Iran

Aims: Some progress has been resulted in the treatment of acute myocardial infarction; led to significant improvement of treatment result. Increased use of pre-hospital medicine emergency, cardiac intensive cares, defibrillation, administration of beta-blockers and thrombolytic therapy, aspirin and therapy interventional procedures has been caused to reduce mortality. This study was done aimed to evaluate the care of patients with angina and comparison to standard.

Method: In this descriptive-comparative study, 300 pre-hospital processes medical emergency center in Zahedan were selected and evaluated by sampling method. Conducted to evaluate the efficacy of each of the pre-hospital care, according to the standard procedure of extraction and on standard of state emergency the planed checklist and information were collected by interview and observation producers. Evaluation of patient care was performed from the moment of patient arrival to ambulance to admission in the Imam Ali hospital in Zahedan.

Results: Mean patient transfer time is 31.74 minutes compared to the standard (35 minutes). $95 \%$ of respondents had participated in the specialized course of heart and there is significant relationship between of the compliance measures of care of patients with acute myocardial infarction from arrival moment to ambulance to the adoption in Imam Ali hospital in Zahedan and passing emergency specialized courses of heart.

Conclusion: Care performances don't consistent $91.3 \%$ with standard and $8.7 \%$ have not met the standards.

No conflict of interest 


\title{
EPIDEMIOLOGY, PREVENTION, RISK FACTORS
}

\section{QUALITY OF LIFE IMPROVEMENT AFTER A CARDIAC REHABILITATION PROGRAM IN HIGH-RISK PATIENTS}

\author{
E. Alegria-Barrero ${ }^{1,2}$, M.A. Blazquez ${ }^{1}$, C. Sánchez-Rodríguez ${ }^{1}$, M. Ordoñez ${ }^{3}$, H. Diaz ${ }^{4}$, \\ J.M. Barahona ${ }^{5}$, S. Trinidad ${ }^{6}$, S. Alonso-Viteri ${ }^{7}$, M.A. San Martin ${ }^{1}$ \\ ${ }^{1}$ Hospital Universitario de Torrejon, Cardiology, Madrid, Spain \\ ${ }^{2}$ Universidad Francisco de Vitoria, Medicine, Madrid, Spain \\ ${ }^{3}$ Hospital Universitario Fundación Jiménez Díaz, Cardiology, Madrid, Spain \\ ${ }^{4}$ Hospital Universitario de Torrejon, Psychiatry, Madrid, Spain \\ ${ }^{5}$ Hospital Universitario de Torrejon, Psychology, Madrid, Spain \\ ${ }^{6}$ Hospital Universitario de Torrejon, Rehabilitation and Physical Medicine, Madrid, Spain \\ ${ }^{7}$ Hospital Universitario de Torrejon, Neumology, Madrid, Spain
}

Aims: To investigate quality of life improvement among cardiovascular high-risk patients after a cardiac rehabilitation program.

Method: From 2013 to 2015, 153 patients were included in a hospital-based cardiac rehabilitation program (CRP). Median duration of the program was 8 weeks. Increasing phyisical exercise was programmed according to an exercise test, based on maximal heart rate achieved. All patients trained with continuous EKG monitoring. Quality of life was assessed using the SF-36 questionnaire before and after completing the program. Exercise test was performed in all patients before and after the program and metabolic equivalents (METS) were measured. Finally, New York Heart Association (NYHA) functional class was measured before and after the program.

Results: $75.2 \%$ patients were male, median age 57 (IQR 14). $87 \%$ of the patients had a previous history of ischemic heart disease. Mean ejection fraction was 0.57 . Exercise capacity boosted significantly after the program (6.9 METS vs 7.99 METS, $p<0.001)$. Significantly, quality of life scores increased significantly at the end of the program (62.2\% vs $73.7 \%, p<0.001)$. Accordingly, NYHA functional class significantly improved during the program:


At the beginning of the program, $46.4 \%$ were in NYHA class I. After 8 weeks of CRP, $79.7 \%$ were in NYHA class I. In addition, a significant reduction of body mass index was achieved (29.6 vs 28.8, $\mathrm{p}<0.001$ ). 
Conclusion: Cardiac rehabilitation significantly improved quality of life, exercise capacity and functional class in cardiac high-risk patients.

No conflict of interest 
295

EPIDEMIOLOGY, PREVENTION, RISK FACTORS INCIDENCE OF SINGLE CORONARY ARTERY WITH ABSENCE OF LEFT MAIN CORONARY
ARTERY-CASE REPORT AND FOLLOW UP OF SEVEN AND FOURTEEN YEARS

M. De Oliveira

Rio De Janeiro, Brazil

Aims: The coronary arteries has many anatomical variations. The incidence of right coronary artery issuing branch for the circumflex artery is

reported in medical literature. However the agenesis of Left Main Coronary Artery and RCA giving the same branch to the Cincunflex Artery and Left Anterior Descending Artery is a rare anatomic variant which leads to great vulnerability when such patients are suffering from ischemic heart disease.

Method: We prospectively studied two patients, one male aged 60 years and one female with 80 years with complaints of atypical chest pain.Both the echocardiogram was normal but the test was positive electrocardiographically. Both underwent coronary angiography that showed a dominant RCA filling the Left Anterior Descending Coronary Artery and Circumflex Coronary Artery and agenesis of Left Main Coronary Artery.Both were informed, enlightened under its pathology and instructed to perform strict lipid-lowering diet, regular moderate physical activity, antiplatelet use (AAS500mg),20 mg of simvastatin evening and rigorous clinical control every six months.

Results: Despite this anatomical variation is extremely rare leading to greater vulnerability to ischemic heart disease both are free of symptoms

due to a judicious control of risk factors. The male patient for 7 years and female 14 years. When this happens we have a single coronary artery to the vasculature of the heart.

Conclusion: The existence of the RCA with agenesis of LMCA is an anatomical variation that causes the extremely vulnerable patient when suffering from ischemic heart disease due to inability to develop side branches. Both reported cases the strict control of risk factors alowed them to lead a normal life until now.

No conflict of interest 


\title{
296 \\ EPIDEMIOLOGY, PREVENTION, RISK FACTORS \\ THE GROWTH DIFFERENTIATION FACTOR 15, A NEW PROGNOSTIC MARKER IN DIABETIC CARDIOMYOPATHY
}

\author{
$\underline{P .}$ Avanzas $^{1}$, A. Dominguez-Rodriguez ${ }^{2}$, J. Gonzalez-Gonzalez², C. Belleyo-Belkasem², \\ P. Abreu ${ }^{3}$ \\ ${ }^{1}$ Hospital Universitario Central de Asturias, Cardiology, Oviedo, Spain \\ ${ }^{2}$ Hospital Universitario de Canarias, Cardiology, Santa Cruz de Tenerife, Spain \\ 3Universidad de La Laguna, Departamento de Ciencias Médicas Básicas Unidad de Fisiología, \\ Santa Cruz de Tenerife, Spain
}

Aims: Diabetic cardiomyopathy (DC) presents asymptomatic in early stages. The growth differentiation factor-15 (GDF-15) is a cytokine secreted by cardiomyocytes in response to oxidative stress and inflammation. The aim was to assess the prognostic value of GDF-15 levels in patients with DC.

Method: Pilot prospective study that included 213 asymptomatic diabetic patients. For the purpose of the analysis we analysed data of 45 patients with DC (ESC criteria). Patients with DC were divided in two groups, according to the presence or absence of the primary endpoint: heart failure and/or angina with hospitalization during 1 year follow up period.

Results: The primary endpoint presented in 12 patients (26.7\%). There were no statistically significant differences in baseline variables between the two groups. GDF-15 levels were higher in patients who presented events (6458.9 [5359.7 to 8681.9] vs 4706 [3719-6463] pg/ml, $\mathrm{p}=0.007$ ) (Figure). Cox survival analysis showed, after adjusting for other covariates, that the median GDF-15 levels (HR $=4.96,95 \% \mathrm{Cl}[1.24$ to 19.77]; $p=0.023)$ and LVEF (HR= $0.83,95 \%$ $\mathrm{Cl}$ [0.7-0.98]; $p=0.031$ ) were independent predictors of the combined endpoint. Model C statistic was 0.826 .

Conclusion: GDF 15 levels predict outcome in patients with DC. Randomized studies are needed with larger sample sizes in order to add more information regarding the predictive value of this biomarker.

No conflict of interest 
297

\title{
EPIDEMIOLOGY, PREVENTION, RISK FACTORS
}

\section{TEN YEAR SURVIVAL OF THREE-VESSEL CORONARY DISEASE PATIENTS IN MIRROR OF RETROSPECTIVE SYNTAX SCORE CALCULATION}

\author{
L. Balogh ${ }^{1}$, Z. Bereczky², É. Katona ${ }^{2}$, G. Kolodzey ${ }^{1}$, Á. Mandzák ${ }^{1}$, I. Czuriga ${ }^{1}$ \\ ${ }^{1}$ University of Debrecen - Faculty of Medicine, Cardiology and Cardiac Surgery, Debrecen, \\ Hungary \\ ${ }^{2}$ University of Debrecen - Faculty of Medicine, Division of Clinical Laboratory Science, Debrecen, \\ Hungary
}

Aims: The aim of the study was retrospective validation of Syntax score by determination of survival among three-vessel disease patients.

Method: 955 consecutive patients who underwent cardiac catheterization were screened for the study in a 18-month period. Among them 611 patients had significant $(\geq 50 \%)$ coronary stenosis in a major coronary artery; 212 of them had three vessels affected. Upon enrollment serum lipid parameters, homocysteine, folic acid, vitamin B12, C-reactive protein and fibrinogen were determined by routine laboratory methods. Presence of diabetes mellitus, hypertension and smoking habits were recorded and ejection fraction was calculated.

Results: Among these patients 131 individuals had myocardial infarction in their case histories, and at recruitment 113 patients underwent coronary bypass grafting and 59 patients had coronary stent (BMS) implantation. After 10 years of follow up Syntax score was calculated retrospectively based on the coronarography images and myocardial death as end-point was recorded. Survival time showed significant negative correlation with diabetes mellitus, myocardial infarction in the case history, C-reactive protein and syntax score, while significant positive correlation with ejection fraction value that was recorded upon enrollment. Primer end-point (myocardial death, $n=77)$ was significantly influenced by the presence of diabetes mellitus $(p=0.036)$, age $(p=0.005)$ and ejection fraction $(p=0.001)$ at enrollment. The other examined laboratory parameters were not predictive for survival.

Conclusion: Mean Syntax score was 31.49 in CABG and 22.7 in PCl group, respectively. The heart team decision is mainly influenced by anatomical complexity of the lesions and retrospectively calculated Syntax score values were similar to guidelines used now for decision making.

No conflict of interest 


\title{
EPIDEMIOLOGY, PREVENTION, RISK FACTORS
}

\section{RESIDUAL BURDEN OF DYSLIPIDEMIA IN THE US AND POTENTIAL SOCIAL VALUE OF PCSK9 INHIBITORS IN HIGH RISK POPULATIONS}

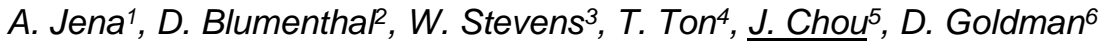 \\ ${ }^{1}$ Harvard Medical School, Health Care Policy, Boston, USA \\ ${ }^{2}$ Massachusetts General Hospital, Internal Medicine, Boston, USA \\ ${ }^{3}$ Precision Health Economics, Health Economics, Los Angeles, USA \\ ${ }^{4}$ Precision Health Economics, Health Analytics, Los Angeles, USA \\ ${ }^{5}$ Precision Health Economics, Health Services Research, Los Angeles, USA \\ ${ }^{6}$ University of Southern California, Schaeffer Center, Los Angeles, USA
}

\begin{abstract}
Aims: To estimate the residual burden of dyslipidemia in the U.S. among patients with atherosclerotic cardiovascular disease, familial hyperlipidemia/ hypercholesterolemia, and diabetes and the potential social economic value of reducing LDL cholesterol (LDL-C) by $50 \%$ for those not at-goal $(<70 \mathrm{mg} / \mathrm{dL})$ on lipid-lowering therapy (LLT). The economic value of PCSK9 inhibitors (PCSK9i) in high risk groups will be estimated.
\end{abstract}

Method: The study population was projected for 2015-2035 using the National Health and Nutrition Examination Survey and the U.S. Census. For those not at LDL-C goal, we estimated incidence rates for major adverse cardiovascular disease (MACE) events with a large insurance claims dataset and mortality rates with vital statistics data. Using evidence on the association between LDL-C and MACE, we estimated the value to the U.S. of reducing LDL-C levels by $50 \%$ in all risk groups. We incorporated results from meta-analysis of PCSK9i to estimate the economic value of PCSK9i among high risk patients receiving standard LLT but not at-goal.

Results: Among patients on status quo LLT, MACEs will rise from 1.7 million in 2015 to 2.3 million in 2035. Novel LLTs that may reduce LDL-C levels or MACE events by $50 \%$, such as PCSK9i, could cumulatively avert 15-21 million MACEs and .7-1.3 million cardiovascular deaths by 2035 . The potential social value from averted deaths and hospitalizations due to improved lipid control was estimated between \$1.3-2.5 trillion.

Conclusion: The projected residual burden of dyslipidemia over the next 20 years is large. For certain high-risk groups, PCSK9i could create considerable clinical and social value.

Conflict of Interest Disclosure Statement:

This research was sponsored by Amgen. Anupam Jena is a principal consultant at, Daniel Blumenthal is a consultant at, and Dana Goldman is a partner of Precision Health Economics, a Precision for Medicine company, a health economics consulting firm that provides services to the life sciences industry. 


\title{
EPIDEMIOLOGY, PREVENTION, RISK FACTORS
}

\section{DIFFERENT PREDICTIVE VALUES OF LDL- AND HDL- CHOLESTEROL FOR CARDIOVASCULAR AND CEREBROVASCULAR DISEASE IN THE GENERAL JAPANESE POPULATION}

\author{
S. Konno, M. Munakata \\ Tohoku Rosai Hospital, Division of Hypertension, Sendai, Japan
}

Aims: We have recently reported that moderately increased albuminuria (formerly known as microalbuminuria) and high-density lipoprotein (HDL) cholesterol are independent risk factors for the composite of cardio/cerebrovascular events (stroke, acute myocardial infarction and coronary revascularization) in the general Japanese population. However, the differential association of lipid risk factors with cerebrovascular and cardiovascular disease, respectively, in this population has not been fully elucidated. The aim of this study was to compare the predictive values of lipid markers for cerebrovascular and cardiovascular disease in the general Japanese population.

Method: A total of 3093 inhabitants of Watari town, Miyagi prefecture, Japan, who participated in an annual health check-up in 2009 was enrolled in this prospective cohort study. Anthropometry, sitting blood pressures, fasting blood samples for lipid/glucose measurements, and spot urine albumin-to-creatinine ratio were examined. Participants were followed prospectively for up to 68 months.

Results: During a mean follow-up period of 52.6 months, 52 stroke events and 24 coronary heart disease events (acute myocardial infarction and coronary revascularization) were observed. Multivariate Cox proportional hazards analysis demonstrated that low-density lipoprotein (LDL) cholesterol was an independent risk factor for coronary heart disease events (HR: 1.022 per $1 \mathrm{mg} / \mathrm{mL}$ increase, $95 \% \mathrm{Cl}: 1.011-1.033$ ), while HDL (but not LDL) cholesterol was an independent risk for stroke events (HR: 0.976 per $1 \mathrm{mg} / \mathrm{mL}$ increase, $95 \% \mathrm{Cl}: 0.958-0.999)$ after adjustment for possible confounding factors.

Conclusion: The predictive values of LDL and HDL cholesterol could be different for cardiovascular and cerebrovascular events in the general Japanese population.

No conflict of interest 
300

EPIDEMIOLOGY, PREVENTION, RISK FACTORS

\title{
INFLUENCE OF GENETIC RISK FACTORS ON CORONARY HEART DISEASE OCCURRENCE IN AFRO-CARIBBEANS
}

\author{
L. Larifla ${ }^{1,2,3}$, K. Beaney ${ }^{1}$, L. Foucan ${ }^{3}$, J. Bangou ${ }^{3}$, C.T. Michel${ }^{2}$, J. Cooper ${ }^{1}$, S.E. Humphries ${ }^{1}$ \\ ${ }^{1}$ University College London, Centre for cardiovascular genetics, London, United Kingdom \\ ${ }^{2}$ University Hospital of Pointe-à-Pitre, Department of cardiology, Pointe-à-Pitre, France \\ ${ }^{3}$ University of Antilles, Research Group Clinical Epidemiology and Medicine- \\ ECM/L.A.M.I.A EA 4540, Pointe-à-Pitre, France
}

Aims: Despite excessive rates of cardiovascular risk factors such as hypertension, diabetes and obesity, Afro-Caribbeans have lower mortality rates from coronary heart disease (CHD) compared to Caucasians. This study evaluated the association of genetic risk markers previously identified in Caucasians, with CHD in Afro-Caribbeans.

Method: We studied 537 Afro-Caribbeans subjects (178 cases with documented CHD and 359 controls) who were genotyped for $19 \mathrm{CHD}$-related SNPs. A genetic risk score (GRS) incorporating the 19 SNPs was calculated. These participants were compared to 1360 Caucasian subjects from the_second Northwick Park Heart Study (NPHS-II).

Results: In Afro-Caribbeans, patients with $\mathrm{CHD}$ had higher rates of hypertension $(78.7 \%$ vs $30.1 \%$ ), hypercholesterolemia ( $52.8 \%$ vs $15.0 \%$ ), diabetes $(53.9 \%$ vs $14.8 \%)$, and were more often male $(64.0 \%$ vs $43.7 \%$ ) and smokers (27.5\% vs $13.4 \%$ ) compared to non-CHD controls (all $\mathrm{p}<0.001)$. The GRS was higher in Afro-Caribbeans with CHD compared to those without CHD (13.90 vs $13.17 ; p<0.001)$ and was significantly associated with $\mathrm{CHD}$ after adjustment for cardiovascular risk factors with an odds ratio of $1.40(95 \% \mathrm{Cl} 1.09-1.80)$ per standard deviation unit. There were significant differences in allelic distributions between the two ethnic groups, for 14 of the 19 SNPs. The GRS was substantially lower in Afro-Caribbean controls compared to Caucasian controls: 13.17 vs $16.59 ; p<0.001$

Conclusion: This study demonstrates that a multilocus-GRS composed of 19 SNPs associated with CHD in Caucasians is a strong predictor of the disease in Afro-Caribbeans. The differences in CHD mortality between Afro-Caribbeans and Caucasians might be a result of significant discrepancies in common gene variant distribution.

No conflict of interest 
301

\title{
EPIDEMIOLOGY, PREVENTION, RISK FACTORS
}

\section{FRAMINGHAM RISK SCORE FOR PREDICTION OF FUTURE MORTALITY IN PATIENTS TREATED WITH PRIMARY PCI FOR ACUTE CORONARY SYNDROME}

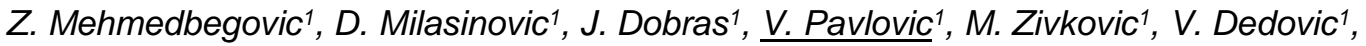 \\ V. Vukcevic ${ }^{1}$, V. Kanjuh ${ }^{2}$, G. Stankovic ${ }^{1}$ \\ ${ }^{1}$ Clinical Center of Serbia, Cardiology Clinic, Belgrade, Serbia \\ ${ }^{2}$ Serbian Academy of Sciences and Arts, Board on Cardiovascular Pathology, Belgrade, Serbia
}

Aims: Framingham risk score (FRS) is most widely accepted cardiovascular (CV) risk stratification system predicting probability of CV event in gender specific manner. Usefulness of score for prediction of future events after primary CV event occurred is less well established.

Method: In a period from 2009-2010 we identified 2206 consecutive patients less than 80 years of age treated with primary $\mathrm{PCl}$ for acute coronary syndrome. Patients with previous history of ischemic coronary event (myocardial infarction or PCl; 807 pts) and those that died during initial hospitalization period (94 pts) were excluded. Final FRS was calculated for 1230 (94\%) pts.

Results: Mean age of the selected population was $58,3 \pm 10,6$ and most of them were male 870 $(70,7 \%)$. Mean FRS for men was 14,3 $\pm 3,4$ and for women 17,8 $\pm 3,0$. Long-term FU was performed in 1140 pts (93\%) with mean duration of $51,8 \pm 16,5$ months, during which 139 pts $(12,2 \%)$ died. Higher value of FRS was able to predict future mortality only in population of men $(15,2 \pm 3,4$ vs $14,2 \pm 3,2 ; p=0.008)$ while in women such interaction was not observed $(18,0 \pm 2,9$ vs $17,8 \pm 3,1 ; p=0.718)$.

Conclusion: Framingham risk score after initial cardiovascular event is good predictor of very long-term mortality in men and not in women. Other factors in addition to FRS component should be evaluated in future studies to predict mortality risk in female patients.

No conflict of interest 
302

\title{
EPIDEMIOLOGY, PREVENTION, RISK FACTORS
}

\section{THE INFLUENCE OF BODY MASS INDEX AND METABOLIC RISK VARIABLES ON PULSE WAVE VELOCITY IN HEALTHY POPULATION}

\author{
M.I. Mendonca ${ }^{1}$, A. Pereira ${ }^{1}$, R. Rodrigues ${ }^{1}$, A.I. Freitas $^{1}$, M. Neto ${ }^{1}$, A.C. Sousa ${ }^{1}$, E. Henriques ${ }^{1}$, \\ C. Freitas ${ }^{1}$, R. Palma dos Reis ${ }^{2}$ \\ ${ }^{1}$ Funchal Hospital Centre, Research Unit, Funchal, Portugal \\ ${ }^{2}$ New University of Lisbon, Cardiology, Funchal, Portugal
}

Aims: Measurement of femoral-carotid pulse wave velocity (PWV) is recognized as a simple and practical method for assessing arterial stiffness. We determined whether the PWV of asymtomatic population with no detectable coronary artery disease is affected by obesity and its associated metabolic risk variables.

Method: A cross-sectional sample of 1231 controls (26.5\% female and $73.5 \%$ male), aged $30-65$ years, were recruited for this study. PWV was measured by a simple automatic oscillometric technique. Adiposity measures, blood pressure, serum lipoproteins, fasting glucose were obtained.

Results: The PWV of the controls was significantly higher in men $(8.4 \pm 1.8 \mathrm{~m} / \mathrm{s})$ than in women $(7.4 \pm 1.6 \mathrm{~m} / \mathrm{s}, \mathrm{p}<0.0001$ and increased with age in both genders specially in the female population (male $r=0.504, p<0.0001$, female $r=0.689 p<0.0001$ ). After being statistically adjusted for age and gender, PWV was significantly correlated with waist-to-height ratio $(r=0.235 p<0.0001)$, systolic and diastolic blood pressures $(r=0.576 p<0.0001)$, mean arterial pressure $(r=0.569 p<0.0001)$ and fast glicose $(r=0.217)$. Low correlation with triglycerides, BMI (Body Mass Index),

Atherogenicíndex of Plasma were found. In the multivariate regression analysis, BMI, systolic and diastolic blood pressure and age were found to be significant determinants of PWV $(P<0.001)$. An increasing number of clustered risk variables, including high values $(75 \%$ quartiles) of Systolic and Dyastolic Pressure, BMI, waist-to-height ratio, and atherogenic index showed a graded association with PWV.

Conclusion: These results suggest that obesity and its associated metabolic abnormalities are important factors in the increased PWV of healthy population and that may be useful in investigating early arterial wall changes in this population.

No conflict of interest 


\title{
RISING PREVALENCE OF CORONARY HEART DISEASE IN VELLORE, TAMIL NADU, SOUTH INDIA BETWEEN 1991-94 TO 2010-12: A REPEAT CROSS SECTIONAL STUDY
}

\author{
A. Oommen 1 , V. Abraham ${ }^{1}$, K. George ${ }^{1}$, J.V. Jose 2 \\ ${ }^{1}$ Christian Medical College, Community Health, Vellore, India \\ ${ }^{2}$ Christian Medical College, Cardiology, Vellore, India
}

Aims: With the increase of cardiovascular risk factors in India, the prevalence of coronary heart disease is also expected to rise. A cross sectional study in 2010-12 assessed the prevalence of coronary heart disease in Vellore, Tamil Nadu and compared the current prevalence with the prevalence of coronary heart disease in the same areas in 1991-94.

Method: A cross sectional survey was carried out as part of a WHO STEPS survey for noncommunicable diseases which also assessed the prevalence of coronary heart disease (defined as previously diagnosed disease, symptoms detected using Rose angina questionnaire or ischemic changes on electrocardiography) in a rural block in Vellore district and in Vellore town, south India, in 2010-12. A similar survey had been carried out in the same rural and urban area in 1991-94. The numbers of participants were 7342 in 1991-94 and 4845 in 2010-12, aged 30 to 60 years.

Results: The age adjusted prevalence of coronary heart disease in rural and urban women doubled from $24 / 1000$ to $63 / 1000$ and $55 / 1000$ to $127 / 1000$ respectively, with only a slight increase among males, between 1991-94 and 2010-12 in both urban and rural Vellore (Table).

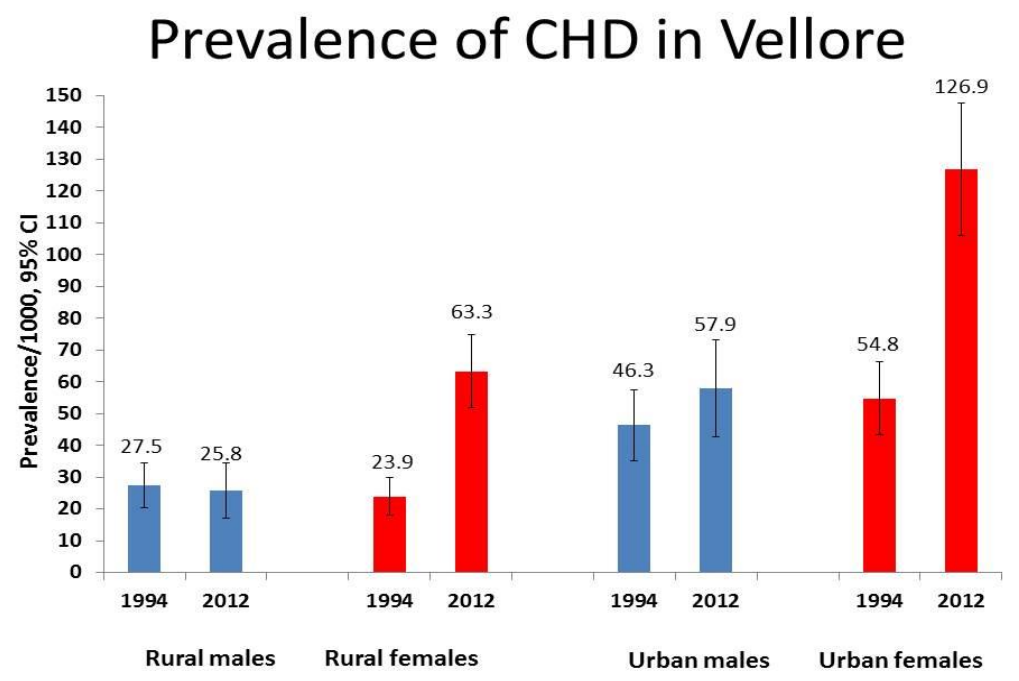

Conclusion: The large increase in prevalence rates of coronary heart disease among both rural and urban women in India, suggests the need for further confirmatory studies and interventions for prevention, especially targeting women who are generally considered to be at a lower risk for coronary heart disease. 
No conflict of interest 
304

EPIDEMIOLOGY, PREVENTION, RISK FACTORS

COGNITIVE FUNCTION AND DEPRESSIVE SYMPTOMS IN PATIENTS WITH CORONARY ARTERY DISEASE: ANSAN GERIATRIC COHORT STUDY

M.H. Park ${ }^{1}$, J.Y. Min ${ }^{2}$

${ }^{1}$ Korea University Medical Center, Neurology, Ansan, Korea

${ }^{2}$ Fiona OB\&GY Clinic, OB\&GY, Seoul, Korea

Aims: The aim of the present study was to examine the association between coronary artery disease and cognitive function in the elderly Koreans.

Method: We recruited 601 elderly Koreans (mean age, 72.52 years; $40.8 \%$ men) from Ansan Geriatric Cohort Study (AGE study). Cognitive function was assessed by Mini-Mental State Examination (MMSE) and Montreal Cognitive Assessment (MoCA). Depressive symptoms was assessed by Geriatric Depression Scale (GDS). Coronary artery disease or cardiovascular risk factors were retrospectively ascertained with medical record.

Results: A total of 91 (15.1\%) elderly were reported to have coronary artery disease. There was no statistical difference in demographic data (age, gender, and educational level) between the presence or absence of coronary artery disease. The elderly with coronary artery disease had lower cognitive function than those without (24.61 vs. 25.34 in MMSE, $p<0.001 ; 19.61$ vs. 20.73 in MoCA, $p=0.023)$. There is no statistical difference in depressive symptoms between the presence or absence of coronary artery disease (7.31 vs. 6.91 in GDS, $p>0.05$ ).

Conclusion: Cognitive function is associated with coronary artery disease in elderly Koreans, suggesting that coronary artery disease is a potential risk factor for cognitive impairment in elderly people.

No conflict of interest 
305

EPIDEMIOLOGY, PREVENTION, RISK FACTORS

\title{
CORONARY HEART DISEASE, ATHEROSCLEROSIS AND ASSOCIATION WITH IMMUNOSUPPRESSIVE TREATMENT IN PATIENTS WITH RHEUMATOID ARTHRITIS
}

\author{
M. Parvu ${ }^{1}$, S. Coman ${ }^{2}$, A. Burlui ${ }^{3}$, E. Rezus ${ }^{4}$ \\ ${ }^{1}$ Emergency County Hospital Tirgu Mures- University of Medicine and Pharmacy Tirgu Mures, \\ Rheumaology, Tirgu Mures, Romania \\ ${ }^{2}$ Emergency County Hospital Tirgu.Mures, Cardiology, Tirgu Mures, Romania \\ 3University of Medicine and Pharmacy "Grigore T. Popa"- lasi- Clinical Rehabilitation Hospital- \\ lasi, Rheumatology- Rehabilitation, lasi, Romania \\ 4University of Medicine and Pharmacy "Grigore T. Popa"- Iasi- Clinical Rehabilitation Hospital- \\ Iasi- Romania, Rheumatology- Rehabilitation, Iasi, Romania
}

\begin{abstract}
Aims: The major contributing factor to increased cardiovascular risk in patients with rheumatoid arthritis (RA) is premature and accelerated atherosclerosis, a common complication of autoimmune diseases. The drugs used in the treatment of RA can be useful in reducing the cardiovascular risk, and subclinical atherosclerosis.
\end{abstract}

The purpose of the study was to establish the incidence of coronary heart disease in RA, and assess the role of a treatment with atheroprotective feature.

Method: We performed a retrospective study which included 113 patients diagnosed with RA, ACR/EULAR 2010 criteria. Patients were evaluated clinically and serologically, calculated Framingham cardiovascular risk score (SF), subclinical atherosclerosis was assessed by measuring IMT and atherosclerotic plaques evaluated at this level. The carotid intimae-media thickness was measured at the site of the common carotid artery using B-mode ultrasound. Coronary heart disease (CHD) was evaluated at ECG recorded and medical chart.

Results: $17 \%$ of patients were male and $83 \%$ were female, mean age $53,2 \pm 11,2$ SD, with $6,28 \pm 5,04 \mathrm{SD}$ mean duration of diseases. $55 \%$ of patients have current or former treatment with methotrexate (MTX), and $45 \%$ treated with other diseases modifying antirheumatic drugs (DMARDs) and/or NSAID. 48\% of patients presented signs or symptoms of CHD. IMT was mean value $0,95 \mathrm{~mm} \pm 0,26 \mathrm{SD}$ at male and $0,82 \mathrm{~mm} \pm 0,17 \mathrm{SD}$ at female. Patients treated with MTX had lower IMT $p=0.03$, compared to those treated with other DMARDs $p=0.45$. SF was associated with atherosclerotic plaques with $\mathrm{p}-0,0001$.

Conclusion: Our data suggest that MTX has an atheroprotective role compared to other DMARDs therapies, CHD is less frequent at these patients.

No conflict of interest 
306

EPIDEMIOLOGY, PREVENTION, RISK FACTORS

\title{
SITAGLIPTIN CAN REDUCE THE INCIDENCE OF HYPOGLYCAEMIC EPISODES IN DM2 PATIENTS HOLDING DRIVER'S LICENCE: DATA FROM EVERYDAY PRACTICE
}

\author{
K. Vukelič́ ${ }^{1}$, A. Juretič², B. Rogelj ${ }^{3}$ \\ ${ }^{1}$ Health centre Grosuplje, Diabetes, Grosuplje, Slovenia \\ ${ }^{2}$ Faculty of medicine, Internal medicine, Ljubljana, Slovenia \\ ${ }^{3}$ University of Ljubljana, Statistics, Ljubljana, Slovenia
}

\begin{abstract}
Aims: Hypoglycemia is a risk factor for life-threatening events in diabetic patients, particularly if elderly or with CAD. Tight glycemic control may increase the risk of hypoglycemia, which can be particularly dangerous if occurring while driving a car. We interviewed patients about hypoglycemic episodes with validated questionnaire, during therapy with sulfonylurea or after switch to sitagliptin.
\end{abstract}

Method: Patient files were reviewed, searching for diabetics holding driver's licence and treated with Sulfonylurea. Those reporting hypoglycemic episodes $(n=30)$ were switched to sitagliptin. Incidence of hypoglycemic episodes was compared before and after therapy switch.

Results: Of 30 diabetic patients $(62,37 \pm 8,88$ years old, $60 \%$ males) $17 \%$ had CAD. During sulfonylurea treatment, $30 \%$ of them reported $\geq 1$ hypoglycemic episode with symptoms over 3 months (which was severe in $13.3 \%$ of patients); in contrast, only $3,3 \%$ of those patients had symptoms after switching to sitagliptin.. Patients treated with sulfonylurea had significantly more symptoms of hypoglycemia $(t=6,496 p<0,001)$, more measured hypoglycemia $(t=3,523 p=0,001)$ and severe hypoglycemia $(\mathrm{t}=2,112 \mathrm{p}=0,043)$. Body weight was significantly higher in sulfonylurea group $88,36 \pm 17,38$ vs. In sitagliptin group $86,93 \pm 16,86$ after follow up.

Conclusion: Effective control of hypoglycemia is an important goal in diabetics. In addition to pathophysiological reasons, there are also practical considerations to prevent hypoglycemia as in drivers to prevent car accidents. We showed that therapy switch to sitagliptin resulted in a significant decrease of hypoglycemic episodes. A statistically significant decrease in body weight was also observed. Sitagliptin is a safer approach in »real world patients".

No conflict of interest 
307

\section{EPIDEMIOLOGY, PREVENTION, RISK FACTORS}

\section{CLINICAL IMPACT HETEROGENEITY OF GWAS CANDIDATE GENES FOR CARDIOVASCULAR DISEASES IN ETHNIC DIFFERENT POPULATIONS}

$\underline{Y . X i e}$

St. John's, Canada

Aims: Genome-wide association studies (GWAS) have accomplished significant achievements in the study of human complex diseases including cardiovascular diseases (CVD). However, many of the candidate disease genes identified by GWAS fail to be confirmed in studies using different ethnic populations, which indicate existence of a variety of genetic modifiers among different populations.

Method: DNA from 4620 patients and controls was collected including a) 1000 myocardial infarction (MI) patients and 1000 controls from the Newfoundland population; and b) 1,429 ischemic stroke patients and 1,191 controls from North Chinese Han population. Sequenom's MassARRAYÔ system was used to genotype the 15 variants from 12 selected prior GWAS candidate genes/loci in a screening population (1,000 MI patients and controls). The positive associated gene(s) were further validated by genotyping remainder patients and controls by using real-time PCR. Statistical power was calculated using QUANTO V1.2.3 software.

Results: Among the 12 studied genes/loci, only the association of 9p21 locus was successfully replicated in the Newfoundland population. The 9p21 locus was determined within an interval from rs 133049 to rs238307 (44KB) which encompasses three conjunctional LD blocks. The same locus was subsequently genotyped in Chinese patients and controls, and the association was confirmed for the rs2383207 SNP only, but not for the other three SNPs.

Conclusion: 1) the chromosome 9p21 locus is confirmed to be a genetic susceptibility locus for CVD in both Newfoundland and Northern Chinese Han populations; 2) the critical region of 9p21 locus could be as small as an interval with size less than $28 \mathrm{~kb}$.

No conflict of interest 


\title{
HYPERTENSION
}

\section{MANAGEMENT OF RESISTANT HYPERTENSION IN A RESISTANT HYPERTENSION UNIT}

\author{
E. Alegria-Barrero ${ }^{1,2}$, S. Alonso-Viteri ${ }^{3}$, R. Teijeiro-Mestre ${ }^{1}$, J. Ruiz-Garcia' ${ }^{1,2}$, E. Besada ${ }^{4}$, \\ A.I. Franco ${ }^{5}$, M.A. Blazquez ${ }^{1}$, M. Vega ${ }^{1}$, M.A. San Martin ${ }^{1}$ \\ ${ }^{1}$ Hospital Universitario de Torrejon, Cardiology, Madrid, Spain \\ ${ }^{2}$ Universidad Francisco de Vitoria, Medicine, Madrid, Spain \\ ${ }^{3}$ Hospital Universitario de Torrejon, Neumology, Madrid, Spain \\ ${ }^{4}$ Hospital Universitario de Torrejon, Nephrology, Madrid, Spain \\ ${ }^{5}$ Hospital Universitario de Torrejon, Internal Medicine, Madrid, Spain
}

Aims: To investigate how a resistant hypertension unit can improve blood pressure control.

Method: More than 350 patients were referred to our resistant hypertension unit from 2012-2015 to consider renal denervation therapy from our general practitioners, internal medicine, nephrology and neumology colleagues. After initial assessment, a standardised protocol was performed. 48 patients were finally considered for renal denervation.

Results: Patients were counselled for dietary and exercise recommendations, medication was adjusted (addition of antialdosteronic agents when indicated) with close follow-up visits, achieving good blood pressure control in $87 \%$ of the patients.

However, $13 \%$ of the patients (48) were finally screened for renal artery stenosis (CT scan) and sleep apnea (polysomnography) due to resistant hypertension: $16.7 \%$ had sleep apnea (new diagnosis) treated with continuous positive airway pressure; $14.5 \%$ had severe renal artery stenosis (unilateral / bilateral) treated with renal angioplasty; $8.3 \%$ were not considered for renal denervation due to severe renal failure or dialysis; $6.3 \%$ refused the technique (renal denervation); $16.7 \%$ were discarded due to other causes (psychiatric complex diseases, alcohol abuse, low adherence to medication, lost to follow-up) and $25 \%$ continued under follow-up due to gradual improvement with medical therapy adjustments. Lastly, $8.3 \%$ were treated with bilateral renal denervation including polar arteries, with no periprocedural complications and progressive reduction of blood pressure during follow-up. 2 patients (4.2\%) are scheduled for renal denervation.

Conclusion: Careful patient selection is crucial for renal denervation. A resistant hypertension unit can unmask secondary causes of resistant hypertension and improve blood pressure control in the majority of the patients.

No conflict of interest 


\title{
HYPERTENSION
}

\section{PREVALENCE OF SLEEP APNEA DISORDERS AMONG PATIENTS WITH RESISTANT HYPERTENSION REFERRED FOR RENAL DENERVATION}

\author{
E. Alegria-Barrero $^{1}$, S. Alonso-Viteri², R. Teijeiro-Mestre ${ }^{1}$, J. Ruiz-Garcia' ${ }^{1}$, M.A. Blázquez ${ }^{1}$, \\ M. Casares ${ }^{3}$, M. Vega ${ }^{1}$, L. Borrego ${ }^{1}$, M.Á. San Martin ${ }^{1}$ \\ ${ }^{1}$ Hospital Universitario de Torrejon, Cardiology, Madrid, Spain \\ ${ }^{2}$ Hospital Universitario de Torrejon, Neumology, Madrid, Spain \\ ${ }^{3}$ Hospital Universitario Rey Juan Carlos, Interventional Radiology, Madrid, Spain
}

Aims: To evaluate the incidence and prevalence of sleep apnea disorders among patients referred to a resistant hypertension unit for renal denervation treatment.

Method: More than 350 patients were referred to our resistant hypertension unit from 2012-2015 to consider renal denervation therapy from our general practitioners, internal medicine, nephrology and neumology colleagues. After initial assessment, a standardised protocol was performed. 48 patients were finally considered for renal denervation.

Results: Patients were counselled for dietary and exercise recommendations, medication was adjusted (addition of antialdosteronic agents when indicated) with close follow-up visits, achieving good blood pressure control in $87 \%$ of the patients. However, $13 \%$ of the patients (48) were finally screened for renal artery stenosis (CT scan) and sleep apnea (polysomnography) due to resistant hypertension

48 patients were finally enrolled for renal denervation consideration. We discovered that 19 patients had severe sleep apnea (39.6\%). Among these 19 patients, $11(58 \%)$ have been already diagnosed and were under continuous positive airway pressure (CPAP) treatment. 2 of these subgroup of 19 patients were non-adapted to the technique and were helped through specific CPAP whorkshops conducted by trained nurses, improving adherence and improving blood pressure control. 8 patients (42\%) were diagnosed during screening (new cases), giving a $16.67 \%$ incidence of sleep apnea among our study cohort.

After CPAP treatment, 2 out of 19 patients (10.5\%) had resistant hypertension and underwent renal denervation, with improvement of blood pressure control and no complications.

Conclusion: Sleep apnea is particularly prevalent among patients with resistant hypertension and deserve specific treatment before renal denervation consideration.

No conflict of interest 
310

\title{
HYPERTENSION
}

\section{POSTURAL VARIATION IN ARTERIAL STIFFNESS MEASUREMENT BETWEEN NON- HYPERTENSIVE AND HYPERTENSIVE SUBJECTS ON MEDICATIONS}

\author{
T.J. Quah, A. Sule, Q. Du \\ Tan Tock Seng Hospital, General Medicine, Singapore, Singapore
}

\begin{abstract}
Aims: Arterial stiffness which is closely related to cardiovascular events can be assessed by augmentation index (Al). Postural variations of $\mathrm{Al}$ were observed. We observed that the $\mathrm{Al}$ increased in supine position in most non-hypertensive volunteers. The current ongoing study compares measurement of $\mathrm{Al}$ in relation to posture in hypertensive and non-hypertensive subjects.
\end{abstract}

Method: To date, this ongoing study has recruited patients without hypertension $(n=21)$ and hypertensive subjects $(n=21)$. Als were analysed in both supine and sitting position. We compared this observation between non-hypertensive and hypertensive subjects; and subsequently amongst hypertensive patients to observe the effects of different classes of antihypertensives. Multiple Al measurements were done by SphygmoCor, which is the gold standard for non-invasive central pressures assessment. Wilcoxon signed-rank test and paired t test were used for statistical analysis with statistical significance set at the $p$ value of $<0.05$.

Results: In non-hypertensive subjects, aortic Al significantly increased from sitting to supine position $(\mathrm{P}<0.05)$, whereas significant postural changes of Al was not seen in hypertensive patients. On the contrary, Al significantly decreased with posture from sitting to supine in subgroup of patients on ACE inhibitors $(P=0.05)$.

Conclusion: Our findings suggest that $\mathrm{Al}$ increases in supine posture in subjects who are not on treatment. These findings may suggest that patients who are pre-hypertensives or hypertensives without treatment may have a greater increase in supine Al; thus predisposing them to early morning cardiovascular events but this effect is abolished or blunted when patients are on antihypertensives. Further studies need to be done to establish such correlations.

No conflict of interest 
311

\title{
HYPERTENSION
}

\section{UNTREATED HYPERURICEMIA IS ASSOCIATED WITH UNCONTROLLED HYPERTENSION AND PREVALENCE OF OTHER MAJOR CARDIOVASCULAR RISK FACTORS IN PRIMARY CARE SETTINGS}

\author{
A. Dukat ${ }^{1}, \underline{\text { P. Sabaka }}{ }^{1}$, J. Gajdosik', F. Simko ${ }^{3}$ \\ ${ }^{1}$ Comenius University in Bratislava- Faculty of Medicine in Bratislava, \\ 2nd Department of Internal Medicine, Bratislava, Slovakia \\ 2Private Office of General Practitioner, Private Office of General Practitioner, Nové Zámky, \\ Slovakia \\ ${ }^{3}$ Comenius University in Bratislava- Faculty of Medicine in Bratislava, \\ Department of Pathophysiology, Bratislava, Slovakia
}

\begin{abstract}
Aims: Many studies had indicated that hyperuricemia may increase the risk of development of cardiovascular diseases and its complications. Association of hyperuricemia with a presence of traditional cardiovascular risk factors has been also observed. Our goal was to assess a prevalence of untreated hyperuricemia and its association with other cardiovascular risk factors and hypertension control in patients with a history of arterial hypertension in large nationwide epidemiologic study.
\end{abstract}

Methods: Patients with the history of arterial hypertension from 330 offices of general practitioners in Slovakia were screened for untreated hyperuricemia. Serum creatinine, triacylglycerol, LDL, HDL, heart rate BMI, waist circumference and blood pressure were also measured.

Results: 19644 patients (46.06\% men and 53.94\% women) were recruited. The prevalence of untreated hyperuricemia was $17.3 \%$ in men and $20.0 \%$ in women. Its presence was associated with uncontrolled hypertension $(p<0.0001, O R=1.31)$ and hydrochlorothiazide treatment $(p<$ $0.0001, \mathrm{OR}=1.29$ ). Uric acid concentration correlated with $\mathrm{BMI}$, waist circumference, systolic and diastolic blood pressure, serum creatinine concentration, TAG, LDL and inversely correlated with HDL concentration.

Conclusions: Untreated hyperuricemia common finding among patents with arterial hypertension in primary care settings. It is associated with poorer hypertension control and correlates with other traditional cardiovascular risk factors. Hydrochlorothiazide treatment is associated with the presence of untreated hyperuricemia.

No conflict of interest 
312

\title{
HYPERTENSION
}

\section{WEIGHT REDUCTION AND WEIGHT GAIN \&NDASH; EFFECTS ON HYPERTENSION CONTROL DURING 1 YEAR FOLLOW UP}

\author{
P. Sabaka ${ }^{1}$, A. Dukát ${ }^{1}$, J. Gajdošík ${ }^{2}$, F. Šimko ${ }^{3}$, M. Čaprnda ${ }^{1}$, M. Bend'žala ${ }^{1}$, A. Komorníková ${ }^{1}$ \\ ${ }^{1}$ Comenius University- Faculty of Medicine in Bratislava, 2nd Department of Internal Medicine, \\ Bratislava, Slovakia \\ ${ }^{2}$ Private Office of General Practitioner, Private Office of General Practitioner, Nové Zámky, \\ Slovakia \\ ${ }^{3}$ Comenius University- Faculty of Medicie in Bratislava, 3rd Department of Internal Medicine, \\ Bratislava, Slovakia
}

\begin{abstract}
Aims: Obesity has been linked to increased risk of development of arterial hypertension and gaining weight is associated with increase of systolic and diastolic blood pressure. Aim of our prospective observational study was to assess an association of changes of body mass index (BMI) with hypertension control in patients with a history of arterial hypertension.
\end{abstract}

Method: We conducted prospective observational study. Patients with the history of arterial history were recruited from the offices of general practitioners. Patients were followed up for 12 months. During the follow up period, general practitioners were free to change the therapy. Body weight, height and blood pressure were obtained after recruitment and at the end of the follow up. Hypertension control was defined as systolic blood pressure below $140 \mathrm{mmHg}$ and diastolic blood pressure below $90 \mathrm{mmHg}$.

Results: We recruited 19644 patients (46.06\% men and 53.94\% women) with the history of arterial hypertension. Among obese patients with uncontrolled hypertension, decrease of $\mathrm{BMI}$ of more than $1 \mathrm{~kg} / \mathrm{m} 2$ was associated with higher probability of achieving hypertension control during follow up $(p<0.0001, R R=1.224, n=3375)$. Among patients with controlled hypertension at the baseline, increase of BMI more than $1 \mathrm{~kg} / \mathrm{m} 2$ was associated with higher risk of having uncontrolled hypertension after 12 months $(p<0.0001, R R=1.152, n=1469)$.

Conclusion: Weight reduction is important part of complex management of arterial hypertension in obese patients.

No conflict of interest 
313

\section{HYPERTENSION}

\section{EFFECT OF HEART RATE VARIABILITY BIOFEEDBACK ON BRAIN ELECTRICAL ACTIVITY IN STAGE I HYPERTENSIVE PATIENTS}

M. Sorokina, B. Koichubekov

Karaganda State Medical University, Medical biophysics and informatics, Karaganda, Kazakhstan

Aims: The assessment of the functional activity of the brain in hypertensive patients is a prerequisite for an adequate treatment, but it is often limited to blood pressure parameters monitoring and rarely used in practice. Nevertheless, the proper functioning of the central nervous system is precisely a reaction to any influence, including therapy and biofeedback intervention, and allows for quality evaluation of the selected treatment. We aimed to determine the efficiency of combining heart rate variability biofeedback (HRV-BF) with pharmacological therapy in order to keep the normal brain activity in stage I hypertensive patients.

Method: For this study fifty men, ages 40 to 60 , with stage 1 hypertension were selected by physicians. The participants were randomly put into two groups: receiving medication only (control group, $n=25$ ) and receiving medication with HRV-BF (study group, $n=25$ ). The study group participated in 20 sessions of HRV-BF. Electroencephalogram (EEG) was recorded for both groups: before treatment started and after HRV-BF intervention.

Results: The changes in EEG wave structure between control and study groups differed after 3 weeks. In the control group alpha activity significantly decreased $(p<0.05)$ in right frontal, parietal and occipital areas. Delta activity increased in left parietal and occipital areas, theta activity increased in the left occipital area. Brain electrical activity in the study group has not changed significantly.

Conclusion: Diffuse reduction in alpha activity, increase in slow-wave activity and other signs of EEG desynchronisation did not appear in stage I hypertensive patients treated with combination of HRV-BF and medication.

No conflict of interest 
314

\title{
HYPERTENSION
}

\section{EFFECTS OF HEART RATE VARIABILITY BIOFEEDBACK ON SYMPATHOVAGAL MODULATION IN HYPERTENSIVES}

\author{
M. Sorokina, B. Koichubekov \\ Karaganda State Medical University, Medical biophysics and informatics, Karaganda, Kazakhstan
}

\begin{abstract}
Aims: Stress can cause hypertension through repeated blood pressure elevation. It stimulates nervous system to produce large amounts of vasoconstricting hormones, which increase blood pressure. Early studies demonstrate that heart rate variability biofeedback (HRV-BF) sessions reduce the perceived stress. Our aim was to investigate whether HRV-BF results have equivalent effects on autonomic cardiovascular responses control during stressful conditions in hypertensives.
\end{abstract}

Method: For this study fifty men, ages 40 to 60 , with stage 1 hypertension, were observed while taking antihypertensive drugs. The parameters of HRV before and after $20 \mathrm{HRV}$-BF sessions were measured.

Results: After HRV-BF session total power (TP) of spectral density has changed due to parasympathetic system activation. TP has significantly changed from $(1436 \pm 99,55)$ to $(2843 \pm 334,3) \mathrm{ms} 2,(p<0,01)$, i.e. 1,98 times. As the result, spectral components grew significantly: HF (1,97 times), LF (1,92 times) and VLF (2,38 times), $(p<0,05)$. VLF-component has undergone the greatest change, which points to the involvement of higher cortical and subcortical centers of the regulation with normalisation of vegetative homeostasis, resulting from patients' state self-control.

Conclusion: HRV-BF sessions reduce stress index and normalise autonomic balance. It should be noted that although during HRV-BF sessions in this subgroup a significant decrease of heart rate was achieved $(p<0,01)$, the average heart rate after the sessions still exceeds the normal values.

No conflict of interest 


\title{
315 \\ DIAGNOSIS, DIAGNOSTIC METHODS \\ MANAGEMENT OF ISOLATED BRACHIOCEPHALIC TRUNK DISSECTION EXTENDED TO RIGHT INTERNAL CAROTID ARTERY
}

\author{
D. Voiculescu, D.C. Badiu
}

University of Medicine and Pharmacy "Carol Davila" Bucharest, MEDICINE, Bucharest, Romania

\begin{abstract}
Aims: A 46 years old man known with hypertension and G6PDH deficit, with no history of trauma complained of a sudden onset of anterior thoracic and right arm pain. Clinical exam showed intermittent right radial pulse absence and inter-arm BP difference without right arm motor deficit or any neurologic sign of stroke.
\end{abstract}

Method: Contrast enhanced computer tomography (CT), Carotid duplex ultrasound (CDU), transoesophageal echocardiography (TEE) and aortography

Results: CT showed dissection flap originating from the brachiocephalic trunk and continuing into the extracranial segment of the right common carotid artery with intermittent obstruction of the subclavian artery and no involvement of the aortic arch. CDU, TEE and aortography confirmed the absence of AA or right vertebral artery involvement, with only extracranial segment of internal CA involvement. Transthoracic echocardiography excluded dissection of the ascending aorta. Contrast Aortography was performed showing normal coronary arteries.

Conclusion: Isolated BT dissection is an extremely rare but apparently existing condition. Even if our patient was hypertensive and had a history of infection, we considered interesting the association between G6PDH deficiency and brachiocephalic trunk dissection, because of the intermittent anemia with secondary elevation of BP and inflammatory predisposing condition. Surgery has a limited role in this case, and is needed only when medical treatment with antiplatelet therapy does not control the ischemic events. The learning point of the case is the importance of an accurate detection of the anatomy of the vessels affected by dissection, because this makes a big therapeutic strategy difference. Aortography plays the most important role.

This paper was co-financed from the European Social Fund, through the Sectorial Operational Programme Human Resources Development 2007-2013, contract POSDRU/159/1.5/S/155463.

No conflict of interest 


\title{
316
}

\section{DIAGNOSIS, DIAGNOSTIC METHODS}

\section{H NMR RELAXOMETRIC STUDIES OF ERYTHROCITE MEMBRANE PERMEABILITY IN PATIENTS WITH CARDIOVASCULAR PATHOLOGIES WITH DIFFERENT PATHOGENIC MECHANISMS}

\author{
F. Revnic ${ }^{1}$, C.R. Revnic ${ }^{2}$, C. Ginghina ${ }^{2}$, A.S. Nica ${ }^{3}$, S. Voinea ${ }^{4}$, C. Pena ${ }^{1}$, B. Paltineanu ${ }^{5}$ \\ ${ }^{1}$ NIGG"Ana Aslan", Biology of Aging, Bucharest, Romania \\ 2UMF"Carol Davila", Cardiology, Bucharest, Romania \\ "UMF"Carol Davila", Rehabilitation, Bucharest, Romania \\ "UMF"Carol Davila", Oncology, Bucharest, Romania \\ ${ }^{5}$ UMF Tg.Mures, General Surgery, Bucharest, Romania
}

Aims: The aim of this study was to investigate by Nuclear Magnetic Resonance (NMR) erythrocyte membrane permeability in patients with cardiovascular pathologies with different pathogenic mecanisms: such as arterial hyperthension (AHT) atherosclerosis (AS) and chronic ischemic cardiomyopathy $(\mathrm{CIC})$.

Method: MR measurements of T2a - intraerythrocyte water protons relaxation time and Esed activation energy of intraerythrocyte proton relaxation processes were done on erythrocyte membranes from 773 patients with different cardiovascular pathologies from Cardiovascular Clinique versus controls with an AREMI pulse Spectrometer at a frequency of $25 \mathrm{MHz}$ using the standard sequence CARR-PURCELL-MEIBOOM-GILL with a 1-msec interval between pulses.

Results: In (AHT) women and men age 45-65, stage III-IV and in > 65 men, intraerythrocyte water structure characterized by (T2a) is deteriorated, the values being higher than normal, (AHT) young men being unaffected at this level. In $(\mathrm{CIC})$ there are changes in the internal water structure (T2a) of the erythrocytes more obvious in women but also present in men $<45$ and $>$ 65. In (AHT) Esed is increased in both sexes at middle age and only in stages III-IV for young patients .In (AS) T2a is increased in women despite of age and exhibits the highest T2a value in old men associated with lower values for Esed of water transport through the erythrocyte membrane.

Conclusion: NMR results are validated for $(\mathrm{AHT})$ evolution stage characterization both in men and women and for assessing the risk of possible complications which may occur. NMR permeability tests, might be helpful for the prevention of strokes and for finding the risk context of other cardiovascular diseases

No conflict of interest 
317

\title{
DIAGNOSIS, DIAGNOSTIC METHODS
}

\section{LEUKOCYTOSIS AND ANEMIA DETERMINE CARDIAC REHABILITATION OUTCOMES IN POST-STEMI PATIENTS}

\author{
R. Irzmanski, J. Kapusta, J. Kowalski, A. Obrebska, A. Mejer \\ Medical University, Clinic of Internal Medicine and Cardiac Rehabilitation, Łodz, Poland
}

Aims: The aim of this work was to apply basic laboratory blood tests for predicting the outcomes of cardiac rehabilitation in post- STEMI patients using a specially developed index

Method: The study involved 130 post-STEMI patients, including 97 males and 33 females aged $60.83 \pm 12.23$, admitted to the Clinic for cardiac rehabilitation. Upon admission, the patients underwent complete blood count and had fasting glucose and creatinine levels in blood serum determined (for GFR). All patients performed a cardiac stress test twice, before and after rehabilitation, to assess its effects.

Results: Leukocytosis and anemia were found to be the strongest determinants of the risk of cardiac rehabilitation failure $(O R=4.78$ and $O R=2.28 ; p=0.01$, respectively). They were used to construct a cardiac rehabilitation failure index, returning values from 0 to 2 . 0 - neither leukocytosis nor anemia present in a post-STEMI patient; 1 - either leukocytosis or anemia present in a post-STEMI patient; this results in a 2.76 -fold increase in the risk of cardiac rehabilitation failure; 2 - both leukocytosis and anemia present in a post-STEMI patient; this results in a 7.61-fold increase in the risk of cardiac rehabilitation failure.

Conclusion: A complete blood count prior to the beginning of a cardiac rehabilitation program is useful in predicting its final outcome. Leukocytosis and anemia are the strongest determinants of the final outcome of cardiac rehabilitation in post-STEMI patients. The cardiac rehabilitation failure index may be helpful in programing physical exercise to optimize rehabilitation outcomes while ensuring safety for post-STEMI patients.

No conflict of interest 
318

\title{
DIAGNOSIS, DIAGNOSTIC METHODS
}

\section{THE IMPACT OF HYDROTHERAPY ON CHANGE VASCULAR PERFUSION WITHIN THE LOWER LEGS AND THE QUALITY OF LIFE OF PATIENTS WITH PERIPHERAL CIRCULATORY DISORDERS}

\author{
R. Irzmanski, J. Kapusta, J. Kowalski, L. Pawlicki \\ Medical University, Clinic of Internal Medicine and Cardiac Rehabilitation, Łodz, Poland
}

\begin{abstract}
Aims: The aim of the study was to assess the impact of hydrotherapy on change vascular perfusion within the lower legs and the quality of life of patients with peripheral circulatory disorders.
\end{abstract}

Method: The study involved 105 patients with peripheral circulatory disorders (I,II according to the Fontaine classification) including 65 males and 40 females aged $62.74 \pm 11.34$, admitted to the Clinic. In group I and II underwent series of 10 whirlpool massage of the lower extremities, group III took part in the individually selected training program includes breathing exercises and free active exercises. Treatment results were assessed by chest impedance plethysmography and comparing the linear circumferences, ranges of motion and temperature of the lower limbs before and after the treatment.

Results: The temperature increase in lower extremities by an average of $0.5^{\circ} \mathrm{C}$, reducing edema thereby improving joint mobility. In the range of flow parameters PAmpl and PSlope seen an increase in value, while over a range of CT and PT their reduction compared to baseline. In group II significant changes in six minute test corridor compared with the control group (increase the distance of 159 meters $(p<0.05))$. SF-36 questionnaire showed improvement in the quality of life in all groups.

Conclusion: The results indicate that the whirlpool by the heat and mechanical impact on the improvement of lower limb blood flow, reduce swelling and venous stasis thereby having a positive impact on the functioning and quality of life of patients with peripheral circulatory disorders of the lower limbs.

No conflict of interest 
319

\title{
DIAGNOSIS, DIAGNOSTIC METHODS
}

\section{SAFETY AND COST EFFECTIVENESS OF OUTPATIENT TRANSRADIAL CORONARY ANGIOGRAPHY: A ONE-YEAR SINGLE-CENTER EXPERIENCE FROM THE PHILIPPINES}

\author{
J.K. Lerios, M. Sabas, R. Ramboyong, K.G. Floren \\ The Medical City, Section of Cardiology-Department of Medicine, Pasig, Philippines
}

Aims: Overnight admission prior to or after coronary angiography is still being done locally in most centers despite advances in technology which allow for same-day discharge worldwide. The aim of this study was to evaluate the safety of outpatient transradial coronary angiography and to review its cost-effectiveness compared with overnight admission in the local setting.

Method: This is a retrospective study from January to December 2014 on 68 consecutive patients who underwent elective outpatient transradial coronary angiography. Seven of them were subsequently admitted. We reviewed the charts of 62 corresponding inpatients to compare the occurrence of procedural complications between the two groups.

Results: There were older subjects in the outpatient group (t -2.751 , df $121, \mathrm{p} .007$ ) as well as greater prevalence of hypertension $(x 2=6.044$, p0.014), diabetes mellitus $(x 2=11.076, p$ 0.001 ), and three-vessel coronary artery disease ( $p$ 0.028). Procedure times (t $3.041, \mathrm{df} 121, \mathrm{p}$ .003 ) and time to removal of transradial closure device (t 8.155, df 121, $p<0.05$ ) were significantly longer among the inpatients. The crossover rate was only $0.77 \%$ in total, with no major adverse cardiovascular events in both groups. Four (4.41\%) in the outpatient group were admitted after ad-hoc angioplasty. The hospital-related cost of the procedure is a minimum of $7.83 \%$ less on an outpatient than inpatient basis.

\begin{tabular}{|c|c|c|c|}
\hline & Outpatient $(n=61\})$ & Inpatient $(n=62)$ & $P$ value \\
\hline Heart rate during vascular access, bpm & $71.98 \pm 14.27$ & $73.2 \pm 13.81$ & 0.638 \\
\hline SBP during vascular access, $\mathrm{mm} H \mathrm{~g}$ & $141.5 \pm 20.99$ & $136.2 \pm 20.55$ & 0.160 \\
\hline DBP during vascular access, $\mathrm{mmHg}$ & $83.69 \pm 11.37$ & $85.39 \pm 11.45$ & 0.411 \\
\hline MAP during vascular access, $\mathrm{mmHg}$ & $102.68 \pm 12.85$ & $102.31 \pm 13.4$ & 0.876 \\
\hline Vascular Access Time", mins & $3.95 \pm 4.91$ & $3.35 \pm 3.38$ & 0.434 \\
\hline Procedure durationtimes, mins & $14.49 \pm 6.86$ & $19.27 \pm 10.22$ & 0.003 \\
\hline Time to transradial device removal', mins & $199.65 \pm 4.4 .83$ & $323.61 \pm 110.52$ & $<0.05$ \\
\hline Crossover rate & 0 & $1(1.6196)$ & - \\
\hline \multicolumn{4}{|l|}{ Diagnosis after catheterization, $n$ [96] } \\
\hline Angiographically normal coronaries & $8(13.1196)$ & $13(20.9796)$ & 0.247 \\
\hline Mild CAD & $20(32.7996)$ & $33(53.2396)$ & 0.022 \\
\hline Single-vesselCAD & $10(16.3996)$ & $4(6.4596)$ & 0.083 \\
\hline Two-vessel CAD & $4(6.5696)$ & $2(3.2396)$ & 0.391 \\
\hline Three-ressel CAD & 15 (24.5996) & $6(9.6896)$ & 0.028 \\
\hline Myocardial Bridging & $5(8.2096)$ & $4(6.4596)$ & 0.710 \\
\hline Immediate postprocedural complications & $\mathrm{N}=18$ & $N=62$ & \\
\hline Major adverse cardiovascular events & 0 & 0 & - \\
\hline Access site hematoma & $6(33.3396)$ & $2(3.2396)$ & $<0.05$ \\
\hline Access site pain & $10[55.5696]$ & $17(27.4296)$ & 0.002 \\
\hline
\end{tabular}


Conclusion: Outpatient transradial coronary angiography is safe and cost-effective even among patients with multiple risk factors and stable multivessel coronary artery disease.

No conflict of interest 


\title{
CARDIAC SURGERY
}

TYPE A AORTIC DISSECTION AND POSTOPERATIVE MALPERFUSION: ANALYSIS OF INHOSPITAL AND LONG-TERM RESULTS IN A REFERENCE-CENTER FOR AORTIC EMERGENCY SURGERY

\author{
P. Nardi, C. Olevano, E. Bovio, C. Bertoldo, C. Bassano, A. Pellegrino, A. Scafuri, G. Ruvolo \\ Cardiac Surgery Unit, Tor Vergata University Policlinic, Rome, Italy
}

Objectives: The aim of this study is to evaluate the effect of postoperative malperfusion (PM) on in-hospital mortality and at long-term follow-up in patients undergone surgery for acute type A aortic dissection.

Methods: We examined data of 214 patients (mean age $62.5 \pm 12.6$ years, 156 males) operated on from January 2005 to March 2014 for aortic emergency surgery. At presentation, various types of malperfusion (cerebral, renal, mesenteric) were present in 119 patients (55.6\%). Arterial access for cardiopulmonary bypass was via femoral artery $(n=99)$, via axillary artery $(n=99)$, into the ascending aorta $(n=22)$. Aortic repair was performed using "open technique" in 124 patients $(58 \%)$.

Results: Fifty-five patients (25.7\%) developed PM; overall in-hospital mortality was $29 \%$ (62/214): $47.3 \%$ in PM patients vs. 22.6\% in non-PM patients (P75 years (OR:1.1,Cl:1.03-1.13, $\mathrm{P}=0.0004)$ and renal $\mathrm{PM}(\mathrm{OR}: 53.5, \mathrm{Cl}: 3.97-721.3, \mathrm{P}=0.0027)$. Five-year survival was $79 \pm 7 \%$ in PM patients vs. $94 \pm 3 \%$ in non-PM patients (Log-rank test, $P=0.0002$ ). Independent predictors for survival were age $>75$ years (OR:375, Cl:0.9-14.0, $\mathrm{P}=0.05$ ) and renal $\mathrm{PM}(\mathrm{OR}: 28.6, \mathrm{Cl}: 1.8-462.0$, $\mathrm{P}=0.01$ ); all types of $\mathrm{PM}$ and distal location of intimal tear were found as risk factors at the univariate analysis.

Conclusions: Surgery of acute aortic dissection is effective in reducing by about $50 \%$ preoperative malperfusion. Postoperative renal malperfusion is associated with high in-hospital mortality and reduced long-term survival. Surgical techniques, site of cannulation, more complex interventions requiring "open technique" did not show to be predictors of increased risk. .

No conflict of interest 


\title{
CARDIAC SURGERY
}

\section{AORTIC SURGERY WITH CORONARY REVASCULARIZATION FOR ACUTE TYPE A DISSECTION}

\author{
Y. Takahara, K. Mogi, M. Sakurai \\ Funabashi Municipal Medical Center, \\ Division of Cardiovascular Surgery in Heart and Vascular Institute, Funabashi, Japan
}

Aims: We evaluated early and long-term outcomes following aortic surgery for acute type $A$ dissection with or without coronary revascularization comparatively.

Method: From 2005 to 2014, 113 consecutive patients underwent aortic replacement for acute type $A$ dissection. The 22 patients underwent simultaneous coronary revascularization (Group A). The other 91 patients consisted of the control group (Group B). In the Group A, 9 patients with occlusion of the coronary orifice due to aortic dissection underwent trimming of dissection or reconstruction of the coronary orifice, 10 patients with annuloaortic ectasia underwent reconstruction of the coronary orifice, and 3 patients with coronary artery disease underwent CABG.

Results: There were one hospital death (4.5\%) in the Group A and 4 hospital death $(4.4 \%)$ in the Group B. The rates of major cerebral complications were $9.1 \%$ vs. $5.5 \%$. Survival rates at 5 years after the operation were $89.1+/-7.4 \%$ vs. $88.6+/-4.5 \%$. Freedoms from cardiovascular events at 5 years after the operation were $63.3+/-12.4 \%$ vs. $88.9+/-3.6 \%$ ( $p=0.0465$ Log Rank). In the subgroup analysis of the Group A, the freedom rate from cardiovascular event in the preoperative ST-elevation group was lower than in the non ST-elevation group, significantly $(p=0.0468$ Breslow).

Conclusion: There were no significant difference of early outcomes and long-term survival rates between the two Groups. However, the freedom rate from cardiovascular event in the Group A was significantly lower than in the Group B.

No conflict of interest 


\title{
CARDIAC SURGERY
}

\section{STAGED OPERATION FOR CORONARY ARTERY DISEASE AND ABDOMINAL AORTIC ANEURYSM COMPLICATED WITH CONGENITAL DUAL LEFT ANTERIOR DESCENDING CORONARY ARTERY}

\author{
S. Yamashiro, R. Arakaki, Y. Kise, H. Inafuku, Y. Kuniyoshi \\ Graduate School of Medicine - University of the Rykyus, \\ Department of Thoracic and Cardiovascular Surgery, Nishihara-cho- Nakagami-gun, Japan
}

Aims: A dual left anterior descending artery is an extremely rare congenital coronary anomaly that has no clinical significance unless superimposed with atherosclerotic coronary artery disease.

We describe a 71-year-old Caucasian male with a dual left anterior descending artery who presented with angina pectoris and a gradually expanding abdominal aortic aneurysm.

Method: Coronary angiography revealed a Type 3 dual left anterior descending coronary artery and three-vessel disease with left main trunk stenosis. The optimal treatment of patients with critical coronary artery disease and a large or symptomatic abdominal aortic aneurysm remains controversial. We scheduled a staged operation to treat the rapidly expanding abdominal aortic aneurysm and coronary disease in our patient because the one-stage on-pump approach is associated with increased risk of bleeding and other overlapping complications that might be attributable to either procedure.

Results: We initially treated the abdominal aortic aneurysm by endovascular aortic aneurysm repair because it had expanded by about $5 \mathrm{~mm}$ during short period. Two weeks later, the patient underwent a coronary artery bypass graft.

Conclusion: We considered that the management strategy selected for this patient was apparently optimal. Compared with conventional surgical approaches, endovascular aortic aneurysm repair followed by coronary artery bypass grafting was less invasive.

This rare coronary artery anomaly is clinically important from the viewpoint of myocardial revascularization.

No conflict of interest 


\title{
CARDIAC SURGERY
}

\section{CORONARY ARTERY STENTING AND CARDIAC SURGERY A COMPARATIVE OUTCOME STUDY}

\author{
J. Garcia-Carro, J.M. Martinez Cereijo, J. Siera Quiroga, B. Adrio Nazar, L. Reija López, \\ Á.L. Fernández González, D. Durán Muñoz \\ Universitary Hospital Santiago de Compostela, Cardiac surgery, Santiago de Compostela, Spain
}

\begin{abstract}
Aims: Increasingly patients are being referred for cardiac surgery after previous percutaneous coronary stenting (PCS). Recent studies suggested that patients with PCS had a higher incidence of death after CABG. We did a comparative study to determine the impact of this on patients outcome after cardiac surgery.

Method: 120 patients with PCS undergoing cardiac surgery (group 1), were evaluated for in hospital mortality and morbidity and compare with 120 patients undergoing cardiac surgery without PCS (group 2).
\end{abstract}

Results: Patients groups did no differ according to most of their preoperative demographics risk factors. Time between stenting and surgery was shorter than 3 months in $46 \%$ of patients, $54 \%$ were bare-metal stents and $46 \%$ were drug-eluting stents .Group 1 patients had a higher incidence of early mortality $(P=0,002)$, postoperative myocardial infarction $(P=0,043)$, low cardiac output $(P=0,0001)$, major postoperative bleeding $(P=0,09)$, smaller luminal diameter distal to stent $(P=0,037)$. Patients with drug-eluting stents had a higher mortality $(p=N S)$ and a short time $(<3$ months) between stenting and surgery was a risk factor of mortality $(P=0,007)$.

Conclusion: PCS before cardiac surgery increases the risk for in hospital mortality and postoperative major adverse cardiac events. Short time between stenting and surgery in patients with drug-eluting stent had a worse prognosis.

No conflict of interest 


\section{CARDIAC SURGERY}

\section{RAPID PROGRESSION OF LMT STENOSIS AFTER AORTIC ROOT REPLACEMENT}

\section{R. Arakaki, Y. Kuniyoshi, S. Yamashiro}

University of the Ryukyus, Thoracic and Cardiovascular Surgery, Nishihara-cho- Nakagami-gun, Japan

Aims: The left main trunk (LMT) stenosis after aortic valve replacement or aortic root operations is a rare but potentially life-threatening complication. We describe a case of rapid progression of stenosis at distal site of LMT after Bentall operation.

Method: A 45-year-old man, who had undergone aortic valve replacement (AVR) with mechanical valve 15 years ago, admited due to ascending aortic aneurysm. Preoperative coronary angiography (CAG) revealed mild narrowing of distal LMT site (just proximal of left anterior descending). We performed Bentall operation. Both coronary arteries were reconstructed using Carrel's button technique. Myocardial protection was achieved by intermittent antegrade administration of cold blood cardioplegia through selective ostial cannulation. Postoperative course was uneventful and postoperative coronary CT showed no progression of stenosis at discharge.

Eight weeks after second operation, the patient suddenly complained severe chest pain. CAG showed severe stenotic lesion at the distal site of LMT.

Results: We performed the percutaneous coronary intervention $(\mathrm{PCI})$ in order to avoid the risks connected to early repeat sternotomy. IVUS revealed fibrous plaque. The $\mathrm{PCI}$ using drug-eluting stent (Nobori 3.5×18mm) was successfully performed.

Conclusion: Several causes of coronary artery stenosis after AVR or aortic root operations have been proposed. In our case, the presence of small plaque in the coronary artery might have caused progression of the stenosis. Although standard treatment of LMT stenosis might be coronary artery bypass grafting, we performed PCI treatment which have a concern of re-stenosis or occlusion. Strict follow-up is required to predict progression of coronary stenosis.

No conflict of interest 


\section{CARDIAC SURGERY}

THE IMPACT OF THE ANNUAL AUGUST TRAINEE CHANGEOVER ON CARDIAC SURGICAL OUTCOMES IN A SINGLE UK INSTITUTION

\section{E. Caruana, S. Nashef}

Papworth Hospital NHS Foundation Trust, Cardiothoracic Surgery, Cambridge, United Kingdom

Aims: It has been previously demonstrated that the month of August is associated with adverse patient outcomes, commonly attributed to trainee changeover and novice doctors. We sought to re-evaluate this phenomenon at a UK-based specialist cardiac surgical centre with a focus on long-term outcome.

Method: Prospectively-collected data was obtained for all patients who underwent cardiac surgery at our institution between January and December 2013. Student $t$ test and Pearson's Chisquared test were used for statistical analyses.

Results: 165 patients (8.5\% of the annual cardiac surgery workload) had operations in August. There was no difference in logistic EuroSCORE between August and the other months collectively (9.1 \pm 11.4 vs $9.8 \pm 12.4, p=0.496)$. Pre- operative $(2.1 \pm 3.7$ vs $2.5 \pm 4.6, p=0.353)$ and post-operative (10.2 \pm 6.0 vs $10.4 \pm 8.0)$ lengths of hospital stay were similar in both groups. Hospital mortality was higher in August (4.8 vs $2.0 \%, p=0.019)$. The difference at 90 -days $(6.1$ vs $4.1 \%, p=0.236)$ and at one-year ( 9.1 vs $6.5 \%, p=0.199)$ was not statistically significant.

Conclusion: In-hospital mortality is higher for operations in the month of August.

No conflict of interest 


\title{
CHRONIC HEART FAILURE AND PROGRESS OF COMBINED TREATMENT OF TRADITIONAL CHINESE MEDICINE AND WESTERN MEDICINE WITHIN LAST TEN YEARS
}

\author{
D. Yan, L. Wang, C. Yu, Y. Bian \\ Tianjin University of Traditional Chinese Medicine, Tianjin, China \\ Objective: To investigate traditional Chinese medicine (TCM) discriminates of chronic heart \\ failure and the progress of combined treatment of traditional Chinese medicine and western \\ medicine within last ten years.
}

Method: Referred to the recent literatures, summarizing TCM discriminate of chronic heart failure and the advantage of combining traditional Chinese and western medicine in the treatment of chronic heart failure

Result: Although there are many TCM discriminate of chronic heart failure, it could be divided into the following types: qi deficiency, qi and Yin dificiency, Yang deficiency based on asthenia in origin and asthenia in superficiality, in combined with pathological factors such as phlegm turbidity, retained fluid and congestion. Warming yang for diuresis, promoting blood circulation to remove blood stasis, boosting Qi and nourishing Yin are commonly used for the treatment of chronic heart failure in TCM. For combined traditional Chinese medicine and western medicine, the treatment of chronic heart failure is based on anti-hypertension, strong heart, dieresis, expansion coronary artery in combination with TCM discriminate treatment.

Conclusion: Through data integration we found that TCM discriminate have dramatic advantage in the treatment of chronic heart failure. On the basis of conventional western medicine treatment in combining with traditional Chinese medicine, can effectively improve heart function and relieve the clinical symptoms.

Key words: Heart failure; TCM discriminate; Chinese and western medicine

Acknowledgments: This project was supported by the National Basic Research Program of China (973 Program, 2014CB542902, http://program.most.gov.cn/). The funders had no role in study design and data collection and analysis, decision to publish, or preparation of the paper.

Document not received 


\title{
LV FUNCTION, HEART FAILURE
}

\section{CORONARY GIANT FISTULA AS A CAUSE OF VENTRICULAR DYSFUNCTION. A CASE REPORT}

\author{
V. Bonilla ${ }^{1}$, G. Eva ${ }^{2}$, E. Pablo ${ }^{3}$, G.L. Alberto \\ ${ }^{1}$ Hospital Principe de Asturias, Cardiolgy Department, Alcalá de Henares, Spain \\ ${ }^{2}$ Hospital Principe de Asturias, Cardiology Department, Alcalá de Henares, Spain \\ ${ }^{3}$ Hospital Principe de Asturias, Endocrinology Department, Alcalá de Henares, Spain \\ ${ }^{4}$ Hospital Principe de Asturias, Cardiology Department, Alcala de Henares, Spain
}

Aims: Coronary artery fistula is one of the most common congenital anomalies of the coronary arteries. More frequently are small and asymptomatic, however the large ones can cause complications.

Method: We report a patient admitted with left ventricular dysfunction secondary to giant coronary fistula

Results: Male 77 years. Hypertension and diabetes in drug treatment. Chronic renal failure. He was operated of abdominal aortic aneurysm. COPD with LTOT. Admitted in the emergency department for congestive heart failure, after cold symptoms. The ECG showed sinus tachycardia at $100 \mathrm{bpm}$ with LBBB. We decided to hospitalize for study and treatment. After negative balances, beginning of treatment with beta blockers, ACEI and antialdosterone, clinical improvement occurs. Echocardiography showed severe left ventricular dysfunction with marked intraventricular asynchrony, severe functional mitral regurgitation and severe pulmonary hypertension. Coronary angiography was requested showing ectatic DA that anastomoses with fistulous tract in its apical portion, which is directed by anterior right ventricule before emptying into the right pulmonary artery. The CX hadn't injuries and the CD was partly filled by collateral heterocoronaries and seemed underdeveloped giving the impression that ended partially flowing into the fistula described. Aortogram was performed and no origin of CD was displayed in the aortic root. Attending to his condition and high comorbidity we decided conservative management without therapy with CRT-ICD 


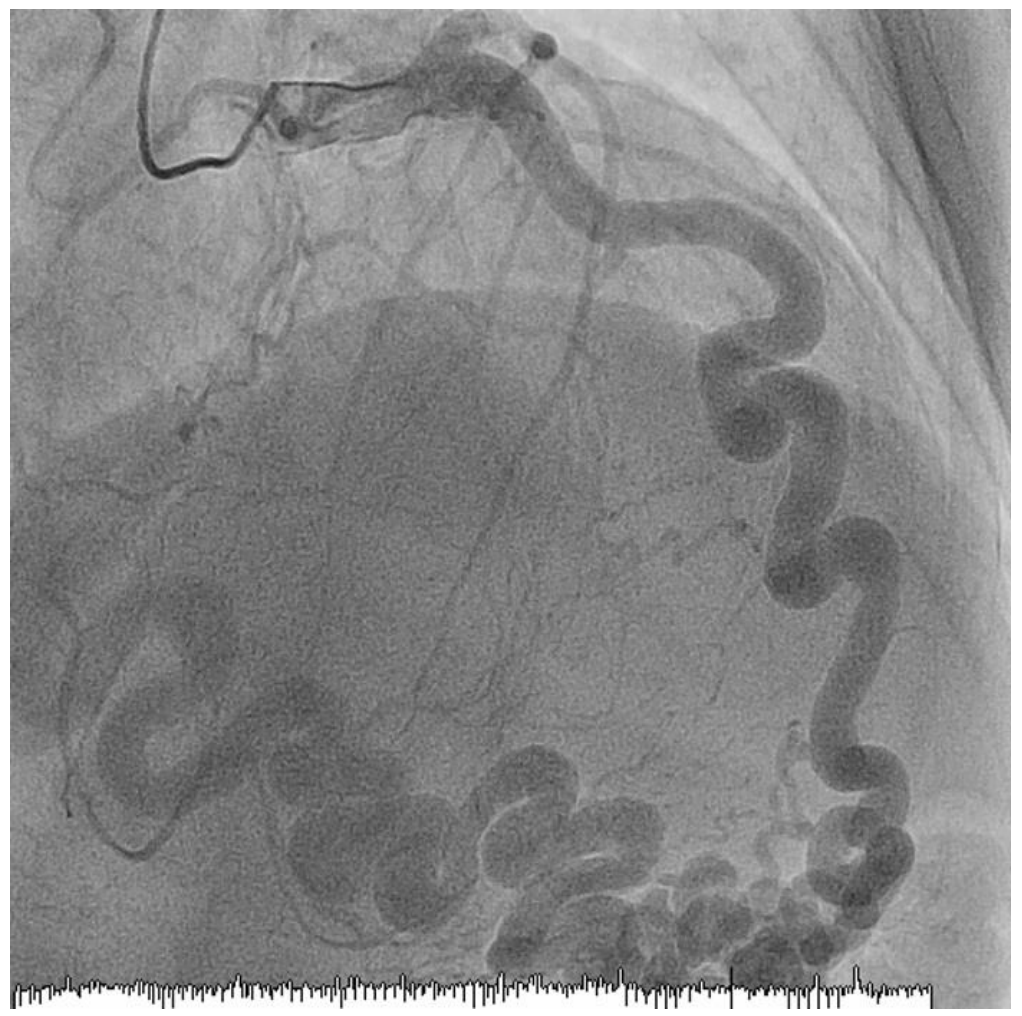

Conclusion: Nowadays, the percutaneous closure or surgery in multiple or large fistulas are considered first line treatment to avoid long-term complication as in this case

No conflict of interest 
330

LV FUNCTION, HEART FAILURE

\title{
MOLECULAR AND BIOPHYSICAL ASPECTS OF CARDIAC CONTRACTILITY IN PATIENTS WITH HEART FAILURE
}

\author{
F. Revnic ${ }^{1}$, C.R. Revnic ${ }^{2}$, C. Ginghina ${ }^{2}$, A.S. Nica ${ }^{3}$, S. Voinea ${ }^{4}$, C. Pena ${ }^{1}$, B. Paltineanu ${ }^{5}$ \\ ${ }^{1}$ NIGG"Ana Aslan", Biology of Aging, Bucharest, Romania \\ ¿UMF"Carol Davila", Cardiology, Bucharest, Romania \\ "UMF"Carol Davila", Rehabilitation, Bucharest, Romania \\ "UMF"Carol Davila", Oncology, Bucharest, Romania \\ ${ }^{5}$ UMF Tg. Mures, General Surgery, Targu Mures, Romania
}

\begin{abstract}
Aims: Ischemia induces changes in distribution and polarisation of tissue water and this may influence (NMR) proton transverse relaxation times. This study was concerned with molecular and biophysical aspects of cardiac contractility in patients with mitral valve insufficiency,(MVI)in order to improve our understanding of this condition and to learn how to deal with it.
\end{abstract}

Method: The investigation of ionic behaviour as well as the charge density(using Na22 uptake)radioisotope method upon contractile proteins from glycerinated left ventricle papillary muscle biopsies of patients with (H.F.) were performed in rigor and relaxation media by measuring proton transverse relaxing times $T_{2} S$ and $T_{2} /$ with an Aremi 78 Spectrometer at $25 \mathrm{MHz}$ frequency using a Carr-Purcell-Meiboom-Gill pulse sequence with 32 spin echoes ranging from 6-256 milliseconds after the 90 degree pulse.

Results: The increase in T2s and T2l in (MVI) patients does not derive from an increase in water concentration within muscle, and this can be accounted for a modification in quantity and quality of dissociable protein groups of contractile proteins.Supplimentary dissociation of $\mathrm{COO}$ - and $\mathrm{NH}+2$ of contractile proteins with modified features, determine an increase in superficial charges at the surface of myosin filaments.A decrease in fixed negative charge density in contraction at the surface of myosin filaments in (MVI) sarcomeres is followed by an increase in superficial charges due to lability of $\mathrm{COO}$ - and $\mathrm{NH}+$ protein groups.

Conclusion: The decrease in charge density upon contractile filaments with modified features from diseased myocardium means that a certain proportion of crossbridges are no longer functionally efficient for performing contraction .

No conflict of interest 
331

LV FUNCTION, HEART FAILURE

NTU-B PRESERVED CARDIAC BIOENERGETIC IN ISOPROTERENOL-INDUCED HEART FAILURE

S.Y. Lee

Mackay Memorial Hospital, Pulmonary and Critical Care Medicine, Taipei, Taiwan

Aim: Heart failure is defined as cardiac dysfunction because of cardiac structural or functional disorders. Cardiac bioenergetics and catecholamine play major roles in heart failure progression. The relationships between these factors are not fully understood.

Method: NTU-B, an anti-oxidant, was applied to mice under chronic isoproterenol (ISO) stimulation to realize its effect on heart failure. Cardiac function was detected by echocardiography. HL-1 cell was exposed to isoproterenol to induce stress.

Results: Administration of ISO in HL-1 cell decreased oxygen consumption rate and increased glycolysis rate, which decreased intracellular $\mathrm{PH}$ value and ATP production. Cardiac dysfunction was induced after 2-week of ISO administration in mice. The deterioration of cardiac function is associated with the loss of cardiac ATP content. NTU-B preserved oxidative phosphorylation, cellular bioenergetics in ISO treated $\mathrm{HL}-1$ cell, and subsequently improved cardiac function in ISO treated mice.

Conclusion: CAEA prevents catecholamine-induced cardiac damage and is therefore a possible new therapeutic approach for preventing heart failure progression.

No conflict of interest 
332

LV FUNCTION, HEART FAILURE

\title{
DETERMINANTS OF DOSE OF BETA-BLOCKERS IN PATIENTS WITH CHRONIC HEART FAILURE
}

\author{
R. Mitsutake, H. Urata, K. Okamura \\ Fukuoka University Chikushi Hospital, Cardiovascular Disease, Chikushino, Japan
}

Aims: Beta-blockers (BB) have been proved to reduce morbidity and improve survival in patients with chronic heart failure (CHF), and several studies have reported that these benefits are dosedependent. But Underuse of $\mathrm{BB}$ for $\mathrm{CHF}$ is often seen in real world conditions. We examined the determinant of and the dose of BB for CHF patients in our hospital.

Method: In this retrospective study, consecutive 118 hospitalized patients (male 54\%, mean 75 years) with CHF were enrolled from 2013 to 2014, and examined for the dose of BB at hospital discharge.

Results: Of the total 118 patients, 97 (82\%) were treated with RAS inhibitors, 87 (74\%) with loop diuretics, $78(66 \%)$ with BB, $54(46 \%)$ with aldosterone receptor blocker. BB included carvedilol $(74 \%)$ and bisoprolol (25\%). There were 40 patients (34\%) treated without BB. Mean dose of carvedilol and bisoprolol was $4.9 \mathrm{mg} /$ day and $2.2 \mathrm{mg} /$ day, respectively. During hospitalization (mean 17 days), body weight, blood pressure, and heart rate were significantly reduced at hospital discharge. When the dose of BB was divided into two groups (higher group, carvedilol $>5.0 \mathrm{mg} /$ day or bisoprolol $>2.5 \mathrm{mg} /$ day; lower group, carvedilol $<5.0 \mathrm{mg} /$ day or bisoprolol $<2.5 \mathrm{mg} /$ day), logistic regression analysis showed that age was negatively and use of aldosterone blocker was positively correlated with BB groups ( $p=0.0115$ and $p=0.0194$, respectively).

Conclusion: Treatment with $\mathrm{BB}$ for $\mathrm{CHF}$ patients was inadequate in our hospital, and underuse of BB was evident in older patients. However, use of BB for CHF should take into account the prognosis especially in older patients.

No conflict of interest 
333

LV FUNCTION, HEART FAILURE

\section{ANXIETY AND DEPRESSION IN HEART FAILURE PATIENTS: A PROSPECTIVE, CROSS- SECTIONAL STUDY}

N. Muljadi', M.F.L. Ramirez', F.R.G. De Leon², E. Raya-Ampil2

${ }^{1}$ University of Santo Tomas Hospital, Medicine, Manila, Philippines

${ }^{2}$ University of Santo Tomas Hospital, Neurology and Psychiatry, Manila, Philippines

Aims: This study determined the prevalence of anxiety and depression and its correlation with clinical profile in heart failure patients using the Hospital Anxiety and Depression Scale Filipino Version (HADS-P).

Method: A cross-sectional study was conducted. HADS-P questionnaire was administered to heart failure patients admitted at the University of Santo Tomas Hospital from December 2013 to November 2014. The recommended cut-off score for HADS-P is 11. Chi-square and Independent T-tests were used in this study.

Results: 144 patients were enrolled in this study. There were 71 male and 73 female with mean interval of age $62.95+13.28$. The prevalence of depression and anxiety among heart failure patients is $13.2 \%$ and $25.7 \%$, respectively. History of coronary angiography, coronary angioplasty, ejection fraction $<40 \%$, and multiple co-morbid diseases were significant factors for depression. The most significant factor for anxiety was the length of hospital stay.

Conclusion: This study showed that depression and anxiety were common among heart failure patients. A formal psychiatric evaluation may be done and should be part of the comprehensive management among heart failure patients with depression or anxiety.

No conflict of interest 
334

LV FUNCTION, HEART FAILURE

\title{
ALLOGENIC SPERMINE TREATED-ADIPOSE TISSUE DERIVED MULTI-LINEAGE PROGENITOR CELLS IMPROVE LEFT VENTRICULAR DYSFUNCTION IN A SWINE CHRONIC MYOCARDIAL INFARCTION MODEL
}

\author{
H. Okura ${ }^{1}$, N. Hasebe ${ }^{1}$, A. Matsuyama ${ }^{1}$ \\ ${ }^{1}$ National Institutes of Biomedical Innovation Health and Nutrition, \\ Platform for Therapeutics for Rare Disease, Amagasaki, Japan
}

Aims: We have reported the spermine-treated adipose tissue-derived multi-lineage progenitor cells (ADMPCs) could improve left ventricular dysfunction in a swine chronic myocardial infarction model. In this study, we examined whether not only the autologous but the allogenic sperminetreated ADMPCs could improve left ventricular dysfunction in a chronic myocardial infarction.

Method: From three swine, the subcutaneous adipose tissues were acquired. Then the tissues were digested with collagenase. Twenty-four hours later after seeding the cells were treated with EDTA-solution, and the detached cells were collected as ADMPCs and expanded. To build up chronic myocardial infarction, six swine including the donors received first ballooning and reperfusion to first diagonal branch and second one to left ascending coronary artery (\#6) 1 weeklater. Four week-later second one, these swine received transplantation of allogenic $(n=3$, mixture of the cells derived from three donors) or autologous $(n=3)$ spermine treated-ADMPCs, or lactic Ringer's solution via intracoronary (\#6), and echocardiogram was examined at 0, 4, 8 and 12 weeks after transplantation.

Results: Twelve-week-follow-up showed rescue of function in the transplanted in allogenic cell transplanted swine. There is no significant difference between the autologous and allogenic transplanted animals without immune suppressants in the recovery of ejection fraction. No adverse events, including immune-rejecting symptoms, had been observed in all animals, nor no inflammatory cell infiltration were observed in these two groups histologically.

Conclusion: The transplantation of allogenic spermine treated-ADMPCs without immunosuppressive regimens is a cost-effective therapeutic strategy for future cardiac tissue regeneration.

No conflict of interest 
335

LV FUNCTION, HEART FAILURE

THE RELATION OF BETWEEN PULMONARY HYPERTENSION AND DIASTOLIC FUNCTION IN PATIENTS WITH LEFT VENTRICULAR SYSTOLIC DYSFUNCTION

S.H. Seol, S. Guang-Won, S. Pul-Sang, K. Dong-Kie, K. Ki-Hun, K. Doo-Il

Inje University Haeundaepaik Hospital, Division of Cardiology, Busan, Korea

Aims: Pulmonary hypertension (PH) secondary to left ventricular systolic dysfunction (LVSD) is often a poor prognosis marker. Diastolic filling pressure abnormalities have been described in patients with severe $\mathrm{PH}$. The aim of our study was to assess between diastolic function and $\mathrm{PH}$ associated LVSD.

Methods: 355 patients with LV Ejection fraction (EF) $\leq 40 \%$ were divided into two groups according to the $\mathrm{PH}$. $\mathrm{PH}$ estimated with the right ventricular systolic pressure. $\mathrm{PH}$ was defined systolic pulmonary artery pressure (PAP) $>35 \mathrm{mmHg}$ at rest. Diastolic parameters (transmitral flow velocity (E)/mitral annular velocity (e'), mitral valve deceleration time (DT) and left atrium diameter (LAD) were evaluated.

Results: 180 patients had $\mathrm{PH}$, whereas 175 patients had normal. Patients with $\mathrm{PH}$ were with higher E/e` ratio, lower DT, LVEF and increased LAD. In multiple regression analysis, PAP was significantly correlated with E/e` ratio, DT and LAD. In multivariate analysis, E/e` ratio> 15(odds ratio: 5.5, $\mathrm{P}<0.001$ ), LAD (>4.2 cm in male, $>3.8 \mathrm{~cm}$ in female) (odds ratio: $2.8, \mathrm{P}<0.001$ ), DT $<150 \mathrm{~ms}$ (odds ratio: $2.8, \mathrm{P}=0.002$ ), female (odds ratio: $2.8, \mathrm{P}=0.005$ ) were the independent risk factors for $\mathrm{PH}$ in patients with LVSD.

Conclusions: The diastolic echocardiographic parameters may be important predictors for $\mathrm{PH}$ in patients with LVSD.

No conflict of interest 
336

\title{
LV FUNCTION, HEART FAILURE \\ INCREASED RISK OF HOSPITALIZATION FOR HEART FAILURE WITH NEWLY PRESCRIBED DPP4 INHIBITORS AND PIOGLITAZONE
}

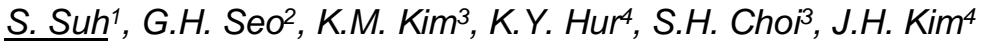 \\ ${ }^{1}$ Dong-A University College of Medicine, Division of Endocrinology and Metabolism, Busan, \\ Korea \\ ${ }^{2}$ Health Insurance Review and Assessment Service, \\ Health Insurance Review and Assessment Service, Seoul, Korea \\ ${ }^{3}$ Seoul National University College of Medicine, Division of Endocrinology and Metabolism, Seoul, \\ Korea \\ ${ }^{4}$ Sungkyunkwan University School of Medicine, Division of Endocrinology and Metabolism, Seoul, \\ Korea
}

Aims: We assessed the association of dipeptidyl peptidase 4 inhibitors (DPP4i) with hospitalization for heart failure (HF) using the Korean Health Insurance claims database.

Method: We collected data on newly prescribed sitagliptin, vildagliptin, and pioglitazone between January 1, 2009 and December 31, 2012 (mean follow-up of 336.8 days) to 935,519 patients with diabetes (518,614 males and 416,905 females) aged 40 to 79 years (mean age of 59.4 years).

Results: During the study, 998 patients were hospitalized for primary HF (115.7 per 100,000 patient-years). The incidence rate of hospitalization for HF was 117.7 per 100,000 per patientyears among patients on pioglitazone, 105.7 for sitagliptin, and 135.8 for vildagliptin. The hospitalization rate for HF was greatest in the first 30 days after starting the medication, which corresponded to a significantly higher incidence at days 0 to 30 compared with days 31 to 360 for all three drugs. The hazard ratios were 1.85 (pioglitazone), 2.00 (sitagliptin), and 1.79 (vildagliptin). The incidence of hospitalization for HF did not differ between the drugs for any time period.

Conclusion: This study showed an increase in hospitalization for HF in the initial 30 days of the DPP4i and pioglitazone compared with the subsequent follow-up period. However, the differences between the drugs were not significant.

No conflict of interest 
337

SURGICAL ASPECTS

\title{
SURGICAL TREATMENT IN SYNTOMATHIC ANOMALOUS ORIGIN OF RIGHT CORONARY ARTERY
}

\author{
A. Granda Bauza, A. Narvaez Muñoz, L. Reija Lopez, J.M. Martinez Cereijo, B. Adrio Nazar, \\ J. Garcia Carro, A. Fernández González, J. Sierra Quiroga, D. Duran Muñoz \\ Universitary Hospital Santiago de Compostela, Cirugía Cardíaca, Santiago de Compostela, Spain
}

\begin{abstract}
Aims: Anomalous origin of coronary arteries (AOCA) is known to cause intermittent ischemia, myocardial infarction and, sudden death. Published data suggests the range of $0.1 \%-0.3 \%$. Patient with AOCA are typically asymptomatic and symptomatic patients complain of syncope with exercise, chest pain, palpitations, even sudden death. The diagnosis is often made as an incidental finding.
\end{abstract}

Case: 42-year-old man, smoker, without others family or personal interesting antecedents. He was monitoring of chest pain in exercise. The coronariography showed that left coronary artery did not present significant angiographic lesions and right coronary artery (RCA) was dominant and with anomalous origin from left sinus of Valsalva and Computed tomography coronary angiogram revealed origin of right coronary artery from left coronary sinus. The artery turned immediately rightwards in a very acute angle and traversed in between pulmonary trunk and aorta. Was indicated CABG off- pump in the proximal right coronary artery was performed using the right internal mammary artery (RIMA). The flow was checked using transit-time ultrasound with proximal occlusion of RCA and without it. The improvement of the flow in the diastole also of the pulsatility index $(\mathrm{PI})$ after the proximal RCA ligature was demonstrated and the proximal RCA was ligated

Results: The postoperative course was favorable and he was discharged from hospital free of symptoms at the third day.

Conclusion: The $C A B G$ is usually the primary treatment strategy for symptomatic anomalous right coronary artery (ARCA). In ARCA, the ligation of the proximal RCA after CABG improves the diastolic flow in the graft and the pulsation index.

No conflict of interest 
338

\title{
SURGICAL ASPECTS
}

\section{LEFT VENTRICULAR PSEUDOANEURYSM FOLLOWING INFERIOR MYOCARDIAL INFARCTION}

\author{
L. Reija Lopez, A. Grand Bauza, J.M. Martinez Cereijo, A. Narvaez Muñoz, B. Adrio Nazar, \\ J. Garcia Carro, A. Fernández González, J. Sierra Quiroga, D. Duran Muñoz \\ Universitary Hospital Santiago de Compostela, Cirugía Cardíaca, Santiago de Compostela, Spain
}

Aims: Differential diagnosis between true and false aneurysm is especially difficult but essential due to the therapeutic and prognostic implications. The need improvements in cardiac imaging modalities for ensuring appropriate management of these patients

Method: Discussion of a case

Results: We report the case of a patient with a history of coronary artery disease who presented an inferior myocardial infarction with PTCA in the right coronary artery with distal flow TIMI III and post-MI pericarditis. He came to the emergencyl without elevation of myocardial necrosis markers but echocardiographic assessment revealed severe left ventricular systolic dysfunction and inferoposterior aneurysm/pseudoaneurysm, with intense auto-contrast and a sessile thrombus inside of the cavity, moderate mitral insufficiency and mild pericardial efusión. A cardiac magnetic resonance was performed to clarify the anatomy and diagnosis, the wall of this cavity demonstrated full-thickness late gadolinium enhancement, while the adjacent myocardium in the rest of the inferior and inferolateral wall returned a normal nulled signal (compatible with pseudo aneurysm), urgent surgery was indicated. In OR we resected the pseudoaneurysm and we needed to repair the ventricle with an endocardial patch of glutaraldehyde-fixed bovine pericardium using the same methods as those used in repairing true ventricular aneurysms.

Conclusion: Early myocardial revascularization leads to decrease the incidence of mechanical complications after myocardial infarction. Left ventricular pseudoaneurysm (LVP) is a rare and potentially lethal complication of acute myocardial infarction. True aneurysms tend to be managed conservatively whereas false aneurysms, due to the risk of rupture, are usually treated under urgent surgery

No conflict of interest 
339

\title{
SURGICAL ASPECTS
}

\section{TWO CASES OF VENTRICULOCORONARY CONNECTION FROM THE LEFT VENTRICLE IN HYPOPLASTIC LEFT HEART SYNDROME}

 \\ ${ }_{1}^{1}$ Gunma University Graduate School of Medicine, Pediatrics, Maebashi, Japan \\ ${ }^{2}$ Gunma Children's Medical Center, Cardiology, Shibukawa, Japan \\ ${ }^{3}$ Gunma Children's Medical Center, Cardiovascular Surgery, Shibukawa, Japan
}

Aims: We evaluate the treatment strategies for the presence of ventriculocoronary connections (VCCs) that is a risk factor for mortality after surgical palliation in patients with hypoplastic left heart syndrome (HLHS).

Method: We retrospectively reviewed 2 cases of VCC, both operative and non-operative cases.

Results: Case $1 \mathrm{~A}$ male newborn was diagnosed mitral stenosis and aortic atresia (MS/AA) type HLHS during his mother's pregnancy. Bilateral pulmonary banding (bPAB) was performed on the seventh day after birth. Cardiac catheterization 3 months after banding revealed VCC and the family did not agree with further interventions because of the higher risk of the Norwood procedure for HLHS with VCCs. The patient died of cardiac failure 865 days after bPAB. Case 2 A male newborn was detected MS/AA type HLHS with VCC antenatally. Three days after birth, bPAB was performed and the preoperative catheterization confirmed the presence of VCC. The Norwood procedure was performed without cardiac arrest in 2 month after banding. However the patient could not be weaned from cardiopulmonary bypass because of remarkable deterioration of the inferior wall motion of the left ventricle and finally died on the seventh postoperative day. On autopsy, the orifice of the left coronary artery was not detected. Numerous myocardial infarctions were seen in the left ventricle and even in the right ventricle.

Conclusion: Our case studies are valuable because no previous reports have described the natural course of HLHS after bPAB. If a patient can survive for 2 or more years, the opportunities for transplantation without palliation increase.

No conflict of interest 
340

\title{
SURGICAL ASPECTS
}

\section{WEEKEND OPERATING IS NOT ASSOCIATED WITH ADVERSE OUTCOMES IN CARDIAC SURGERY}

\author{
E. Caruana, S. Nashef
}

Papworth Hospital NHS Foundation Trust, Cardiothoracic Surgery, Cambridge, United Kingdom

\begin{abstract}
Aims: There is ongoing concern that procedures performed at weekends are associated with worse outcomes than those undertaken on weekdays. We sought to evaluate the impact of weekend operating at a UK-based specialist cardiac surgical centre, on length of stay of stay and mortality.
\end{abstract}

Method: Prospectively-collected data was obtained for all patients who underwent cardiac surgery at our institution between January and December 2013. Student $t$ test and Pearson's Chisquared test were used for statistical analyses.

Results: A total of 139 of $1,941(7.16 \%)$ procedures were performed at the weekend. The weekend case mix was similar to that of weekdays in terms of clinical urgency (weekday vs weekend, 71.2 vs $71.9 \%$ elective cases, $p=0.852$ ). There was no difference in logistic EuroSCORE (9.65 \pm 12.07 vs $10.79 \pm 15.56, p=0.297)$ between cases undertaken on weekdays and weekends respectively. Length of hospital stay ( $10.5 \pm 7.9$ vs $9.6 \pm 7.3$ days, $p=0.203$ ), hospital ( 2.4 vs $0.7 \%, p=0.203)$, 90 -day (4.4 vs $2.2 \%, p=0.200)$ and one-year ( 6.9 vs $4.3 \%, p=$ $0.244)$ mortality was similar in both groups.

Conclusion: This study demonstrates that in our centre, weekend surgical outcomes are equivalent to those of operations performed during the working week.

No conflict of interest 
341

\title{
SURGICAL ASPECTS
}

\section{DELAYED VENTRICULAR FREE WALL RUPTURE COMPLICATING ACUTE INFERIOR WALL MYOCARDIAL INFARCTION WITH PRIMARY PERCUTANEOUS INTERVENTION}

\author{
R. Shi', L. Bai', P. Liu', Y. Wang ${ }^{1}$, J. Ren', C. Sun ${ }^{1}$, Y. Yan² \\ ${ }^{1}$ The First Affiliated Hospital of Xi'an Jiaotong University, Department of Cardiovascular Disease, \\ Xi'an, China \\ ${ }^{2}$ The First Affiliated Hospital of Xi'an Jiaotong University, Department of Cardiovascular Surgery, \\ Xi'an, China
}

Aims: Ventricular free wall rupture (VFWR) is a most lethal complication of acute myocardial infarction (AMI) and surgical repair remains the only therapeutic option improving survival. Although primary percutaneous intervention $(\mathrm{PCl})$ could decrease the mortality of VFWR, it may lead to myocardial hemorrhage or even VFWR in some cases. We experienced a case of delayed VFWR complicating inferior wall AMI after PCl.

Method: A 52-year old male patient was referred to the emergency room with chest pain for 6 hours, and diagnosed of inferior AMI. Emergent coronary angiogram showed a total thrombus occlusion of the right coronary artery (RCA) with normal left anterior descending and left circumflex coronary artery. A drug-eluting stent was deployed to the RCA. However, 24 hours later after $\mathrm{PCl}$, the patient presented with suddenly cardiogenic shock and an echocardiography revealed the LVFWR and cardiac tamponade (Figure 1).

Results: With the volume loading and inotropic support, an emergent surgical repair was underwent. After remove of $400 \mathrm{ml}$ clots, a nomacroscopic tear with blood oozing from the left ventricular free wall near the apex and septum was identified (Figure 2) and then repaired with glued patch covering (Figure 3). After 3-month follow-up, echocardiography demonstrated a normal LV size and an LVEF of $50 \%$ with inferolateral and septal akinesia. 

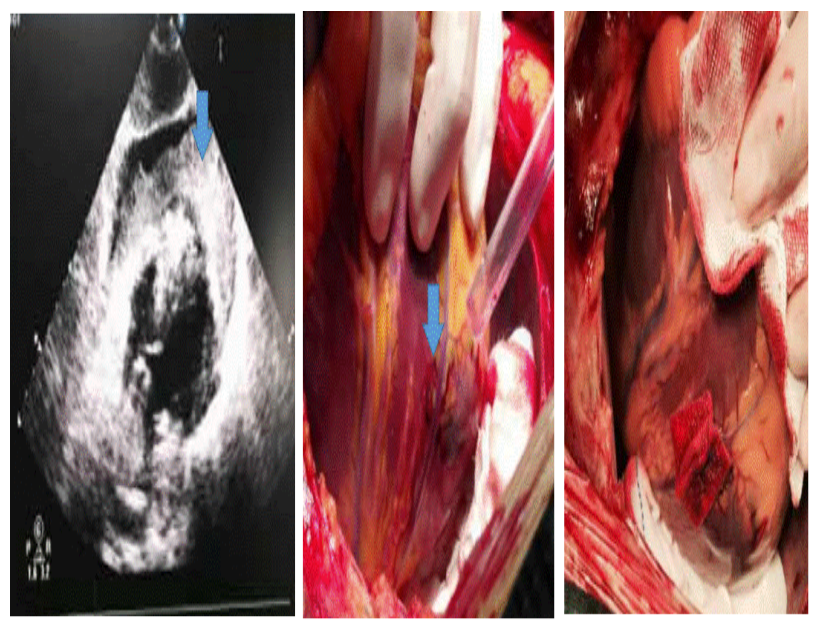

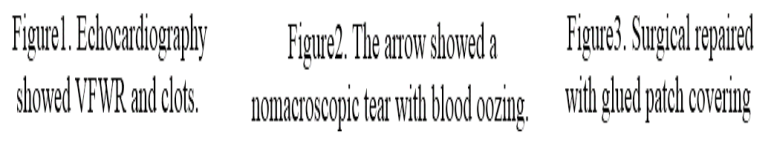

Conclusion: Considering the mechanical ventricular remodeling and reperfusion injury of AMI, a careful attention should be paid to the patient, even if previous $\mathrm{PCl}$ was successfully performed. Early diagnosis and surgical treatment of VWR are crucial for successful outcome.

No conflict of interest 
342

\title{
PROGNOSIS, PROGRESSION, PRACTICE PATTERNS
}

\section{CLINICAL OUTCOMES OF PATIENTS WITH STABLE CORONARY DISEASE ACCORDING TO ATHEROSCLEROTIC PLAQUE REMODELING}

\author{
S.U. Kwon \\ IIsan Paik Hospital - Inje University, Cardiac \& Vascular Center, Goyang, Korea
}

\begin{abstract}
Aims: Coronary multi-detector computed tomography has been shown to permit assessment of morphology and remodeling of coronary atherosclerotic lesion. Improved spatial and temporal image resolutions have facilitated coronary atherosclerotic plaque detection, characterization of plaque composition, and quantification of plaque burden. However, identification of lesions prone to cause acute ischemic events is difficult. We investigated whether atherosclerotic lesions detected by coronary CTA were predictive of acute coronary events.
\end{abstract}

Method: We analyzed 188 patients with stable angina who underwent 64-MDCT. Based on coronary artery remodeling index, the study population was divided into 2 groups: patients with negative and positive remodeling group. For advanced analysis of the coronary CTA data, color coded analysis was performed for a more detailed description which can be classified as lipid rich, fibro-necrotic, fibro-calcific, and calcific plaque.

Results: One cardiac death occurred because of fatal STEMI in positive remodeling group (PRG), and one with NSTEMI occurred in negative remodeling group (NRG). Nine patients $(21.4 \%)$ developed unstable angina with PRG and 10 patients $(6.9 \%)$ developed unstable angina in NRG $(p=0.016)$. A total of 11 patients $(7.5 \%)$ in NRG experienced the primary outcome as compared with 10 patients $(23.8 \%)$ in PRG $(p=0.009)$. In multivariate analysis, calcific plaque and minimal luminar area show decremental value $(\mathrm{HR}, 95 \% \mathrm{Cl} ; 0.01,0.00-0.55,0.01,0.00-0.051$; $p=0.027, p=0.023$, respectively).

Table 1 Predictors of cardiovascular events. Multivariate Cox proportional hazard regression analysis 


$\begin{array}{lccc}\text { Plaque characteristics } & \text { HR } & 95 \% \mathrm{Cl} & \mathrm{p} \text {-value } \\ \text { Fibronecrotic } & 4.73 & 0.15-153.8 & 0.382 \\ \text { Calcific } & 0.01 & 0.00-0.55 & 0.027 \\ \text { Spot calcium } & 0.89 & 0.32-2.49 & 0.821 \\ \text { Target lesion area } & 1.02 & 1.00-1.04 & 0.015 \\ \text { MLD } & 0.83 & 0.33-2.01 & 0.694 \\ \text { MLA } & 0.01 & 0.00-0.51 & 0.023 \\ \text { Lesion length } & 0.92 & 0.70-1.20 & 0.538 \\ \text { \% Area stenosis } & 0.87 & 0.74-1.02 & 0.090 \\ \text { \% diameter stenosis } & 1.04 & 0.96-1.14 & 0.334 \\ \text { Target lesion plaque burden } & 0.52 & 0.24-1.12 & 0.096 \\ \text { Target vessel volume } & 1.05 & 0.99-1.11 & 0.102 \\ \text { Lipid rich plaque volume } & 0.81 & 0.58-1.13 & 0.207 \\ \text { Fibrotic plaque volume } & 0.99 & 0.93-1.07 & 0.930\end{array}$

Conclusion: Plaque calcification and negative remodeling by coronary CTA provides decreased risk for subsequent acute coronary events.

No conflict of interest 
343

PROGNOSIS, PROGRESSION, PRACTICE PATTERNS

\section{CLINICAL OUTCOMES OF PERCUTANEOUS CARDIOPULMONARY SUPPORT IN CARDIAC OR RESPIRATORY FAILURE: COMPARISONS OF CARDIAC VERSUS NON-CARDIAC FAILURE}

S.U. Kwon

IIsan Paik Hospital - Inje University, Cardiac \& Vascular Center, Goyang, Korea

Aims: Percutaneous cardiopulmonary support is a widely accepted treatment for severe cardiopulmonary failure because this system can be rapidly applied in emergency situations. However, there is no available data on clinical outcome in patients between cardiac and noncardiac origin cardiopulmonary failure. We compared clinical outcomes of cardiac and noncardiac failure.

Method: We analyzed 61 consecutive patients with severe cardiopulmonary failure and complicating cardiogenic shock who were assisted by an emergent bypass system (EBS $\AA$ Terumo, Tokyo, Japan) between January 2012 and May 2015. The primary outcome was the success rate of weaning from EBS. The secondary outcome was in-hospital mortality.

Results: The mean duration of PCPS was 77.6 hours and that of cardiopulmonary resuscitation (CPR) was $32(52.5 \%)$. The rate of weaning was $23(37.7 \%)$ and the rate of weaning from cardiac group was higher than non-cardiac group $(51.2 \%$ vs. $10.0 \%, p=0.002)$. In-hospital mortality occurred for 45 patients (63.4\% vs. 95.0\%, $p=0.012$ ).

Fig. 1 Kaplan-Meier failure estimates curve. A comparison of survival between cardiac and noncardiac group

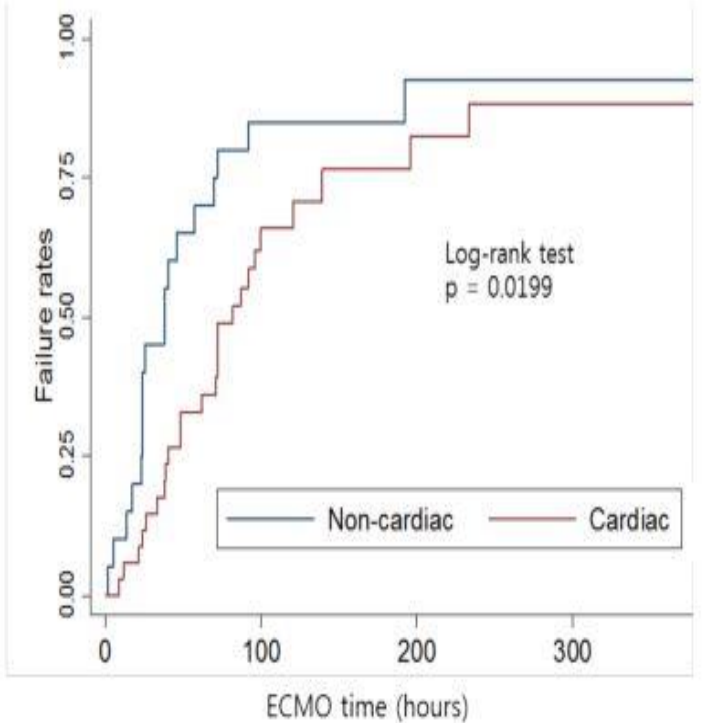


Conclusion: Cardiopulmonary failure with non-cardiac origin was associated with high mortality. An APACHE II score \& renal replacement therapy might serve as outcome for risk stratification.

No conflict of interest 
344

PROGNOSIS, PROGRESSION, PRACTICE PATTERNS

\title{
IMPROVING CARDIAC SURGERY OUTCOMES: A SECONDARY DATA ANALYSIS OF A CARDIAC SURGERY CLINCIAL PATHWAY
}

\author{
T. Harties ${ }^{1}$, J. Gilliam², W. Stinson 3 , C. Garvan², L. Cowan', A. Thompson² \\ ${ }^{1}$ University of Florida, Gainesville, USA \\ 2University of Florida, College of Nursing, Gainesville, USA \\ ${ }^{3}$ North Florida South Georgia VHA, Cardiothoracic Surgery, Gainesville, USA \\ ${ }^{4}$ North Florida South Georgia VHA, Research, Gainesville, USA
}

\begin{abstract}
Aims: The purpose of this IRB approved study was to improve patient outcomes in the cardiac surgery population within our intensive care unit (ICU). Outcomes were collected on length of stay (LOS), ventilation hours, and early patient mobilization. Our outcomes were poor, when compared to national benchmark data. Consulting the literature, we noted fast-tracking or rapid recovery practices were used in many facilities to standardize care and streamline processes. An interdisciplinary team was formed to create a Cardiac Surgery Care Pathway, to improve our patients' outcomes.
\end{abstract}

Method: After implementation of the care pathway, two cohorts of patients were evaluated and compared: Pre-Implementation and Post-Implementation. The first fifty patients from each cohort meeting the inclusion/exclusion criteria were enrolled. Twenty-eight demographic and/or outcomes variables were collected. Descriptive, Chi Square and T-Test statistics were utilized to describe and compare the sample characteristics, and clinical results between the pre and the post-pathway cohorts using SAS Software.

Results: Several variables were noted to be statistically significant between the Pre and PostPathway groups, with a $p=<0.01$. They included: 1 ) reduced time on ventilator, 2) early patient mobilization out of bed, 3) patients mobilized OOB with invasive lines, 4) reduced SICU LOS, 5) reduced total hospital LOS.

Conclusion: The post implementation outcome results reflected our patients as and the lowest in post-operative mortality, and 3rd lowest in the nation for extubation times. Overall, results demonstrate a significant improvement in patient outcomes without a difference in patient disposition from pre to post-pathway $p=1.000$; reflecting a $\$ 500,000$ savings in the first six months.

No conflict of interest 
345

PROGNOSIS, PROGRESSION, PRACTICE PATTERNS

\title{
THE PROGNOSTIC IMPORTANCE OF OBJECTIVE NUTRITIONAL INDEXES IN OLDER PATIENTS WITH ST-ELEVATION MYOCARDIAL INFARCTION UNDERGOING PRIMARY CORONARY INTERVENTION
}

\author{
A. Mazzone ${ }^{1}$, G. Basta', K. Chatzianagnostou', U. Paradossi', N. Botto ${ }^{1}$, S. Del Turco'2, \\ A. Taddei' ${ }^{2}$ S. Berti ${ }^{1}$ \\ ${ }^{1}$ Heart Hospital - Fondazione Toscana Gabriele Monasterio, Adult Cardiac /Cardiosurgery Dep., \\ Massa, Italy \\ ${ }^{2}$ Institute of Clinical Physiology, Consiglio Nazionale delle Ricerche CNR, Pisa, Italy
}

Aims: The optimal treatment strategies for acute ST-elevation myocardial infarction (STEMI) in older patients are unclear because of the high risk of mortality in this population. Hypoalbuminemia has been reported to worsen the prognosis of some older patients, but the prognostic impact of nutritional status in patients after STEMI is poorly understood.

Method: We investigated the impact of nutrition, by using the controlling nutritional status (CONUT) score and the prognostic nutritional index (PNI) score on outcomes of patients with acute STEMI.

We examined prospectively 945 consecutive STEMI patients (706 men) undergoing primary $\mathrm{PCl}$ and stent implantation. The CONUT score was calculated by the serum levels of albumin, total cholesterol, and lymphocyte count. The PNI score by albumin and lymphocyte count.

Results: The patients were divided in two groups according to the age cut off point, " $\geq 71$ year" and " $<71$ years" ( 335 pts of $78.5 \pm 5.4$ years and 610 pts of $57.9 \pm 8.5$ years, respectively). During a median follow-up of 2 years, $14.9 \%$ of patients in the group " $\geq 71$ years" and $1.1 \%$ in the group " $<71$ years" died for all-cause of death. In the group " $\geq 71$ years" at univariate analysis, apart from the traditional prognostic factors, the CONUT score $(O R=1.34, p<0.0001)$ and the PNI score $(O R$ $=0.89 \mathrm{p}<0.001$ ) were also associated with increased mortality. The multivariate Cox model, revealed that BNP was associated with mortality [HR=1.97 (95\% $\mathrm{Cl} 1.2-3.3), p=0.009]$.

Conclusion: In STEMI patients, the nutritional status, in addition to other comorbidities, can affect the prognosis in elderly patients but not in younger patients.

No conflict of interest 
346

PROGNOSIS, PROGRESSION, PRACTICE PATTERNS

\section{MITRAL VALVE PROLAPSE AND CEREBRAL ISCHEMIA: NURSE SPECIALIST EXPERIENCE IN ECHOCARDIOGRAPHY}

\section{S. Vucinic 1 , D. Sagic ${ }^{2}$, V. Perovic ${ }^{2}$}

${ }_{1}^{1}$ Institute of Cardiovascular Diseases, Echocardiography, Belgrade, Serbia

2Institute of Cardiovascular Diseases, Cath Lab, Belgrade, Serbia

Aims: Mitral valve prolapse (MVP), with $2 \%$ general population prevalence (more frequent in younger women) is bening condition. Although rare, this patients can experience serious complications, such as sudden cardiac death $(0,5-2 \%)$ and stroke $(0,6 \%)$.

The aim of this stydi was to identify the prognostic value of a group of risk factors that were obtained by combining non-invasive methods (echocardiography and holter-ECG) in assesment of risk for stroke (embolic/ischemic) in subjects with non-complicated MVR.

Method: There were 158 subjects examined in the period 2014. in ultrasound lab. They were divided into two groups. In the first group there were 40 subjects aged $20-45$ (36\% male, 64\% female) with clearly identified MVP without complications. In the second group there were 87 subjects aged $37-50$ (22\% male, $78 \%$ female) with clearly identified MVP without complications who suffered stroke in observed period.

Results: We determined by using statistical methods that a group of risk factors: anterior mitral leaflet thickness-AMLt $(7,15 \pm 1,2 \mathrm{~mm} ; \mathrm{p}<0,05$; HR 5,7), interatrial septel aneurysm ( $p<0,05 ; \mathrm{HP}$ $9,6)$, paroxysmal atrial fibrillation ( $p<0,05 ; \mathrm{HR} 8,7$ ), left atrium systolic volume-LAVs $(42,6 \pm 10,7, p<0,05 ; \mathrm{HR} 3,8)$ and asystolic pause $(3,5 \pm 1 \mathrm{~s} ; \mathrm{p}<0,05$, HR 5,2$)$ defined by a combination of non-invasive methods can predict stroke onset with a $87 \%$ sensitivity, in patients with MNP.

Conclusion: This study confirmed that by combining non-invasive methods we can create a group of risk factors with a $87 \%$ prognostic sensitivity for onset of stroke, extremely rare $(1 / 6000$ patients per year) complication.

No conflict of interest 
347

ANGINA PECTORIS, UNSTABLE ANGINA, ACS

\title{
DESIGN AND RATIONALE FOR RUSSIAN NATIONAL REGISTRY OF ACUTE CORONARY SYNDROME TREATMENT AND APPROACH IN DUAL ANTIPLATELET THERAPY
}

\author{
O. Apanova ${ }^{1}$, E. Oschepkova², V. Dmitriev² \\ ${ }^{1}$ AstraZeneca Russia, Medical, Moscow, Russia \\ ${ }^{2}$ Russian Cardiology Research and Production Centre, \\ Department of the Cardiovascular Diseases Registries, Moscow, Russia
}

\begin{abstract}
Aims: There is no data on long-term outcomes for post-acute coronary syndrome (ACS) patients in Russia such as cardiovascular (CV) mortality, recurrent myocardial infarction (MI), stroke, hospitalizations associated with CV diseases, and revascularizations. Very few data on duration of dual antiplatelet therapy in post-ACS Russian patients after hospital discharge are available. Existing Russian ACS registries collect only hospital data.

The primary objectives of the STREAM registry are to understand the short-term antithrombotic management approach in a real-life hospital setting for ACS patients on the base of a retrospective data, and to describe the long-term ( 2 years) prognosis in a prospective arm that includes patients with ACS taking ticagrelor after hospital discharge.
\end{abstract}

Method: STREAM registry is a multicenter, non-interventional, descriptive, cross-sectional study, which consists of two parts. The retrospective part will include data of 10,000 patients that have already been enrolled in the ACS Registry. The prospective part is a single-arm, longitudinal cohort study, which will include 1,000 consecutive patients with ACS who will be discharged from hospital with ticagrelor prescription. A descriptive analysis approach will be used to analyze study objectives.

Results: Patients will be recruited by the specialists working in the hospitals participating in the Russian National ACS Register, which provides information concerning characteristics and treatment of ACS patients in 167 hospitals from the 46 regions of the Russian Federation.

Conclusion: The current study will provide information about the current in-hospital treatment of ACS and the long-term (up to 2 years) prognosis in Russian patients with ACS on treatment with ticagrelor in real-life setting.

No conflict of interest 
348

ANGINA PECTORIS, UNSTABLE ANGINA, ACS

\title{
IT IS NOT MANDATORY TO USE TRIPLE RATHER THAN DUAL ANTI-PLATELET THERAPY AFTER CORONARY INTERVENTION WITH A SECOND-GENERATION DRUG-ELUTING STENT
}

\author{
Y.S. Choi, K. Ami, P. Chul-Soo \\ Catholic University of Korea Yeouido St.Mary's Hospital, Internal Medicine, Seoul, Korea
}

Aims: Research has shown that triple antiplatelet therapy with cilostazol results in better clinical outcomes than dual antiplatelet therapy in patients treated with a first-generation drug-eluting stent (DES); however, it is unclear whether triple antiplatelet therapy has a similar efficacy after the implantation of second-generation DES.

Method: In the COACT (CathOlic medical center percutAneous Coronary inTervention) registry, 1248 study subjects with coronary artery disease (CAD) who underwent PCI with an everolimusor zotarolimus-eluting stent (Endeavor, Xience V or Promus) were analyzed. The patients were divided into the following two groups after propensity score matching( $n=724 ; M=422(58.3 \%)$; mean age $=66.1 \pm 11.0$ years): Group 1: patients treated with dual antiplatelet drugs (aspirin and clopidogrel; $n=362 ; M=213$ (58.8\%); mean age=65.6 \pm 11.7 years);Group 2: patients treated with triple antiplatelet drugs (aspirin, clopidogrel and cilostazol; $n=362 ; M=209$ (57.7\%); mean age $=65.6 \pm 11.7$ years). The incidences of clinical outcomes were compared between the two groups

Results: The mean follow-up duration was $13 \pm 10$ months, and the cumulative incidence of MACE was $6.3 \%$ in Group 1 and $7.7 \%$ in Group 2. There were no significant differences in MACE (death, non-fatal MI and stroke) between the two groups (OR, 1.210; 95\% Cl, 0.772-1.898; $\mathrm{p}=0.406$ ). Kaplan-Meier curves for MACE did not show any survival benefit for triple anti-platelet therapy, even in patients with acute coronary syndrome.

Conclusion: In patients treated with a second-generation DES implantation, there is no additive clinical benefit to using triple rather than dual antiplatelet therapy

No conflict of interest 
349

ANGINA PECTORIS, UNSTABLE ANGINA, ACS

\title{
PREVALENCE, THERAPEUTIC MANAGEMENT AND MEDIUM-TERM PROGNOSIS OF SPONTANEOUS CORONARY ARTERY DISSECTION: RESULTS FROM A DATABASE OF 5350 PATIENTS
}

\author{
C. Graidis ${ }^{1}, \underline{\text { D. Dimitriadis }}{ }^{1}$, G. Dimitriadis ${ }^{1}$, G. Tsonis ${ }^{2}$, V. Karasavvidis ${ }^{1}$, T. Bitsis ${ }^{1}$, \\ E. Argyropoulou ${ }^{1}$, A. Antoniou ${ }^{3}$, G. Karakostas ${ }^{4}$ \\ ${ }^{1}$ Euromedica Kyanous Stavros General Hospital, Interventional Cardiology, Thessaloniki, Greece \\ 'Euromedica Kyanous Stavros General Hospital, Cardiothoracic Department, Thessaloniki, \\ Greece \\ ${ }^{3}$ General Hospital of Serres, Cardiology Department, Serres, Greece \\ ${ }^{4}$ General Hospital of Kilkis, Cardiology Department, Kilkis, Greece
}

\begin{abstract}
Aims: To assess the prevalence, clinical presentation, therapeutic management and mediumterm prognosis of spontaneous coronary artery dissection (SCAD).

Method: A retrospective single-center cohort study. Over a 7-year period in 5350 coronary angiographies study identified 9 patients with angiographically confirmed SCAD $(0.17 \%)$. Mean age was 42.6 years and $88,9 \%$ were female. Most patients presented with an acute myocardial infarction (8 pts). SCD had a diffuse angiographic pattern in most cases.
\end{abstract}

Results: Coronary angiography revealed involvement of coronary vessel distribution: LAD in 7 cases $(77,8 \%), R C A$ in one case $(11,1 \%)$ and LM in another $(11,1 \%) .8$ cases were treated with coronary stenting and 1 patient underwent coronary artery bypass grafting. The mortality rate was $0 \%$ during hospitalization. In one patient we dealt with retrograde extension of the dissection to the left main as an complication. He was treated with $\mathrm{PCl}$ in day 5 of his hospitalization. During follow-up no cases of repeated SCAD were recorded and 1 case of repeat percutaneous coronary intervention due to restenosis.

Conclusion: SCAD affects a young, predominantly female population, frequently presenting as ST elevation myocardial infarction ECG pattern. Revascularisation of SCAD patients might be challenging, with no guidelines yet in place. In the case where $\mathrm{PCl}$ is required, the potential benefits and use of bioabsorbable stent should be considered. Especially in cases of extensive or distally located dissection.

No conflict of interest 
350

ANGINA PECTORIS, UNSTABLE ANGINA, ACS

\title{
TAKO-TSUBO ACUTE SYNDROME AS A CAUSE OF ACUTE CORONARY SYNDROME WITH NORMAL CORONARY ARTERIES. A CASE REPORT
}

\author{
E. Garcia ${ }^{1}$, A. Rodriguez-Bermejo², V. Bonilla' ${ }^{1}$, J.A. Garcia Lledó ${ }^{1}$ \\ ${ }^{1}$ Hospital Universitario Principe de Asturias, Cardiology department, Alcala de Henares, Spain \\ ${ }^{2}$ Hospital Universitario Principe de Asturias, Primary Care, Alcala de Henares, Spain
}

Aims: Up to a $12 \%$ of ACS doesn't show significant stenosis at the angiography. This incidence is higher in young women

Method: We report the case of a patient who was hospitalized due to an Acute Coronary Syndrome with normal coronary arteries

Results: 58-year-old women, ex-smoker, presents to the ER department with sudden dysnea that occurred after an argument. ECG shows Sinus Tachycardia with poor growth of $\mathrm{R}$ in precordial leads and flattened T from V1 to V6. Peak Troponine I 11 and CK 400.

Echocardiography reveals apical akinesia and mildly lowered systolic function. No significant coronary stenosis was found in the angiography. Ventriculography shows apical akinesia and a thrombus in apex. During hospitalization no changes were seen in ECG or echocardiography. A month later echocardiography was completely normal, with also normal hearth MRI. The compatibility of the clinical course leads to the diagnosis of Tako-Tsubo Syndrome 



Conclusion: Tako-Tsubo Syndrome or apical ballooning syndrome is a reversible miocardiopathy that is more frequent in post-menopausal women after emotional stress. Clinic and ECG may be undistinguishable from a myocardial infraction, but with a lower elevation of enzymes. Apical akinesia is the typical form, but atypical cases with dysfunction of mid and basal regions have also been reported. Intracavitary thrombus appear in a 1-2\%. After the acute phase, clinical course is usually benign, with an annual recurrency of $1.5 \%$

No conflict of interest 
351

ANGINA PECTORIS, UNSTABLE ANGINA, ACS

\section{TWO CASES OF HARD PCI FOR SEVERE LOOP-SHAPED RCA WITH AMI}

S. Ito, Y. Hatano, T. Amemiya, M. Hashimoto, H. Suesada

Nishitokyo Chuo General Hospital, Cardiology, Tokyo, Japan

Aims: We experienced two cases of sever loop shaped RCA with AMI.

Method: We performed PCI for two cases of AMI.

Results: A 69-year-old man admitted to our hospital with chest pain. An Inferior acute myocardial infarction (AMI) was detected on the electrocardiogram (EKG). A coronary artery angiogram (CAG) revealed a total occlusion in the distal right coronary artery (RCA). We performed thrombectomy, however we could not cross the lesion due to sever tortuosity of proximal RCA. A semi compliance balloon successfully crossed the lesion. After ballooning, the blood flow of RCA was delayed because a severe loop-shaped in the distal RCA was suspected to exist. We successfully deployed bare metal stent (BMS) under a grand slam wire. Three days after $\mathrm{PCl}$, a sub-acute stent thrombosis occurred. An emergency CAG revealed a total occlusion at the BMS. Unfortunately, we performed unsuccessful PCI. Then an emergency CABG was successfully performed.

An 84-year-old man with chest pain. An inferior AMI was detected on the EKG. A CAG revealed a total occlusion in the mid RCA. We performed PCI for the RCA. A thrombectomy catheter could not cross the lesion. After ballooning, the blood flow of RCA was delayed. Because a severe loop-shaped in the mid RCA was suspected to exist. We successfully deployed drug eluting stents without a grand slam wire because proximal RCA was no tortuosity.

Conclusion: It is necessary to consider the both characteristics of the lesion and the patient in order to select the kinds of stent for the severe loop-shaped coronary lesion.

No conflict of interest 
352

ANGINA PECTORIS, UNSTABLE ANGINA, ACS

\title{
PREVIOUS CEREBROVASCULAR DISEASE IS THE MOST IMPORTANT INDEPENDENT PREDICTOR OF CLINICAL OUTCOMES IN ELDERLY PATIENTS WHO UNDERGO PERCUTANEOUS CORONARY INTERVENTIONS
}

\author{
Y.H. Kim, H. Ae-Young \\ Kangwon National University, Schoo of Medicine- Division of Cardiology- \\ Department of Internal Medicine, Chuncheon-Si- Gangwon-Do, Korea
}

Aims: To determine the most important independent risk factor that could predict clinical outcomes in elderly patients who undergo PCI.

Method: A total of 1884 consecutive patients who underwent PCI were evaluated. The enrolled patients were divided into 2 groups according to age, namely, younger patients who were aged < 75 years and elderly patients who were aged $\geq 75$ years. The study's primary end point was the occurrence of major adverse cardiac or cerebrovascular events (MACCE) at 1 year

Results: The 1-year cumulative incidence of MACCE was $4.9 \%$, and it was $3.8 \%$ in the younger patient group and $9.9 \%$ in the elderly patient group $(P<0.001)$. Forty-nine patients $(2.6 \%)$ died during the follow-up period. The 1-year mortality rate was $1.5 \%$ (23 patients) for the younger patient group and $7.1 \%$ (26 patients) for the elderly patient group $(P<0.001)$. Previous cerebrovascular disease was significantly correlated with MACCE at 1 year in elderly patients treated with $\mathrm{PCl}(\mathrm{HR}, 3.079 ; 95 \% \mathrm{Cl}, 1.276-7.426, \mathrm{P}=0.012)$.

Conclusion: Previous cerebrovascular disease is the most important independent predictor of the occurrence of MACCE in elderly patients 1 year after $\mathrm{PCl}$

No conflict of interest 
353

ANGINA PECTORIS, UNSTABLE ANGINA, ACS

\title{
ANXIETY IS ASSOCIATED WITH A CIRCULATING PRO-INFLAMMATORY CELLULAR IMBALANCE IN PATIENTS WITH ACUTE CORONARY SYNDROMES
}

\author{
A. Mazzone ${ }^{1}$, M. Marotta 1 , S. Sbrana ${ }^{2}$, N. Botto ${ }^{3}$, U. Paradossi ${ }^{1}$, A. Taddei ${ }^{4}$, C. Palmieri ${ }^{5}$, S. Berti ${ }^{5}$ \\ ${ }^{1}$ Heart Hospital - Fondazione Toscana Gabriele Monasterio, Adult Cardiac /Cardiosurgery Dep., \\ Massa, Italy \\ ${ }^{2}$ Institute of Clinical Phisiology- CNR - Pisa and Massa- Italy, Biochemical Chemistry Laboratory, \\ Massa, Italy \\ ${ }^{3}$ Heart Hospital - Fondazione Toscana Gabriele Monasterio, Biochemical Chemistry Laboratory, \\ Massa, Italy \\ ${ }^{4}$ Institute of Clinical Phisiology- CNR - Pisa and Massa- Italy, Statistical Laboratory, Massa, Italy \\ ${ }^{5}$ Heart Hospital - Fondazione Toscana Gabriele Monasterio, Haemodinamic laboratory, Massa, \\ Italy
}

Aims: Previous experimental and clinical studies clearly confirmed the presence of a stressinduced immune dysregulation. Anxiety and depression, in particular, modify the normal balance of inflammation and coagulation markers, and are associated with cardiovascular disease. The aim of our study has been to investigate the association of anxiety and depression with cardiovascular prognostic hematological and biochemical markers in patients with different settings of Acute Coronary Syndromes (ACS).

Method: We evaluated 442 consecutive subjects (Males $=$ n.309, mean age $66.5 \pm 11.84$ ), admitted to our Cardiology Unit for ACS (STEMI, NSTEMI, Unstable Angina, Takotsubo disease). The Hospital Anxiety and Depression Scale (HADS-A; HADS-D) was used to assess non-somatic symptoms of anxiety and depression, just before patient care discharge. The anxious and depressed subjects were identified by HADS-A and HADS-D scores ${ }^{3} 9$. We examinated admission routine peripheral blood laboratory parameters including high-sensitivity C-Reactive Protein (hs CRP), ESR, fibrinogen, platelets, differential white cell count and troponin peak.

Results: Unpaired t-test statistical analysis showed significant associations of a HADS -A ${ }^{3} 9$ with Platelet/Lymphocyte $(p=0.0087)$ and Neutrophil/Lymphocyte Ratios $(p=0.0184)$. In particular, Neutrophil/Lymphocyte ratio correlated with troponin peak levels $(p=0.0069)$. Interestingly, females showed, at the Chi Squares statistical analysis, significantly higher HADS-A and HADS$\mathrm{D}(p=0.007$ and $p=0.0009$, respectively).

No association was found between HADS-D and cellular pro-inflammatory markers.

Conclusion: Our data indicate that anxious individuals, but not depressed subjects, have higher circulating ratios of pro-inflammatory cellular subsets known to predict major adverse outcomes in various clinical setting of ACS.

No conflict of interest 
354

ANGINA PECTORIS, UNSTABLE ANGINA, ACS

\title{
RISK FACTORS FOR CORONARY HEART DISEASE IN RHEUMATOID ARTHRITIS
}

\author{
M. Parvu' ${ }^{1}$ L.C. Adascalitei ${ }^{2}$, S. Coman ${ }^{3}$ \\ ${ }^{1}$ Tirgu Mures Emergency Clinical County Hospital- \\ University of Medicine and Pharmacy Tirgu Mures, Rheumaology, Tirgu Mures, Romania \\ ${ }^{2}$ Tirgu Mures Emergency Clinical County Hospital, Intensive care, Tirgu Mures, Romania \\ ${ }^{3}$ Tirgu Mures Emergency Clinical County Hospital, Cardiology, Tirgu Mures, Romania
}

In rheumatoid arthritis (RA) patients have been shown to have an increased risk to coronary heart disease (CHD) and premature atherosclerosis (AS).

Objective: The purpose of the study was to evaluate the factors involved in cardiovascular risk (CHD and AS) in patient with RA.

Material and method: $113 \mathrm{RA}$ patients were retrospectively analyzed. All patients were diagnosed according to ACR/EULAR 2010 criteria. Atherosclerosis has been evaluated using the carotid intimae-media thickness (IMT), which was measured at the common carotid artery, using B-mode ultrasound. Coronary heart disease was evaluated at ECG recorded and medical chart. Cardiovascular risk was calculated using Framingham Score (FS). Laboratory evaluation included antinuclear antibodies (ANA), anticardiolipin antibodies (ACA), anticyclic citrullinated peptide antibodies (CCP) by ELISA method.

Results: $17 \%$ of the patients were male and $83 \%$ were female, mean age $54 \pm 11,76$ SD (years), mean disease duration of $6,23 \pm 5,14 \mathrm{SD}$ (years). Antibodies positivity was identified as following: $88 \%$ for CCP, $30 \%$ for ACA, $36,8 \%$ for ANA. IMT mean value $(\mathrm{mm})$ was $0,84 \pm 0,19 \mathrm{SD}$. We found correlations between traditional risk factors (quantified by SF) in predictions IMT index with $r=0,70$ $(r 2=0,49)$, while for $S F$ was $r=0,84(r 2=0,74)$. Antibodies were correlated with subclinical atherosclerosis and coronary heart disease, as following: for ANA $p=0.001$ and for ACA $p=0,007$ but not for CCP $\mathrm{p}=0,15$.

Conclusions: In patients with RA, ANA and ACA antibodies measurement must be emphasised, since it seems that they can be used in predictive estimate of coronary heart disease. Traditional risk factors are important to be evaluating for predicting cardiovascular risk.

No conflict of interest 
355

ANGINA PECTORIS, UNSTABLE ANGINA, ACS

\section{VIRTUAL 5-FRENCH INTRA-AORTIC PUMPING WITH THE GLIDE SHEATH SLENDER AND 6-FRENCH INTRA AORTIC BALLOON CATHETER}

N. Taniguchi, A. Takahashi, T. Hata, S. Nakajima, Y. Mizuguchi, T. Yamada, S. Hashimoto Sakurakai Takahashi Hospital, Cardiology, Kobe, Japan

Aims: The aim of this study is to evaluate the feasibility and safety of the use of 6-Fr Glidesheath Slender, having thinner shaft, for a 6 -Fr intra-aortic balloon pump (IABP) catheter.

Method: From August 2014 to March 2015, 24 patients with acute coronary syndrome underwent $\mathrm{PCl}$ with support with a 6-Fr IABP catheter (Zeon Medical Corp., Tokyo, Japan) through a 6-Fr Glidesheath slender. The adverse events, including access site complications, the kinking of the sheath during procedure, any kind of balloon pumping failures were retrospectively investigated.

Results: Insertion of the IABP catheters was successful for all patients. The mean support time was $32.4 \pm 22.0 \mathrm{~h}$. No hemorrhagic event nor severe limb ischemia was observed. The kinking of the shaft occurred during the insertion procedure in one patient, however, subsequent balloon pumping was well maintained and did not require exchange of the sheath or IABP catheter. The any kind of IABP balloon pumping failure were not observed.

Conclusion: The use of the Glide sheath slender is feasible for the insertion of 6-Fr IABP catheter and may reduce vascular complications in patients who have undergone $\mathrm{PCl}$, although this sheath is made for the dedicated use of radial access.

No conflict of interest 
356

ANGINA PECTORIS, UNSTABLE ANGINA, ACS

\title{
EFFECT OF INVASIVE CORONARY ANGIOGRAPHY ON SURVIVAL TO 12 MONTHS IN TROPONIN-NEGATIVE NSTEACS (TN-NSTEACS)
}

 \\ V. Sundararajan ${ }^{1}$ \\ ${ }^{1}$ The University of Melbourne, St Vincent's Hospital- Department of Medicine, Melbourne, \\ Australia \\ ${ }^{2}$ Monash University and Monash Health, Monash Heart and Monash Cardiovascular Centre, \\ Melbourne, Australia \\ ${ }^{3}$ St Vincent's Hospital Melbourne- The University of Melbourne, \\ Department of Cardiology and Department of Medicine, Melbourne, Australia \\ ${ }^{4}$ The University of Melbourne, Melbourne Institute of Applied Economic and Social Research, \\ Melbourne, Australia \\ ${ }^{5}$ Victorian Department of Health, Department of Health, Melbourne, Australia
}

Aims: To assess the impact of angiography on 12 month mortality after TN-NSTEACS.

Method: Retrospective cohort study using linked, coded hospital data from Victoria, Australia (population 5mil), 2001-2011. Treated and untreated groups propensity-score matched prior to logistic regression controlling for additional invasive coronary angiography (ICA); deterministic sensitivity analysis performed to test the robustness of results to residual confounding from unmeasured factors.

Results: N=54,198 TN-NSTEACS admitted emergently to angiography hospitals and surviving $>=48 \mathrm{hrs} ; 39.5 \%$ underwent angiography. No significant covariate differences for $n=12,265$ propensity-score-matched pairs. Mortality was the highest within the first 3 months (1.8\%). No effect of angiography on survival to hospital discharge (odds ratio (OR) 1.22, 95\% confidence interval $(\mathrm{Cl}) 0.88,1.70)$. However, of $n=12194$ pairs discharged alive, angiography was associated with increased 3-month survival (OR 2.57, 95\% CI 2.05, 3.23). Sensitivity testing for the effect of an unmeasured confounder (relative risk 1.5, prevalence $30 \%$ and $80 \%$ in non-ICA and ICA groups respectively) did not alter results. The effect of angiography persisted at 6 months (OR 2.28, 95\% $\mathrm{Cl} 1.72,3.02$ ) and at 12 months (OR 2.29, 95\% Cl 1.83, 2.87).

Conclusion: Patients with TN-NSTEACS benefit from ICA, with improved survival to 12 months.

No conflict of interest 
357

ANGINA PECTORIS, UNSTABLE ANGINA, ACS

\title{
EFFECT OF THE KV1.3 VOLTAGE-GATED POTASSIUM CHANNEL BLOCKER PAP-1 ON THE INITIATION AND PROGRESS OF ATHEROSCLEROSIS IN A RAT MODEL
}

\author{
W. Xiaofen, R. Xu, M. Cao, L. Ruan, X. Wang, C. Zhang \\ Department of Gerontology, Tongji Hospital, Tongji Medical College, \\ Huazhong University of Science and Technology, Wuhan, China
}

Acute coronary syndrome is a serious medical emergency. It occurs when an atherosclerotic plaque ruptures, leading to thrombus formation within a coronary artery. Previous studies have shown that $\mathrm{T}$ cells are involved in the initiation and progression of acute coronary syndrome.

CD4+CD28null T lymphocytes increase in atherosclerotic plaque, and voltage-gated potassium channel Kv1.3 blockers can suppress the function of these cells in vitro by preventing exocytosis of their cytoplasmic granules. The purpose of this study was to investigate the effect of PAP-1, a small molecule voltage-gated potassium channel Kv1.3 blocker, on the development of atherosclerosis (AS) in a rat model and the potential mechanism for this effect. Plasma lipids, interferonc, CRP, CD4+CD28null T cells, and perforin were increased and unstable atherosclerotic plaques developed in the rat model of AS. Blockade of the Kv1.3 potassium channel via PAP-1 administration decreased perforin levels and prevented plaque formation but had no effect on the other changes seen in this AS model. These findings suggest that the small molecule, voltage-gated potassium channel Kv1.3 blocker PAP-1 can suppress the development of AS in a rat model, most likely by inhibiting the exocytosis of cytoplasmic granules from CD4+CD28null T cells.

Document not received 
358

ANGINA PECTORIS, UNSTABLE ANGINA, ACS

\title{
ANGIOPLASTY IMPROVES MORTALITY IN THE MANAGEMENT OF ACUTE CORONARY SYNDROME IN PATIENTS WITH CHRONIC ANAEMIA
}

\author{
M. Zaman 1 , R.S. Gorantla², H. Uppal', N. Lavu', S. Chandran ${ }^{4}$, R. Potluri ${ }^{3}$ \\ ${ }^{1}$ University Hospital of South Manchester, Cardiology, Manchester, United Kingdom \\ ${ }^{2}$ Columbia University of Physicians and Surgeons- Bassett Medical Center, Internal Medicine, \\ New York, USA \\ ${ }^{3}$ Aston University, Acalm Study Unit in Collobaration with Aston Medical School, Birmingham, \\ United Kingdom \\ ${ }^{4}$ North Western Deanery, Acute Medicine, Manchester, United Kingdom
}

\begin{abstract}
Aims: Angioplasty has changed the management of acute coronary syndromes (ACS). However, in patients with chronic anaemia the evidence for angioplasty in ACS is less well known. We sought to investigate the role of angioplasty in patients with anaemia presenting with ACS, and how it affected mortality.
\end{abstract}

Method: Anonymous information on patients with ACS, attending a large multi-ethnic general hospital in Manchester, United Kingdom in the period 2000-2013 was obtained from the local health authority computerised hospital activity analysis register using ICD-10 and OPCS coding systems according to the ACALM protocol.

Results: Out of 25294 patients, $1551(6.1 \%)$ patients had Anaemia as defined by national standards. Of these, $89(5.7 \%)$ patients underwent angioplasty with mean age 65.0 years and were significantly younger than patients who did not undergo angioplasty. Patients who did not undergo angioplasty had higher prevalence of Ischaemic Stroke(6.2\% vs $1.1 \%)$, Heart Failure(36.7\% vs $11.2 \%$ ) and Chronic Kidney Disease(18.7\% vs $4.5 \%)$, whilst angioplasty patients had higher prevalence of previous ACS (27.2\% vs $36.0 \%)$ and peripheral vascular disease(6.8\% vs $7.9 \%$ ). Multi-nominal logistic regression analysis revealed Heart Failure(RR2.16), Ischaemic Stroke(RR3.67) and Chronic Kidney Disease(RR1.39) as significant predictors of mortality in this patient group. Angioplasty(RR7.81) and previous ACS(RR1.88) conferred mortality improvement in this patient group. 


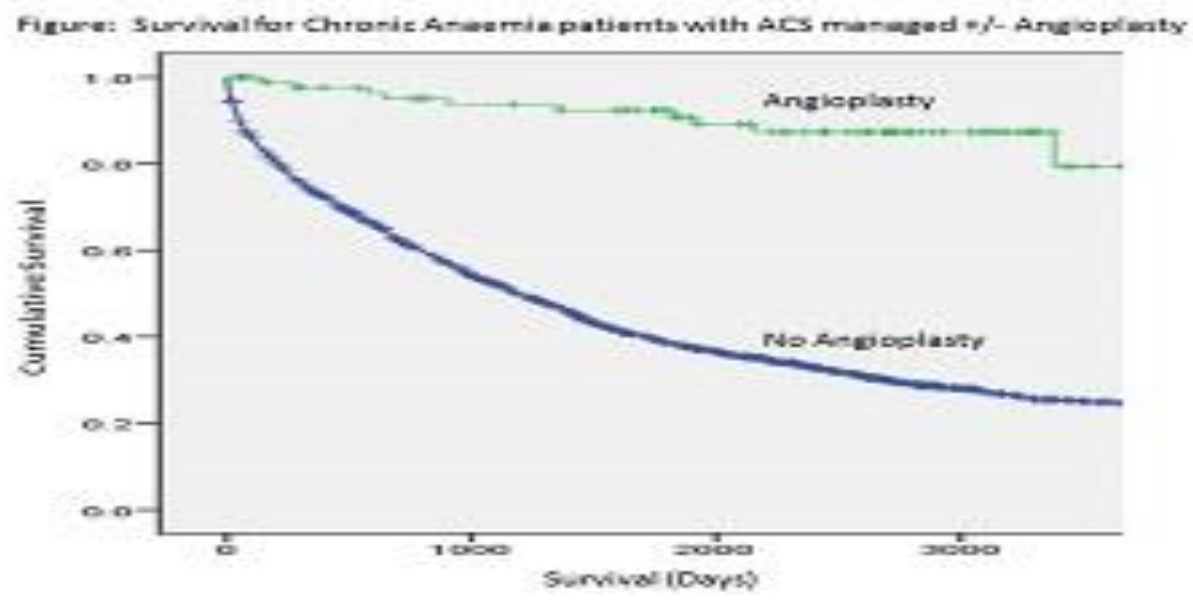

Conclusion: We have shown that angioplasty confers significantly improved mortality in the management of ACS in patients with chronic anaemia. The findings of this study highlight the need for clinicians to conscientiously think about the individual benefits and risks of angioplasty for every patient with chronic anaemia rather than confining to stereotypes.

No conflict of interest 
359

\title{
INTERVENTIONAL CARDIOLOGY AND STENTING
}

\section{ACUTE MYOCARDIAL INFARCTION AND PREGNANCY-CASE IN YOUNG PATIENTS WITH THIRTY-THREE YEARS AND EIGHT MONTHS OF PREGNANCY}

M. De Oliveira

Rio De Janeiro, Brazil

\begin{abstract}
Aims: Acute myocardial infarction is the leading cause of death in the developed world. Risk factors such as diabetes, dyslipidemia, hypertension, smoking, physical inactivity, obesity diet increases the incidence of coronary disease. Nonetheless concomitance of pregnancy, female and youth are not correlated to Ml's incidence and are rare $(0.01 \%)$.
\end{abstract}

Method: We treated a patient of 33 years arrived in the emergency room with infero-dorsal AMI Killip III-IV and eight months pregnant. she was admitted to ICU with acute respiratory failure and cardiogenic shock (BP $=60 \times 40 \mathrm{~mm} \mathrm{Hg}$ ), tachycardia, tachydyspneic, $\mathrm{CB}=120 \mathrm{bpm}$, torpor,,cold extremities, hemodynamically unstable. She was treated with pressor amines (dobutamine $6 \mathrm{mcg} / \mathrm{kg} / \mathrm{min})$, noradrenaline $(6 \mathrm{mcg} / \mathrm{kg} / \mathrm{min})$, volume $(1500 \mathrm{ml} \mathrm{SF}$

$0.9 \%$ ), oxygen $(6 \mathrm{~L} / \mathrm{min})$. Due to worsening of the clinical state we did oro-tracheal intubation and mechanical ventilation and immediate caesarean. There born male fetus in good condition.

Results: After cesarean she was underwent coronary angiography, left ventriculography by right femoral artery access which showed occlusion of the circumflex artery in proximal third.RCA and LAD were free of obstructive lesions. Left ventriculography showed moderate lower hypokinesia and she received (clopidogrel $600 \mathrm{mg} / 200 \mathrm{mg} \mathrm{AAS} / 10.000 \mathrm{IU}$ IV heparin). The LCA was catheterized with catheter guide JL3.5-6F and crossed the lesion with guidewire 0014 mediumsupport. We used vacuum thrombi PRONTO V3 and implemented intensive aspirations of intracoronary thrombus until the establishment of TIMI 3, Then a 3.0x24mm Endeavor stent was placed in the lesion being expanded to 16 atm with excellent angiographic result .

Conclusion: The clinical case shows rare case $(0.01 \%)$ of $\mathrm{AMI}$ in young pregnant women(33 years-8 months)in cardiogenic shock, which was treated with immediate cesarean, thrombus aspiration and implantation coronary stent, Mother and child was discharged 8 days after admission.

No conflict of interest 
360

\title{
INTERVENTIONAL CARDIOLOGY AND STENTING
}

\section{PRE-EXPOSURE TO NORMOBARIC HYPEROXIA HAS NO EFFECT ON MYOCARDIAL INJURY BIOMARKERS AFTER PERCUTANEOUS TRANSLUMINAL CORONARY ANGIOPLASTY}

\author{
A. Mohamadi, M. Namdari, B. Rasoulian, A. Raoufi, A. Nazari \\ Lorestan University of Medical Siences, Madani Hospital, Khoramabad, Iran
}

Aims: It has been determined in animal models that hyperoxia-induced preconditioning could reduce ischemia-reperfusion injury of the heart tissue. Short-term ischemia and subsequent reperfusion occurs unavoidably in coronary angioplasty. The purpose of the present study was to determine the possible effect of oxygen pretreatment in inducing preconditioning during percutaneous transluminal coronary angioplasty (PTCA).

Method: Thirty two patients who had been referred for elective angioplasty were randomly divided into the "control" and the "oxygen" groups. The subjects in the oxygen group were exposed to normobaric oxygen (nearly 70\% O2)via non-rebreathing masks for 1 hour at 12 and 2 hours before PTCA. One hour after the last oxygen pre-exposure period, the patients underwent PTCA (20seconds of balloon inflation, 2 min of reperfusion). The chest pain score and cardiac injury biomarkers(troponin I and CKMB) were assessed 12 hours after the coronary angioplasty.Biomarkers data and chest pain scores were analyzed by Mann-Whitney and Wilcoxon t-test.

Results: Troponin I and CKMB levels were elevated in both groups after angioplasty, but there was not any significant difference between groups in this regard ( $p=0.23$ and 0.47 respectively). The average pain score during the balloon inflation in the oxygen group was lower than in the control group (2.8 \pm 1.2 versus $4.11 \pm 1.21, \mathrm{p}=0.008)$.

Conclusion: Two times of $1 \mathrm{~h}$ pre-exposure to normobaric hyperoxia (nearly $70 \%$ ) at 12 and 2 hours before percutaneous transluminal coronary angioplasty has no significant effect on myocardial injury biomarkers, Troponin I and CKMB.

No conflict of interest 
361

INTERVENTIONAL CARDIOLOGY AND STENTING

\title{
ACUTE ANGIOGRAPHIC, ELECTROCARDIOGRAPHIC AND CLINICAL FINDINGS IN OUT- OF-HOSPITAL CARDIAC ARREST PATIENTS ADMITTED TO URGENT CORONARY INTERVENTION - TEN YEAR EXPERIENCE FROM LARGE INVASIVE CARDIOLOGY CENTER
}

\author{
A. Zelias, A. Trabka-Zawicki, K. Zmudka \\ Institute of Cardiology Jagiellonian University Medical College, Interventional Cardiology Clinic, \\ Krakow, Poland
}

\begin{abstract}
Aims: The main purpose of the study was to evaluate coronary angiography findings in out-ofhospital cardiac arrest $(\mathrm{OHCA})$ patients

Method: We analysed angiographic, electrocardiographic, clinical and laboratory findings of all consecutive OHCA patients admitted to urgent coronary angiography and angioplasty (UCA/PCl) in our institution since 2000 to 2011
\end{abstract}

Results: We analysed 405 patients after OHCA. On coronary angiography acute coronary lesions were found in $82 \%$ of cases. Acute occlusion was present in $48 \%$ of patients, in $34 \%$ there were other acute lesions, i.e. $>90 \%$ stenosis in major coronary artery, lesions with unstable morphology, fresh thrombus or with TIMI $<3$ flow. Among patients diagnosed of ACS, $75 \%$ had ST-segment elevation and 25\% ST-segment depression, inversion of T-wave or non-specific ST$\mathrm{T}$ changes. On univariate analysis the presence of chest pain before cardiac arrest, increasing age, previous myocardial infarction, ST-segment elevation on ECG and the level of hsTpT on admission correlated with finding of significant $(>50 \%)$ coronary artery stenosis. Although in multivariate analysis only ST-segment elevation and/or chest pain before cardiac arrest were independently correlated with acute coronary occlusion or critical stenosis, more than half of nonST elevation OHCA patients had acute coronary lesions. The type of ST segment changes did not have any influence on survival.

Conclusion: Most of OHCA patients admitted to UCA/PCI had acute coronary lesions. Although ST-elevation were found to independently correlate with presence of acute lesion, most patient without ST-segment elevation were also diagnosed of ACS. ECG did not adequately discriminate which OHCA patient should be admitted to UCA/PCI nor affect prognosis.

No conflict of interest 
362

\title{
DRUG THERAPY, CLINICAL TRIALS
}

\section{PHYSIOLOGICAL AND PHARMACHOLOGICAL STUDIES OF ATP SENSITIVE POTASIUM CHANNELS(KATP)IN LANGENDORFF RAT HEART SYSTEM.THE IMPACT OF ISCHEMIA- REPERFUSION}

\author{
F. Revnic ${ }^{1}$, C.R. Revnic ${ }^{2}$, S. Voinea ${ }^{3}$, C. Pena ${ }^{4}$, B. Paltineanu ${ }^{5}$ \\ ${ }^{1}$ NIGG"Ana Aslan", Biology of Aging, Bucharest, Romania \\ 2UMF"Carol Davila, Cardiology, Bucharest, Romania \\ "UMF"Carol Davila", Oncology, Bucharest, Romania \\ "NIGG"Ana Aslan", Biology Aging, Bucharest, Romania \\ ${ }^{5}$ UMF Tg.Mures, General Surgery, Bucharest, Romania
}

\begin{abstract}
Aims: The ATP sensitive $\mathrm{K}$ channels (KATP) have been implicated in myocardium recovery following an ischemic aggression. It has been suggested that phosphorilation of $\mathrm{K}_{\mathrm{ATP}}$, following activation of protein kinase $\mathrm{C}$ via diacylglycerol, leads to the closing of $\mathrm{K}$ channels. The aim of study was concerned with conjugate investigation of these two cellular components in Langendorff retrograde perfused isolated rat heart system
\end{abstract}

Method: After $20^{`}$ stabilisation of heart parameters was induced 45 min ischemia followed by 60 min reperfusion .

Results: After 30 min reperfusion there was a significant reduction in LVPD the decrease in contractility of heart muscle by blocking $\mathrm{K}$ channel with glibenclamid. In the case of association of glibenclamid with DOG, activator of protein kinase $\mathrm{C}$, the reduction of contractile force is less. Activation of protein kinase $\mathrm{C}$ abolishes the blocking effect of $\mathrm{K}$ channels by glibenclamid, in such a way that at the begining of reperfusion cardiac frequency increases above control values, leading to a slight decrease in time Ischaemia/reperfusion modifies functional parameters in isolated heart, and the action of factors which act upon $\mathrm{K}$ channels(either bloking or debloking them) is expressed by modification of these functional parametersThe traditional mechanism of pharmacological agents action to limit lesions produced by experimental ischaemia/reperfusion is related with favorable modification in oxygen supplay balance.

Conclusion: $\mathrm{K}$ channels openers seems to posses an unique antiischaemic mechanism which cannot be explained by improving the oxygen supply or reduction of its consumption, and the antiischaemic effect is produced by a direct cardioprotective action

No conflict of interest 
363

\title{
DRUG THERAPY, CLINICAL TRIALS
}

\section{EPIGALLOCATECHIN-3-GALLATE DECREASED PLATELET AGGREGATION INDEPENDENT OF CONCOMITANT ASPIRIN, CLOPIDOGREL OR TICAGRELOR TREATMENT}

\author{
S.J. Hong, H.J. Joo, D.S. Lim \\ Korea University Anam Hospital, Cardiology, Seoul, Korea
}

\begin{abstract}
Aims: Although epigallocatechin-3e-gallate (EGCG), which is found in high contents in the dried leaves of green tea, has been reported to have anti-platelet effect, synergistic effect of EGCG in addition to under current antiplatelet medications remains to be elucidated.
\end{abstract}

Method: Blood samples were obtained from total 40 participants individuals who took aspirin (ASA, $n=10$ ), clopidogrel (CPD, $n=10$ ), ticagrelor (TCG, $n=10$ ) and no antiplatelet medications (Control, $n=10)$. Ex vivo platelet aggregation and adhesion under various stimulators were analyzed by multiple electrode aggregometry (MEA) and Impact-R. PAC-1 and P-selectin expressions in human platelets were analyzed by flow cytometry.

Results: In MEA analysis (Figure 1), ADP and TRAP-inducible platelet aggregations werewas lower in the CPD and the TCG groups; arachidonic acid (AA)-inducible platelet aggregation was lowered in the ASA group; whereas, collagen (Col)-inducible platelet aggregations were comparable among 4 groups. EGCG reduced ADP, TRAP, AA and Col-inducible platelet aggregation inwith dose-dependent manner in the Control group. There were no significant differences inof ADP, TRAP, AA and Col-inducible platelet aggregation among 4 groups under the maximal EGCG treatment (200umol/L). Impact-R showed no significant differences inof the platelet adhesion (surface coverage) among 4 groups. EGCG had no significant effect on PAC-1 and P-selectin expressions among 4 groups.

Conclusion: Ex vivo treatment of EGCG yielded the maximal suppression of ADP, TRAP, AAASPI and Col-inducible platelet aggregation independent ofto aspirin, clopidogrel or ticagrelor treatment. EGCG had no significant effect on the platelet adhesion and PAC-1 and P-selectin expression. EGCG may be a potential target for the new antiplatelet drug development in the future.

No conflict of interest 
364

\title{
DRUG THERAPY, CLINICAL TRIALS
}

\section{PHARMACOKINETICS (PK), PHARMACODYNAMICS (PD), AND SAFETY OF TICAGRELOR IN CHINESE PATIENTS WITH STABLE CORONARY HEART DISEASE (CHD)}

\author{
H. Li ${ }^{1}$, J. Guo ${ }^{2}$, G.F. Carlson 3 , R. Teng ${ }^{4}$ \\ ${ }^{1}$ Peking University Third Hospital, Clinical Pharmacology Unit, Haiden District- Beijing, China \\ ${ }^{2}$ Peking University Third Hospital, Clinical Pharmacology Unit, Beijing, China \\ ${ }^{3}$ AstraZeneca Pharmaceuticals, Clinical Research, Gaithersburg, USA \\ ${ }^{4}$ AstraZeneca LP, Clinical Pharmacology, Gaithersburg, USA
}

Aims: Ticagrelor and AR-C124910XX (active metabolite) bioavailability are $\sim 40 \%$ higher in Asians versus Caucasians. We investigated the PK, PD and safety of ticagrelor in Chinese CHD patients.

Method: Study: open-label, single-centre, randomised, phase IV (NCT02064985). Patients $(\mathrm{n}=12 /$ dose) on chronic low-dose aspirin (75-100mg/day) received ticagrelor 45,60 or $90 \mathrm{mg}$ (single dose: Days 1 and 7; twice daily [bid]: Days 3-6). Endpoints: PK parameters, inhibition of ADP-induced platelet aggregation (IPA; final-extent), $\mathrm{P}_{2} \mathrm{Y}_{12}$ reaction units (PRU), adverse events (AEs).

Results: All 36 patients completed the study. Dose-proportional (45mg vs $90 \mathrm{mg}$ ) increases in ticagrelor and AR-C124910XX Cmax and AUC0-12h occurred. Day 1: mean IPA was similar up to $2 \mathrm{~h}$, then higher up to $48 \mathrm{~h}$ (all timepoints) with ticagrelor $90 \mathrm{mg}$ versus lower doses. Mean PRU was markedly reduced at $1 \mathrm{~h}$ versus baseline with all doses, with maximum mean PRU reduction $(72-88 \%$ ) at $\sim 3 \mathrm{~h}$. With 45,60 , and $90 \mathrm{mg}$ bid, IPA (Day 7 ) was $>85 \%,>90 \%$ and $>95 \%$,

respectively. PRU values were lower with $90 \mathrm{mg}$ (range 17-32) versus $45 \mathrm{mg}(48-98)$ and $60 \mathrm{mg}$ (36-81). PK, IPA, and PRU differences between $45 \mathrm{mg}$ and $60 \mathrm{mg}$ dosing were small. Proportion of patients with $>1 \mathrm{AE}$ was $33 \%$ (45mg), $33 \%(60 \mathrm{mg})$, and $42 \%(90 \mathrm{mg})$. Most AEs were mild.

Conclusion: Following single and multiple dosing, exposure to ticagrelor and AR-C124910XX increased between 45 and $90 \mathrm{mg}$ doses in Chinese CHD patients. Ticagrelor (45, 60 and 90mg) markedly reduced platelet aggregation, and IPA and PRU magnitude generally increased with increasing doses. Exposure and PD differences between 45 and 60mg were small.

Conflict of Interest Disclosure Sttatement:

For ICCAD, Astrazenca will provide funds for my registration/attendance and travel to the congress to present these data. I have no other conflicts of interest' 
365

\title{
DRUG THERAPY, CLINICAL TRIALS
}

\section{EFFECTS OF A NOVEL HYPOLIPIDEMIC COMPOUND (D-47) ON SERUM LIPID LEVELS OF WHHLMI RABBITS}

 \\ M. Shiomi ${ }^{2}$ \\ ${ }^{1}$ Kobe University Graduate School of Medicine, Division of Comparative Pathophysiology, Kobe, \\ Japan \\ ${ }^{2}$ Kobe University Graduate School of Medicine, Institute for Experimental Animals, Kobe, Japan \\ ${ }^{3}$ Tokushima Bunri University, Medical Chemistry- Faculty of Pharmaceutical Science, Tokushima, \\ Japan
}

Aims: Although effects of statin on serum lipid levels and cardiovascular events are potent, statins' effect on cardiovascular event is limited. Therefore, a compound having different mechanism may be contribute to further reduction of cardiovascular events. We developed a new compound showing hypolipidemic effects.

Method: The compounds were administered to WHHLMI rabbits, an animal model for hypercholesterolemia and coronary atherosclerosis, for 5 weeks at a dose of $30 \mathrm{mg} / \mathrm{kg}$ of D-47, $0.5 \mathrm{mg} / \mathrm{kg}$ of pitavastatin, $50 \mathrm{mg} / \mathrm{kg}$ of bezafibrate, and $300 \mathrm{mg} / \mathrm{kg}$ of EPA. Serum lipid levels were assayed every week by enzymatic methods. Lipoprotein was fractionated with a ultracentrifuge. Expression of mRNA of liver was analyzed with RT-PCR.

Results: Compared to the initiation of drug administration, serum cholesterol levels at the 5 weeks of administration were decreased by $23 \%$ in D-47 group, $28 \%$ in EPA group, and $30 \%$ in pitavastatin group. Serum triglyceride levels were decreased by $41 \%$ in D-47 group, and $34 \%$ in EPA group. However, bezafibrate did not affect serum lipid levels. These decrease in D-47 group was mainly due to a decrease in VLDL fraction. In RT-PCR analyses, expression of FAS was decreased in D-47 group.

Conclusion: D-47 is a potent hypolipidemic compound.

No conflict of interest 


\title{
366 \\ DRUG THERAPY, CLINICAL TRIALS
}

\section{XUEFU ZHUYU PRESCRIPTION FOR THE TREATMENT OF ANGINA PECTORIS: A META- ANALYSIS OF RANDOMIZED CONTROLLED TRIALS}

\author{
S.M. Gao, S. Gao, L. Li, Y.H. Bian, C.Q. Yu \\ Tianjin University of Traditional Chinese Medicine, Tianjin, China
}

The main objective of this study is to quantitatively analyze Xuefu Zhuyu prescription treatment efficacy and safety of coronary heart disease with angina pectoris. In China, Xuefu Zhuyu prescription as a kind of traditional Chinese medicine (TCM) has been widely used in the treatment of angina pectoris, therefore, systematically and objectively evaluate the efficacy of Xuefu Zhuyu prescription is necessary. Retrieving total 8 databases, Cochrane Library, Pubmed, Web of Science, Elsevier, CNKI, Wanfang, CBM, and VIP, the literatures as of April 2015 has been published in both English and Chinese, are absorbed into angina pectoris clinical randomized controlled trials that the treatment of Xuefu Zhuyu prescription alone or merged with conventional Western medicine, and compared with the treatment of conventional Western medicine. All reports which were selected in accordance with strict inclusion criteria will be analyzed with Revman 5.3 software for statistical analysis, end effect index to relative risk (RR) and $95 \%$ confidence interval $(\mathrm{Cl})$. A total of 10 articles were 735 cases of patients with angina pectoris included in the standard, the results showed that the treatment group effect better than the control group $(\mathrm{RR}=1.27 ; 95 \% \mathrm{Cl}=[1.19,1.37])$. No adverse reactions of Xuefu Zhuyu prescription has been reported. Application in the treatment of angina pectoris, Xuefu Zhuyu prescription could improve the efficiency, and has the characteristics of less adverse reaction. However, due to effect of the low quality of the absorbed studies of samples, publication bias and other factors, there has to be more high-quality, multiple-center, large-sample randomized controlled trials to validate the curative effect of Xuefu Zhuyu prescription.

Key words: Xuefu Zhuyu prescription; coronary heart disease; angina pectoris; meta-analysis; randomized controlled trial

Acknowledgments This project was supported by the National Basic Research Program of China (973 Program, 2014CB542902, http://program.most.gov.cn/). The funders had no role in study design and data collection and analysis, decision to publish, or preparation of the paper.

No conflict of interest 
367

\title{
ENDOTHELIAL FUNCTION, VASCULAR BIOLOGY
}

\section{EFFECTS OF CERIUM OXIDE NANOPARTICLES IN HUMAN ENDOTHELIAL CELLS: VIABILITY, APOPTOSIS, INFLAMMATION AND OXIDATIVE STRESS}

\author{
S. Del Turco ${ }^{1}$, G. Ciofani', V. Cappello ${ }^{3}$, T. Navarra ${ }^{1}$, C. Caselli', M. Gemmi ${ }^{3}$, V. Mattoli ${ }^{2}$, \\ G. Basta' \\ ${ }^{1}$ Institute of Clinical Physiology, Council of National Research, Pisa, Italy \\ ${ }^{2}$ Center of MicroBioRobotics@SSSA, Istituto Italiano di Tecnologia- Pontedera Pisa- Italy, Pisa, \\ Italy \\ ${ }^{3}$ Center for Nanotechnology Innovation @NEST, Istituto Italiano di Tecnologia- Pisa- Italy, Pisa, \\ Italy
}

Aims: The endothelium plays a key role in cardiovascular physiopathology and represents an important target for drug or gene therapy. Cerium oxide nanoparticles (nanoceria) have attracted much attention due to their antioxidant potential. We explored the cytocompatibility and the antiinflammatory, antioxidant and anti-apoptotic effects of nanoceria on endothelial umbilical vein endothelail cells (HUVECs).

Method: HUVECs were incubated for $24 \mathrm{~h}$ and $48 \mathrm{~h}$ with nanoceria in cell culture medium at 50 $\mu \mathrm{g} / \mathrm{mL}$. Cell viability and proliferation was assayed by WST-1 assay. Flow cytometry analysis was used to evaluate apoptosis and microparticle release. Vascular adhesion molecule (VCAM-1) expression by surface enzyme immunoassay, intracellular ROS production by the DCF assay, IL6 release by ELISA assay and nanoparticle uptake evaluated by transmission electron microscopy (TEM).

Results: Nanoceria did not affect cell vitality and did not induce apoptosis in HUVECs at any of the incubation time. Nanoceria reduced the $\mathrm{H} 2 \mathrm{O} 2(25 \mu \mathrm{mol} / \mathrm{L})$-induced ROS production in a timedependent manner $(-40 \% \pm 10 \%,-26 \% \pm 8 \%, \mathrm{p}<0.05$, at 48 and $24 \mathrm{~h}$, respectively) and VCAM-1 exposure induced by TNF- $\alpha(25 \mathrm{ng} / \mathrm{mL})(-35 \% \pm 6 \%$ at $24 \mathrm{~h}, \mathrm{p}<0.01)$. Moreover, nanoceria significantly reduced IL- 6 release in cell culture medium after stimulation with TNF- $\alpha$ (38 $\pm 1.9 \mathrm{pg} / \mathrm{mL}$ vs TNF- $\alpha 45 \pm 2.25 \mathrm{pg} / \mathrm{mL}$, at 24 and $48 \mathrm{~h}$ respectively, $\mathrm{p}<0.05)$. TEM analysis revealed that nanoceria was uptake by HUVECs and after $24 \mathrm{~h}$ its localization found mostly in the cytoplasm. We have also demonstrated that HUVECs released microparticles loaded with nanoceria when stressed by serum deprivation.

Conclusion: These results indicate that nanoceria do not affect endothelial cell biology and may be helpful for more rational application in biomedical fields

No conflict of interest 
368

\title{
ENDOTHELIAL FUNCTION, VASCULAR BIOLOGY
}

\section{THE MELANOCORTIN RECEPTOR-1 POSITIVELY REGULATES HUMAN ARTERY ENDOTHELIAL CELL MIGRATION}

\author{
F. Saporiti, L. Piacentini, E. Bono, M. Chiesa, V. Alfieri, G.I. Colombo \\ Centro Cardiologico Monzino, Unit of Immunology and Functional Genomics, Milan, Italy
}

Aims: Endothelial cell (EC) injury is central to the pathogenesis of atherosclerosis, thrombosis or vascular surgery complications. Targeting melanocortin receptor-1 (MC1R), which belongs to a hormonal signaling pathway exerting multiple homeostatic and protective actions, has been shown to attenuate endothelial dysfunction in a pre-established atherosclerosis mouse model. We sought to investigate whether human macrovascular ECs express any MCRs and what their action is.

Method: Detection of melanocortin system components in primary human aortic and coronary ECs was performed by real-time PCR, Western-Blot, and immunocytochemistry. Intracellular cAMP and $\mathrm{Ca}^{2+}$ concentrations were measured by enzyme immunoassay and the Fluo-4 fluorescent probe, respectively. Directional cell migration assay was used for in vitro wound healing experiments. Gene expression profiling was performed with Illumina HumanHT-12_v4 BeadChips.

Results: Confluent human macrovascular ECs expressed MC1R, whose stimulation raised intracellular CAMP, but no other MCR nor proopiomelanocortin, the common precursor of MCR ligands. The high-affinity MC1R ligand, the alpha-melanocyte stimulating hormone, was able to accelerate EC migration by enhancing the $\mathrm{Ca} 2+$ signaling cascade. This was reversed by an MC1R antagonist. Microarray analysis allowed identifying time-dependent dynamic modulation of genes involved in ECM-receptor interaction, cell adhesion, and vessel development. The coregulation of these cellular processes appeared to drive the faster closure of the wound.

Conclusion: These results provide evidence of a novel function of peripherally expressed MC1R, whose activation may participate in preventing and healing endothelial dysfunction in macrovascular arteries. EC MC1R could represent a target for therapeutic strategies aimed at repairing endothelial injury and denudation in a variety of vascular pathological conditions.

No conflict of interest 
369

\title{
ENDOTHELIAL FUNCTION, VASCULAR BIOLOGY
}

\section{EFFECTS OF ALPHA-MELANOCYTE-STIMULATING HORMONE ON VASCULAR SMOOTH MUSCLE CELL PHENOTYPIC SWITCHING, MIGRATION AND PROLIFERATION}

\author{
F. Saporiti, V. Alfieri, L. Piacentini, E. Bono, M. Chiesa, G.I. Colombo \\ Centro Cardiologico Monzino, Unit of Immunology and Functional Genomics, Milan, Italy
}

\begin{abstract}
Aims: It has been shown that the melanocortin peptide alpha-melanocyte-stimulating hormone $(\alpha-M S H)$, the most potent endogenous anti-inflammatory factor, was able to attenuate vascular dysfunction and plaque inflammation in a pre-established atherosclerosis mouse model. We investigated whether human vascular smooth muscle cells (VSMCs) express a functional melanocortin system and whether a-MSH could affect VSMC phenotypic switching, migration and proliferation, the hallmarks of vascular remodeling.
\end{abstract}

Method: Detection of melanocortin system components in primary human aortic and coronary SMCs was performed by real-time PCR, Western-Blot, and immunocytochemistry. VSMC differentiation and migration was analyzed using wound-healing and transwell assays and timelapse imaging by confocal microscopy. VSMC proliferation was evaluated by BrdU colorimetric assay. Phenotypic markers were detected by immunofluorescence.

Results: We showed that macrovascular SMCs express two MCR types, MC1R and MC4R, but not the $\alpha-\mathrm{MSH}$ precursor proopiomelanocortin. In vitro stimulation with $\alpha-\mathrm{MSH}$ promoted VSMC differentiation from a synthetic, proliferative, and migratory phenotype with an epithelioid morphology, to a contractile, quiescent phenotype with an elongated, spindle-shaped conformation. $\alpha-\mathrm{MSH}$ enhanced the expression of smooth muscle $\alpha$-actin, which is a marker of the contractile phenotype, while reducing vimentin and thrombospondin, markers of the synthetic phenotype. MCR activation by $\alpha-M S H$ inhibited VSMC proliferation and slowed down migration both in wound-healing assay, with a peak effect at 9-12 h, and in transwell migration assay, using PDGF-BB as chemoattractant.

Conclusion: These results unveil a novel key function for peripherally expressed MCRs. VSMC MCRs may have a role in vascular pathophysiology, thus suggesting a possible therapeutic use of melanocortins in preventing pathologic vascular remodeling.

No conflict of interest 
370

\title{
ENDOTHELIAL FUNCTION, VASCULAR BIOLOGY
}

\section{THE ACCUMULATED HYPOXEMIA BY SLEEP DISORDERED BREATHING AFFECTS VASCULAR ENDOTHELIAL FUNCTION ESPECIALLY IN THE PATIENTS WITH ISCHEMIC CARDIAC FAILURE}

\author{
H. Sawatari' , S.I. Ando², M. Nishizaka', M. Miyazono ${ }^{3}$, S. Inoue ${ }^{4}$, T. Sakamoto ${ }^{4}$, T. Tokunou ${ }^{4}$, \\ H. Chishaki ${ }^{5}$, A. Chishaki ${ }^{1}$ \\ ${ }^{1}$ Kyushu University Graduate School of Medical Sciences, Department of Health Sciences, \\ Fukouka, Japan \\ ${ }^{2}$ Kyushu University Hospital, Sleep Apnea Center, Fukuoka, Japan \\ ${ }^{3}$ Fukuoka Prefectural University, School of Nursing, Fukuoka, Japan \\ ${ }^{4}$ Kyushu University Graduate School of Medical Sciences, \\ Department of Cardiovascular Medicine, Fukouka, Japan \\ ${ }^{5}$ Takayama Hospital, Department of Internal Medicine, Fukuoka, Japan
}

\begin{abstract}
Aims: Impairment of vascular endothelial function (VEF) is one of the major causes of coronary artery disease (CAD). It is well known that sleep-disordered breathing (SDB) impairs VEF. However, it has been unclear what SDB-related indices most properly predict VEF in patients with heart failure (HF), and thus we assessed those relationships in this study.
\end{abstract}

Method: We evaluated SDB and VEF in 24 patients with HF (Male: 18, Age: $58 \pm 14$ y.o.) from variable causes (7: Ischemic, 10: Idiopathic cardiomyopathy, and 7: Others). They underwent polysomnography and flow-mediated vasodilation (FMD) measurement. We assessed the relationships between FMD and SDB-related indices, including traditional indices (i.e., apneahypopnea index $[\mathrm{AHI}]$ and lowest arterial saturation level [Lowest SpO2]) and time desaturation summation index (TDS) that we had newly developed to assess the accumulated hypoxemia by SDB (Formula: TDS=Total sleep time $x$ mean SpO2 during sleep).

Results: Their mean left-ventricular ejection fraction was $27.4 \pm 10.0 \%$. They had impaired VEF (FMD: $5.3 \pm 2.7 \%$ ) with wide range of SDB (AHI: $8.8 \pm 13.8 /$ hour, Lowest SpO2: $85.1 \pm 6.6 \%$, TDS: $49.5 \pm 19.2 \% *$ hour). FMD significantly correlated to age, diastolic blood pressure, hypertension and TDS $(p<0.05)$ but not traditional indices of SDB. Furthermore, the correlation in TDS was higher in the patients with ischemia than those without ( $r=-0.69$ vs. $r=-0.36)$. The significance in age and TDS did not change even after the adjustment of confounding factors $(p<0.05$; both).

Conclusion: The accumulated hypoxemia in SDB, represented by TDS, closely related with VEF in patients with HF, especially in the patients with ischemia. Treatment to hypoxemia in SDB might contribute to prevention of CAD.

No conflict of interest 


\begin{tabular}{|c|c|}
\hline Author Name & Catalog No. \\
\hline Abbaszadeh, M. & 182 \\
\hline Abdalla, D.S.P. & 245 \\
\hline Abdel-Sayed, S. & 210 \\
\hline Abdellatif, M. & 223 \\
\hline Abraham, V. & 303 \\
\hline Abreu-Gonzalez, P. & 274 \\
\hline Abreu, P. & 296 \\
\hline Açar, B. & 252 \\
\hline Adachi, $\mathrm{H}$. & 371 \\
\hline Adams, S. & 88 \\
\hline Adascalitei, L.C. & 354 \\
\hline Adone, G. & 72 \\
\hline Adrio Nazar, B. & $325,337,338$ \\
\hline Ae-Young, H. & 352 \\
\hline Aiuchi, T. & 162 \\
\hline Al Kayal, T. & 288 \\
\hline Alaeddini, F. & 201 \\
\hline Alavinia, S.E. & 201 \\
\hline Alberto, G.L. & 329 \\
\hline Albustan, S. & 31 \\
\hline Aldohmy, S.I. & 168 \\
\hline Alegria-Barrero, E. & $167,294,308,309$ \\
\hline Alfieri, V. & 368,369 \\
\hline Alonso-Viteri, S. & 294, 308, 309 \\
\hline Alpert, J.S. & 19 \\
\hline Alrashdan, I. & 31 \\
\hline Alserri, A. & 31 \\
\hline Alshosha, A. & 65 \\
\hline Amemiya, $\mathrm{T}$. & 351 \\
\hline Ami, K. & 348 \\
\hline Amirzadegan, A. & 73,255 \\
\hline Ando, S.I. & 370 \\
\hline Andraloits, I. & 202 \\
\hline Andrsova, I. & 183 \\
\hline Angoulvant, D. & 235 \\
\hline Anson, $\mathrm{T}$. & 180 \\
\hline Antoniou, A. & 349 \\
\hline Ao, L. & 263 \\
\hline Apanova, O. & $283,284,347$ \\
\hline Aprami, T. & 203 \\
\hline Arakaki, R. & 324,326 \\
\hline Arencibia, A. & 245 \\
\hline Argyropoulou, E. & 349 \\
\hline
\end{tabular}


Aronheim, A.

Arun, $\mathrm{K}$.

Asanin, M.

Aslanabadi, N.

Attwell, $\mathrm{S}$.

Auriti, A.

Avanzas, $\mathrm{P}$.

Averkov, $\mathrm{O}$.

Ayatollahi, A.

Ayele, G.

Azhar, $\mathrm{S}$.

Aziz, M.

Bacakova, L.

Badiu, D.C.

Bagsit, N.L.

Bai, L.

Balbay, Y.

Balci, M.M.

Balogh, L.

Bangou, J.

Barahona, J.M.

Barancik, M.

Barillà, C.S.

Bartekova, M.

Bash, L.

Bassano, C.

Bassetti, B.

Basta, G.

Baumann, M.

Beaney, K.

Behmanesh, $\mathrm{M}$.

Beissel, J.

Belleyo-Belkasem, C.

Belleyo, C.

Benak, A.

Bendžala, M.

Benoist, L.

Bereczky, z.

Berti, $\mathrm{S}$.

Bertoldo, C.

Besada, E.

Bezon, E.

Bian, Y.

Bian, Y.H.
28

218

$91,129,280,289$

290

160

70

177, 207, 232, 249, 274, 296

283,284

29

86

160

203

244

315

230

185,341

252

252

297

300

294

225, 227

70

225, 227

196

322

163

345, 367

197, 104

300

234, 256, 257

104

296

273, 287

275

312

235

297

$288,345,353$

322

308

209

328

366 
Bilic, I.

Bilkhu, R.

Birnbaum, Y.

Bitran, D.

Bitsis, T.

Blaha, M.

Blázquez, M.A.

Blumenthal, D.

Bobadilla, B.

Bochkov, V.

Bodi, V.

Boehm, M.

Boknik, P.

Bonanad Lozano, C.

Bonanad, C.

Bongartz, L.

Bonilla, V.

Bono, E.

Borer, J.S.

Borges, S.

Boroumand, M.

Borowski, A.

Borrego, L.

Bošnjak, I.

Bostanitis, I.

Botto, N.

Bovio, E.

Brener, S.J.

Burchell, J.

Burlui, A.

Bushkevich, M.

Butnaru, A.

Cackowska, M.

Callejo, F.

Calvo, I.

Cambronero, E.

Camici, J.

Camillo Pavesi, P.

Cao, M.

Capilla, E.

Cappello, V.

Čaprnda, M.

Cardarelli, R.

Cardona Olle, M.
105

212

134

65

254, 349

275

167, 294, 308, 309

298

226, 233

35

90, 270

25

219

270

90

69

$276,329,350$

$84,368,369$

25

30

234, 256, 257

211

167, 309

241

254

$288,345,353$

208, 322

10,86

356

305

202

65

262

207

125

90

147

9

357

271

367

312

32

181 


\begin{tabular}{|c|c|}
\hline Carlson, G.F. & 364 \\
\hline Caruana, E. & $206,327,340$ \\
\hline Casares, M. & 309 \\
\hline Casellai, G. & 9 \\
\hline Caselli, C. & 367 \\
\hline Cavalcante, M.F. & 245 \\
\hline Čavka, A. & 241 \\
\hline Cellarier, G. & 271 \\
\hline Cequier-Fillat, A. & 199 \\
\hline Cerny & 285 \\
\hline Chadet, S. & 235 \\
\hline Chakraborty, K. & 127 \\
\hline Chandran, S. & 87,358 \\
\hline Charuzii, Y. & 146 \\
\hline Chatzianagnostou, $\mathrm{K}$. & 345 \\
\hline Chen, $\mathrm{E}$. & 124 \\
\hline Chen, $\mathrm{Q}$. & 45,56 \\
\hline Cheng, G. & 81 \\
\hline Cheng, $\mathrm{H}$. & 263 \\
\hline Cheng, W. & 64 \\
\hline Chesario, M. & 203 \\
\hline Chiesa, M. & $84,368,369$ \\
\hline Chin, E. & 243 \\
\hline Chin, J. & 102 \\
\hline Chishaki, A. & 370 \\
\hline Chishaki, H. & 370 \\
\hline Chodick, G. & 196 \\
\hline Choi, H.H. & 265 \\
\hline Choi, S.H. & 336 \\
\hline Choi, Y.S. & 348 \\
\hline Choplain, J.N. & 209 \\
\hline Chorro, F.J. & 90,270 \\
\hline Chou, J. & 298 \\
\hline Chrzan, I. & 253 \\
\hline Chul-Soo, P. & 348 \\
\hline Ciofani, G. & 367 \\
\hline Clark, R. & 128 \\
\hline Clavel, L. & $177,232,249$ \\
\hline Clua-espuny, J.L. & 258,277 \\
\hline Cmrečnjak, J. & 105,279 \\
\hline Cohen, R. & 161 \\
\hline Colivicchi, F. & 70 \\
\hline Colombo, G. & 84 \\
\hline Colombo, G.I. & 368,369 \\
\hline
\end{tabular}




\begin{tabular}{|c|c|}
\hline Coman, S. & 305,354 \\
\hline Cooper, J. & 300 \\
\hline Cowan, L. & 344 \\
\hline Creemers, E. & 101 \\
\hline Cuevas, A. & 245 \\
\hline Ćwiklińska, A. & 261,262 \\
\hline Czer, L. & 64 \\
\hline Czuriga, I. & 297 \\
\hline Dąbkowski, K. & 261,262 \\
\hline Dalyanoglu, H. & 211 \\
\hline Danila, M. & 235 \\
\hline Dastras, M. & 293 \\
\hline Dauerman, H.L. & 20 \\
\hline David, C.H. & 209 \\
\hline Dawn, B. & 81 \\
\hline De Caterina, A. & 288 \\
\hline De Guzman, M.B. & 230 \\
\hline De la Rosa, A. & 273,287 \\
\hline De Leon, F.R.G. & 333 \\
\hline De Oliveira, M. & $179,295,359$ \\
\hline De Robertis, M. & 64 \\
\hline de Ronde, M. & 101 \\
\hline de Winther, M. & 101 \\
\hline Deckers, J. & 69 \\
\hline Dedovic, V. & $91,280,281,301$ \\
\hline Dejan, M. & 129 \\
\hline Del Turco, S. & 345,367 \\
\hline Del Valle, R. & $177,232,249$ \\
\hline Delfini, $M$. & 72 \\
\hline Demarest, S. & 104 \\
\hline Dhalla, N.S. & 121 \\
\hline Di Lisa, F. & 152 \\
\hline Di Pasquale, G. & 9 \\
\hline Diarte, J.A. & 125 \\
\hline Diaz, H. & 294 \\
\hline Dimitriadis, D. & 254,349 \\
\hline Dimitriadis, G. & 254,349 \\
\hline Dlouha, D. & 222 \\
\hline Dmitriev, V. & 347 \\
\hline Dobras, J. & $129,280,281,289,301$ \\
\hline Dominguez-Rodriguez, A. & 274,296 \\
\hline Dong-Kie, K. & 335 \\
\hline Doo-II, K. & 335 \\
\hline Dovinova, I. & 227 \\
\hline
\end{tabular}




\begin{tabular}{|c|c|}
\hline Drzewiecki, K. & 219 \\
\hline $\mathrm{Du}, \mathrm{Q}$. & 310 \\
\hline Dukát, A. & 311,312 \\
\hline Duque, $A$. & 273,287 \\
\hline Durán Muñoz, D. & $337,338,325$ \\
\hline Dutra de Souza, H.C. & 240,242 \\
\hline Ejima, E. & 166 \\
\hline Elsawy, M.M. & 168 \\
\hline Elseweidy, M.M. & 168 \\
\hline Escribano, $\mathrm{P}$. & 276 \\
\hline Eskandar, J. & 219 \\
\hline Esther, C. & 270 \\
\hline Eva, G. & 329 \\
\hline Evans, J. & 356 \\
\hline Faridamin, $\mathrm{N}$. & 180 \\
\hline Fernández González, A. & 337,338 \\
\hline Fernández González, Á.L. & 325 \\
\hline Ferrari, R. & 6 \\
\hline Ferreira-Caro, F. & 258,277 \\
\hline Ferrerira, I. & 181 \\
\hline Filippini, E. & 9 \\
\hline Filova, E. & 244 \\
\hline Fink, D. & 65 \\
\hline Fink, G. & 198 \\
\hline Finley, J. & 88 \\
\hline Fischman, D. & 88 \\
\hline Floren, K.G. & 278,319 \\
\hline Fogarassyova, M. & 225,227 \\
\hline Forcadell-Arenas, $\mathrm{T}$. & 258 \\
\hline Ford, L. & 25 \\
\hline Foucan, L. & 300 \\
\hline Francia, R. & 238 \\
\hline Franco Tortorici, G. & 9 \\
\hline Franco, A.I. & 308 \\
\hline Freitas, A.I. & 30,302 \\
\hline Freitas, C. & 302 \\
\hline Freitas, S. & 30 \\
\hline Fuertes Ferre, G. & 125 \\
\hline Fujii, H. & 264 \\
\hline Fujikake, A. & 247,248 \\
\hline Fullerton, D. & 263 \\
\hline Gackowski, A. & 253 \\
\hline Gajdošík, J. & 311,312 \\
\hline Galache, G. & 125 \\
\hline
\end{tabular}




\begin{tabular}{|c|c|}
\hline Galati, A. & 72 \\
\hline Gambini, E. & 163 \\
\hline Gamra, H. & 260 \\
\hline Gao, R. & 146 \\
\hline Gao, S. & 366 \\
\hline Gao, S.M. & 366 \\
\hline Garcia Carro, J. & 337,338 \\
\hline Garcia Lledó, J.A. & 350 \\
\hline García-Blas, S. & 90 \\
\hline Garcia-carro, J. & 325 \\
\hline Garcia-lledo, A. & 276 \\
\hline Garcia, E. & 350 \\
\hline Garcia, R. & 276 \\
\hline Garvan, C. & 344 \\
\hline Gavara, J. & 90,270 \\
\hline Gemmi, M. & 367 \\
\hline George, K. & 303 \\
\hline Gerard, L. & 260 \\
\hline Gergs, U. & 219 \\
\hline Ghaffari, S. & 201, 290, 291 \\
\hline Ghojazadeh, M. & 291 \\
\hline Gil-Guillen, V.F. & 258 \\
\hline Gilliam, J. & 344 \\
\hline Ginghina, C. & 316,330 \\
\hline Girgis, M. & 81 \\
\hline Gliwińska, A. & 261,262 \\
\hline Godehardt, E. & 211 \\
\hline Goldman, D. & 298 \\
\hline Goldstein, J.A. & 13 \\
\hline González-Cristofol, C. & 167 \\
\hline Gonzalez-Gonzalez, J. & 296 \\
\hline Gonzalez-Henares, A. & 277 \\
\hline Gonzalez-Henares, M.A. & 258 \\
\hline Goodarzynejad, H. & $234,256,257$ \\
\hline Gorantla, R.S. & 87,358 \\
\hline Goravanchi, F. & 285 \\
\hline Gorcyca, K. & 102 \\
\hline Gradišer, M. & 105,279 \\
\hline Graidis, C. & 254,349 \\
\hline Grand Bauza, A. & 338 \\
\hline Granda Bauza, A. & 337 \\
\hline Gruchała, M. & 261 \\
\hline Guang-Won, S. & 335 \\
\hline Gukop, P. & 212 \\
\hline
\end{tabular}




\begin{tabular}{|c|c|}
\hline Guo, J. & 364 \\
\hline Gurina, N. & 259 \\
\hline Gutman, N. & 212 \\
\hline Guzman, D. & 245 \\
\hline Gyöngyösi, M. & 118 \\
\hline Hagberg, J. & 243 \\
\hline Haj Ahmed, S. & 260 \\
\hline Halon, D.A. & 143 \\
\hline Hamdi, E. & 107 \\
\hline Hammoudeh, R. & 106 \\
\hline Hamoud, S. & 161 \\
\hline hare, $C$. & 71 \\
\hline Harper, R. & 356 \\
\hline Hartjes, T. & 344 \\
\hline Hasebe, N. & 334 \\
\hline Hashimoto, M. & 351 \\
\hline Hashimoto, S. & 355 \\
\hline Hassan, A. & 107,161 \\
\hline Hassan, $\mathrm{K}$. & 238 \\
\hline Hata, T. & 355 \\
\hline Hatano, Y. & 351 \\
\hline Hayashi, M. & 162 \\
\hline Hayek, T. & 161 \\
\hline $\mathrm{He}, \mathrm{M}$. & 223 \\
\hline Hemker, J. & 127 \\
\hline Hennig, B. & 224 \\
\hline Henriques, E. & 302 \\
\hline Hernandez, J.M. & $177,232,249$ \\
\hline Higashi, A. & 247,248 \\
\hline Hill, J. & 153 \\
\hline Hink, U. & 127 \\
\hline Holicka, M. & 183 \\
\hline Hong, K.S. & 265 \\
\hline Hong, S.J. & $34,236,363$ \\
\hline Hosseinkhah, Y. & 290 \\
\hline Huang, F. & 164 \\
\hline Huang, J. & 164 \\
\hline Huang, S. & 164 \\
\hline Hubacek, J. & 222 \\
\hline Humphries, S.E. & 300 \\
\hline Huo, Y. & 124 \\
\hline Hur, K.Y. & 336 \\
\hline Ikeda, K. & 339 \\
\hline Inafuku, H. & 324 \\
\hline
\end{tabular}




\begin{tabular}{|c|c|}
\hline Inoue, $\mathrm{H}$. & 166 \\
\hline Inoue, S. & 370 \\
\hline Irina, G. & 213 \\
\hline Irzmanski, R. & 317,318 \\
\hline Ishida, I. & 251 \\
\hline Ishii, Y. & 339 \\
\hline Ismael, F. & 31 \\
\hline Itabe, $\mathrm{H}$. & 162 \\
\hline Ito, S. & 351 \\
\hline Ivanes, F. & 235 \\
\hline Izquierdo, M. & 273 \\
\hline Izquierdo, M.M. & 287 \\
\hline Jaafar, N. & 237 \\
\hline Jaffe, A.S. & 89 \\
\hline Jahagirdar, R. & 160 \\
\hline Jalali, A. & $234,256,257$ \\
\hline Jankowski, M. & 261,262 \\
\hline Jebavy, P. & 282 \\
\hline Jeenduang, N. & 165 \\
\hline Jena, $A$. & 298 \\
\hline Jiang, J. & 124 \\
\hline Jillian, K. & 107 \\
\hline $\operatorname{Jin}, X$ & 57 \\
\hline Johansson, J. & 160 \\
\hline Johnson, F. & 164 \\
\hline Joo, H.J. & 363 \\
\hline Jose Vicente, M. & 90 \\
\hline Jose, J.V. & 303 \\
\hline Jovanovic, L. & 129 \\
\hline Juarez-Prera, R. & 274 \\
\hline Jugdutt, B.I. & 371 \\
\hline Jujo, K. & 251 \\
\hline Juretič, A. & 306 \\
\hline Kala, P. & 183,272 \\
\hline Kaljadka, M. & 202 \\
\hline Kanjuh, V. & $91,129,280,281,289,301$ \\
\hline Kanovsky, J. & 183,272 \\
\hline Kaoubaa, N. & 260 \\
\hline Kaplan, M. & 161 \\
\hline Kapusta, J. & 317,318 \\
\hline Kara, M. & 252 \\
\hline Karakostas, G. & 349 \\
\hline Karapanagiotidis, G.T. & 212 \\
\hline Karasavvidis, V. & 254,349 \\
\hline
\end{tabular}




\begin{tabular}{|c|c|}
\hline Karpov, Y. & 259 \\
\hline Kassaian, S.E. & 201 \\
\hline Kato, R. & 162 \\
\hline Katona, É. & 297 \\
\hline Kautzner, J. & 275 \\
\hline Kee, S. & 285 \\
\hline Keltai, M. & 137 \\
\hline Kermani, A. & 29 \\
\hline Khalifa, A.A. & 209 \\
\hline Khan, I. & 102 \\
\hline Khomitskaya, Y. & 283,284 \\
\hline Ki-Hun, K. & 335 \\
\hline Kim, A. & 251 \\
\hline Kim, J.H. & 336 \\
\hline Kim, K.M. & 336 \\
\hline Kim, U. & 231 \\
\hline Kim, Y.H. & 352 \\
\hline Kim, Y.J. & 231 \\
\hline Kirkels, H. & 69 \\
\hline Kise, Y. & 324 \\
\hline Klainman, S. & 198 \\
\hline Kleinbongard, P. & 116 \\
\hline Kobayashi, T. & 339 \\
\hline Koc, L. & 183,272 \\
\hline Kodera, S. & 184 \\
\hline Koichubekov, B. & 313,314 \\
\hline Koike, T. & 365 \\
\hline Koji, H. & 166 \\
\hline Kojouri, J. & 201 \\
\hline Kok, M. & 101 \\
\hline Kolodzey, G. & 297 \\
\hline Komajda, M. & 25 \\
\hline Komatsu, S. & 247,248 \\
\hline Komatsu, T. & 247,248 \\
\hline Komorníková, A. & 312 \\
\hline Konarik, M. & 244 \\
\hline Konno, S. & 299 \\
\hline Koren, L. & 28 \\
\hline Kortas-Stempak, B. & 261,262 \\
\hline Kotaro, N. & 166 \\
\hline Kowalski, A.M. & 285 \\
\hline Kowalski, J. & 317,318 \\
\hline Król, E. & 262 \\
\hline Kruth, $\mathrm{H}$. & 57 \\
\hline
\end{tabular}




\begin{tabular}{|c|c|}
\hline Ku, H.C. & 286 \\
\hline Kuchta, A. & 261,262 \\
\hline Kukreja, R. & 78 \\
\hline Kulikowski, E. & 160 \\
\hline Kumar, N.T. & 103 \\
\hline Kuniyoshi, Y. & 324,326 \\
\hline Kuo, Y.H. & 286 \\
\hline Kurganovich, S. & 213 \\
\hline Kuroyanagi, T. & 247,248 \\
\hline Kursar, M. & 279 \\
\hline Kwon, S.U. & 342,343 \\
\hline Labedz-Maslowska, A. & 81 \\
\hline Lacalzada, J. & 273,287 \\
\hline Laita, S. & 125 \\
\hline Lanas, F. & 226,233 \\
\hline Landers-ramos, R. & 243 \\
\hline Langtree, J. & 71 \\
\hline Lanska, V. & 222 \\
\hline Lansky, A. & 86 \\
\hline Larifla, L. & 300 \\
\hline Lavu, D. & 87 \\
\hline Lavu, N. & 358 \\
\hline Laynez, I. & 273,287 \\
\hline Le Bihan, $\mathrm{E}$. & 197 \\
\hline Lechuga-Duran, I. & 277 \\
\hline Lee, C.H. & 231 \\
\hline Lee, S.Y. & 331 \\
\hline Lefort, C. & 235 \\
\hline Lerios, J.K. & 319 \\
\hline LI, H. & 124,364 \\
\hline Li, L. & 366 \\
\hline $\mathrm{Li}, \mathrm{X}$ & 124 \\
\hline Li, Y. & 124 \\
\hline Liest $\varnothing \mathrm{l}, \mathrm{K}$. & 103 \\
\hline Lim, D.S. & $34,236,363$ \\
\hline Limongelli, G. & 72 \\
\hline Lio, A. & 208 \\
\hline Liu, P. & 185,341 \\
\hline Llosa, J.C. & 207 \\
\hline Løberg, E.M. & 103 \\
\hline Loez-Otero, D. & 232 \\
\hline Loiaconi, V. & 70 \\
\hline López-Lereu, M.P. & 90,270 \\
\hline Lopez-Menendez, J. & 207 \\
\hline
\end{tabular}




\begin{tabular}{|c|c|}
\hline Lopez-Otero, D. & 177,249 \\
\hline Lopez-Pablo, C. & 277 \\
\hline Lorentz, N. & 197,104 \\
\hline Losi, P. & 288 \\
\hline Lozano, T. & 199 \\
\hline Lysjonok, E. & 213 \\
\hline Ma, T. & 124 \\
\hline Maclssac, A. & 356 \\
\hline MacLachlan, $\mathrm{H}$. & 71 \\
\hline Maddaluna, A. & 72 \\
\hline Maden, $\mathrm{O}$. & 252 \\
\hline Mæhlen, J. & 103 \\
\hline Mainra, R. & 107 \\
\hline Malek, I. & 222 \\
\hline Mandzák, Á. & 297 \\
\hline Mani, $\mathrm{H}$. & 126 \\
\hline Mann, M. & 239 \\
\hline Mansel, $\mathrm{H}$. & 107 \\
\hline Marenzi, G. & 84 \\
\hline Margolis, L. & 54 \\
\hline Marí López, B. & 287 \\
\hline Marí, B. & 273 \\
\hline Markova, M. & 244 \\
\hline Marks, E. & 218 \\
\hline Marotta, M. & 353 \\
\hline Martin Yuste, V. & 181 \\
\hline Martín, M. & 273,287 \\
\hline Martin, $P$. & 183 \\
\hline Martinez Cereijo, J.M. & $325,337,338$ \\
\hline Masoudkabir, F. & 201 \\
\hline Matejkova, E. & 244 \\
\hline Matsuyama, A. & 334 \\
\hline Mattoli, V. & 367 \\
\hline Mavrogianni, A. & 254 \\
\hline Mazzone, A. & $288,345,353$ \\
\hline McCarey, M. & 88 \\
\hline Meana, B. & 207 \\
\hline Mehmedbegovic, Z. & $91,280,281,289,301$ \\
\hline Mehran, R. & 86 \\
\hline Meijers, J. & 101 \\
\hline Meilin, E. & 161 \\
\hline Mejer, A. & 317 \\
\hline Méndez, C. & 273,287 \\
\hline Mendonca, M.I. & 30,302 \\
\hline
\end{tabular}




\begin{tabular}{|c|c|}
\hline Meng, $X$. & 263 \\
\hline Merin, $\mathrm{O}$. & 65 \\
\hline Michel, C.T. & 300 \\
\hline Mikolaskova, M. & 183,272 \\
\hline Milasinovic, D. & $91,280,281,289,301$ \\
\hline Milazzo, V. & 84 \\
\hline Millan Núñez-Cortés, J. & 48 \\
\hline Milwidsky, A. & 196 \\
\hline Min, J.Y. & 304 \\
\hline Mir, E. & 201 \\
\hline Mirocha, J. & 64,146 \\
\hline Mitchell, A. & 71 \\
\hline Mitsutake, R. & 332 \\
\hline Miyamoto, T. & 339 \\
\hline Miyazono, M. & 370 \\
\hline Mizuguchi, Y. & 355 \\
\hline Mmoudeh, A. & 106 \\
\hline Moeller, J.F. & 178 \\
\hline Moerland, P. & 101 \\
\hline Mogi, K. & 323 \\
\hline Mohamadi, A. & 360 \\
\hline Mohamed, H. & 260 \\
\hline Momin, A. & 212 \\
\hline Monaco, L. & 70 \\
\hline Monmeneu, J.V. & 270 \\
\hline Morales, C. & 207 \\
\hline Mori, T. & 166 \\
\hline Morís de la Tassa, C. & 199 \\
\hline Morís, C. & $177,232,249$ \\
\hline Morris, M. & 88 \\
\hline Moser, M. & 107 \\
\hline Mrkonjic, I. & 280 \\
\hline Muljadi, N. & 333 \\
\hline Müller, F.U. & 219 \\
\hline Munakata, M. & 299 \\
\hline Muñoz-García, A. & $232,249,177$ \\
\hline Muntean, D. & 235 \\
\hline Münzel, T. & 127 \\
\hline Nafafi, H. & 291 \\
\hline Nagasaka, R. & 365 \\
\hline Nakagawa, A. & 247,248 \\
\hline Nakagawa, K. & 264 \\
\hline Nakagawa, T. & 365 \\
\hline Nakajima, K. & 339 \\
\hline
\end{tabular}




\begin{tabular}{|c|c|}
\hline Nakajima, S. & 355 \\
\hline Nakamura, H. & 247,248 \\
\hline Nakashima, Y. & 264 \\
\hline Nam, J.H. & 231 \\
\hline Namdar, $\mathrm{H}$. & 290 \\
\hline Namdari, M. & 360 \\
\hline Nardi, P. & 208,322 \\
\hline Narvaez Muñoz, A. & 337,338 \\
\hline Nashef, S. & $206,327,340$ \\
\hline Navarra, T. & 367 \\
\hline Nayar, P. & 85 \\
\hline Nazari, A. & 360 \\
\hline Neele, A. & 101 \\
\hline Neto, M. & 30,302 \\
\hline Netuka, I. & 275 \\
\hline Neumann, J. & 219 \\
\hline Ngui, D. & 239 \\
\hline Nguyen, A. & 85 \\
\hline Nica, A.S. & 316,330 \\
\hline Nicolò, F. & 208 \\
\hline Nishizaka, M. & 370 \\
\hline Novakova, T. & 183,272 \\
\hline Novotny, $\mathrm{T}$. & 183 \\
\hline Obama, T. & 162 \\
\hline Obrebska, A. & 317 \\
\hline Ogawa, K. & 365 \\
\hline Okamura, K. & 332 \\
\hline Okura, H. & 334 \\
\hline Olevano, C. & 322 \\
\hline Olivares, C. & 226 \\
\hline Omura, S. & 166 \\
\hline Ondrus, $\mathrm{T}$. & 272 \\
\hline Oommen, A. & 303 \\
\hline Ordoñez, M. & 294 \\
\hline Ortas, M.D.R. & 125 \\
\hline Oschepkova, E. & 347 \\
\hline Ostrovsky, Y. & 202,213 \\
\hline Ozcan, Y. & 85 \\
\hline Pablo, E. & 329 \\
\hline Paizis, G. & 102 \\
\hline Palma dos Reis, R. & 30,302 \\
\hline Palmieri, C. & 353 \\
\hline Paltineanu, B. & $316,330,362$ \\
\hline Panisello-Tafalla, A. & 258,277 \\
\hline
\end{tabular}




\begin{tabular}{|c|c|}
\hline Pańkiw, O. & 253 \\
\hline Paolo, R. & 90,270 \\
\hline Paradossi, U. & 345,353 \\
\hline Park, J.H. & 34 \\
\hline Park, J.S. & 231 \\
\hline Park, M.H. & 304 \\
\hline Park, S.J. & 265 \\
\hline Park, S.M. & 265 \\
\hline Parker, J. & 192 \\
\hline Parvu, M. & 305,354 \\
\hline Pascual, I. & $177,232,249$ \\
\hline Pavlovic, V. & $91,129,281,289,301$ \\
\hline Pawlicki, L. & 318 \\
\hline Pedrinelli, R. & 174 \\
\hline Pellegrino, $A$. & 208,322 \\
\hline Pena, C. & $316,330,362$ \\
\hline Pereira, A. & 30,302 \\
\hline Pérez, L. & 226,233 \\
\hline Perovic, V. & 250,346 \\
\hline Pfleger, J. & 223 \\
\hline Piacentini, L. & $84,368,369$ \\
\hline Picelli, A. & 72 \\
\hline Pinto-Sietsma, S.J. & 101 \\
\hline Pirk, J. & 244,275 \\
\hline Pleskova, A. & 272 \\
\hline Poloczek, M. & 272 \\
\hline Pompilio, G. & 163 \\
\hline Pons, $\mathrm{F}$. & 271 \\
\hline Potluri, R. & 87,358 \\
\hline Potthoff, M. & 226 \\
\hline pourgholi, L. & 256 \\
\hline Pourmoghaddas, A. & 201 \\
\hline Poyet, R. & 271 \\
\hline Prat, S. & 181 \\
\hline Prior, S. & 243 \\
\hline Procida-Cervera, G. & 258,277 \\
\hline Provost, B. & 209 \\
\hline Pucci, A. & 288 \\
\hline Pul-Sang, S. & 335 \\
\hline Purnomowati, A. & 203 \\
\hline Qiu, M. & 239 \\
\hline Quah, T.J. & 310 \\
\hline Quarti, C. & 163 \\
\hline Queralt-Tomas, L. & 277 \\
\hline
\end{tabular}




\begin{tabular}{|c|c|}
\hline Queralt-Tomas, M.L. & 258 \\
\hline Radić, R. & 241 \\
\hline Ramboyong, R. & 278,319 \\
\hline Ramirez, M.F.L. & 333 \\
\hline Ramzy, D. & 64 \\
\hline Rankin, W. & 32 \\
\hline Raoufi, A. & 360 \\
\hline Rasoulian, B. & 360 \\
\hline Ravani, M. & 288 \\
\hline Rawas, H. & 128 \\
\hline Raya-Ampil, E. & 333 \\
\hline Rebello, E. & 285 \\
\hline Reija López, L. & $337,338,325$ \\
\hline Reims, H.M. & 103 \\
\hline Ren, J. & 185,341 \\
\hline Revnic, C.R. & $316,330,362$ \\
\hline Revnic, F. & $316,330,362$ \\
\hline Reynolds, A. & 180 \\
\hline Rezus, E. & 305 \\
\hline Ripolles-Vicente, R. & 258,277 \\
\hline Ristic, A. & 129,289 \\
\hline Ritchie, R. & 58 \\
\hline Rizzo, M. & 70,191 \\
\hline Robinson, S. & 243 \\
\hline Rodrigues, R. & 30,302 \\
\hline Rodriguez-Bermejo, A. & 350 \\
\hline Rodriguez, S. & 274 \\
\hline Rogelj, B. & 306 \\
\hline Rossetti, A. & 72 \\
\hline Rostiati, D. & 203 \\
\hline Ruan, L. & 357 \\
\hline Ruda, M. & 283,284 \\
\hline Ruedlinger, J. & 226,233 \\
\hline Ruggiero, N. & 88 \\
\hline Ruifang, Z. & 292 \\
\hline Ruiz-Garcia, J. & $167,308,309$ \\
\hline Ruiz-Nodar, J.M. & 199 \\
\hline Russkih, I. & 202 \\
\hline Ruvolo, G. & 208,322 \\
\hline Saadeh, C. & 200 \\
\hline Saavedra, N. & $226,233,245$ \\
\hline Sabaka, P. & 312,311 \\
\hline Sabas, M. & 319 \\
\hline Sabate, M. & 181 \\
\hline
\end{tabular}


Sabbah, H.N.

Sagic, D.

Saito, K.

Sakai, Y.

Sakamoto, T.

Sakurai, M.

Salarifar, M.

Salazar, L.

Salazar, L.A.

Samantha, A.

San Martin, M.Á.

Sanches Felix, A.C.

Sanchez Insa, E.

Sánchez Rubio, J.

Sánchez-Rodríguez, C.

Sanchez, R.

Sangiorgio, $\mathrm{P}$.

Santos, J.R.

Saporiti, F.

Sapp, R.

Sastradimaja, S.

Satoh, S.

Savage, M.P.

Sawatari, $\mathrm{H}$.

Sbrana, S.

Scafuri, A.

Scalise, F.

Schmitz, W.

Schuler, G.

Scott, A.

Segev, A.

Sekhavati, E.

Selthofer-Relatic, K.

Seo, G.H.

Seol, S.H.

Separham, A.

Sergio, G.B.

Sezavar, $\mathrm{H}$.

Sgorbini, L.

Shafiee, A.

Shahar, S.

Shalev, V.

Sharon, $\mathrm{O}$.

Shaw, R.
59

250,346

251

247, 248

370

323

$73,182,255$

226

233, 245

81

167, 294, 308, 309

240, 242

125

125

167, 294

102

9

230

368,369

243

203

166

14,88

370

288, 353

208, 322

33

219

40

356

42

73

241

336

335

290, 291

270

201

72

$73,182,255$

65

196

196

238 
Sheikh, M.

Shemesh, J.

Shety, A.

Shi, R.

Shimizu, $\mathrm{H}$.

Shin, D.G.

Shiomi, M.

Shket, A.

Shoker, A.

Shpektor, A.

Shumavets, V.

Siera Quiroga, J.

Sierra Quiroga, J.

Silberman, S.

Silva, J.

Šimko, F.

Simoncikova, P.

Simoni, G.

Simoni, J.

Simoni, P.

Simpson, R.

Smith, B.

Sohrabi, B.

Son, J.W.

Song, G.

Song, R.

Sorokina, M.

Sousa, A.C.

Spaltro, G.

Spangenburg, E.

Speciale, G.

Spinar, J.

Stankovic, G.

Stefan, V.

Steiner, R.

Stevens, W.

Stinson, W.

Stone, G.

Stouffer, R.

Straino, $\mathrm{S}$.

Strom, J.

Strzelecki, A.

Su, M.J.

Suesada, $\mathrm{H}$.
255

144

212

185,341

266

231

365

213

107

53

202, 213

325

337, 338

65

207

311,312

227

178

178

178

71

356

290

231, 265

75, 292

263

313, 314

30, 302

163

243

70

272

91, 129, 280, 281, 289, 301

127

241

298

344

86

21

163

45

261

286

351 
Suh, S.

Sule, A.

Sun, $C$.

Sundararajan, V.

Swedberg, K.

Sweeney, M.

Tabbalat, R.

Taddei, A.

Taguchi, I.

Takahara, Y.

Takahashi, A.

Takenaka, K.

Taki, A.

Tamura, S.

Tan, Q.

Tanaka, K.

Tanaka, M.

Taniguchi, N.

Tauber, R.

Tavazzi, L.

Tchicaya, A.

Teijeiro-Mestre, R.

Temizhan, A.

Teng, R.

Tesic, M.

Thadani, U.

Theobald, K.

Thomas, R.

Thompson, A.

Thygesen, K.A.

Tiksnadi, B.

Tokunou, T.

Tola, M.

Tomic, R.

Ton, $\mathrm{T}$.

Torgovicky, R.

Tori, M.O.

Trabka-Zawicki, A.

Trento, A.

Triadi, A.

Trillo, R.

Trinidad, S.

Tsiakou, A.

Tsonis, G.
336

310

185,341

356

25

160

106

345,353

247,248

323

355

166

29

365

74

339

246

355

65

25

104, 197

167, 308, 309

252

364

91

188

128

71

344

89

203

370

252

102

298

161

365

361

64

203

177, 232, 249

294

254

349 


\begin{tabular}{|c|c|}
\hline Tsunoda, $\mathrm{T}$. & 365 \\
\hline Uchida, Y. & 251 \\
\hline Ünal, S. & 252 \\
\hline Uppal, H. & 87,358 \\
\hline Urata, H. & 332 \\
\hline Vagts, D. & 127 \\
\hline Valera, L. & 230 \\
\hline Valle, V. & 238 \\
\hline Vancura, V. & 222 \\
\hline VandeWater, E. & 243 \\
\hline Vasilieva, E. & 55 \\
\hline vd Bossche, J. & 101 \\
\hline Vega, M. & $167,308,309$ \\
\hline Veloso Dutra, S.G. & 240,242 \\
\hline Ventura, C. & 97 \\
\hline Vered, Z. & 148 \\
\hline Vogrin, S. & 356 \\
\hline Voiculescu, D. & 315 \\
\hline Voinea, S. & $316,330,362$ \\
\hline von Korn, $\mathrm{H}$. & 127 \\
\hline von Segesser, K.L. & 210 \\
\hline Vrbska, J. & 275 \\
\hline Vucinic, S. & 250,346 \\
\hline Vukcevic, V. & $91,129,280,281,289,301$ \\
\hline Vukelič, K. & 306 \\
\hline Vymetalova, J. & 222 \\
\hline Wagner, D. & 104 \\
\hline Walinsky, P. & 88 \\
\hline Wang, L. & 328 \\
\hline Wang, $\mathrm{X}$. & 357 \\
\hline Wang, Y. & 185,341 \\
\hline Wang, Y.-G. & 80 \\
\hline Weitzman, D. & 196 \\
\hline Wesson, D.E. & 178 \\
\hline White, H.D. & 89 \\
\hline Wieczorek, E. & 262 \\
\hline Wilensky, R. & 24 \\
\hline Wong, N.C.W. & 160 \\
\hline Woodman, O. & 49 \\
\hline Xiaofen, W. & 357 \\
\hline Xie, Y. & 307 \\
\hline$X u, R$. & 357 \\
\hline Xuan, Y.-T. & 81 \\
\hline Yada, R. & 166 \\
\hline
\end{tabular}




\begin{tabular}{|c|c|}
\hline Yamada, T. & 355 \\
\hline Yamaguchi, T. & 162 \\
\hline Yamashiro, S. & 324,326 \\
\hline Yan, D. & 328 \\
\hline Yan, Y. & 341 \\
\hline Yao, Q. & 263 \\
\hline Yarmolovsky, A. & 198 \\
\hline Yates, P. & 128 \\
\hline Ye, Y. & 134 \\
\hline Yong, J. & 356 \\
\hline Yoshida, K. & 251 \\
\hline Yoshikazu, Y. & 246 \\
\hline Yoshida, S. & 371 \\
\hline Yoshino, G. & 267 \\
\hline Yoshitake, S. & 339 \\
\hline$Y u, C$. & 328 \\
\hline Yu, C.Q. & 366 \\
\hline Yu, F. & 85 \\
\hline Yu, Y. & 365 \\
\hline Zaman, M. & 87,358 \\
\hline Zarei, M. & 293 \\
\hline Zarifis, I. & 254 \\
\hline Zarrouk, A. & 260 \\
\hline Zbierska, K. & 253 \\
\hline Zdrojewski, Z. & 261 \\
\hline Zein, N. & 168 \\
\hline Zelias, A. & 361 \\
\hline Zhang, C. & 357 \\
\hline Zhang, R. & 75 \\
\hline Zhang, Y. & 185 \\
\hline Zheng, J. & 145 \\
\hline Zhou, Y. & 124 \\
\hline Ziaee, S. & $234,256,257$ \\
\hline Zivkovic, I. & 281 \\
\hline Zivkovic, M. & 280,301 \\
\hline Zmudka, K. & 361 \\
\hline Zuba-Surma, E. & 81 \\
\hline
\end{tabular}

